University of Tennessee Health Science Center

UTHSC Digital Commons

\title{
Systems Biology Approach to Identifying Host Interactive Pathways Modulating the Severity of Streptococcal Sepsis
}

\author{
Nourtan Fatthy Abdeltawab \\ University of Tennessee Health Science Center
}

Follow this and additional works at: https://dc.uthsc.edu/dissertations

Part of the Biological Phenomena, Cell Phenomena, and Immunity Commons, Genetic Processes Commons, and the Medical Genetics Commons

\section{Recommended Citation}

Abdeltawab, Nourtan Fatthy, "Systems Biology Approach to Identifying Host Interactive Pathways Modulating the Severity of Streptococcal Sepsis" (2010). Theses and Dissertations (ETD). Paper 5. http://dx.doi.org/10.21007/etd.cghs.2010.0003. 


\title{
Systems Biology Approach to Identifying Host Interactive Pathways Modulating the Severity of Streptococcal Sepsis
}

\begin{abstract}
Clinical outcomes of infectious diseases are controlled by complex interactions between the host and the pathogen. Epidemiological, genetic and molecular studies in my mentor's laboratory provided evidence that in invasive Group A streptococcal (GAS) infections, genetic variations in both bacteria and patients influenced the severity of GAS sepsis. Allelic variations in class II human leukocyte antigens (HLA) contributed significantly to differences in the severity of group A streptococcal sepsis caused by the same virulent strain of the bacteria. HLA class II molecules present streptococcal superantigens (SAgs) to $T$ cells, and variations in HLA class II molecules can strongly influence SAg responses. However, the bacteria produce a very large number of additional virulence factors that participate in the pathogenesis of this complex disease, and it is likely that host genes besides HLA class II molecules are also participating in modulating the severity of GAS sepsis. The main focus of this Ph.D. project was to identify additional host genes and pathways that may be modulating the severity of GAS sepsis.
\end{abstract}

To achieve this goal I applied a systems genetics approach, involving genome wide association studies (GWAS) of GAS sepsis in the Advanced Recombinant Inbred (ARI) panel of BXD mouse strains. We used this panel of ARI-BXD strains as a genetically diverse reference population to study differential severity of GAS sepsis as ARI-BXD strains diversity mimics the genetic diversity of human population. We assessed several traits associated with differential host responses to GAS sepsis, and analyzed variations in these traits in the context of mice genotypic variability, using genome-wide scans and the sophisticated analysis tools of WebQTL. This allowed us to map quantitative trait loci (QTL) associated with modulating susceptibility to severe GAS sepsis on chromosome (Chr) 2 and Chr X. The mapped QTLs strongly predicted disease severity (accounting for $25-30 \%$ of variance), and harbored highly polymorphic genes known to play important roles in innate immune responses. Based on linkage analyses, gene ontology, cocitation networks, and variations in gene expression, we identified interleukin 1 (IL1) and prostaglandin $E$ (PGE) pathways as prime candidates associated with modulating the severity of GAS sepsis.

To further investigate mechanisms underlying differential host susceptibility, we analyzed genome-wide differential gene expression in blood and spleens of uninfected vs. infected mice belonging to highly resistant or susceptible BXD strains, at selected times post infection. Our transcriptional analyses revealed common pathways between susceptible and resistant strains associated with innate immune response, e.g. Interferon signaling pathway.

Since our data has pointed to a strong association of differential response to GAS with innate immune responses, we explored if differences in the numbers of relevant immune cells among the BXD strains played a role in their differential susceptibility to GAS. We found no significant differences in numbers or percentages of immune cell populations between susceptible and resistant strains under normal, uninfected conditions. However, depletion of neutrophils and/or macrophages significantly increased the severity of GAS sepsis in both resistant and susceptible strains. Taken together, our data suggested that differences in mobilization and /or function of these cells between susceptible and resistant strains might play a role in modulating differential severity of GAS sepsis.

In conclusion, we found that variations in the severity of GAS sepsis have a strong genetic component that is complex and multigenic. Different combinations of genetic variants influenced theonset, progression, and severity of GAS sepsis and disease and ultimate outcome. Our overall approach of systems genetics, where we systematically dissected genetic, molecular, cellular and functional differences that may be associated with differential host susceptibility to GAS provided us with tremendous insight into disease mechanism. The knowledge gained can help the development of better 
diagnostics and means to predict disease severity based on a set of genetic and prognostic biomarkers to help customize patient care, to apply effective and more targeted therapeutic interventions and improve disease outcomes in septic patients.

\section{Document Type}

Dissertation

Degree Name

Doctor of Philosophy (Medical Science)

\section{Program}

Biological Science

\section{Research Advisor}

Malak Y. S. Kotb, Ph.D.

\section{Keywords}

Advanced Recombinant Inbred (ARI) BXD Mice Genome-wide Association Studies Immunogenetics of Infectious Diseases QTL Mapping Streptococcus pyogenes (GAS) Sepsis Systems Genetics

\section{Subject Categories}

Biological Phenomena, Cell Phenomena, and Immunity | Genetic Processes | Medical Genetics | Medical Sciences | Medicine and Health Sciences

\section{Comments}

Two year embargo expired May 2012. 
SYSTEMS BIOLOGY APPROACH TO IDENTIFYING HOST INTERACTIVE PATHWAYS MODULATING THE SEVERITY OF STREPTOCOCCAL SEPSIS

\author{
A Dissertation \\ Presented for \\ The Graduate Studies Council \\ The University of Tennessee \\ Health Science Center

\begin{abstract}
In Partial Fulfillment
Of the Requirements for the Degree

Doctor of Philosophy

From The University of Tennessee
\end{abstract}

By

Nourtan Fatthy Abdeltawab

May 2010 
Chapter 2 (c) 2007 by the Nature Publishing Group.

Chapter 3 (C) 2008 by Public Library of Science.

All other materials (C) 2010 by Nourtan Fatthy Abdeltawab.

All rights reserved. 


\section{DEDICATION}

To all my teachers, to whom I am forever indebted to, starting with my parents, and to my dissertation readers, I wish you would enjoy it as much as I did. 


\section{ACKNOWLEDGMENTS}

I am grateful to everyone who helped me in the process of achieving my Ph.D. degree. To start with, I would like to express my deep gratitude to Dr. Malak Kotb, who is not only my mentor; but also a dear friend who I am privileged to have. I have enjoyed my journey in her laboratory with all its ups and downs. I cannot thank her enough for her heartfelt dedication as a mentor that was fundamental to my scientific development and maturation. I am forever grateful for her everlasting support; she has taught me priceless lessons in my scientific and social life.

I would like to thank my committee members; each and every member has been crucial towards my scientific development. Their critiques and suggestions towards better dissecting the mechanisms of the studied sepsis model have been tremendously constructive. I would like to especially thank Dr. Robert Williams and his research team with whom I enjoyed working closely as a collaborating student throughout my Ph.D. research project.

I would like to extend a special thanks to Dr. Kotb's research team, present and previous members, as with the extensive animal experiments done in this dissertation, I could not have ever done it on my own. While working with Dr. Kotb laboratory members, I have learned something valuable from each and every one of them. I would like to thank all my co-authors on my publications. I would like to extend a special thanks to Dr. William Taylor for sharing his experience with me during my gene expression analysis studies. I am forever grateful to Dr. Yan Jiao for our various collaboration studies that will result in multiple manuscripts to be soon published.

On a personal level, I would like to thank all my friends along this journey. There are too many to mention everyone, they all have been very supportive. I would like to especially thank Mrs. Sarah Rowe Hasty, who has been a great asset to my success; she is a living example of sincerity and devotion. She is a genuinely dedicated scientist that I enjoyed learning from and working with. I will always cherish the time we spent together whether in the lab or outside it, as she has been a wonderful second mother to me.

Words cannot express enough my deep gratefulness to my parents, my father, Prof. Dr. Fatthy M. Abdeltawab and my mother, Prof. Dr. Maie F. Ali. They have been tremendously supportive throughout my Ph.D. journey. Last but not least, I would like to thank my brother, Amr and his beautiful wife Samah, and their cute daughter Rose, who lightens up my days with her cute playful giggles.

Finally, I am grateful to various funding agencies that supported this work. These agencies include Medical Research Service, Department of Veterans Affairs (Merit Award to M.K.), the U.S. Army Medical Research Acquisition Activity (W81XWH-051-0227 to M.K), AI40198-06 grant from NIH, National Institute of Allergy and Infectious Diseases (NIAID, to M.K.). Development and maintenance of Gene Network and the BXD Colony is partly supported by INIA and Human Brain Project funded 
jointly by the NIMH, NIDA, and NIAAA (P20-DA 21131, U01AA13499 to R.W.W.), NCI MMHCC (U01CA105417 to R.W.W.), and the Biomedical Informatics Research Network (BIRN), NCRR (U24 RR021760 to R.W.W.). Parts of this work were awarded ASM Student travel award, funded by Richard and Mary Finkelstein grants (ASM award to N.F.A.). 


\begin{abstract}
Clinical outcomes of infectious diseases are controlled by complex interactions between the host and the pathogen. Epidemiological, genetic and molecular studies in my mentor's laboratory provided evidence that in invasive Group A streptococcal (GAS) infections, genetic variations in both bacteria and patients influenced the severity of GAS sepsis. Allelic variations in class II human leukocyte antigens (HLA) contributed significantly to differences in the severity of group A streptococcal sepsis caused by the same virulent strain of the bacteria. HLA class II molecules present streptococcal superantigens (SAgs) to T cells, and variations in HLA class II molecules can strongly influence SAg responses. However, the bacteria produce a very large number of additional virulence factors that participate in the pathogenesis of this complex disease, and it is likely that host genes besides HLA class II molecules are also participating in modulating the severity of GAS sepsis. The main focus of this Ph.D. project was to identify additional host genes and pathways that may be modulating the severity of GAS sepsis.
\end{abstract}

To achieve this goal I applied a systems genetics approach, involving genome wide association studies (GWAS) of GAS sepsis in the Advanced Recombinant Inbred (ARI) panel of BXD mouse strains. We used this panel of ARI-BXD strains as a genetically diverse reference population to study differential severity of GAS sepsis as ARI-BXD strains diversity mimics the genetic diversity of human population. We assessed several traits associated with differential host responses to GAS sepsis, and analyzed variations in these traits in the context of mice genotypic variability, using genome-wide scans and the sophisticated analysis tools of WebQTL. This allowed us to map quantitative trait loci (QTL) associated with modulating susceptibility to severe GAS sepsis on chromosome (Chr) 2 and $\mathrm{Chr} \mathrm{X}$. The mapped QTLs strongly predicted disease severity (accounting for $25-30 \%$ of variance), and harbored highly polymorphic genes known to play important roles in innate immune responses. Based on linkage analyses, gene ontology, co-citation networks, and variations in gene expression, we identified interleukin 1 (IL1) and prostaglandin E (PGE) pathways as prime candidates associated with modulating the severity of GAS sepsis.

To further investigate mechanisms underlying differential host susceptibility, we analyzed genome-wide differential gene expression in blood and spleens of uninfected vs. infected mice belonging to highly resistant or susceptible BXD strains, at selected times post infection. Our transcriptional analyses revealed common pathways between susceptible and resistant strains associated with innate immune response, e.g. Interferon signaling pathway.

Since our data has pointed to a strong association of differential response to GAS with innate immune responses, we explored if differences in the numbers of relevant immune cells among the BXD strains played a role in their differential susceptibility to GAS. We found no significant differences in numbers or percentages of immune cell populations between susceptible and resistant strains under normal, uninfected 
conditions. However, depletion of neutrophils and/or macrophages significantly increased the severity of GAS sepsis in both resistant and susceptible strains. Taken together, our data suggested that differences in mobilization and/or function of these cells between susceptible and resistant strains might play a role in modulating differential severity of GAS sepsis.

In conclusion, we found that variations in the severity of GAS sepsis have a strong genetic component that is complex and multigenic. Different combinations of genetic variants influenced the onset, progression, and severity of GAS sepsis and disease and ultimate outcome. Our overall approach of systems genetics, where we systematically dissected genetic, molecular, cellular and functional differences that may be associated with differential host susceptibility to GAS provided us with tremendous insight into disease mechanism. The knowledge gained can help the development of better diagnostics and means to predict disease severity based on a set of genetic and prognostic biomarkers to help customize patient care, to apply effective and more targeted therapeutic interventions and improve disease outcomes in septic patients. 


\section{TABLE OF CONTENTS}

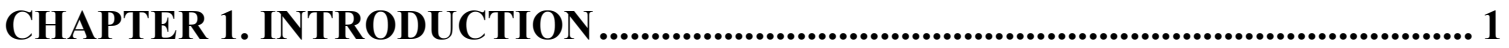

Pathogenesis Mechanisms of Streptococcus pyogenes ............................................... 1

Streptococcal Arsenal of Virulence Factors ............................................................... 1

Streptococcal Sepsis as a Model of Host-pathogen Interactions .................................... 4

Infectious Diseases as Complex Traits that Require a Systems Approach to Better

Understand Them............................................................................................ 5

Systems Genetics Approach - Definition and Tools .................................................. 6

Overview of Aims, Results, and Organization of the Dissertation................................ 9

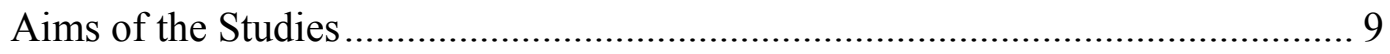

Results and Organization of the Dissertation...................................................... 10

\section{CHAPTER 2. SUSCEPTIBILITY TO SEVERE STREPTOCOCCAL SEPSIS: USE OF A LARGE SET OF ISOGENIC MOUSE LINES TO STUDY GENETIC AND ENVIRONMENTAL FACTORS ............................................ 12}

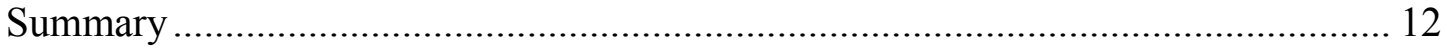

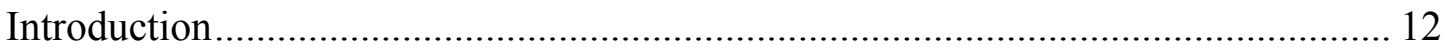

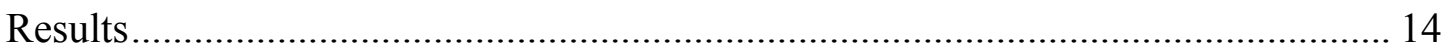

Survival Scores Show Differential Susceptibility to Severe GAS Sepsis ............ 14

Bacterial Loads in Blood and Organs Show Various Degrees of Bacteremia

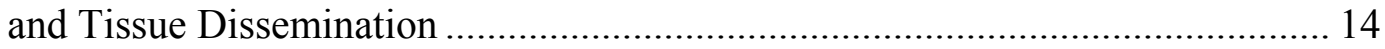

Statistical Analysis Evaluates the Confounding Factors Affecting Mice

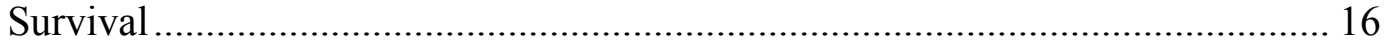

Analysis of Covariates Underscores the Role of Genetic Background in Determining Animal Susceptibility to GAS ...................................................... 18

BXD Strains Exhibited Phenotype Variability Outside the Range of the

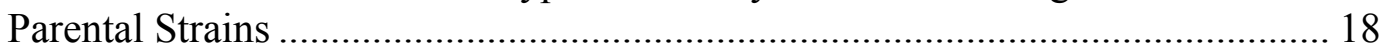

Controlled Time-course Experiments Show the Kinetics of Bacterial Spread and Confirm Results of Population-based Experiments ......................................... 22

Preliminary Quantitative Trait Loci Mapping ………….................................... 22

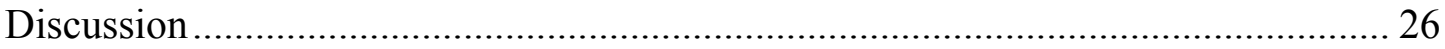

The Strength of the BXD Recombinant Inbred Population Model....................... 26

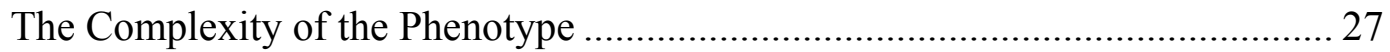

The Power of Statistical Analysis .................................................................... 28

The Preliminary Quantitative Trait Loci Mapping Results ................................... 28

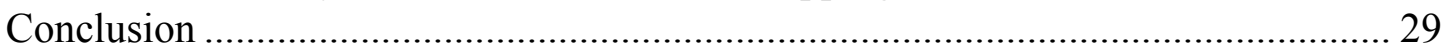

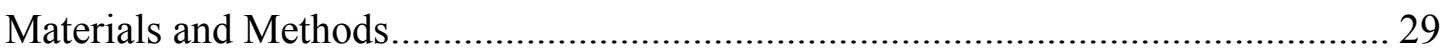

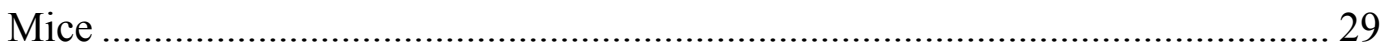

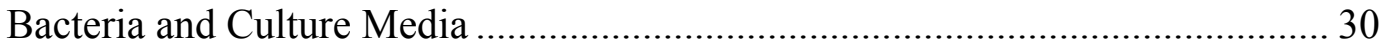

Experimental Design and Infection Scheme ..................................................... 30

Design of Controlled Time-course Experiments ................................................ 30 


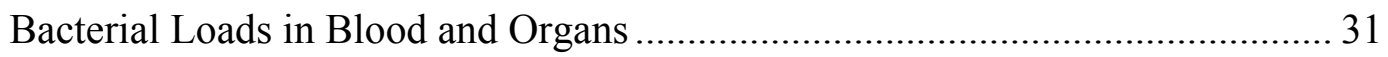

Data Handling and Statistical Analysis............................................................ 31

Preliminary Quantitative Trait Loci Mapping ................................................... 31

\section{CHAPTER 3. AN UNBIASED SYSTEMS GENETICS APPROACH TO MAPPING GENETIC LOCI MODULATING SUSCEPTIBILITY TO SEVERE STREPTOCOCCAL SEPSIS ............................................................... 32}

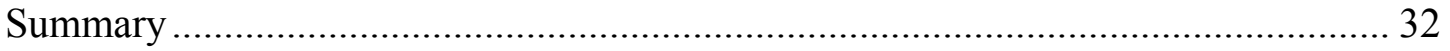

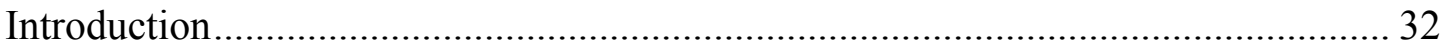

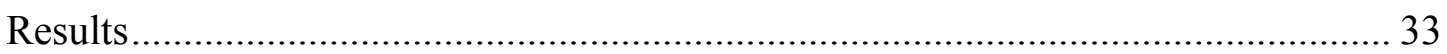

Variable Susceptibility to Severe GAS Sepsis in Genetically Distinct Mice ....... 33

Genome-wide Scans for Mapping GAS Susceptibility Quantitative Trait

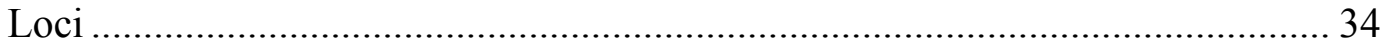

In silico Prediction of BXD Susceptibility to Severe GAS Sepsis ....................... 40

Mining for Candidate Genes and Pathways in Mapped Loci ................................. 44

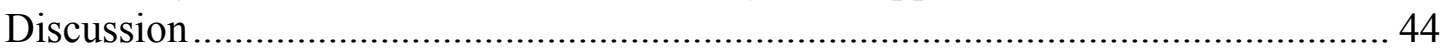

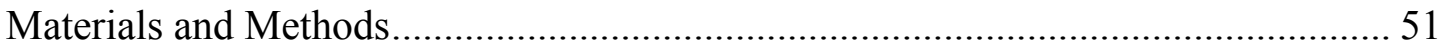

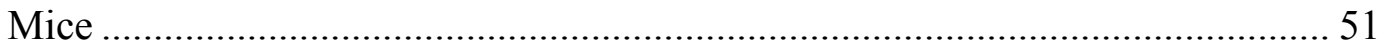

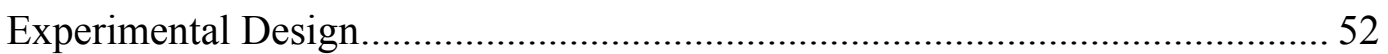

Population-based experiments ……………………….......................... 52

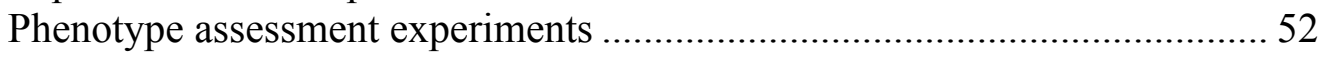

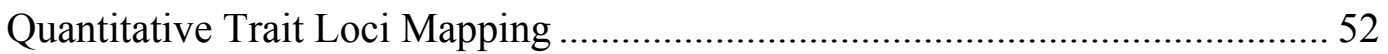

Quantitative PCR Analysis for Target Genes Expression .................................... 53

Identification of Differentially Expressed Genes in the Mapped Interval and Bioinformatics Functional Pathways Analyses ................................................. 53

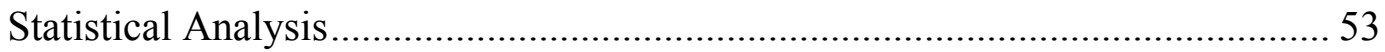

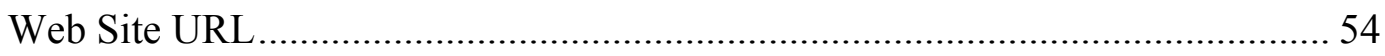

\section{CHAPTER 4. IDENTIFICATION OF SOLUBLE AND GENETIC} BIOMARKERS ASSOCIATED WITH DIFFERENT OUTCOMES OF GAS SEPSIS REVEALS UNDERLYING MECHANISMS OF DIFFERENTIAL

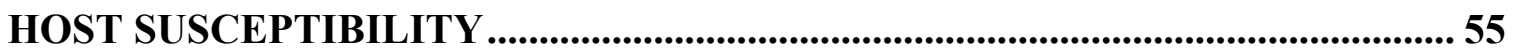

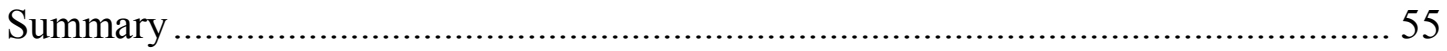

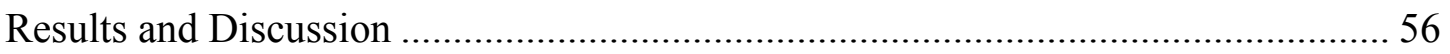

Comparison of Genome-wide Differential Expression in Blood between Susceptible and Resistant Strains at Selected Times Post Infection...................... 56

Soluble Biomarkers Associated with Differential Susceptibility in Select Susceptible and Resistant Strains at Multiple Time Series Post-infection ........... 72

Analysis of the Role of Prostaglandin at Early Stage of Infection ........................ 86

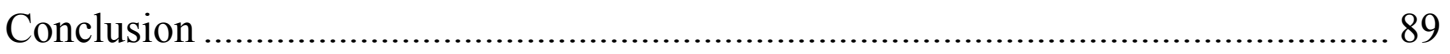

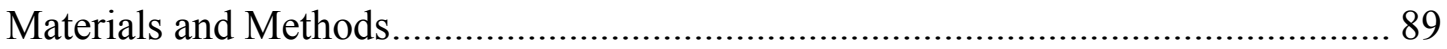

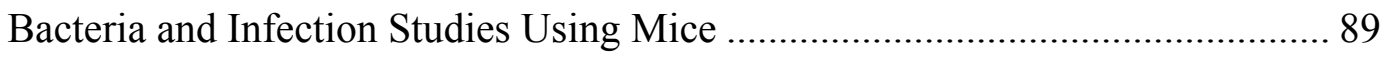

Experimental Design of Time Series Infection Studies .......................................... 89

Processing of Blood and Spleen Samples and Purification of RNA .................... 90 
Microarray Platform and Design .................................................................... 90

Microarray Data Processing and Analysis ......................................................... 91

Identification of Differentially Expressed Genes and Functional Pathways

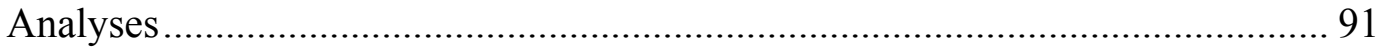

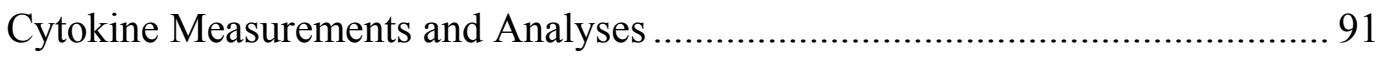

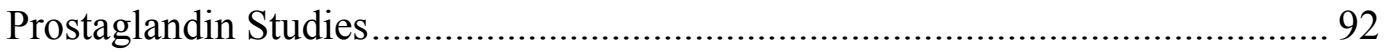

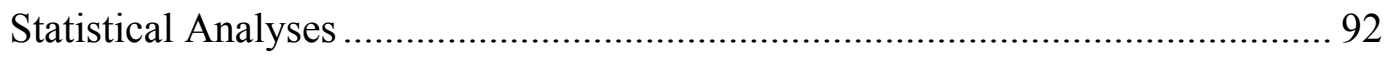

\section{CHAPTER 5. ANALYSIS OF CELLULAR POPULATIONS MODULATING} DIFFERENTIAL RESPONSE TO SEVERE GAS SEPSIS.....................................94

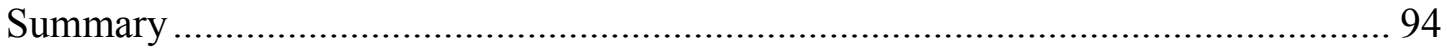

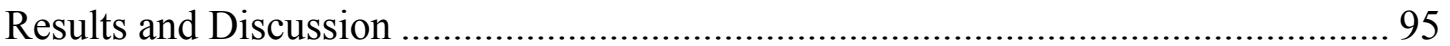

Profiling of Blood Cell Populations across Selected Resistant and Susceptible

Strains Reveals Possible Migratory and Functional Differences Rather than Cellular Availability as a Possible Mechanism of Individual Variations in Susceptibility to Severe GAS Sepsis

Immunophenotyping Analyses of Cellular Population under Normal

Physiological Conditions in Spleen of BXD Strains Revealed No Differences

in Cellular Numbers or Relative Percentages among Susceptible and

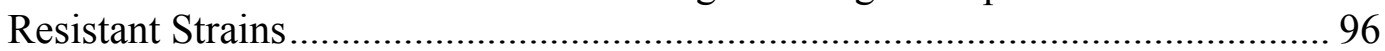

In vivo Manipulation of Cellular Populations in Susceptible and Resistant

Strains: Depletion of Gr- $1^{+}$Cells and Macrophages Rendered Resistant

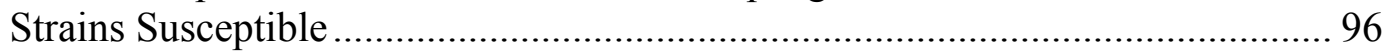

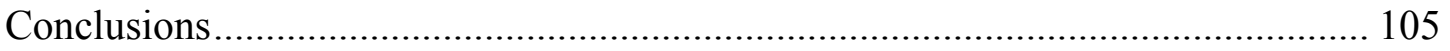

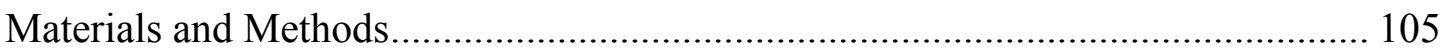

Experimental Design of Immunophenotyping Studies ....................................... 105

Differential Blood Counts............................................................................ 107

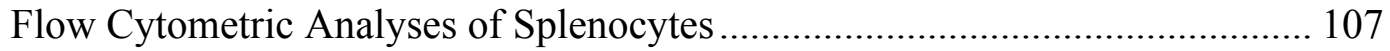

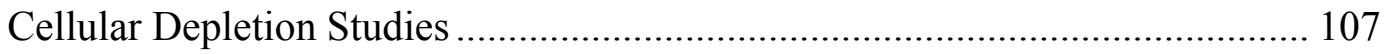

Data Analysis and Statistical Analyses ............................................................... 108

CHAPTER 6. SUMMARY FUTURE DIRECTIONS AND CONCLUSIONS....... 109

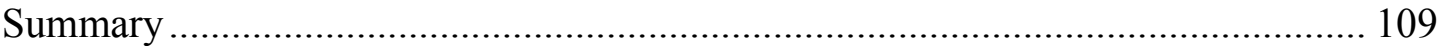

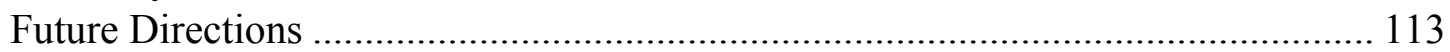

Dissection of Migratory and Functional Differences of Immune Cell

Populations between Susceptible and Resistant Strains ..................................... 113

Analysis of Relationship of Prostaglandins and Modulation of Differential

Response to GAS ....................................................................................... 114

Cytokine Profiling Associated with Susceptibility to GAS Sepsis..................... 116

Analysis of the Role of Nod-like Receptors as a Candidate Pattern

Recognition Receptor Associated with Modulation of Differential Response

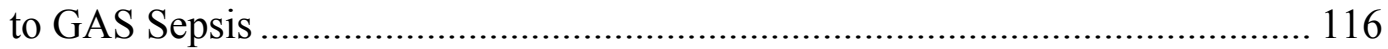

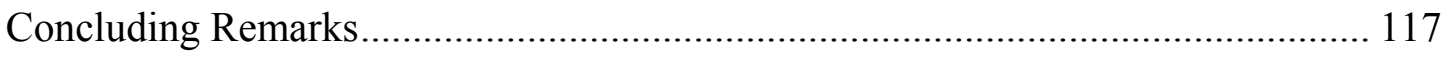

LIST OF REFERENCES ............................................................................................. 118 
APPENDIX A. PRIMER SEQUENCES USED FOR QPCR AND THE

RELATIVE EXPRESSION LEVELS OF CANDIDATE GENES.

APPENDIX B. LIST OF GENES AND TRANSCRIPTS IN THE MAPPED

LOCI

APPENDIX C. POLYMORPHISM ANALYSIS OF GENES AND

TRANSCRIPTS IN THE MAPPED LOCI

APPENDIX D. LIST OF PUBLISHED LOCI AND GENES ON

CHROMOSOME 2 THAT ARE ASSOCIATED WITH DIFFERENTIAL

SUSCEPTIBILITY TO INFECTIOUS DISEASES

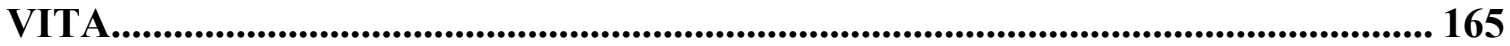




\section{LIST OF TABLES}

Table 2-1. Mouse strains used in the final analysis of this study ............................. 19

Table 2-2. Analysis of Variance for Survival Index............................................... 19

Table 2-3. Extent of invasiveness in time-course experiments, as measured by bacterial loads in spleen, liver, and lung .......................................... 25

Table 3-1. The relative expression levels of candidate genes post infection in resistant and susceptible strains expressed as ratio of post/pre infection in susceptible and resistant strains

Table 4-1. Differentially expressed genes in peripheral blood of resistant and susceptible strains at $8 \mathrm{hrs}$ post GAS infection

Table 4-2. Differentially expressed genes in peripheral blood of resistant and susceptible strains at $12 \mathrm{hrs}$ post GAS infection.

Table 5-1. Cell surface markers that were used to defines spleen immune cells populations characterized in the current study....

Table A-1. Primer sequences used in quantitative PCR assays for candidate genes

Table A-2. Relative expression levels of candidate gene list expressed as mean fold difference between pre- and post-infection \pm standard deviation (SD) in selected resistant and susceptible strains.

Table B-1. Genes and their SNPs in mapped locus on Chr 2 between 22-34 Mb .... 142

Table B-2. Genes and their SNPs in mapped locus on Chr 2 between $124-150 \mathrm{Mb}$

Table B-3. Genes and their SNPs in mapped locus on Chr X between 50-100Mb ... 150

Table C-1. SNP analysis of genes in mapped locus on Chr 2 between $22-34 \mathrm{Mb}$...... 156

Table C-2. SNP analysis of genes in mapped locus on Chr 2 between $125-150 \mathrm{Mb}$.

Table C-3. SNP analysis of genes in mapped locus on Chr X between 50-100Mb... 160

Table D-1. Diseases and mouse models associated with quantitative trait loci and genes located at Chromosome 2 loci mapped in current study.... 164 


\section{LIST OF FIGURES}

Figure 1-1. An overview of group A streptococcal main virulence factors................... 2

Figure 1-2. Breeding scheme used for generation of RI strains of mice ...................... 7

Figure 2-1. Distribution and correlation of survival scores and bacteremia indices.... 15

Figure 2-2. Effect of non-genetic factors on the survival of BXD mice and their parental strains ............................................................................ 17

Figure 2-3. Differential susceptibility of BXD strains to invasive GAS .................... 20

Figure 2-4. Variation in bacteremia and spleen dissemination in different BXD

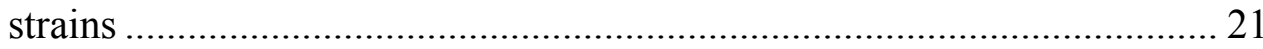

Figure 2-5. Kinetics of bacterial dissemination in lung, liver, and spleen of six BXD strains

Figure 3-1. Differential susceptibility to GAS sepsis among different BXD strains

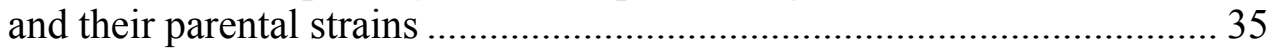

Figure 3-2. Genome-wide scan for mice susceptibility to GAS sepsis showing mapped QTL on Chr 2 .............................................................. 38

Figure 3-3. Recombinant inbred BXD strain distribution patterns at region of interest on $\mathrm{Chr} 2$.................................................................... 41

Figure 3-4. Patterns of differential gene expression levels of candidate genes post infection in susceptible and resistant strains ............................................. 46

Figure 3-5. Functional network of genes modulating GAS QTL ................................ 47

Figure 4-1. Experimental design to test gene expression differences associated with differential susceptibility to severe GAS sepsis at selected stages of infection.

Figure 4-2. Pathways associated with differentially expressed genes at 8 hours post infection in peripheral blood of resistant and susceptible strains compared to uninfected $(0 \mathrm{hr})$ mice of respective strains

Figure 4-3. Pathway analysis of differentially expressed genes at $12 \mathrm{hrs}$ post infection in peripheral blood of resistant and susceptible strains compared to uninfected $(0 \mathrm{hr})$ mice of respective strains

Figure 4-4. Pathways associated with differentially expressed genes in spleens of uninfected versus infected resistant and susceptible ARI-BXD strains

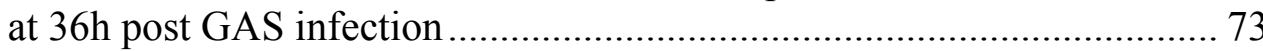

Figure 4-5. TNF $\alpha$ plasma levels profiles across early time points post infection comparing selected highly susceptible and highly resistant ARI-BXD strains

Figure 4-6. IL6 plasma levels profiles across early time points post infection comparing selected highly susceptible and highly resistant ARI-BXD strains 
Figure 4-7. IL10 plasma levels profiles across early time points post infection comparing selected highly susceptible and highly resistant ARI-BXD strains

Figure 4-8. IFN $\gamma$ plasma levels profiles across early time points post infection comparing selected highly susceptible and highly resistant ARI-BXD strains

Figure 4-9. Comparison of ratios of anti-inflammatory to pro-inflammatory cytokines, comparing ratios of medians of IL10/IFN $\gamma$ plasma levels across early time points post infection in selected highly susceptible and highly resistant ARI-BXD strains

Figure 4-10. Analysis of prostaglandin role at early stages of severe GAS sepsis........ 88

Figure 5-1 Differential blood count of selected susceptible and resistant BXD strains

Figure 5-2. Diagram showing a scheme for flow cytometric analysis of immune cell populations in spleen employed in the current study

Figure 5-3. Immunophenotyping of spleen cellular populations in selected resistant and susceptible BXD strains

Figure 5-4. Depletion of Gr-1+ cells in selected resistant and susceptible BXD strains shows reversal of survival in response to GAS sepsis.

Figure 5-5. Kinetics of neutrophils and monocytes at early time points post GAS infection.

Figure 5-6. Comparison of the kinetics of neutrophils and monocytes numbers in susceptible versus resistant strains during the first 2 hours post GAS infection 


\section{CHAPTER 1. INTRODUCTION}

\section{Pathogenesis Mechanisms of Streptococcus pyogenes}

Streptococcus pyogenes is an important human pathogen that can cause a wide spectrum of diseases with varying degrees of severity (reviewed in [Cunningham, 2000; Kotb et al., 2003]). These diseases range from mild sore throat or skin infections to severe and often deadly ones, such as streptococcal toxic shock syndrome (STSS) and necrotizing fasciitis (NF), known as flesh-eating disease, giving the bacteria its nickname as the flesh-eating bacteria. Certain individuals develop post-streptococcal nonsuppurative sequelae that include glomeronephritis, acute rheumatic fever, poststreptococcal arthritis, and neurological disorders collectively known as pediatric autoimmune neuropsychiatric disorders associated with streptococcal infections (PANDAS).

The wide range of diseases associated with Streptococcus pyogenes infections and their varying degrees of severity has intrigued us and other researchers to study the basis for the variable outcomes of the infection. Research groups have long debated over which is more vital towards the final outcome of streptococcal diseases, the bacteria or the host, with evidence supporting each side. Lately, it has become evident that interactions between host factors, which are influenced by their genetic background, and environmental factors, and the bacterial arsenal of virulence factors shape the final outcome of group A streptococcal (GAS) infection. I will briefly review the bacteria virulence factors and then elaborate on host genes associated with modulating the susceptibility to infectious diseases and sepsis.

\section{Streptococcal Arsenal of Virulence Factors}

Streptococcus pyogenes expresses multiple virulence factors that are tailored to their human host. Not all of these factors are expressed at the same time as Streptococcus pyogenes (also known as (GAS)) senses its environment and accordingly restructures its community to where mutants that are best fit for specific host environment are selected. These mutants have distinct genetic expression patterns. Examples of GAS virulence factors are shown in Figure 1-1. Some virulence factors are secreted and others are surface bound, of note, superantigens (SAgs), which are a large group of secreted proteins (exotoxins) and are associated with severe GAS sepsis and STSS. GAS produces virulence factors that specifically impair host mechanisms of bacterial killing; for example, GAS interferes with host signals for neutrophil chemotaxis, migration and function. Streptococcal chemokine protease A (ScpA), also known as the complement 5a (C5a) peptidase, degrades host C5a an important chemoattractant. Meanwhile, streptococcal chemokine protease $\mathrm{C}$ (ScpC), also known as interleukin 8 (IL8) degrading enzyme, degrades host IL8 thus impairing one of neutrophil chemotaxis factors (Sjolinder et al., 2008; Gleich-Theurer et al., 2009). Streptococcal inhibitor of complement (SIC) inhibits complement membrane attack complex and was also recently associated with inhibition of several of the human innate immune machinery namely, lysozyme, secretory 
Figure 1-1. An overview of group A streptococcal main virulence factors.

GAS displays multiple virulence factors depending on site and stage of infection, shown are selected virulence factors. Each of these virulence factors has been tailored to counteract one or more of the host defenses. For example, ScpA (streptococcal chemokine protease A, also known as C5a peptidase) degrades host C5a, and ScpC (streptococcal chemokine protease C) degrades interleukin 8 (IL8), with a net result of hindering the recruitment of neutrophils to site of infection (Hidalgo-Grass et al., 2006; Sjolinder et al., 2008; Zinkernagel et al., 2008). Streptococcal inhibitor of complement (SIC) as its name suggests, inhibits complement membrane attack complex and was also recently associated with inhibition of four more proteins of the human innate immune machinery namely, lysozyme, secretory leukocyte proteinase inhibitor, human $\alpha$-defensin 1 and LL-37 (Johansson et al., 2008; Minami et al., 2009). M protein binds and counteracts host's counter-regulatory factors e.g. host fibronectin. Streptokinase (Ska) degrades human plasminogen into plasmin thereby, dissolving blood clots. EndoS, endoglycosidase S; FBP54, fibronectin-binding protein 54; Mac, Mac1-like protein; MHC, major histocompatibility complex; PFBP, pyogenes fibronectin-binding protein; $\mathrm{Sfb}$, streptococcal fibronectin-binding protein; SlaA, streptococcal phospholipase A2; cytotoxins streptolysin O (SLO) and streptolysin S (SLS); Spe, streptococcal pyogenic exotoxin (aka superantigens (SAgs)); Smez, streptococcal mitogenic exotoxin Z; SSA, streptococcal superantigen A; GRAB, G-related a2-macroglobulin-binding protein; NADGH, Nicotine adenine dinucleotide (NAD) glycohydrolase; and TCR, T-cell receptor. Source: Adapted with permission. Mitchell, T.J. (2003) The pathogenesis of streptococcal infections: from tooth decay to meningitis. Nat Rev Microbiol 1: 219-230. 


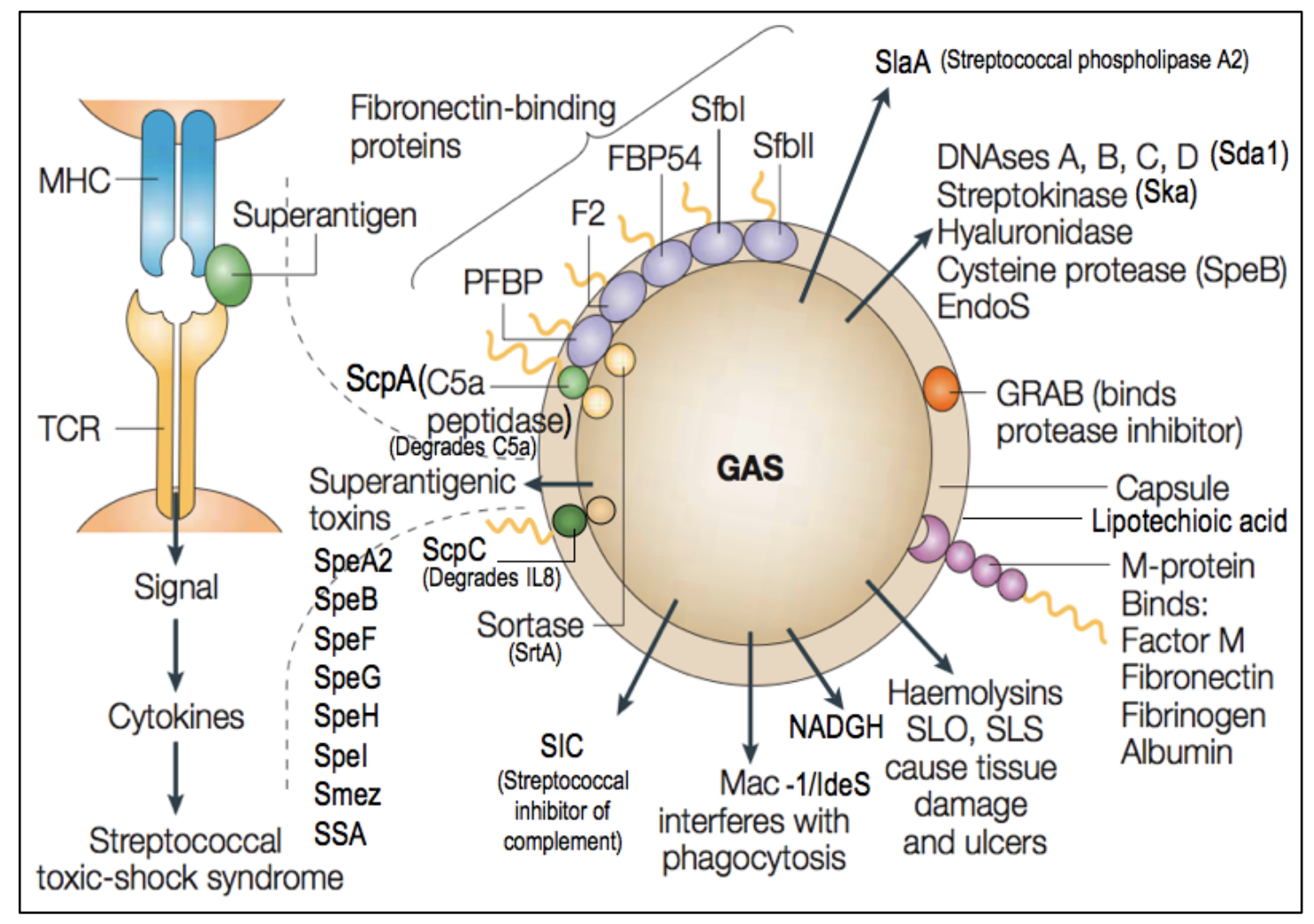


leukocyte proteinase inhibitor, human $\alpha$-defensin 1 and LL-37 (Johansson et al., 2008; Minami et al., 2009). Another example of GAS interfering with neutrophil killing mechanisms is Sda1, a potent DNase produced by M1T1 (GAS strain under study). Sda1 dissolves neutrophil extracellular traps (NETs), which are DNA filaments produced by neutrophils and contain histones and antimicrobial peptides, allowing the bacteria to escape the entrapment and on mechanism of killing by neutrophil (Aziz et al., 2004b; Buchanan et al., 2006; Walker et al., 2007).

According to site and stage of infection, GAS virulence factor genes are turned on or off to survive the host immune response and adapt to its environment. How the bacterium does that is not completely understood, but it is postulated that according to the environmental pressure, GAS selects for the most-fit mutants, sacrificing even the wild type. This has been shown in several recent studies by our group and others (Buchanan et al., 2006; Walker et al., 2007) and reviewed by (Aziz and Kotb, 2008; Bessen, 2009)

In the studies discussed in this dissertation, I used a hypervirulent clonal GAS strain, designated M1T1. This hypervirulent M1T1 clonal strain, along with M3, M18, and M28 strains, has been associated with the resurgence of the severe GAS sepsis in the 1980s (Johnson et al., 1992; Hoge et al., 1993). M1 and M3 GAS strains have displayed highest associations with severe GAS sepsis (STSS) and NF (Hauser et al., 1991; Johnson et al., 1992). Our research focus has been on the M1T1 strain, which has been consistently isolated over the past 20 years from patients with both noninvasive and invasive disease displaying varying degrees of severity (Chatellier et al., 2000; Johnson et al., 2002; Ikebe et al., 2007). The hypervirulence of this strain is believed to be due to the acquisition of virulence factors through horizontal gene transfer, recombination and mutations (reviewed in [Aziz and Kotb, 2008]).

\section{Streptococcal Sepsis as a Model of Host-pathogen Interactions}

In studies presented in this dissertation, our focus was to determine the basis for differential host susceptibility to one of the most severe GAS invasive diseases, streptococcal toxic shock syndrome (STSS). STSS is an invasive disease of GAS, where bacteria reach an otherwise sterile site in the body, e.g. blood, and the patient has hypotension, plus two or more of the following clinical symptoms that are indicative of multiple organ failure: renal impairment, coagulopathy, hepatic involvement or respiratory distress. A STSS case is classified as probable if GAS is isolated from a nonsterile site or fluid, e.g. throat, and the patient displays hypotension and two or more of the above-mentioned symptoms (Lappin and Ferguson, 2009).

The pathophysiology of STSS has multiple aspects and is associated with host interactions and response to the bacterial arsenal of virulence factors including superantigens (SAgs). The Kotb research group has found, through epidemiological studies, that the severity of GAS sepsis is associated with allelic variations in human leukocyte antigen (HLA) class II (Kotb et al., 2002). Patients with GAS sepsis expressing HLA class II (DR15/DQ6) haplotype were protected from severe GAS sepsis, whereas 
those with HLA class II (DR14/DQ5) haplotype were at high risk for developing severe and often fatal forms of the disease (Kotb et al., 2002; Norrby-Teglund et al., 2002; Nooh et al., 2007). Ensuing studies, in vitro and in vivo, dissected the molecular mechanisms underlying these differential responses and helped to dissect the role of GAS SAgs in severe GAS sepsis and their interactions with HLA-II (Kotb et al., 2002; Norrby-Teglund et al., 2002; Welcher et al., 2002; Kotb et al., 2003; Kotb, 2004; Nooh et al., 2007).

Whereas HLA-II allelic variations clearly influenced disease severity, it was clear that other host factors must also be participating in the pathogenesis of GAS sepsis because different inbred mice that do not express HLA-II were also found to display differential susceptibility to GAS sepsis (Medina et al., 2001).This observation was interesting because mice are normally less susceptible to GAS SAgs because they have an inherent poorer affinity of MHC-II to GAS SAgs. Therefore, other bacterial virulence factors, other than SAgs, and hence other host factors, other than HLA-II, are also likely to modulate differential severity to GAS sepsis. Moreover, the bacteria produce a number of additional, important virulence factors that must also participate in the pathogenesis of GAS sepsis. The overall aim of my study was to identify additional host factors modulating susceptibility to severe GAS sepsis and to identify host genes and pathways involved in this disease (Aziz et al., 2007; Abdeltawab et al., 2008).

\section{Infectious Diseases as Complex Traits that Require a Systems Approach to Better Understand Them}

It is now established that infectious diseases are a manifestation of complex traits modulated by the interaction of host factors and genetic background with the pathogen virulence factors and other environmental factors. Complex interactions between the pathogen virulence factors and host immune-related genetic makeup determine the progression and outcomes of the infection. Host genetic variations modulating differential response to infectious diseases have long been studied (Hornef et al., 2002; Fortin et al., 2007; Carvalho et al., 2009; Chai et al., 2009; Deghmane et al., 2009; Ko et al., 2009; Zhang et al., 2009a). Variations in host response can be attributed to variations in pathogen recognition, prior exposure and preexisting immunity, immune regulation and/or quantitative differences in the immune response mounted to the infectious agents (reviewed in [Frank, 2002]). Variation in host recognition of pathogens and their virulence components can arise from a number of factors, including variations in many immune genes, including genes encoding pattern recognition receptors (PRR), and major histocompatibility complex (MHC) molecules. Also, variations in genes involved in immune regulation can strongly affect the host response to the infection. An added level of host immune response variability arises from polymorphisms of key immune-related genes.

Two main approaches have been employed to identify host genetic polymorphisms associated with infectious diseases. The first approach is to examine a candidate gene or genes for polymorphisms and analyze the distribution of these polymorphisms in patients or infected subjects vs. a normal group. The hypotheses are usually based on plausible associations based on the mechanism of diseases studied and 
prior reports in the literature. The second approach employed is genome-wide association studies (GWAS). In GWAS no assumptions are made as to which gene might be involved and a scan of associations of phenotype with genotypes is performed. Such approaches have been widely applied in animal models and moving forward with using human subjects, as more projects like the human haplotype map (HapMap) (Frazer et al., 2007) and the Wellcome Trust Case Control Consortium (2007) are establishing genomic repositories for case-control studies of common diseases.

\section{Systems Genetics Approach - Definition and Tools}

Systems genetics is an approach that studies the effects of multiple genes on modulating disease phenotypes. Instead of the classical one gene, one disease approach, systems genetics attributes variations in phenotypes of a trait to a network of genes or loci that are in turn affected by environmental conditions (Sieberts and Schadt, 2007). Instead of looking at one gene, systems genetics explores one or more quantitative trait loci (QTL) associated with a given disease or complex trait. QTLs are regions within the genome whose genetic variation modulates quantitatively a phenotype characteristic of the particular trait under study (Lynch and Walsh, 1998). Determining the association between variations in specific disease phenotypes or a trait, with variations in genotypes of a reference population can be used to locate a QTL. One of the methods used for mapping QTLs associated with complex traits is genetic markers-trait association. Genetic markers associated with certain loci can be inherited in linkage disequilibrium. Generating populations with linked loci in disequilibrium is achieved though either crosses between inbred lines, or use of the out-bred populations. In this study we used recombinant inbred mouse strains that are fully genotyped as a genetic reference population for mapping QTLs.

Recombinant inbred (RI) and advanced RI (ARI) mice are a genetically diverse, segregating reference population that affords a powerful tool for systems genetics approaches (Williams et al., 2001; Peirce et al., 2004; Tsaih et al., 2005). RI and ARI strains have been successfully used to map quantitative trait loci (QTLs) associated with various phenotypes and diseases (Hardy et al., 2001; Grizzle et al., 2002; Chesler et al., 2005; Miyairi et al., 2007; Abdeltawab et al., 2008). RI and ARI strains of mice are the progeny of two ancestral inbred strains. For example, BXD strains are progeny of mating $\mathrm{C} 57 \mathrm{Bl} / 6 \mathrm{~J}$ (B6) and DBA/2J (D2). B6 was mated with D2 to give F1 mice; all F1 progeny are identical, yet heterozygous, with one allele from the B6 parent $(B)$ and the other from the D2 parent $(D)$. Those F1 hybrids were then crossed to generate an F2 progeny, each with random patterns of recombination. Pairs of F2 mice were designated as "parents" of an RI line, and are then sib-mated for $>20$ generations to achieve homozygosity for each genetically distinct RI line (Figure 1-2). This breeding strategy accumulates recombinations and random assortment of polymorphic loci. The breeding scheme used for generation of ARI strains is slightly different from RI strains and was adopted to increase recombination events per ARI strain, resulting in roughly double the number of recombinations per strain compared to a conventional RI strain (Williams et al., 2001; Tsaih et al., 2005; Shifman et al., 2006). ARI breeding scheme involves random matting 
Figure 1-2. Breeding scheme used for generation of RI strains of mice.

BXD strains are an example of recombinant inbred mice strains (RI). In this Figure, a scheme of crossing C57B1/6J (B6 carrying B allele) female to a DBA/2J (D2 carrying D allele) male is shown. A representative one chromosome is shown. The parental strains chromosomes are homozygous and when crossed, their F1 progeny are identical and each chromosome pair is heterozygous carrying BD alleles. F1 mice are crossed to generate F2 progeny with random recombinations. Randomly assigned F2 pairs are designated as parents for a BXD strain, and are sib-mated for $\geq 20$ generations to achieve homozygosity. Advanced RI (ARI) strains are bred using a slightly different scheme, where at the F2 stage, mice are randomly mated till generation 9 or 11 (according to the adopted breeding scheme (Peirce et al., 2004)) avoiding sib mating and even cousin mating to increase the number of recombinations and diversity of the genetic pool. There are currently $>80$ ARI-BXD strains available for genome-wide association studies. 


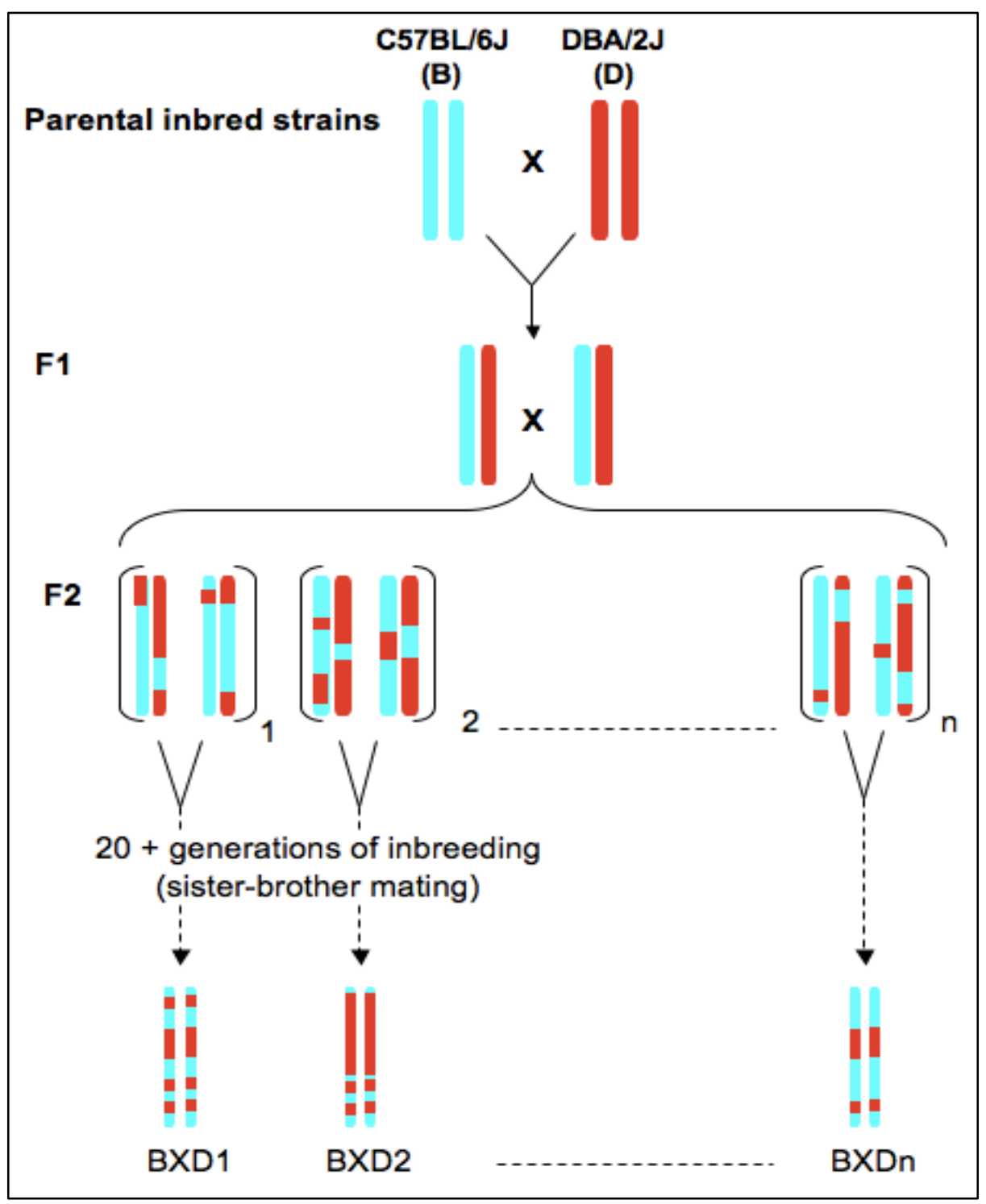


of F2 mice till generation 9 or 11 avoiding sib mating and even cousin matting to increase the recombinations and hence diversity of the genetic pool. After reaching generation 9 or 11, pairs of mice are set as parental for a designated BXD strain and are sib matted for $\sim 20$ generations or until each chromosome become homozygous (Peirce et al., 2004). The net result in both cases is a mosaic chromosomal pattern of both ancestral parents, B6 and D2.

In the studies presented in this dissertation, we used the BXD panel of RI and ARI strains. We had found that parental strains of BXD mice, B6 and D2 strains, showed differential response to GAS severe sepsis. We found that D2 were susceptible to severe GAS sepsis showing high mortality and bacterial loads in blood while B6 were relatively resistant. This differential response of the BXD ancestral strains to GAS sepsis motivated us to use ARI-BXD strains to parse out genes associated with differential response to GAS sepsis. Figure 1-2 shows the breeding scheme used to generate the BXD RI panel (explained above). The BXD strains are derived from B6 and D2 strains and consisting of homozygous, inbred lines, each of which is genetically distinct. There are now more than 80 BXD strains. Each strain is a clone, as the recombinations become fixed after approximately 20 generations of sib matting, so each strain is renewable and the number of mice that can be tested per strain can be infinite with high reproducibility of results. This is in contrast to using F2 crosses, which have recombinations and have been used in mapping studies, however, F2 crosses recombinations are random, and therefore, it may be not possible to reproduce results obtained using these mice. In contrast, each BXD strain has a unique set of recombinations different from the other BXD strains and is renewable, where we can test the same BXD strain several times $(n=\infty)$ with highly reproducible results. The high number of recombinations of each ARI-BXD strain makes these strains genetically diverse, thus mimicking the genetic diversity of the human population (Williams et al., 2001; Churchill, 2007; Peters et al., 2007). The use of BXD strains as a reference population is facilitated by the fact that BXD strains have been heavily genotyped with approximately 580,000 SNP and microsatellite markers (Churchill and Doerge, 1994; Shifman et al., 2006). In addition, the genomes of the B6

and D2 parental strains have been fully sequenced with 4.5 million SNPs, 450,000 indels, copy number variants, and inversions, facilitating the dissection of mapped genetic loci. All taken together makes the BXD panel of ARI strains a very powerful tool as a genetically diverse reference population for genome-wide association studies.

\section{Overview of Aims, Results, and Organization of the Dissertation}

\section{Aims of the Studies}

The overall aim of this dissertation was to analyze the role played by host immunogenetic variability in influencing differential susceptibility to severe GAS sepsis. To accomplish this overall aim, we first wanted to discover loci associated with differential genetic susceptibility to severe GAS sepsis. This was done by performing genome-wide scans for mapping QTLs associated with differential survival of a panel of recombinant inbred (RI) mice. These scans revealed multiple QTLs on mouse 
chromosomes (Chr) 2 and X. Next, steps were taken to fine tune and explore additional and interactive QTLs modulating severity of GAS sepsis. We fine-tuned the mapped QTLs to narrow down the list of candidate genes at mapped QTLs. To narrow down the candidate genes within individual mapped QTLs, we used additional BXD RI strains; chosen based on strains haplotypes also known as strain distribution patterns (SDP) of all BXD in the region of the mapped QTLs.

We then applied bioinformatics tools including linkage, gene ontology, cocitation networks, polymorphism analyses and pathway analyses to prioritize our candidate genes list. Next we aimed to identify genes and pathways modulating host response to GAS sepsis and validate those specific genes. This was accomplished by multiple approaches. We first compared the gene expression profiles of resistant and susceptible strains pre- and post- infection and validated differentially expressed gene identified with quantitative real time PCR analyses that revealed multiple pathways involved in modulating differential susceptibility to GAS sepsis. We then validated these pathways by studying the kinetics of soluble biomarkers, both cytokines and lipid mediators, associated with these pathways. We then aimed to analyze the cellular populations modulating differential response to severe sepsis and identify cellular mechanisms of differential response to severe GAS sepsis. The results of the studies are outline below including organization of the dissertation.

To identify correlates for survival, we explored additional independent and/or interactive traits associated with severity of GAS sepsis. Accordingly, we assessed variations in the number of bacteria in blood as well as dissemination of bacteria to various organs. Interestingly, both traits mapped to the same QTLs on Chr 2 associated with modulating survival but not with the one on Chr X. Additionally we found that there were interactions (epistatic effects) between two mapped QTLs on Chr 2. We also found interactions between Chr 2 QTLs and the Chr X QTL.

\section{Results and Organization of the Dissertation}

In Chapter 2 of this dissertation, I will discuss the establishment of the mouse model of GAS sepsis where we analyzed genetic, environmental and non-genetic covariates that might be contributing to the measured phenotypes. We standardized our model so that the observed phenotypes were mainly due to genetic differences of the strains (heritability) rather than any of the underlying covariates. The covariates studied included mice age, sex, and body weight, in addition to bacterial inoculate. This study is crucial, as phenotypes associated with complex traits can be affected by interactions with environment and/or other covariates, thus masking the actual gene networks associated with the trait under study. Chapter 2 was published as a peer-reviewed manuscript: Aziz, R.K., Kansal, R., Abdeltawab, N.F., Rowe, S.L., Su, Y., Carrigan, D., Nooh, M.M., Attia, R.R., Brannen, C., Gardner, L.A. et al (2007) Susceptibility to severe Streptococcal sepsis: use of a large set of isogenic mouse lines to study genetic \& environmental factors. Genes Immun. 8(5): 404-415. 
In the next chapter (Chapter 3), I will present how we analyzed disease phenotypes in the context of the mice genotypes which mapped two quantitative trait loci (QTLs) on Chromosome (Chr) 2. One QTL, between 22-35 Mb, that strongly predicts disease severity and accounts for $25 \%-30 \%$ of variance (calculated using hereditary tests) was highly significant (likelihood ratio statistic (LRS) 34.2 i.e. $\mathrm{P}<0.0000001$ ). We also mapped disease severity to a second QTL on Chr 2 (LRS 12 P $<0.001$ ). Both QTLs harbor several polymorphic genes known to regulate immune responses to bacterial infections. Candidate genes within these QTLs were evaluated using multiple parameters that included linkage, gene ontology, and variation in gene expression. We identified interleukin 1 (IL1) and prostaglandin E synthases pathways as key networks involved in modulating GAS sepsis severity. Chapter 3 was published as a peer-reviewed manuscript: Abdeltawab, N., Aziz, R.K., Kansal, R.K., Rowe, S., Su, Y., Gardner, L.A., Brannen, C., Nooh, M., Attia, R., Abdelsamed, H. et al. (2008). An Unbiased Systems Genetics Approach to Mapping Genetic Loci Modulating Susceptibility to Severe Streptococcal Sepsis. PLoS Pathog. 4(4):e1000042.

In Chapter 4, I will present our attempts to identify molecular and soluble biomarkers associated with differential susceptibility at multiple stages of infection. I explored changes in the genome-wide expression levels, where we examined differential gene expression at early stages of infection in resistant and susceptible strains and compared it to late stages of infection. In addition, we sought to examine patterns of cytokines, as soluble biomarkers, associated with late and early stages. Chapter 4 is a manuscript in preparation: Abdeltawab, NF, Kansal, R, Mukandan, S., Bangar, H., Aronoff, D., Senn, T., Jordan, M., Williams, RW, \& Kotb, M. Genomic \& soluble biomarkers associated with different outcomes of Group A streptococcal sepsis reveal underlying mechanisms of differential host susceptibility.

Next, in Chapter 5 we wanted to examine the role of these genes and pathways at the cellular level. We immunophenotyped selected resistant and susceptible BXD strains where we examined and characterized the relative percentages of blood and spleen cellular populations at normal uninfected selected resistant and susceptible strains. We concluded that migratory and functional differences rather than cellular availability might be a possible mechanism of resistance. Subsequently, we wanted to dissect and confirm the cellular response in our model by depleting neutrophils, which are known to be associated with susceptibility to GAS infection. We blocked Gr-1 receptors, which are receptors expressed on the surface of neutrophils and some populations of monocytes and dendritic cells, this rendered resistant strains susceptible. This led us to more experiments on analyzing the role of neutrophils and the associated host pathways modulating this response. Chapter 5 is a manuscript in preparation: Abdeltawab, NF, Bangar, H., Kansal, R., Williams, RW, \& Kotb, M. Host-pathogen interactions in Group A streptococcal sepsis: role of macrophages $\&$ neutrophils.

Finally, I will discuss and summarize the results of the studies done in this dissertation in Chapter 6, in addition to future directions of this project. 


\title{
CHAPTER 2. SUSCEPTIBILITY TO SEVERE STREPTOCOCCAL SEPSIS: USE OF A LARGE SET OF ISOGENIC MOUSE LINES TO STUDY GENETIC AND ENVIRONMENTAL FACTORS*
}

\begin{abstract}
Summary
Variation in responses to pathogens is influenced by exposure history, environment, and the host's genetic status. We recently demonstrated that HLA class II allelic differences are a major determinant of the severity of invasive group A streptococcal (GAS) sepsis in humans. While in-depth controlled molecular studies on populations of genetically well-characterized humans are not feasible, it is now possible to exploit genetically diverse panels of recombinant inbred (RI) BXD mice to define genetic and environmental risk factors. Our goal in this study was to standardize the model and identify genetic and nongenetic covariates influencing invasive infection outcomes. Despite having common ancestors, the various BXD strains ( $\mathrm{n}$ strains $=33, \mathrm{n}$ individuals $=445$ ) showed marked differences in survival. Mice from all strains developed bacteremia but exhibited considerable differences in disease severity, bacterial dissemination, and mortality rates. Bacteremia and survival showed the expected negative correlation. Among nongenetic factors, age — but not sex or weight—was a significant predictor of survival $(\mathrm{p}=0.0005)$. To minimize nongenetic variability, we limited further analyses to mice ages 40-120 days and calculated a corrected relative survival index (cRSI) that reflects the number of days an animal survived post-infection normalized to all significant covariates. Genetic background (strain) was the most significant factor determining susceptibility ( $\mathrm{p} \leq 0.0001$ ), thus underscoring the strong effect of host genetic variation in determining susceptibility to severe GAS sepsis. This model offers powerful unbiased forward genetics to map specific quantitative trait loci and networks of pathways modulating the severity of GAS sepsis.
\end{abstract}

\section{Introduction}

There is now overwhelming evidence that specific genetic factors influence a host's susceptibility or immunity to many infectious agents, and modulate severity and outcome of infectious diseases (reviewed in [Skamene, 1983; Roy and Malo, 2002; Frodsham and Hill, 2004; Kotb, 2004]). Infection with group A streptococcus (GAS or Streptococcus pyogenes) represents an ideal model for studying effects of host genetics on disease. This human pathogen causes a wide variety of conditions ranging from sore throat to invasive life-threatening diseases (reviewed in [Cunningham, 2000]), and the same strain of pathogen leads to different outcomes in different individuals (Chatellier et $a l ., 2000)$. Our laboratory provided direct evidence that the highly polymorphic human

\footnotetext{
* Source: Reprinted with permission. Aziz, R.K., Kansal, R., Abdeltawab, N.F., Rowe, S.L., Su, Y., Carrigan, D., Nooh, M.M., et al. (2007) Susceptibility to severe Streptococcal sepsis: use of a large set of isogenic mouse lines to study genetic and environmental factors. Genes Immun 8: 404-415.
} 
leukocyte antigen (HLA) genes confer high risk or protection in rheumatic heart disease (Guedez et al., 1999) as well as in streptococcal toxic shock (STSS) and necrotizing fasciitis (NF) (Kotb et al., 2002; Kotb et al., 2003). In GAS sepsis, allelic variation in HLA class II molecules plays a major role in modulating infection severity, primarily because they serve as binding and signaling receptors for superantigens (Kotb, 1992; Kotb, 1995), which are the primary trigger of the severe inflammatory responses that can lead to organ failure and shock (Norrby-Teglund et al., 2001; Proft et al., 2003).

To systematically identify additional gene variants that modulate susceptibility to severe GAS sepsis and to elucidate how they influence disease outcome, we sought a robust experimental model of the disease with attributes that incorporate roughly the same level of genetic variation as that of human populations. Small numbers of conventional inbred mouse strains have been used previously to study differential susceptibility to GAS infection (Medina et al., 2001; Goldmann et al., 2003, 2004b); however, these models are limited in their genetic variability and cannot be used to map modifier loci. HLA transgenic mice are appropriate models for GAS sepsis (Nooh et al., 2007) but these too are generated on the genetic background of conventional inbred laboratory strains.

The genetically diverse mouse reference population of recombinant inbred (RI) strains, which can be likened to an immortal population of human monozygotic twins, is an ideal model for our studies. RI strains are generated by the crossing of two inbred strains followed by $\geq 20$ consecutive generations mating among siblings (Bailey, 1971; Taylor, 1978). These types of strains are frequently used for studying genetic variation and mapping quantitative trait loci (QTL) influencing disease (Peirce et al., 2004; Chesler et al., 2005). The BXD strains were used recently to study variation in the pathogenicity of bovine spongiform encephalopathy and to uncover genes modulating anthrax and pox lethality (Benjamin et al., 1986; Melvold et al., 1990; Watters et al., 2001) Among the different panels of RI strains, we selected the BXD set produced from an intercross of $\mathrm{C} 57 \mathrm{BL} / 6 \mathrm{~J}$ and $\mathrm{DBA} / 2 \mathrm{~J}$. The parental strains are known to differ in response to many pathogens (Plant and Glynn, 1976; Anthony et al., 1989; Boyartchuk et al., 2001; Mahler et al., 2002). The other notable advantage of this particular panel of RI strains is that both parental strains have been sequenced (Waterston et al., 2002). This makes it possible to use reverse genetic methods to test modifier genes (Williams, 2006). We have 80 BXD strains that are being extensively phenotyped and genotyped.

As a first step in exploiting the BXD strains to study the genetics of GAS susceptibility, we have studied the influence of differences in sex, age, body weight, inoculum titer, and genetic factors on disease development. Our main objective has been to develop a protocol that can be applied uniformly across the entire BXD set of 80 . Our second objective has been to measure heritability and evaluate prospects of mapping modifier loci. We show the genetic and nongenetic factors that determine whether a mouse is protected from or susceptible severe GAS sepsis, we evaluate the contribution of each of these factors using multivariate analysis, and we discuss the proper measures that should be followed to design experiments using this novel and promising mouse 
model in other infectious disease and for elucidating the complex events of host-pathogen interactions.

\section{Results}

The main purpose of this study was to adapt and standardize the BXD RI model to investigate the genetic and molecular mechanisms associated with susceptibility to severe invasive GAS sepsis, to determine the nongenetic environmental factors affecting the model, and to test whether the genetic variations among these mice would affect the progression and severity of invasive GAS disease. We used 717 mice in the study: 218 mice in the preliminary phase, 445 for the initial statistical analysis, 307 of which were selected for the final analysis as detailed below, and 54 for the time-course experiments.

\section{Survival Scores Show Differential Susceptibility to Severe GAS Sepsis}

To assess the phenotype 'susceptibility or resistance of mice to severe GAS sepsis,' we calculated a survival score for each animal as a quantitative trait: among 445 mice tested in six experiments, a notable variability was observed in survival scores measured as days post-infection. Interestingly, the distribution of the survival scores was not normal but multimodal, showing three groups (Figure 2-1-A), the largest of which represented animals $(n=208)$ that died early (in less than 2.5 days). Another group included an intermediate population $(n=125)$ that survived between 2.5 and 4.5 days and the third group $(n=112)$ survived for more than 5 days and up to the entire 7-day observation period. This pattern of survival was highly reproducible and predictable from one experiment to another with slight variability in the boundaries separating the three clusters in each experiment.

\section{Bacterial Loads in Blood and Organs Show Various Degrees of Bacteremia and Tissue Dissemination}

The bacterial load in blood (calculated as 'bacteremia index,' $\mathrm{BI}=\log \mathrm{CFU} / \mathrm{ml}$ blood sampled $24 \mathrm{~h}$ post-infection) was measured for 396 of 445 mice used in six experiments. In contrast to the survival scores, bacterial load followed a normal distribution (Figure 2-1-B). With the exception of a few mice $(n=6)$ whose blood was sterile (possibly due to injection failure), all mice had bacteremia with counts ranging from $125 \mathrm{CFU} / \mathrm{ml}(\mathrm{BI}=2.097)$ to $1.72 \times 10^{9} \mathrm{CFU} / \mathrm{ml}(\mathrm{BI}=9.236)$, the mean $\mathrm{BI} \pm \mathrm{SE}$ was $5.2 \pm 0.07$ reflecting a highly symmetrical distribution. Correlation analysis showed an inverse relation between survival scores and BI. The Pearson product moment-correlation factor of -0.430 (Figure 2-1-C) indicates that the BI accounts for $20-30 \%$ of variance in mortality.

\footnotetext{
* In some cases, mice died before their blood was sampled and in other cases, the bacteria were too numerous to be counted by the plate dilution technique.
} 

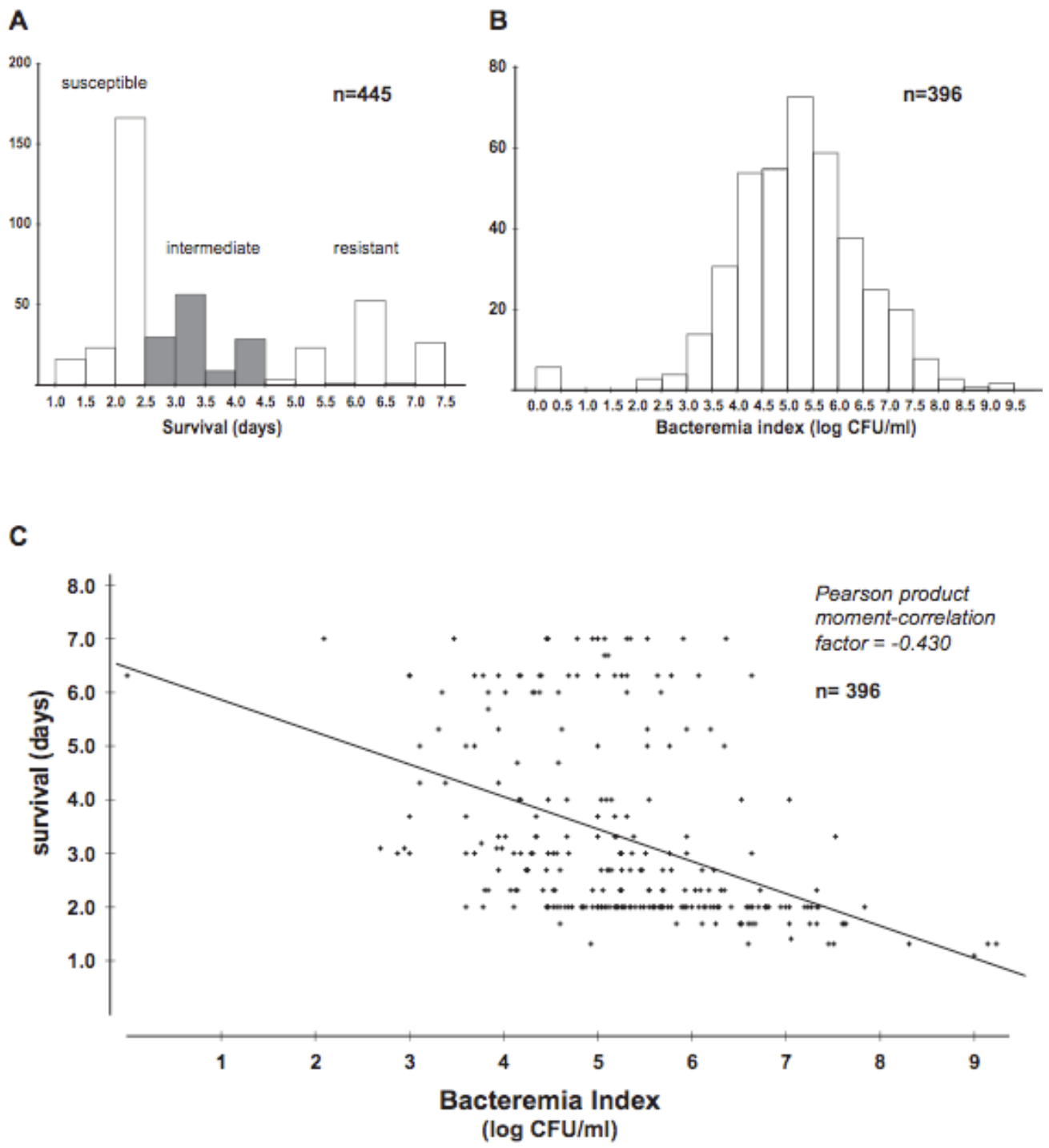

Figure 2-1. Distribution and correlation of survival scores and bacteremia indices.

Histograms showing the frequencies of survival scores (A) and log CFU/ml blood, or bacteremia indices, BI (B). A. The distribution of the survival scores of 445 animals from six experiments was multimodal, showing three clusters representing a susceptible, an intermediate, and a resistant population. Bars representing the intermediate population are shaded in grey. B. The distribution of BI for 396 was unimodal and symmetric, with the exception of six animals having sterile blood (leftmost bar). C. A scatter plot showing the negative correlation between survival scores and BI. Pearson productmoment correlation factor is indicated. 
In addition to counting bacteria in blood, in some experiments we determined the bacterial dissemination in different systemic organs (mainly spleen, but also liver and lung) to estimate invasiveness of the disease. Among 335 mice with confirmed bacteremia, only 14 had sterile spleens while the remaining mice had bacterial counts ranging from 102 to $3 \times 10^{10} \mathrm{CFU} /$ spleen, with a median count of $1.3 \times 10^{8} \mathrm{CFU} / \mathrm{spleen}$. Bacterial loads in liver and lung were determined for 77 mice, and the median counts were $3.16 \times 10^{9} \mathrm{CFU} /$ liver and $2 \times 10^{9} \mathrm{CFU} /$ lung, with a wide range of variation.

\section{Statistical Analysis Evaluates the Confounding Factors Affecting Mice Survival}

The variability in animal mortality and extent of bacteremia is not surprising and matches that reported in human infection (Davies et al., 1996; Basma et al., 1999; Muller et al., 2003) and in mice strains (Medina et al., 2001). However, in this particular set of experiments, it was necessary to determine whether this variability reflects genetic differences between different BXD strains or is it mere biological variability caused by confounding factors like sex, body weight, animal age, or experiment-to-experiment variability (due in part to differences in inoculum size or other experimental error). The large number of animals used and the availability of replenishable inbred BXD lines made such analysis possible and efficient.

First, we examined the effect of inoculum size. In preliminary experiments, we conducted a dose-response study in which we exposed animals to bacterial inocula ranging from $10^{5}$ to $10^{8} \mathrm{CFU} / \mathrm{ml}$ to find an optimal dose that would kill animals rapidly but would also discriminate between susceptible and resistant strains (data not shown). We chose an inoculum size of $10^{7} \mathrm{CFU} / \mathrm{ml}$ because it showed clear differences between the parental strains $(\mathrm{C} 57 \mathrm{BL} / 6 \mathrm{~J}$ and DBA/2J). However, because it is difficult to reproduce the inoculum titer in every experiment, and because precise counts could only be determined after injection, it was important to examine whether the inter-experimental differences in inoculum size had an effect on survival. Slight variation in inoculum size had an insignificant effect on survival ( $\mathrm{r} 2=0.1 \%, \mathrm{p}=0.533$ ) Figure 2-2-A.

We tested whether sex had an effect on survival. Male mice $(\mathrm{n}=235)$ had a mean survival of $3.49 \pm 0.12$ days and a median of 3 days, slightly higher than female mice (n $=206)$ that had a mean of $3.25 \pm 0.12$ days and a median of 2.3 days. This difference did not achieve statistical significance $(\mathrm{p}=0.153)$ Figure 2-2-B.

Subsequently, we tested the effect of animal body weight and age on survival, and each of the two factors, independently, showed positive association with survival scores (Figure 2-2-C and 2-2-D). Whereas weight had a correlation of 0.29 , age has a stronger correlation (factor $=0.44$ ). It is noteworthy that weight was directly proportional with animal age between the ages of 40-120 days. Based on these findings, we decided to use animals in the age range of 40-120 days and to correct for age effects using a General Linear Model (GLM). 

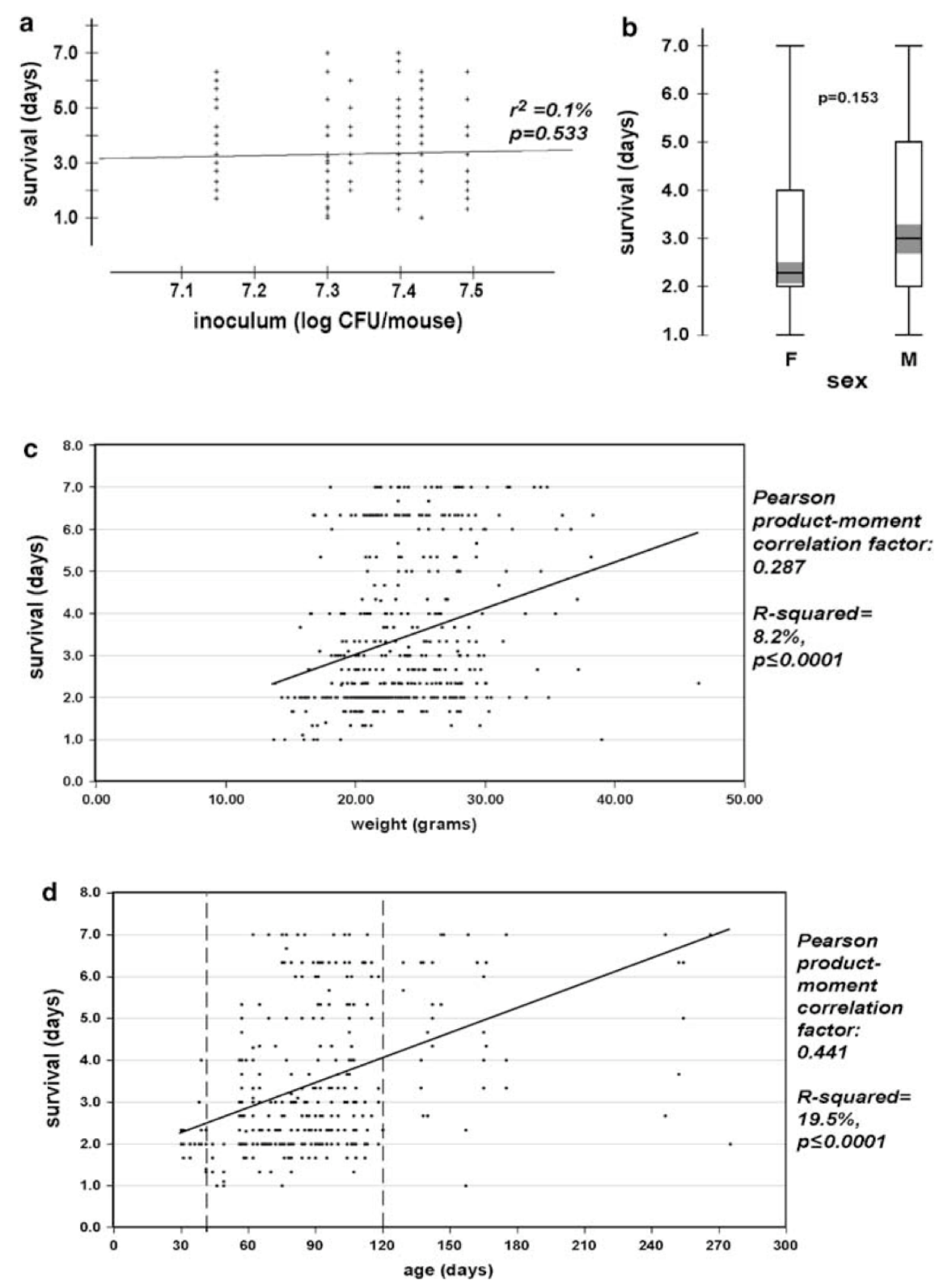

\section{Figure 2-2. Effect of non-genetic factors on the survival of BXD mice and their parental strains.}

Scatter plots showing the effect of the inoculum size (A), the mice body weight (C), and their age (D) on their survival. The effects of these confounding factors were evaluated by linear regression and Pearson product-moment correlation analyses, and the values of $\mathrm{r} 2$ and correlation factors are shown when necessary. B. Box plots of animal sex versus animal survival in days post-infection. The significance of the effect of sex was tested by the student-t test, and the p-value is shown. D. Vertical dashed lines show the range of animals selected for further analysis. 


\section{Analysis of Covariates Underscores the Role of Genetic Background in Determining Animal Susceptibility to GAS}

Having determined the effect of several major cofactors on survival scores, we set out to determine the complex interaction between these covariates and the genetic factor (i.e., the mouse strain). At this stage of the analysis, we filtered our data set to exclude mice that were outside the 40-120 days age range (see above) and we selected BXD strains that had been used in at least two experiments. Table 2-1 lists mice selected for final analysis. We took advantage of the multimodal distribution of survival scores detailed above (see Figure 2-1-A) to define a survival index (SI): animals were given a score ranging from 0.25 to 3 , with increments of 0.25 ( 0.25 for most susceptible and 3.0 for most resistant). The use of SI was superior to survival scores as it normalized the data across experiments and minimized the unavoidable experiment-to-experiment variance in survival scores caused by slight differences in inoculum size and other systematic sources of error.

Finally, we performed GLM analysis of covariates using ordinary least squares ANOVA to determine the relative effect of covariates, including mouse strain, on survival (Table 2-2). This analysis revealed that strain was the most significant predictor $(p \leq 0.0001)$, followed by age $(p=0.0005)$, and weight $(p=0.02)$. As shown in Figure 2$2-\mathrm{B}$, sex remained an insignificant factor in the final analysis (Table 2-2). From the GLM, we estimate the main effect attributable to strain (a measure of heritability) to be 25-30\% (42.21/152.44, see Table 2-2). This GLM analysis was not only helpful in assessing effects of several factors, but it also allowed us to generate a corrected relative survival index (cRSI), which was used to compare the strains and determine their relative susceptibility — correcting for differences in age and weight (Figure 2-3).

\section{BXD Strains Exhibited Phenotype Variability Outside the Range of the Parental Strains}

The comparison of cRSI values between different strains (Figure 2-3) reveals the stark effect that the genetic background plays in predicting how long a mouse will survive after being exposed to a high dose of GAS. Some strains have very low mean cRSI (e.g., BXD66, BXD97, and BXD61) and are highly susceptible, whereas others are almost completely resistant (e.g., BXD87, BXD92, and BXD69). The difference between the cRSI values of parental strains, shown on the two extreme sides of the bar chart (Figure 2-3), was more modest than the variation displayed by the different BXD strains. This finding underscores the complexity of the trait we are measuring (susceptibility/ resistance) and highlights the fact that the survival phenotype is modulated by multiple loci.

Following the same method of building a GLM for covariate analysis, we corrected the $\mathrm{BI}$ and the log $\mathrm{CFU} /$ spleen by calculating the coefficients that express the contribution of 'mouse strain' as a predictor for BI and for spleen dissemination (Figure 2-4). As shown with the inverse correlation between survival score and BI (Figure 2-1-C), most strains that were judged susceptible based on their cRSI (e.g. BXD60, 
Table 2-1. Mouse strains used in the final analysis of this study.

\begin{tabular}{lccl}
\hline Strain & N & $\begin{array}{c}\text { Age } \\
(\text { Mean } \pm \text { SE) }\end{array}$ & $\begin{array}{c}\text { Body Weight } \\
\text { (Mean } \pm \text { SE) }\end{array}$ \\
\hline C57BL/6J & 11 & $75.55 \pm 3.76$ & $21.98 \pm 0.90$ \\
DBA/2J & 12 & $88.92 \pm 3.79$ & $23.74 \pm 0.59$ \\
BXD43 & 11 & $91.45 \pm 5.05$ & $23.92 \pm 0.77$ \\
BXD44 & 11 & $75.09 \pm 7.96$ & $22.67 \pm 1.88$ \\
BXD45 & 20 & $80.00 \pm 4.48$ & $24.10 \pm 0.72$ \\
BXD51 & 16 & $74.00 \pm 4.39$ & $23.32 \pm 0.89$ \\
BXD60 & 13 & $68.38 \pm 6.31$ & $24.28 \pm 1.63$ \\
BXD61 & 9 & $57.67 \pm 0.53$ & $20.13 \pm 0.64$ \\
BXD62 & 14 & $62.14 \pm 2.26$ & $20.70 \pm 0.71$ \\
BXD64 & 12 & $96.67 \pm 2.38$ & $22.46 \pm 0.77$ \\
BXD66 & 7 & $90.57 \pm 6.30$ & $23.66 \pm 1.19$ \\
BXD69 & 14 & $73.93 \pm 5.63$ & $21.48 \pm 0.70$ \\
BXD70 & 17 & $82.00 \pm 4.83$ & $24.11 \pm 0.81$ \\
BXD73 & 14 & $86.14 \pm 2.01$ & $22.40 \pm 1.24$ \\
BXD74 & 14 & $77.00 \pm 7.14$ & $21.53 \pm 0.97$ \\
BXD75 & 22 & $74.36 \pm 4.16$ & $23.69 \pm 0.74$ \\
BXD85 & 16 & $78.69 \pm 4.97$ & $26.72 \pm 1.42$ \\
BXD87 & 8 & $89.00 \pm 3.78$ & $19.50 \pm 0.74$ \\
BXD89 & 23 & $87.22 \pm 4.48$ & $23.71 \pm 0.56$ \\
BXD90 & 17 & $83.71 \pm 5.09$ & $27.40 \pm 0.81$ \\
BXD92 & 14 & $81.86 \pm 1.82$ & $27.48 \pm 1.66$ \\
BXD97 & 12 & $98.33 \pm 4.24$ & $26.68 \pm 1.09$ \\
Total Cases & $\mathbf{3 0 7}$ & $\mathbf{8 0 . 3 3} \pm \mathbf{1 . 1 2 3}$ & $\mathbf{2 3 . 6 6} \pm \mathbf{0 . 2 4 0}$ \\
\hline
\end{tabular}

Table 2-2. Analysis of Variance for Survival Index.

\begin{tabular}{lllllr}
\hline Source & df* & $\begin{array}{l}\text { Sums of } \\
\text { Squares }\end{array}$ & $\begin{array}{l}\text { Mean } \\
\text { Square }\end{array}$ & F-ratio & Probability \\
\hline Const & 1 & 937.56 & 937.56 & 2911.80 & $\leq 0.0001$ \\
Wt & 1 & 1.73 & 1.73 & 5.37 & 0.0212 \\
Sex & 1 & 0.19 & 0.19 & 0.58 & 0.4452 \\
Age & 1 & 3.97 & 3.97 & 12.32 & 0.0005 \\
Strain & 21 & 42.21 & 2.01 & 6.24 & $\leq 0.0001$ \\
Error & 282 & 90.80 & 0.32 & & \\
Total & $\mathbf{3 0 6}$ & $\mathbf{1 5 2 . 4 4}$ & & & \\
\hline
\end{tabular}

* df: Number of degrees of freedom. 


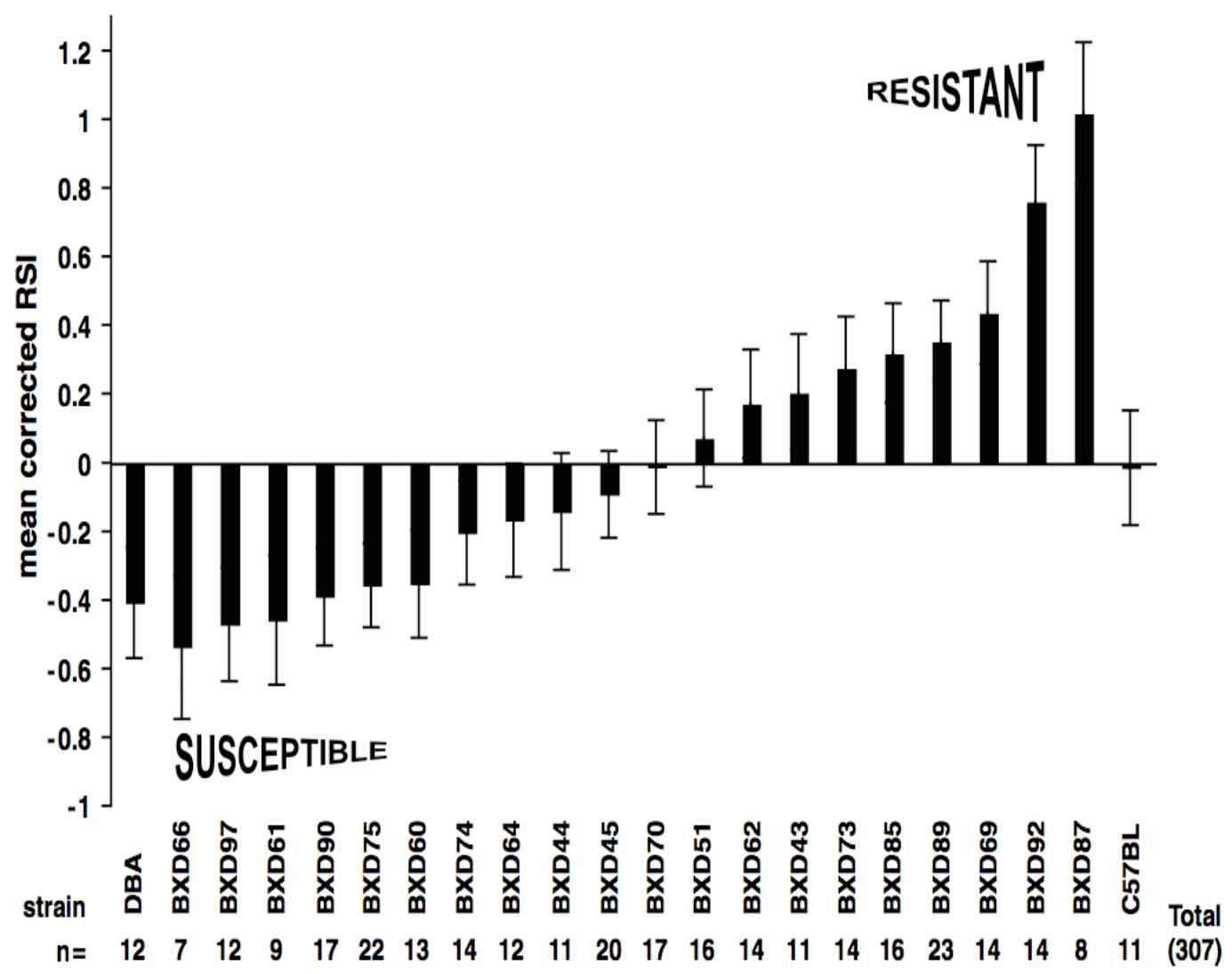

Figure 2-3. Differential susceptibility of BXD strains to invasive GAS.

A bar chart showing the mean values of corrected relative survival indices (cRSI) for different BXD strains, arranged in ascending order. Parental strains $(\mathrm{C} 57 \mathrm{Bl} / 6 \mathrm{~J}$ and $\mathrm{DBA} / 2 \mathrm{~J}$ ) are shown on the two extremities of the $\mathrm{X}$-axis. Error bars represent the standard errors of the means. The total number of animals (n) used per strain is indicated. 

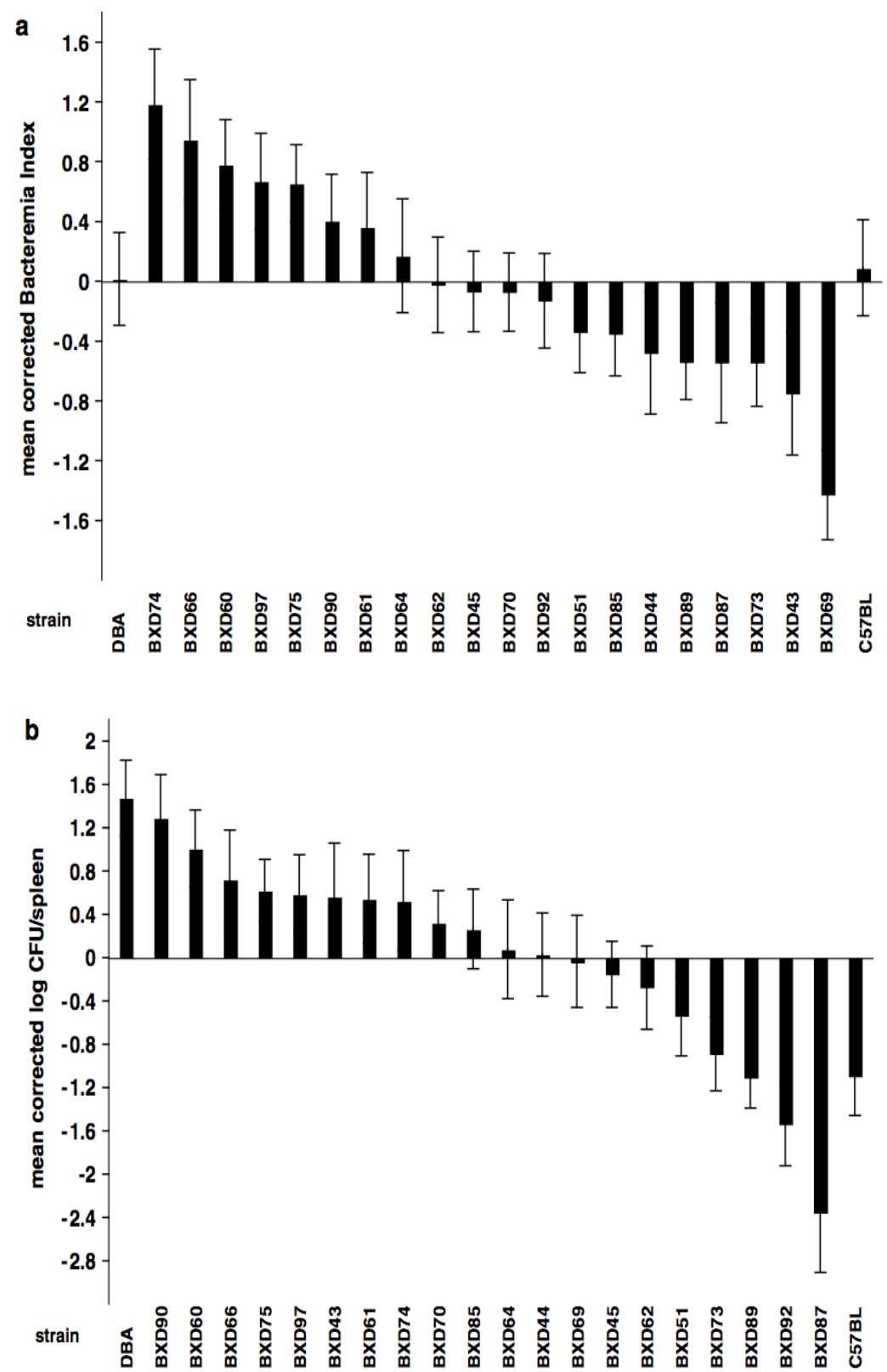

Figure 2-4. Variation in bacteremia and spleen dissemination in different BXD strains.

Bar chart showing the mean values of corrected bacteremia indices, cBI, (A) and corrected loc CFU/spleen (B) for different BXD strains, arranged in ascending order. Parental strains $(\mathrm{C} 57 \mathrm{Bl} / 6 \mathrm{~J}$ and $\mathrm{DBA} / 2 \mathrm{~J})$ are shown on the two extremities of the $\mathrm{X}$-axis. Error bars represent the standard errors of the means. 
BXD66, and BXD75) had higher cBIs, and vice versa (e.g. BXD69 and BXD73). However, there were exceptions, e.g., BXD44 had a negative cRSI and a negative cBI as well, and BXD92 was one of the most resistant strains but showed intermediate, rather than low, bacteremia (Figure 2-4-A). Additionally, when examining the bacterial dissemination in spleens of different strains (Figure 2-4-B), most susceptible strains had high dissemination and vice versa, again with exceptions, e.g. BXD69. Conversely, the parental strains were this time clearly on two sides of the spectrum, with DBA/2J having the highest spleen dissemination and $\mathrm{C} 57 \mathrm{BL} / 6 \mathrm{~J}$ having one of the lowest values.

Collectively, the data suggest the existence of different mechanisms leading to mortality.

\section{Controlled Time-course Experiments Show the Kinetics of Bacterial Spread and Confirm Results of Population-based Experiments}

Having generated data with large number of mice and with strong statistics, we moved to confirm the population-based study through time-course experiments that would clarify the kinetics of bacterial dissemination or clearance in the early hours of infection. In one representative experiment, we selected animals from six strains predicted to have the following descending order of susceptibility BXD61, BXD60, BXD51, BXD73, BXD89, BXD69 (Figure 2-3-A) and we infected them with the same dose of bacteria, then sacrificed three mice per strain at $18 \mathrm{~h}, 36 \mathrm{~h}$, and $60 \mathrm{~h}$. Overall, the results were as expected: All mice developed bacteremia, and their organs (spleen, lung, and liver) were positive for bacterial dissemination, but the kinetics of bacterial dissemination/ clearance were quite different. Strains BXD60 and BXD61, predicted as the most susceptible in this experiment, behaved as expected with highest bacterial loads in their spleens and highest rate of bacterial invasiveness, estimated from the slopes of bacterial dissemination in their organs over time (Figure 2-5 and Table 2-3). BXD69 was the most resistant strain to bacterial dissemination, with the lowest bacterial loads in organs and a tendency to clear bacteria rapidly (note the negative slopes in Figure 2-5-B and 2-5-C); this behavior matches its rank as having one of the lowest cRSI (Figure 2-3) and cBI (Figure 2-4-A). A similar pattern was displayed by BXD51, an intermediate strain that tends to have lower bacterial counts in blood and spleen (Figure 2-4). More interesting, however, was the behavior of BXD73 and BXD89 strains, both of which are resistant strains that showed delayed bacterial clearance in the first $60 \mathrm{~h}$. While BXD73 had bacterial dissemination in organs an order of magnitude lower than BXD60 and BXD61, BXD89's counts seemed to follow the pattern of a susceptible rather than a resistant strain. This underscores the dissociation between the speed of bacterial dissemination and animal mortality as separate traits. A possible explanation is that BXD89 has an attenuated immune system that may delay bacterial clearance but that does not harm the host.

\section{Preliminary Quantitative Trait Loci Mapping}

To provide accurate QTL mapping, we are conducting a more extensive study with more strains and more mice per strain. However, with the current number of strains 
Figure 2-5. Kinetics of bacterial dissemination in lung, liver, and spleen of six BXD strains.

Scatter plots of the bacterial dissemination during the first $60 \mathrm{~h}$ of an active infection in spleens (A), livers (B), and lungs (C) of mice from 6 strains with different susceptibility to GAS sepsis. Color regression lines indicate the rate and extent of change in $\log \mathrm{CFU}$ bacteria/organ versus time for each strain. 

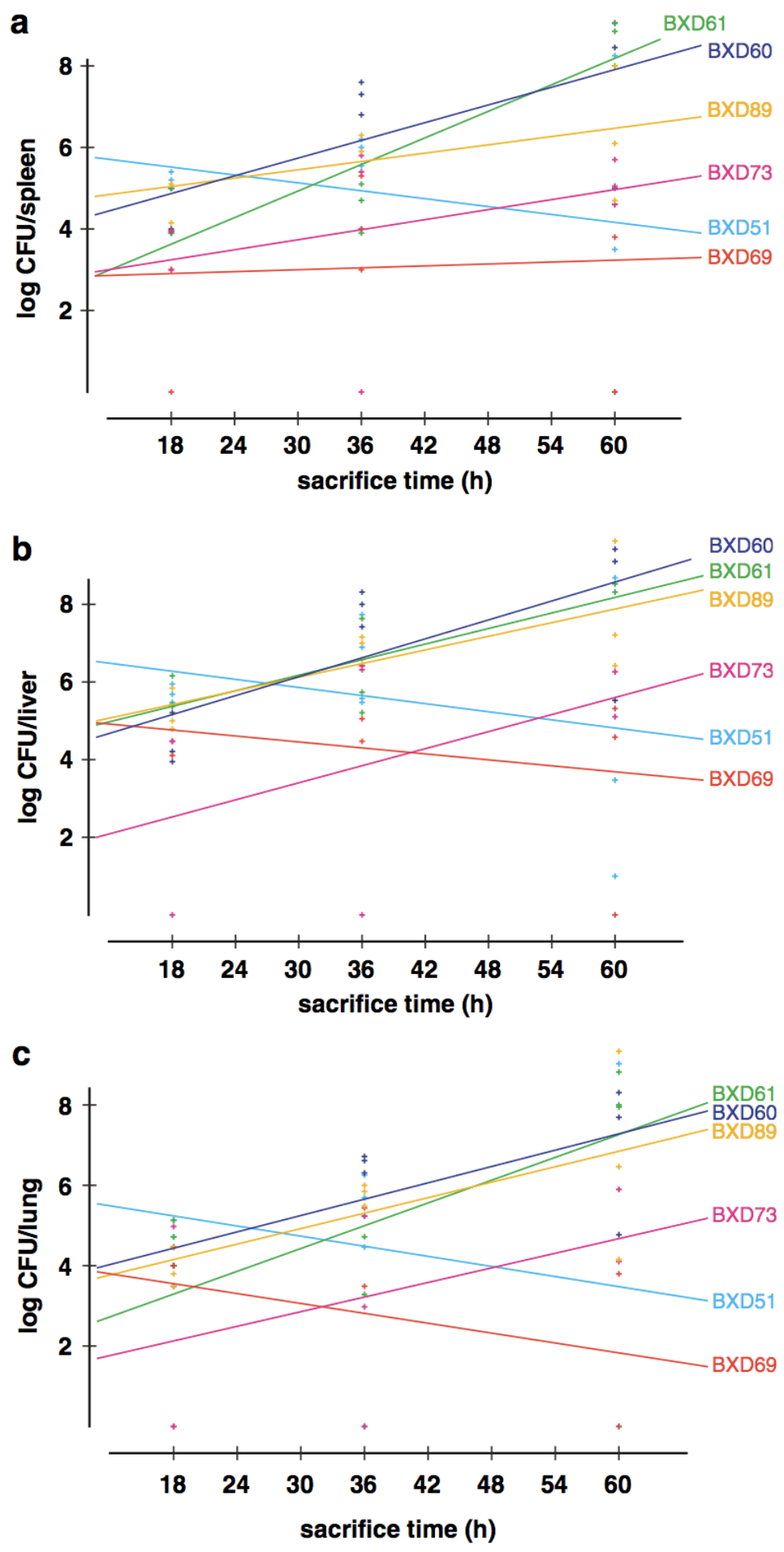
Table 2-3. Extent of invasiveness in time-course experiments, as measured by bacterial loads in spleen, liver, and lung.

\begin{tabular}{|c|c|c|c|c|c|}
\hline \multirow[b]{2}{*}{ Strain } & \multicolumn{3}{|c|}{ Mean \pm SE Log CFU/Spleen } & \multicolumn{2}{|c|}{ Regression } \\
\hline & $1 \overline{8 h}$ & $36 \mathrm{~h}$ & $\overline{60 h}$ & $\overline{\mathbf{r}^{2}}$ & $\bar{p}$ \\
\hline BXD51 & $5.19 \pm 0.12$ & $5.44 \pm 0.50$ & $3.91 \pm 2.39$ & $6.80 \%$ & 0.466 \\
\hline BXD60 & $4.29 \pm 0.35$ & $7.22 \pm 0.24$ & $7.50 \pm 1.26$ & $47.90 \%$ & 0.039 \\
\hline BXD61 & $4.26 \pm 0.37$ & $4.57 \pm 0.35$ & $8.64 \pm 0.32$ & $81.40 \%$ & 0.001 \\
\hline BXD69 & $2.31 \pm 1.19$ & $4.10 \pm 0.67$ & $2.79 \pm 1.41$ & $0.60 \%$ & 0.84 \\
\hline BXD73 & $3.48 \pm 0.48$ & $3.74 \pm 1.87$ & $5.11 \pm 0.32$ & $14.60 \%$ & 0.35 \\
\hline \multirow[t]{2}{*}{ BXD89 } & $4.78 \pm 0.31$ & $6.16 \pm 0.13$ & $6.27 \pm 0.96$ & $29.50 \%$ & 0.131 \\
\hline & \multicolumn{3}{|c|}{ Mean \pm SE Log CFU/Liver } & \multicolumn{2}{|c|}{ Regression } \\
\hline Strain & $18 \mathrm{~h}$ & 36h & $60 h$ & $\mathbf{r}^{2}$ & $\mathbf{p}$ \\
\hline BXD51 & $5.71 \pm 0.13$ & $6.43 \pm 0.54$ & $4.06 \pm 2.53$ & $9.60 \%$ & 0.385 \\
\hline BXD60 & $4.45 \pm 0.39$ & $7.92 \pm 0.26$ & $8.03 \pm 1.25$ & $49.00 \%$ & 0.036 \\
\hline BXD61 & $5.64 \pm 0.27$ & $6.20 \pm 0.74$ & $8.39 \pm 0.06$ & $72.70 \%$ & 0.004 \\
\hline BXD69 & $4.22 \pm 0.13$ & $5.31 \pm 0.58$ & $3.31 \pm 1.67$ & $6.80 \%$ & 0.499 \\
\hline BXD73 & $2.24 \pm 2.24$ & $4.24 \pm 2.12$ & $5.49 \pm 0.38$ & $23.80 \%$ & 0.22 \\
\hline \multirow[t]{2}{*}{ BXD89 } & $5.21 \pm 0.33$ & $6.92 \pm 0.16$ & $7.74 \pm 0.96$ & $56.50 \%$ & 0.02 \\
\hline & \multicolumn{3}{|c|}{ Mean \pm SE Log CFU/Lung } & \multicolumn{2}{|c|}{ Regression } \\
\hline Strain & $18 \mathrm{~h}$ & $36 h$ & $60 \mathrm{~h}$ & $\overline{\mathbf{r}^{2}}$ & $\overline{\mathbf{p}}$ \\
\hline BXD51 & $4.60 \pm 0.33$ & $5.43 \pm 0.38$ & $3.01 \pm 3.01$ & $7.00 \%$ & 0.46 \\
\hline BXD60 & $3.97 \pm 0.28$ & $6.55 \pm 0.13$ & $6.93 \pm 1.09$ & $52.20 \%$ & 0.028 \\
\hline BXD61 & $4.62 \pm 0.33$ & $2.68 \pm 1.40$ & $8.26 \pm 0.29$ & $39.00 \%$ & 0.072 \\
\hline BXD69 & $2.83 \pm 1.42$ & $4.13 \pm 0.65$ & $1.26 \pm 1.26$ & $12.70 \%$ & 0.346 \\
\hline BXD73 & $2.50 \pm 2.50$ & $2.74 \pm 1.52$ & $5.01 \pm 0.90$ & $19.10 \%$ & 0.327 \\
\hline BXD89 & $3.90 \pm 0.28$ & $5.77 \pm 0.16$ & $6.66 \pm 1.50$ & $42.00 \%$ & 0.059 \\
\hline
\end{tabular}


and mice we used the WebQTL tools on the GeneNetwork web site (http://www.genenetwork.org) and were able to exclude certain sites that could have possibly contributed to the modulation of GAS sepsis severity. For example, our human genetic association studies in patients with severe GAS sepsis revealed a strong association with HLA class II haplotypes (Kotb et al., 2002). It was therefore interesting to determine whether any QTLs map to the $\mathrm{H} 2$ locus. Although the parental strains differed in their $\mathrm{H} 2$ types (the C57/BL6 mice are H2-b while the DBA/2J mice are H2-d), we could not find any hint for significant QTLs in the H2 region on chromosome 17. Instead, our preliminary mapping results suggest two significant QTLs on chromosome 2 that we are currently verifying and fine-tuning using additional mice and strains. The insignificant contribution of the mice $\mathrm{H} 2$ locus was not entirely surprising because the HLA class II association in humans is related to the role of these molecules in presenting GAS superantigens to T-cells. While superantigens play a crucial role in the human disease and in HLA-transgenic mice (Nooh et al., 2007), they have little contribution in the BXD mouse model because GAS superantigens are poorly presented by mouse $\mathrm{H} 2$.

\section{Discussion}

\section{The Strength of the BXD Recombinant Inbred Population Model}

Clinical and epidemiological observations provide evidence of strong differences in human susceptibility to GAS infections and the severity and outcomes of diseases caused by the same strain of bacteria (Hoge et al., 1993; Davies et al., 1996; Kaul et al., 1997; Johnson et al., 2002; Kotb, 2004). For example, we have previously demonstrated a dominant effect of HLA class II allelic polymorphisms in severe GAS sepsis (Kotb et al., 2002; Kotb et al., 2003). We also provided biological confirmation of these associations with HLA both in vitro (Kotb et al., 2002; Norrby-Teglund et al., 2002) and in vivo (Nooh et al., 2007). Despite this strong association with HLA class II polymorphic alleles, we realize that sepsis is a complex disease affected by multiple interactive pathways. To identify additional pathways and polymorphisms contributing to the stark difference in the severity of GAS sepsis, we explored several animal models that may be suitable for this genetic study. While non-human primates represent an alternative to study human GAS infections (Virtaneva et al., 2003; Virtaneva et al., 2005), their use in immunogenetic population studies is expensive and impractical due to their slow breeding pattern and small progeny, high cost, and ethical issues. Meanwhile, regular laboratory mice, which share $\sim 85 \%$ gene content with humans (Jackson, 2001), are not sensitive to invasive GAS infections (Miethke et al., 1992; Welcher et al., 2002) just as certain human populations are not good models for certain human diseases because of their genetic resistance. This natural resistance of mice to severe GAS sepsis can be overcome by the use of high infectious doses, less resistant strains, or transgenic mice carrying human HLA class II (Nooh et al., 2007) or human plasminogen (Sun et al., 2004; Cole et al., 2006).

Despite this, successful attempts were made with standard inbred mouse lines, e.g., BALB/c, C3H/HeN, and CBA/J, C57BL/10 (Medina et al., 2001), and DBA/2 and 
C57BL/6 (Kansal and Kotb, submitted); whereas these studies were quite informative, conventional mouse models are limited in their genetic pool, and thus one may miss important genotype-trait relations. Our choice was thus to use one of the genetically diversified mouse reference populations generated by the international Complex Trait Consortium (CTC). The CTC strategy, known as the Collaborative Cross (CC), is to construct a very large set of RI strains from a genetically diverse set generated by intercrossing conventional inbred mice (Vogel, 2003; Churchill et al., 2004; Peirce et al., 2004).

RI strains are an ideal resource for systems biology research as they provide a reproducible, highly varied, and diversified yet controlled set of genetic backgrounds for functional genomics, QTL mapping, and identification of interactive gene networks modulating disease phenotype (Valdar et al., 2006). Being inbred, RI strains are perpetually renewable, and have many advantages over $\mathrm{F} 2$ or backcross populations. Experiments performed with RI mice can be repeated in different laboratories and at different times with expected reproducibility. This adds high statistical power. Because the ancestral parents are fully sequenced, the genotyped RI strains afford a powerful tool for identifying genes within QTLs and are particularly useful for mapping multiple and epistatic genetic effects underlying complex phenotypes, including those seen in infectious diseases (Caron et al., 2002; Churchill et al., 2004; Peirce et al., 2004; Ouburg et al., 2005; Roy et al., 2006).

Among the different RI sets of mice, we found the BXD panel more suitable for us than other series (e.g., the AXB set) as the BXD series is much larger, almost 3-times more than used in any other series of experiments and because both parental strains (C57BL/6J and DBA/2J) have been fully sequenced (Waterston et al., 2002), and have been shown to differ in their response to many pathogens (Hormaeche, 1979b, a; Mitsos et al., 2000; Boyartchuk et al., 2001; Boyartchuk and Dietrich, 2002; Mitsos et al., 2003), including GAS (Kansal and Kotb, personal communication).

\section{The Complexity of the Phenotype}

Susceptibility to a particular bacterial infection and disease severity are complex phenotypes likely to be contributed by more than one genetic factor, and by networks and pathways modulated by gene products. Our attempt to attribute one or two sets of genetic loci that predispose mice to or protect them from certain infections could be misleading if we fail to take the complexity of the phenotype into consideration. In this study, we explored the response of a panel of 20 BXD RI mice (plus the two parental strains) to infection with the same dose of GAS and monitored them over a week post-infection. To quantify the animals' susceptibility to GAS and the severity of sepsis, we measured three variables: animal survival, bacteremia, and dissemination in systemic organs (spleen, liver, and lung). Our results provide solid evidence of the complexity of our phenotype. One manifestation of this complexity is that BXD strains exhibited a much wider range of variability in survival (Figure 2-3) and bacteremia (Figure 2-4-A) than the parental strains; yet, the two ancestral strains were on the two extreme sides of the panel of bacterial dissemination in spleen (Figure 2-4-B). This behavior is likely a result of the 
random assortment of many polymorphic loci that accentuate resistance or susceptibility to infection depending on the pattern by which they are inherited from the parental strains to the offspring. Another consequence of the trait complexity is that the different strains did not show identical patterns for the different measured variables (Figures 2-3 and 2-4). There was a significant correlation between survival and bacteremia (Figure 2-1-C), but it only explained $25 \%$ of the variance. Differences in the kinetics of bacterial dissemination to organs of BXD strains in the first $60 \mathrm{~h}$ of disease (Figure 2-5 and Table 2-3) highlights the uniqueness of each strain, and shows the importance of following the course of infection at multiple time points.

Our analysis indicates that bacteremia and dissemination to organs were major predictors of disease severity and mortality; however, it is likely that other factors contribute to mortality including severe toxicity and inflammatory responses in blood and organs.

\section{The Power of Statistical Analysis}

A major part of our study was dedicated to the optimization and standardization of this novel mouse model. We conducted preliminary experiments to optimize the inoculum size and volume, the number of mice to be used per strain, and the best randomization scheme for injecting mice and recording the data. Subsequently we generated a large data set from 445 mice, and we used this data set to estimate the effect of independent confounding factors like sex, age, body weight, and inoculum size (Figure 2-2) as well as the combined effects of these covariates (Table 2-2). As a result of these analyses, we filtered our data set, bringing the number of analyzed cases to 307 , after excluding animals from strains that were tested only once, and those that did not fall in the age range where age and weight were colinear. The analysis of covariates also helped us to estimate the contribution of each factor in predicting susceptibility to the severe form of GAS sepsis therein allowing us to correct the measurable traits (SI, BI, and Log $\mathrm{CFU} /$ spleen) for significant covariates. Correction for age, for example, was a vital alternative to age-matching all animals, which is not feasible because the breeding scheme of 20 strains cannot be synchronized practically. The ability to use a large number of strains and to repeat experiments using the same strains added power to the statistical analysis. Combining the genetic diversity of the RI strains with statistical analysis allowed the dissection of many factors contributing to infection phenotype using relatively small numbers of animals and a large number of strains.

\section{The Preliminary Quantitative Trait Loci Mapping Results}

It is rather risky to attempt to identify QTLs using only $20 \mathrm{BXD}$ strains, and this was not the intention of this study, which focused on developing the model and identifying significant covariates affecting the severity of GAS sepsis. Nonetheless, we were able to exclude plausible regions as major contributors to GAS sepsis severity. For example, we can comfortably exclude any contribution from the $\mathrm{H} 2$ region on chromosome 17. Although our previous studies showed strong association with HLA 
class II genes (Kotb et al., 2002; Nooh et al., 2007), it was not surprising that the mouse $\mathrm{H} 2$ allelic variation does not contribute to GAS sepsis severity because the mouse $\mathrm{H} 2$ molecules are very poor presenters of GAS superantigens that are pivotal in mediating the pathogenesis of the human disease. The BXD model allows us now to map QTLs and identify additional bacterial virulence strategies involved in the pathogenesis of sepsis. The latter are usually masked by the overwhelming effects of superantigens in human studies and the HLA-transgenic mouse model (Kotb et al., 2002; Nooh et al., 2007). Preliminary mapping studies pointed to two regions on mouse chromosome 2, which harbor many genes of relevance to immune modulation. Ongoing studies using additional strains are expected to confirm and narrow down these QTLs.

\section{Conclusion}

The BXD RI strains afforded a good model for the analysis of traits that contribute to severe GAS sepsis. The genetic diversity of these strains exaggerates or suppresses traits seen in the parental strains. Having standardized the model and set major criteria for selecting the animals to be used and analyzed, we will continue generating data by using more BXD strains and bioinformatics tools to map QTLs and determine the genetic basis for the large differences in disease susceptibility among these animals. Mapping QTLs is likely to identify gene variants (polymorphisms, SNPs, or mutations) that underlie the remarkable individual differences that are a hallmark of host-GAS interactions; however, our long-term goal is to generate a disease roadmap by revealing the networks involved in these inter-strain differences.

\section{Materials and Methods}

\section{Mice}

The BXD strains are completely inbred strains generated by repeated mating between siblings starting from F2 intercross progeny between C57BL/6J and DBA/2J (Taylor, 1978; Taylor et al., 1999; Williams et al., 2001; Peirce et al., 2004). In this study, we used 717 mice belonging to a set of $33 \mathrm{BXD}$ strains along with the parental strains, C57BL/6J (B6) and DBA/2J (D2). Among these mice, 218 were used in the preliminary experiments for optimization of inoculum size and experimental conditions, 445 were used in the population-based experiments, and 54 were used in the controlled time-course experiments. The animals chosen for final statistical analysis of the population-based experiments $(n=307)$ were those that satisfied all the conditions determined in the study (see Results) and that belonged to a BXD strain that was used in at least two experiments.

The animals were mated and housed in a pathogen-free colony on hardwood chip bedding in microisolator cages at the VA Medical Center and the University of Tennessee Health Science Center, Memphis, TN. They were maintained on a $12 \mathrm{~h} \mathrm{light-}$ dark cycle, in a room kept at $23{ }^{\circ} \mathrm{C}$ with $50-60 \%$ relative humidity and were given tap 
water and sterile irradiated rodent chow (Harlan Teklad, Madison, WI) ad libitum. All protocols were approved by the Institutional Animal Care and use Committees of Veterans Affairs Medical Center and The University of Tennessee.

\section{Bacteria and Culture Media}

We used the clinical isolate, GAS-5448 (Kansal et al., 2000), representative of the clonal M1T1 strain that has globally disseminated since the mid 1980s and was associated with severe and non-severe GAS infections (Chatellier et al., 2000; Aziz et al., 2005). The bacteria were incubated in Todd Hewitt Broth (DIFCO, Detroit, MI) supplied with $1.5 \%$ of yeast extract (DIFCO, Detroit, MI), or THY, for $17 \mathrm{~h}$, washed twice with sterile phosphate-buffered saline solution, PBS (GIBCO, Carlsband, CA), then diluted to the final inoculum size with sterile PBS. The original isolate had been aliquoted in THY stocks containing $15 \%$ glycerol (Sigma, Saint Louis, MO) at $-80^{\circ} \mathrm{C}$. Before each use, the bacteria were spread on blood agar plates (Becton Dickenson, Franklin Lakes, NJ) for colony isolation and sterility checking.

\section{Experimental Design and Infection Scheme}

Before the start of each experiment, we randomized animals to avoid bias in the order of injection, weighing, or sampling. We kept males and females in different cages. Animals were infected via the tail vein injection with a $2 \pm 1 \times 107 \mathrm{CFU} /$ mouse (unless otherwise stated). Twenty-four hours post-infection, blood was sampled for bacteremia: $20 \mathrm{ml}$ was drawn from the tail vein and diluted 10-fold in sterile saline (SS). During the observation period (7 days), animals were weighed twice a day (8:00 a.m., 4:00 p.m.) and checked for survival three times a day (8:00 a.m., 4:00 p.m., and at midnight). We dissected spleens, livers, and lungs from mice that were sacrificed at specified time points, as well as animals that expired prior to sacrifice, and we homogenized these tissues using a rotor stator homogenizer (Omni International, Marietta, GA) in SS, and a sample was used to estimate bacterial loads.

\section{Design of Controlled Time-course Experiments}

Time-course experiments were designed to investigate the kinetics of bacterial dissemination in the early stages of the infection. These experiments were performed following the same procedure as the population-based experiments (see experimental design and infection scheme), except that equal numbers of animals belonging to each strain (typically 3 animals per strain per time point) were sampled for blood, spleen, liver, and lung at $18 \mathrm{~h}, 36 \mathrm{~h}$, and $60 \mathrm{~h}$ post-infection. Regardless of the specified sampling time, all animals were bled through the tail vein $(20-50 \mu 1)$ for determination of bacteremia at $18 \mathrm{~h}$. At each time point, the animals were dissected, and their organs (spleens, livers, and lungs) were homogenized in $10 \mathrm{ml} \mathrm{SS}$ each. 


\section{Bacterial Loads in Blood and Organs}

To determine bacterial loads in blood and organ homogenates, we diluted $10 \mathrm{ml}$ of either sample (tail vein blood or homogenate) serially (up to $10^{-7}$ ) and plated $10 \mathrm{ml}$ of each dilution (as well as the original sample) on the surface of blood agar plates (Becton Dickenson, Franklin Lakes, NJ). After $24 \mathrm{~h}$ incubation, all plates were counted, and the number of colony forming units $(\mathrm{CFU})$ per $\mathrm{ml}$ or per organ was calculated $(\mathrm{CFU} /$ organ $=$ $\mathrm{CFU} / \mathrm{ml}$ homogenate $\mathrm{x} 10)$.

\section{Data Handling and Statistical Analysis}

Results were recorded three times daily (or whenever an animal died). Data were reviewed for accuracy and consistency at the end of every experiment by a different set of investigators. We dedicated a considerable amount of time to data recording, error checking, and filtration after each experiment. Mice lost due to factors unrelated to infection were flagged in the database and excluded from the final analysis. We performed all primary calculations and sorting operations using simple features and functions of Microsoft Excel. Excel files were later exported to Data Desk (version 6.2) software (Data Description, Inc., Ithaca, NY; www.datadesk.com), which we used for all statistical analyses and tests, including clustering, correlation analysis, linear regression, multiple regression, and general linear model (GLM) analysis by ordinary least squares analysis of variance (OLS ANOVA).

\section{Preliminary Quantitative Trait Loci Mapping}

For QTL mapping, we used the web-based complex trait analysis platform, WebQTL (Wang et al., 2003) available on the GeneNetwork web site (http://www.genenetwork.org). WebQTL evaluates potential QTL at regular intervals and estimates the significance at each location with a graphical representation of the likelihood ratio statistics (LRS). 


\title{
CHAPTER 3. AN UNBIASED SYSTEMS GENETICS APPROACH TO MAPPING GENETIC LOCI MODULATING SUSCEPTIBILITY TO SEVERE STREPTOCOCCAL SEPSIS ${ }^{*}$
}

\begin{abstract}
Summary
Striking individual differences in severity of group A streptococcal (GAS) sepsis have been noted, even among patients infected with the same bacterial strain. We had provided evidence that HLA class II allelic variation contributes significantly to differences in systemic disease severity by modulating host responses to streptococcal superantigens. Inasmuch as the bacteria produce additional virulence factors that participate in the pathogenesis of this complex disease, we sought to identify additional gene networks modulating GAS sepsis. Accordingly, we applied a systems genetics approach using a panel of advanced recombinant inbred mice. By analyzing disease phenotypes in the context of mice genotypes we identified a highly significant quantitative trait locus (QTL) on Chromosome 2 between $22-34 \mathrm{Mb}$ that strongly predicts disease severity, accounting for $25-30 \%$ of variance. This QTL harbors several polymorphic genes known to regulate immune responses to bacterial infections. We evaluated candidate genes within this QTL using multiple parameters that included linkage, gene ontology, variation in gene expression, co-citation networks, and biological relevance, and identified interleukin1 alpha and prostaglandin E synthases pathways as key networks involved in modulating GAS sepsis severity. The association of GAS sepsis with multiple pathways underscores the complexity of traits modulating GAS sepsis and provides a powerful approach for analyzing interactive traits affecting outcomes of other infectious diseases.
\end{abstract}

\section{Introduction}

Infectious diseases, like most human diseases, are modulated by complex traits. Susceptibility and clinical outcomes of infections are often a manifestation of interactions between the host's complex traits and the pathogen's virulence components.

Identification of genes and molecular networks that influence host responses to infectious agents can provide a disease road map that would focus the discovery of effective diagnostics and therapeutics.

Group A streptococci (GAS) are classified on the basis of surface M protein antigens into more than 100 serotypes, but recent studies showed a high degree of diversification within a serotype that is driven primarily by horizontal gene transfer (Aziz et al., 2005; Sumby et al., 2005; Walker et al., 2007). It is widely believed that such events are responsible for the emergence of highly virulent strains in the 1980s, including a hypervirulent M1T1 clonal strain, coinciding with the resurgence of severe invasive

* Source: Reprinted with permission. Abdeltawab, N.F., Aziz, R.K., Kansal, R., Rowe, S.L., Su, Y., Gardner, L., Brannen, C., et al. (2008) An unbiased systems genetics approach to mapping genetic loci modulating susceptibility to severe streptococcal sepsis. PLoS Pathog 4: e1000042. 
GAS infections associated with streptococcal toxic shock syndrome (STSS) and necrotizing fasciitis (NF), also known as the "flesh eating" disease (Low et al., 1997). We showed that this hypervirulent M1T1 clinical strain can cause sore throat or mild bacteremia and erysipelas in some patients, while in others it can cause STSS and NF (Chatellier et al., 2000). These findings suggested a strong role for host factors in modulating the outcome of infection by this highly virulent strain. Indeed, we found that allelic variations in host HLA class II haplotypes are associated with markedly different outcomes of GAS sepsis in humans (Kotb et al., 2002; Kotb et al., 2003). We confirmed these associations using HLA transgenic mice that were expressing different HLA class II alleles (Nooh et al., 2007). Inasmuch as STSS pathogenesis is largely mediated by streptococcal superantigens (Strep SAgs), and because HLA class II molecules serve as SAg receptors, such an association was expected. However, GAS is rich in many other virulence factors, and we believe that some of those virulence factors, beside SAgs, may also interact with additional host factors to modulate host responses to GAS infections.

To identify additional host factors involved in GAS severity we needed an approach that allowed us to uncover the effect of interactions between complex, polymorphic genetic traits that may modulate sepsis outcomes. Previous animal models for GAS sepsis included various regular conventional inbred mouse strains (Medina et al., 2001; Goldmann et al., 2004b), congenic strains (Goldmann et al., 2005a), and HLA transgenic mice (Welcher et al., 2002; Nooh et al., 2007). These models confirmed that host genetic variability can have a strong effect on infection outcome. Despite their significant utility, these mouse models do not offer the genetic diversity that is characteristic of the human population.

In this study, we used a panel of advanced recombinant inbred (ARI) mice as a genetically diverse, segregating reference population that affords a powerful tool for systems genetics approaches. Recombinant inbred (RI) strains have been successfully used to map quantitative trait loci (QTLs) associated with various phenotypes and diseases (Hardy et al., 2001; Rosen and Williams, 2001; Grizzle et al., 2002; Peirce et al., 2003; Chesler et al., 2005; Mountz et al., 2005; Aziz et al., 2007; Miyairi et al., 2007). We used the BXD panel of ARI mice derived from C57BL/6J (B6) and DBA/2J (D2) strains and consisting of homozygous, inbred BXD lines, each of which is genetically distinct. Using this genetically diverse BXD panel, we mapped QTLs modulating GAS sepsis severity, and identified candidate genes within these QTLs that were parsed into pathways likely to modulate the severity of this complex infectious disease.

\section{Results}

\section{Variable Susceptibility to Severe GAS Sepsis in Genetically Distinct Mice}

Our initial studies (Aziz et al., 2007) demonstrated that there is considerable variability among BXD strains with respect to susceptibility to severe GAS sepsis. To quantify differences among strains in this study, we used three main quantitative traits, namely animal survival, bacteremia, and bacterial dissemination to spleen. 
We infected mice ( $\mathrm{n}=5-26$ mice per strain), belonging to 32 strains (30 different BXD strains and their parental strains, B6 and D2), intravenously (i.v.) with the same bacterial dose $\left(2 \pm 1.5 \times 10^{7} \mathrm{CFU} /\right.$ mouse $)$. Survival was expressed as normalized corrected relative survival indices (cRSI) calculated for each of the 32 strains as detailed in materials and methods. We observed significant difference in relative susceptibility to GAS sepsis across the BXD panel ( $\mathrm{P} \leq 0.0001)$, Figure $3-1-\mathrm{A}$. This wide range of susceptibility across the various strains, together with the finding that some of the strains showed phenotypes considerably more exaggerated than the parental strains (B6 and D2), is an illustration of how different combination of polymorphic genes can manifest quite differently according to the overall genetic context.

Similarly, we determined bacterial load in blood (log CFU/ml blood), 24 h postinfection and found considerable variation across the strains. In general there was an inverse correlation between mice survival and extent of bacteremia $(r=-0.471, \mathrm{P} \leq$ $0.0001, \mathrm{R}^{2}=22.2 \%$ ), Figure $3-1-\mathrm{B}$. Bacterial dissemination to spleen also varied across the strains, showing a stronger inverse correlation with mice survival $(\mathrm{r}=-0.717, \mathrm{P} \leq$ $0.0001, \mathrm{R}^{2}=51.4 \%$ ), Figure 3-1-C.

Although these inverse correlations between mice survival and bacterial load in blood and spleen made biological sense because it is anticipated that susceptible strains would have higher bacterial load than in resistant strains, there were several exceptions. For example, although strains BXD44 and BXD45 are ranked susceptible based on their survival, they showed low bacterial load in blood and spleen. Similarly, BXD43 and BXD85 strains, which are ranked resistant, had a high bacterial load in their spleen. Another interesting observation was that, in general, susceptible strains survival was better correlated with bacterial load in blood and spleen than resistant strains. Together, these findings confirm that there is more than one mechanism modulating differential susceptibility or resistance to severe GAS sepsis (Aziz et al., 2007).

\section{Genome-wide Scans for Mapping GAS Susceptibility Quantitative Trait Loci}

We used bioinformatics tools available through Gene Network (GN) to link measured phenotypes to strains genotypes. Each of the quantified phenotypes was analyzed in the context of the studied mice genotypes and single nucleotide polymorphism (SNPs) using 3795 SNPs and microsatellite markers for BXD strains. Significant QTLs modulating survival, bacteremia $24 \mathrm{~h}$ post-infection and bacterial dissemination to spleen mapped to mouse Chromosome (Chr) 2.

The strongest QTL modulating mouse survival (cRSI) mapped to mouse Chr 2 between 22-34 Mb, with an likelihood ratio statistic (LRS) of $34.2(\mathrm{P} \leq 0.0000001)$, Figure 3-2-A. A second less significant QTL was also mapped on the same chromosome between $125-150 \mathrm{Mb}$ with an LRS of $12(\mathrm{P} \leq 0.001)$, and a third QTL on Chr X, Figure 3-2-A. The QTLs for bacteremia and bacterial dissemination to spleen overlapped with 
Figure 3-1. Differential susceptibility to GAS sepsis among different BXD strains and their parental strains.
(A) Rank-ordered bar chart of survival expressed as mean values of coefficient of mean corrected relative survival indices (cRSI) across 30 different BXD strains and their parental strains (DBA/2J and $\mathrm{C} 57 \mathrm{Bl} / 6 \mathrm{~J})$. $\mathrm{N}=489$ belonging to 32 strains; number of mice used per strain is indicated.(B) Bar chart of GAS bacteremia expressed as coefficient of mean values of corrected log blood CFUs 24 h post injection (corrected log $\mathrm{CFU} / \mathrm{ml}$ blood). (C) Bar chart of GAS dissemination to spleen expressed as coefficient of corrected bacterial load in spleen (log CFU/spleen). B and C Strains are ordered by their corrected relative survival indices with the parental strains at the two extremes of the X- axis. Error bars represent the standard errors of the means. Statistical test is two-way ANOVA (with correction to covariates) $\mathrm{P} \leq 0.0001$. 


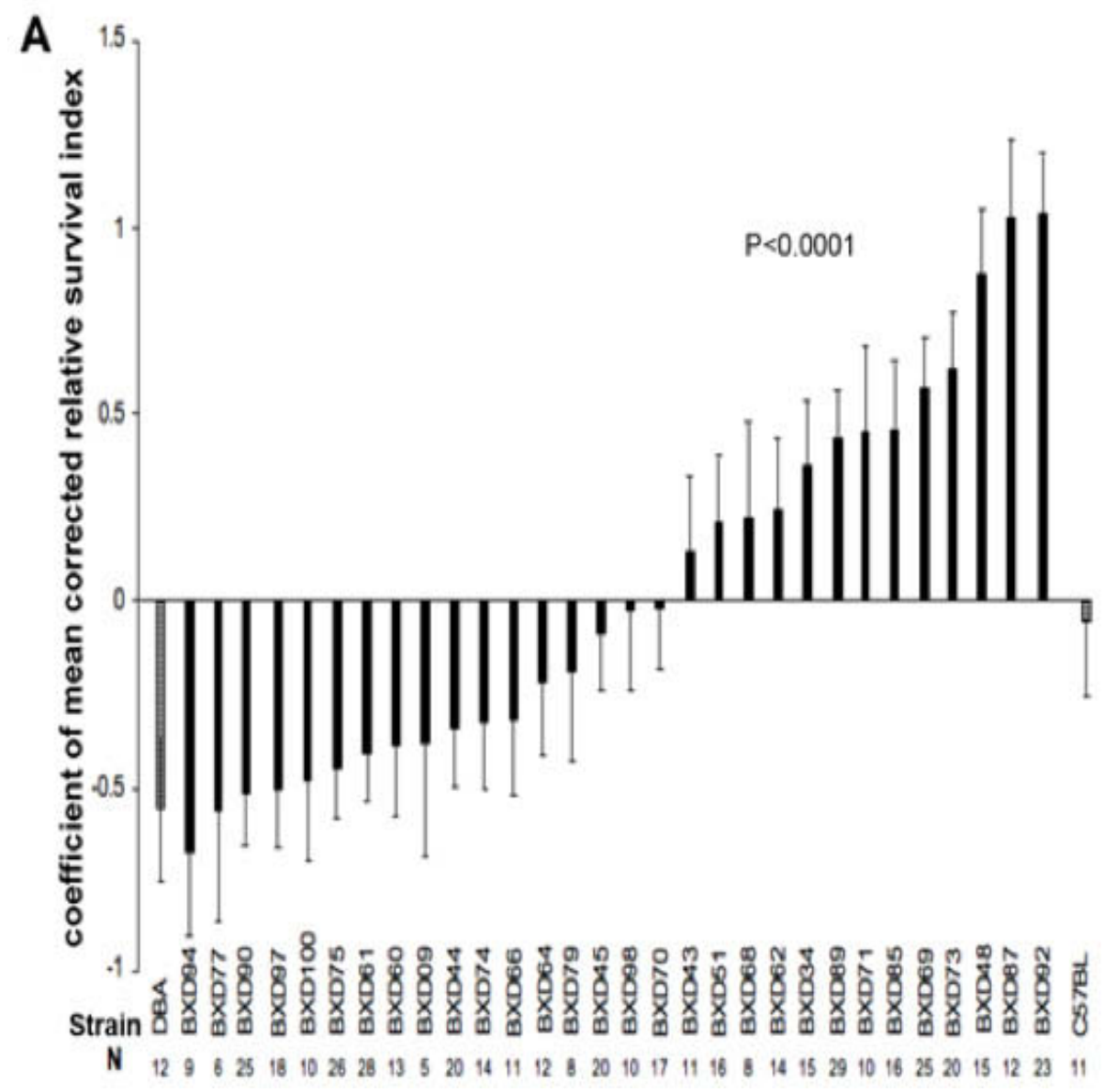



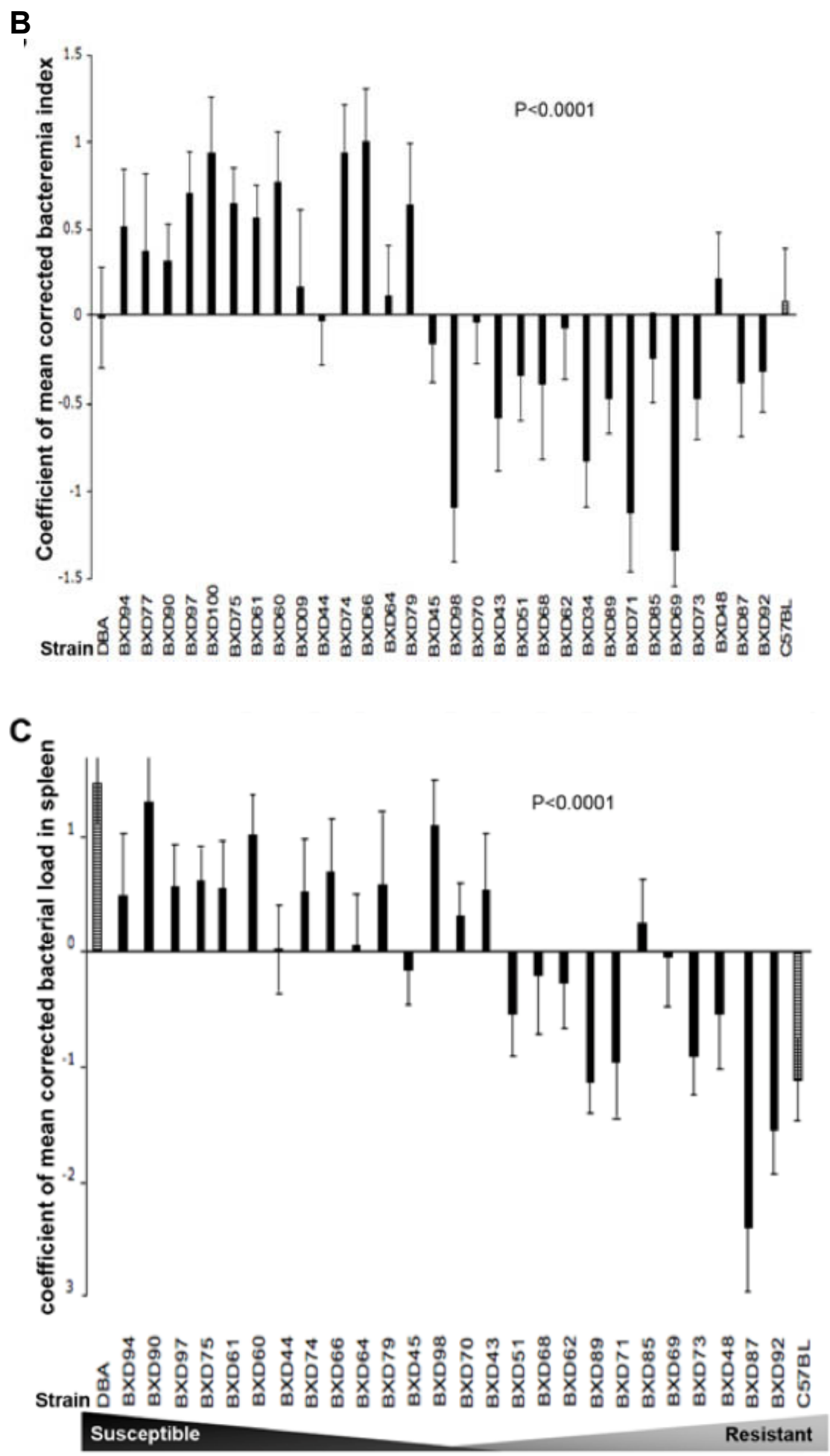

Figure 3-1. (Continued). 
Figure 3-2. Genome-wide scan for mice susceptibility to GAS sepsis showing mapped QTL on Chr 2.

Interval mapping of survival (expressed as corrected relative survival index, cRSI), showing a significant QTL (based on 1000 permutation tests) on Chr 2 between 22-34 $\mathrm{Mb}$ with LRS of $34.2(\mathrm{P} \leq 0.0000001)$, and a suggestive QTL between $125-150 \mathrm{Mb}$ with LRS of 12 (P $\leq 0.001)$. (B) Whole genome interval mapping of GAS bacteremia using bacterial load $24 \mathrm{~h}$ post injection expressed as corrected $\log \mathrm{CFU} / \mathrm{ml}$ blood, showing two QTLs on Chr 2: first QTL between 22-34 Mb with LRS of $24.5(\mathrm{P} \leq 0.00001)$ and a second with LRS of $17(\mathrm{P} \leq 0.0001)$ between $125-150 \mathrm{Mb}$. (C) Whole genome mapping of GAS sepsis using tissue dissemination of GAS expressed as corrected log CFU/spleen with QTL between $125-150 \mathrm{Mb}$ with LRS of 15 (P $\leq 0.0001)$. Red line indicates significant LRS, while grey line indicates suggestive LRS. Upper $\mathrm{x}$-axis shows mouse chromosomes, and lower $\mathrm{x}$-axis shows physical map in mega bases, $\mathrm{y}$-axis represents linkage in LRS score. 

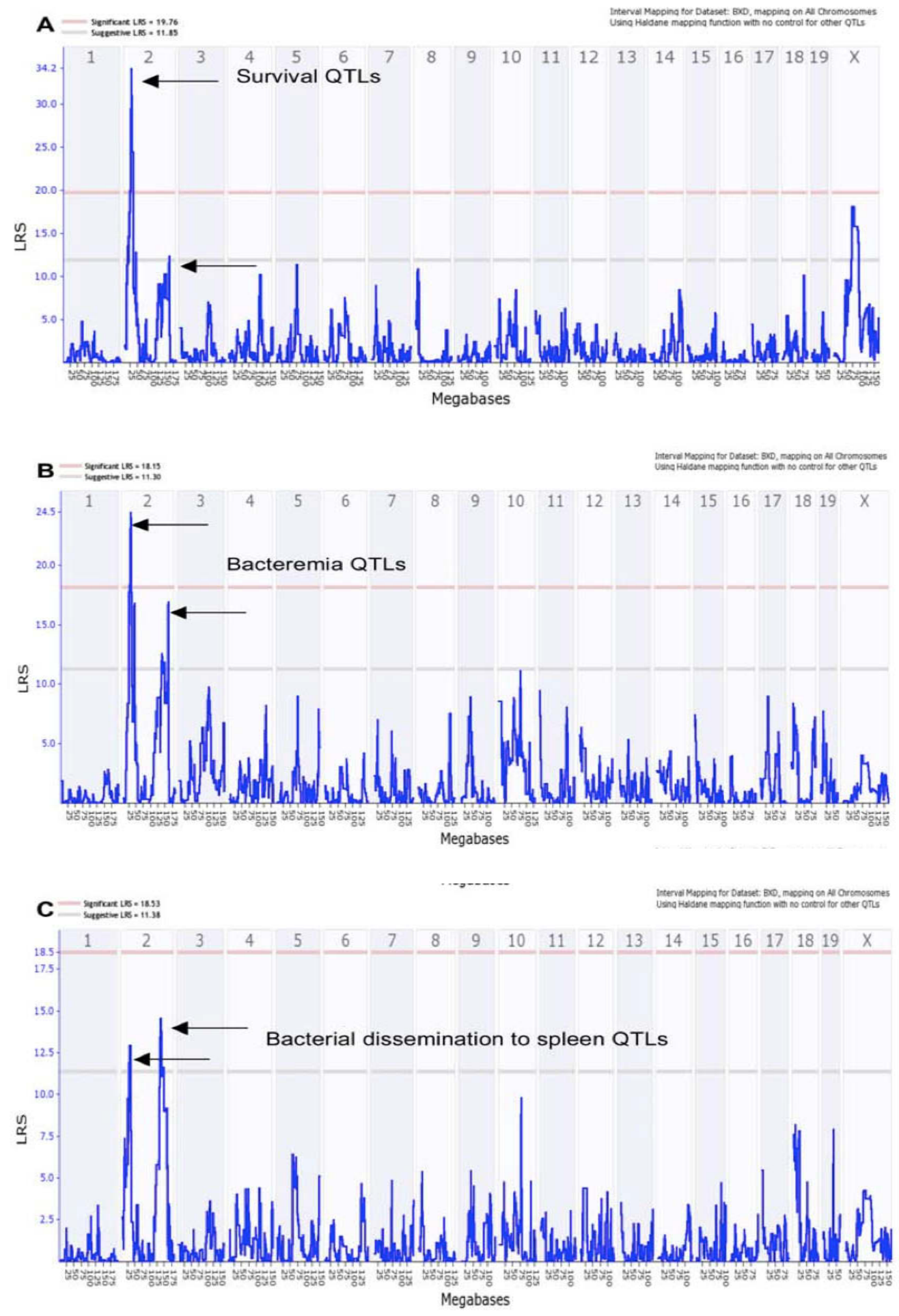
those for survival, with slight difference in significance. The first QTL modulating bacteremia mapped to Chr 2 between $22-34 \mathrm{Mb}$ with an LRS of 24.5 ( $\mathrm{P} \leq 0.00001)$, Figure 3-2-B. The second QTL mapped to the same chromosome between $125-150 \mathrm{Mb}$ with an LRS of 17 (P $\leq 0.0001)$, Figure 3-2-B. A QTL modulating bacterial dissemination to spleen also mapped to $\mathrm{Chr} 2$ between $125-150 \mathrm{Mb}$ with LRS of 15 (P $\leq$ 0.001), Figure 3-2-C.

To further investigate and narrow down the mapped region on $\mathrm{Chr} 2$, we resorted to haplotype maps of BXD strains to select additional strains that may be informative in validating, and further confirming mapped QTLs.

\section{In silico Prediction of BXD Susceptibility to Severe GAS Sepsis}

Our mapped QTLs directed us to select more strains for survival experiments based on differences in their haplotypes within the QTLs. For example, we selected BXD100 with a $D$ haplotype at Chr 2 between 22-34 Mb region, Figure 3-3-A, and found it to be susceptible. Similarly, we tested BXD87 with a $B$ haplotype in the same region and this strain as predicted, exhibited a resistant phenotype. To further narrow down the mapped QTLs, we chose strains with breakage in the mapped interval, i.e. with recombination at $\mathrm{Chr} 2$ region 22-34 $\mathrm{Mb}$ for further survival testing (Figure 3-3-A). BXD34 with a $B$ haplotype at $\mathrm{Chr} 2$ between 24-33.15 Mb region was resistant, while BXD60 with a $B$ haplotype at $\mathrm{Chr} 2$ between 20-23.27 Mb region was susceptible, Figure 3-1-A and 3-3-A. This narrowed down the relevant susceptibility region to Chr 2 between 23.27-33.15 Mb. Using more strains with recombination at this narrowed region, we found BXD51, 64, and 79 to be quite interesting as they showed intermediate resistance suggesting that genes at this region are candidate modulators of the mapped QTL, Figure 3-3-B. Another interesting strain was BXD94 that we found susceptible to infection, yet has a heterozygous genotyping, suggesting that $D$ allele exhibited dominance, Figure 3-3-B.

Similarly, we inspected haplotypes of studied BXD strains at the second QTL between $125-150 \mathrm{Mb}$ (Figure 3-3-C), the majority of susceptible strains had $D$ alleles, while resistant strains had $B$ alleles with some exceptions, for example BXD77 is a susceptible strain, yet had $B$ alleles. It was interesting to find that the susceptible strain BXD44 had $B$ alleles at this QTL (Figure 3-3-C), suggesting that observed lower bacterial load in blood compared to other susceptible strains (Figure 3-1-B) might be modulated by $B$ allele in the second QTL. Meanwhile, BXD48, a resistant strain, has a breakage at $135.9 \mathrm{Mb}$ (Figure 3-3-C) with $D$ alleles suggesting that its relatively higher bacterial load in blood (Figure 3-1-B) might be modulated by the second QTL. Collectively, these findings suggest that both loci modulate different observed phenotypes of GAS sepsis severity. 
Figure 3-3. Recombinant inbred BXD strain distribution patterns at region of interest on Chr 2.

(A) Haplotype maps of the first QTL on Chr 2 between 20-34 Mb showing available BXD strains. Haplotype maps were used for in silico selection of strains for survival studies based on the strain distribution patterns, where B allele (blue bars) inherited from the resistant parent $\mathrm{C} 57 \mathrm{Bl} / 6 \mathrm{~J}$ and $\mathrm{D}$ alleles form the susceptible parent $\mathrm{DBA} / 2 \mathrm{~J}$, beige bars indicate strains that are heterozygous at this region, resembling an F1. Arrows indicate BXD strains 34, 51, 60, 64, 79, 94, and 100. Recombinant inbred BXD strain distribution patterns at region of interest on Chr 2. (B) Haplotype maps of the first QTL region on $\mathrm{Chr} 2$ between $20-34 \mathrm{Mb}$ across $\mathrm{BXD}$ strains used in the population-based survival experiments showing the pattern of alleles inherited from each parent at region of interest on Chr 2. The different BXD strains are rank-ordered according to their susceptibility to GAS sepsis from susceptible to more resistant. Resistant strains show a pattern of accumulation of alleles inherited from resistant parent (B) C57Bl/6 (blue bars) while the susceptible strains show a pattern of alleles from susceptible parent (D) DBA/2J (red bars), with the exception of BXD94 strain, heterozygous (beige bars) at the QTL region. (C) Haplotype maps of the second QTL region on Chr 2 between $125-150$ $\mathrm{Mb}$ across BXD strains used in the population-based survival experiments showing the pattern of alleles inherited from each parent at region of interest on $\mathrm{Chr} 2$. The different BXD strains are rank-ordered according to their susceptibility to GAS sepsis from susceptible to more resistant. BXD genotype data set can be downloaded from www.genenetwork.org/genotypes/BXD.geno. 


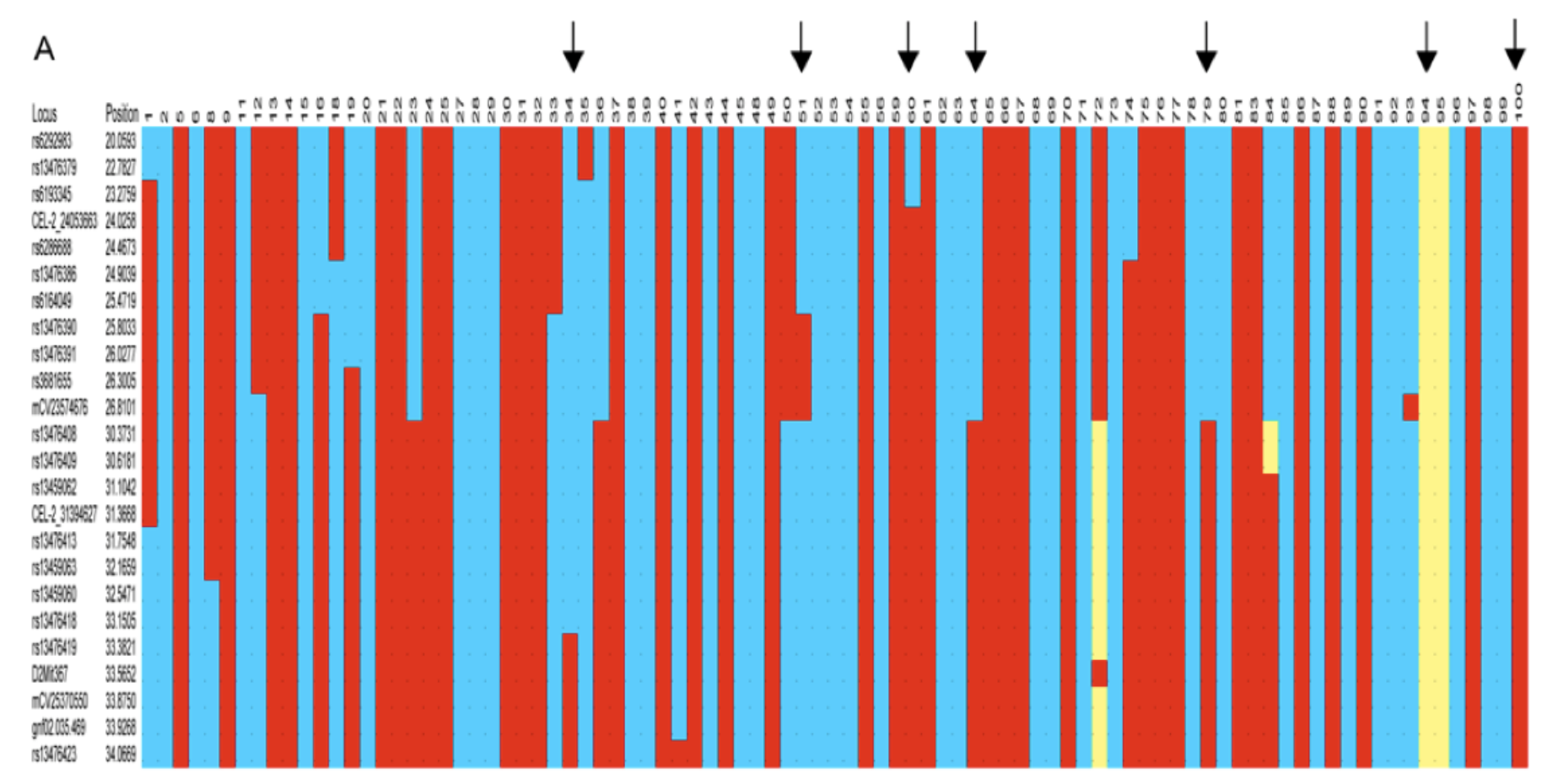



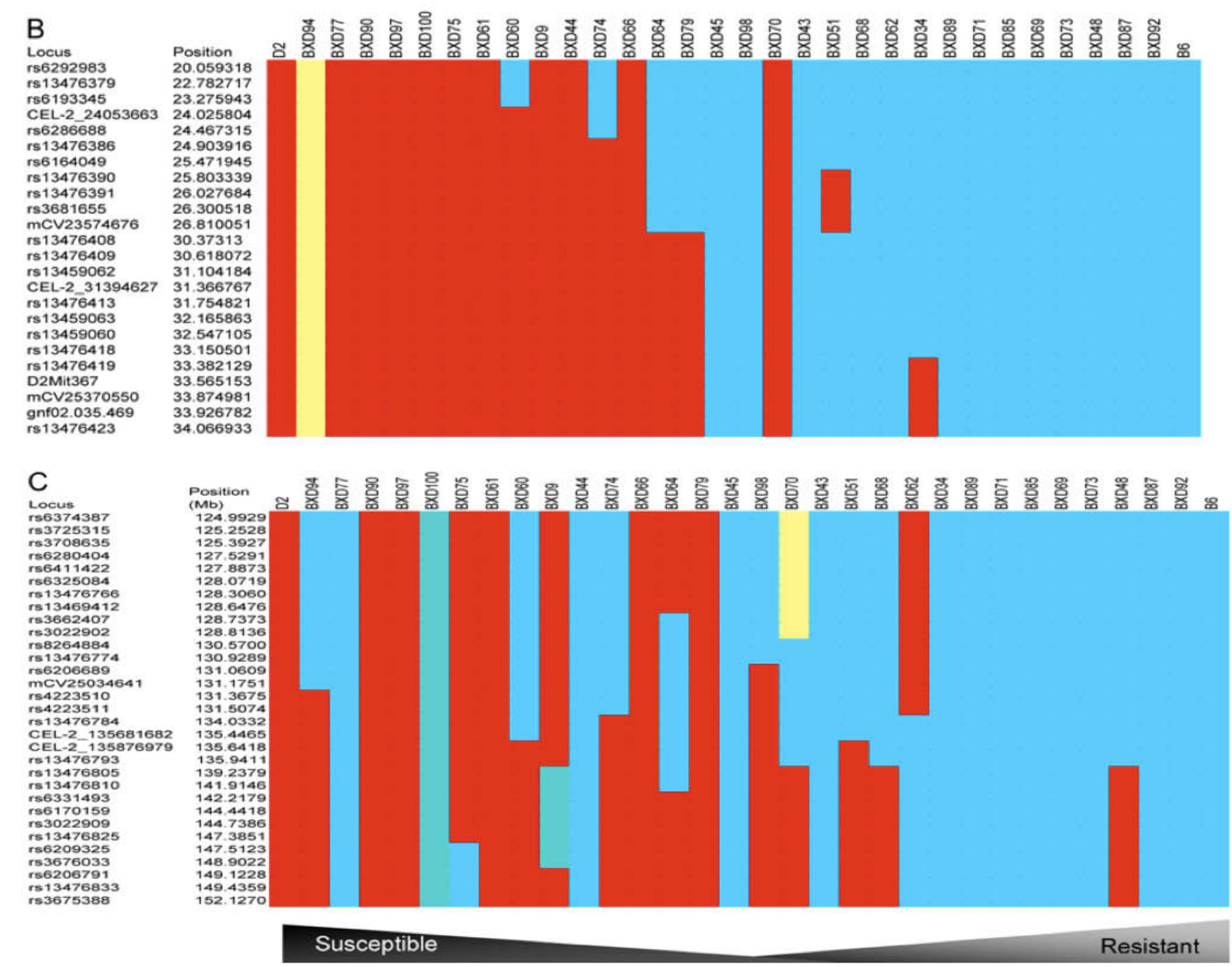

Figure 3-3. (Continued). 


\section{Mining for Candidate Genes and Pathways in Mapped Loci}

Based on our in silico strain selection and QTL validation by experimental assessment, we evaluated candidate genes within the mapped QTLs, taking into accountlinkage, gene ontology analyses, co-citation networks, and biological relevance. To categorize genes and transcripts in the mapped QTLs into functional pathways, we performed functional analyses using Ingenuity Pathways Analysis (IPA) (www.ingenuity.com). We parsed our genes into 50 functional networks; the most relevant networks involved those associated with immune response, cell signaling, cellular assembly and organization, and lipid metabolism. From these interrelated pathways, we selected 37 representative genes to study their role in GAS susceptibility QTL by quantitative analysis of their expression levels in selected resistant and susceptible strains (groups) pre- and post-infection (Appendix A. Table A-1).

We found that 28 out of a representative 37 genes were differentially expressed post- infection (Appendix A. Table A-2), of those 14 genes were down regulated in the resistant group while up regulated in the susceptible group post-infection. Nine genes were down regulated in both groups post-infection, and five were up regulated in both groups post-infection (Appendix A. Table A-2). Fourteen of the 28 differentially expressed genes showed significant change post-infection in both resistant and susceptible strains (Table 3-1 and Figure 3-4). In general, susceptible strains showed an increase in the relative expression levels post-infection (19 genes) of both pro and antiinflammatory genes, e.g. interleukin 1 alpha (Ill $\alpha)$, and Il1 receptor antagonist (Illrn) respectively. By contrast, in the resistant BXD strains, most of the tested genes showed a decrease ( 23 genes) in expression levels post-infection with few exceptions (five genes) e.g. TNF receptor associated factor 1 (Traf1) (Figure 3-4 and Appendix A. Table A-2). Differentially expressed genes were associated with both innate and early adaptive immune response. Among those associated with early immune response, Illa, Illrn, prostaglandin E synthase (Ptges), and Ptges 2 were up regulated in susceptible strains, whereas their levels decreased in resistant strains. Several of the differentially expressed genes show polymorphisms as SNPs between the parental strains B6 and D2, suggesting that these polymorphic genes modulate differential response to infection (Appendix C). The differential expression of IL1 $\alpha$ was confirmed at the protein level (data not shown).

We parsed the differentially expressed genes into pathways, using IPA, IL1 and prostaglandins were key early response molecules modulating susceptibility to severe GAS sepsis in two of the mapped networks, which are shown merged in Figure 3-5. The first network $\left(\mathrm{P}<10^{-27}\right)$ comprised of genes related to lipid metabolism and innate immunity, e.g. Il1a, Il1rn, Ptges, while the second network $(\mathrm{P}<0.01)$ contained genes modulating nucleic acid metabolism, energy production and host responses to injury e.g. Ectonucleoside triphosphate diphosphohydrolase 2 (Entpd2) (Figure 3-5).

\section{Discussion}

It has been established that networks of multiple pathways, rather than a single gene, modulate traits and affect susceptibility and outcomes of virtually all diseases. The 
Table 3-1. The relative expression levels of candidate genes post infection in resistant and susceptible strains expressed as ratio of post/pre infection in susceptible and resistant strains.

\begin{tabular}{|c|c|c|c|c|c|}
\hline Gene ID & Gene & $\begin{array}{l}\text { Resistant } \\
\text { Strains }\end{array}$ & P-value ${ }^{a}$ & $\begin{array}{l}\text { Susceptible } \\
\text { Strains }\end{array}$ & P-value \\
\hline \multicolumn{6}{|c|}{$\begin{array}{l}\text { Genes down regulated in resistant group while up regulated } \\
\text { in susceptible group }\end{array}$} \\
\hline Anapc2 & Anaphase promoting complex subunit 2 & 0.551 & $0.022 *$ & 1.475 & 0.509 \\
\hline Ill $\alpha$ & Interleukin $1 \mathrm{a}$ & 0.739 & $0.010 *$ & 44.525 & $0.062 \dagger$ \\
\hline Illrn & Interleukin 1 receptor antagonist & 0.851 & 0.680 & 54.687 & 0.221 \\
\hline Ptges & Prostaglandin E synthase & 0.417 & $0.001 *$ & 6.363 & $0.028 *$ \\
\hline Ptges2 & Prostaglandin E synthase 2 & 0.913 & 0.774 & 1.456 & $0.005 *$ \\
\hline $\operatorname{Sh} 2 d 3 c$ & $\mathrm{SH} 2$ domain containing $3 \mathrm{C}$ & 0.162 & $0.055 \dagger$ & 1.009 & 0.980 \\
\hline \multicolumn{6}{|c|}{$\begin{array}{l}\text { Genes down regulated in both resistant group and } \\
\text { susceptible groups }\end{array}$} \\
\hline Entpd2 & Ectonucleoside triphosphate diphosphohydrolase 2 & 0.359 & $0.010 *$ & 0.654 & $0.064 \dagger$ \\
\hline Edf1 & Endothelial differentiation-related factor 1 & 0.349 & $0.095 \dagger$ & 0.470 & $0.014 *$ \\
\hline Garnl3 & GTPase activating RANGAP domain-like 3 & 0.601 & 0.142 & 0.674 & $0.070 \dagger$ \\
\hline Nfatc2 & $\begin{array}{l}\text { Nuclear factor of activated t-cells, cytoplasmic, } \\
\text { calcineurin-dependent } 2\end{array}$ & 0.178 & $0.095 \dagger$ & 0.693 & 0.208 \\
\hline Phpt1 & Phosphohistidine phosphatase 1 & 0.229 & $0.077 \dagger$ & 0.714 & 0.233 \\
\hline Psmd5 & $\begin{array}{l}\text { Proteasome (prosome, macropain) 26S subunit, } \\
\text { non-ATPase } 5\end{array}$ & 0.644 & $0.006 *$ & 0.963 & 0.652 \\
\hline Ppp2r4 & Protein phosphatase $2 \mathrm{~A}$ regulatory subunit $\mathrm{B}$ & 0.902 & 0.256 & 0.734 & $0.091 \dagger$ \\
\hline \multicolumn{6}{|c|}{$\begin{array}{l}\text { Genes up regulated in both resistant group and } \\
\text { susceptible group }\end{array}$} \\
\hline Trafl & TNF receptor associated factor 1 & 1.826 & 0.300 & 5.995 & $0.066 \dagger$ \\
\hline
\end{tabular}

\footnotetext{
${ }^{a}$ Significance based on t-test, $* \mathrm{P}<0.05, \uparrow \mathrm{P}=0.06-0.08$
} 


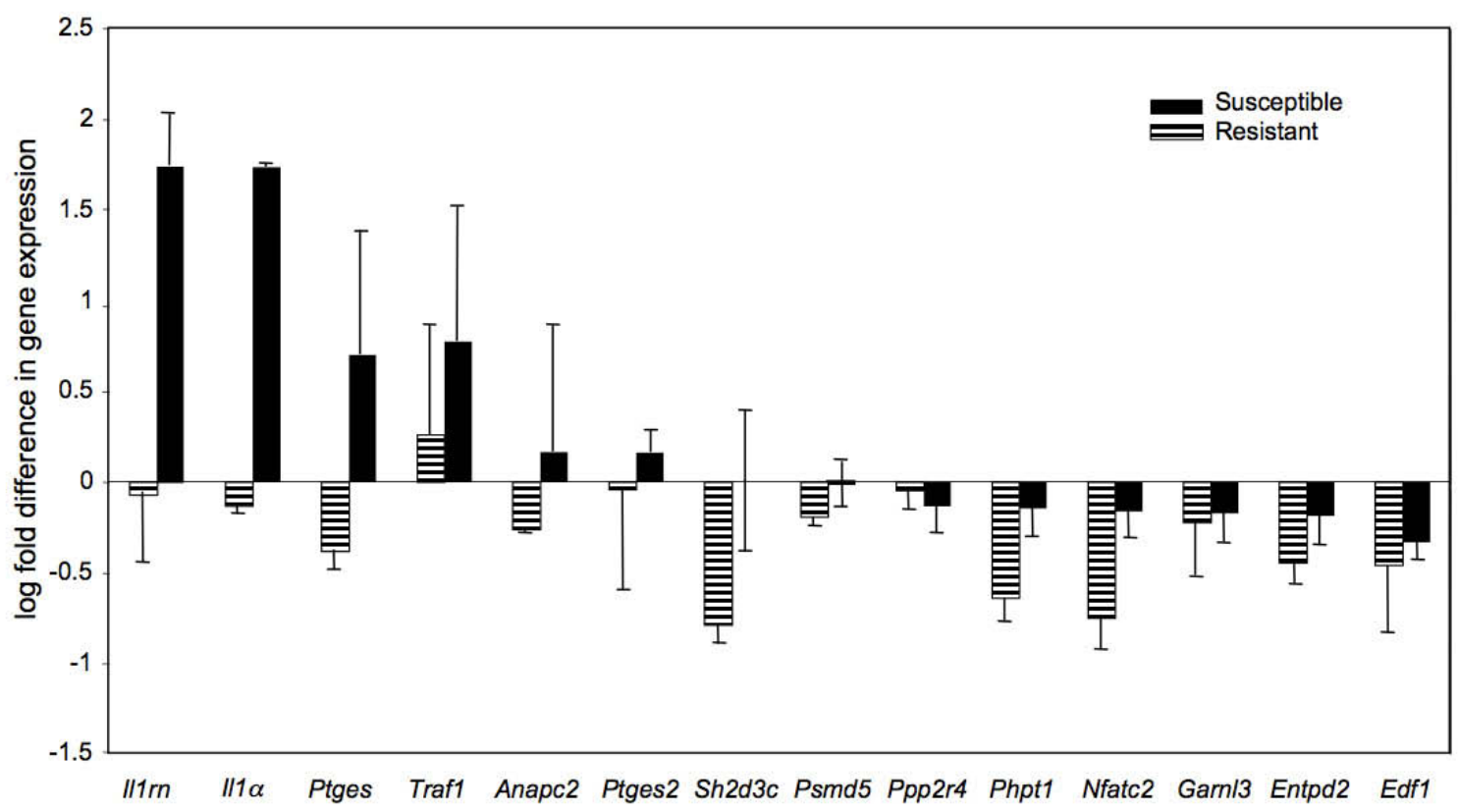

Figure 3-4. Patterns of differential gene expression levels of candidate genes post infection in susceptible and resistant strains.

Quantitative PCR results showing levels of gene expression of 14 genes with significant $(\mathrm{P}=0.05-0.08)$ change post-infection in susceptible and resistant strains, expressed as $\log _{10}$ fold differences in gene expression level post-infection. Genes are grouped to three groups, a) genes up regulated in susceptible strains post-infection and decreased in resistant strains post-infection, b) genes down regulated in both susceptible and resistant strains post-infection, and c) genes up regulated in both susceptible and resistant strains post-infection. Susceptible strains are represented as solid black bars, we used two highly susceptible strains BXD61 and BXD90, and resistant strains as dashed bars, we used two highly resistant strains BXD 73 and BXD87. The bars represent 2-4 biological replicates run in three technical replicates each and significance is based on t-test. Anapc2, anaphase promoting complex subunit 2; Entpd2, ectonucleoside triphosphate diphosphohydrolase 2; Edf1, endothelial differentiation-related factor 1; Garnl3, GTPase activating RANGAP domain-like 3; Il1a, interleukin 1 alpha; Il1rn, interleukin 1 receptor antagonist; Nfatc2, nuclear factor of activated t-cells, cytoplasmic, calcineurin-dependent 2; Phpt1, phosphohistidine phosphatase 1; Ptges, prostaglandin E synthase; Ptges2, prostaglandin E synthase 2; Psmd5, proteasome (prosome, macropain) 26S subunit, non-ATPase 5; Ppp2r4, Protein phosphatase $2 \mathrm{~A}$ regulatory subunit B; Sh2d3c, SH2 domain containing 3C; Traf1, TNF alpha receptor associated factor. 


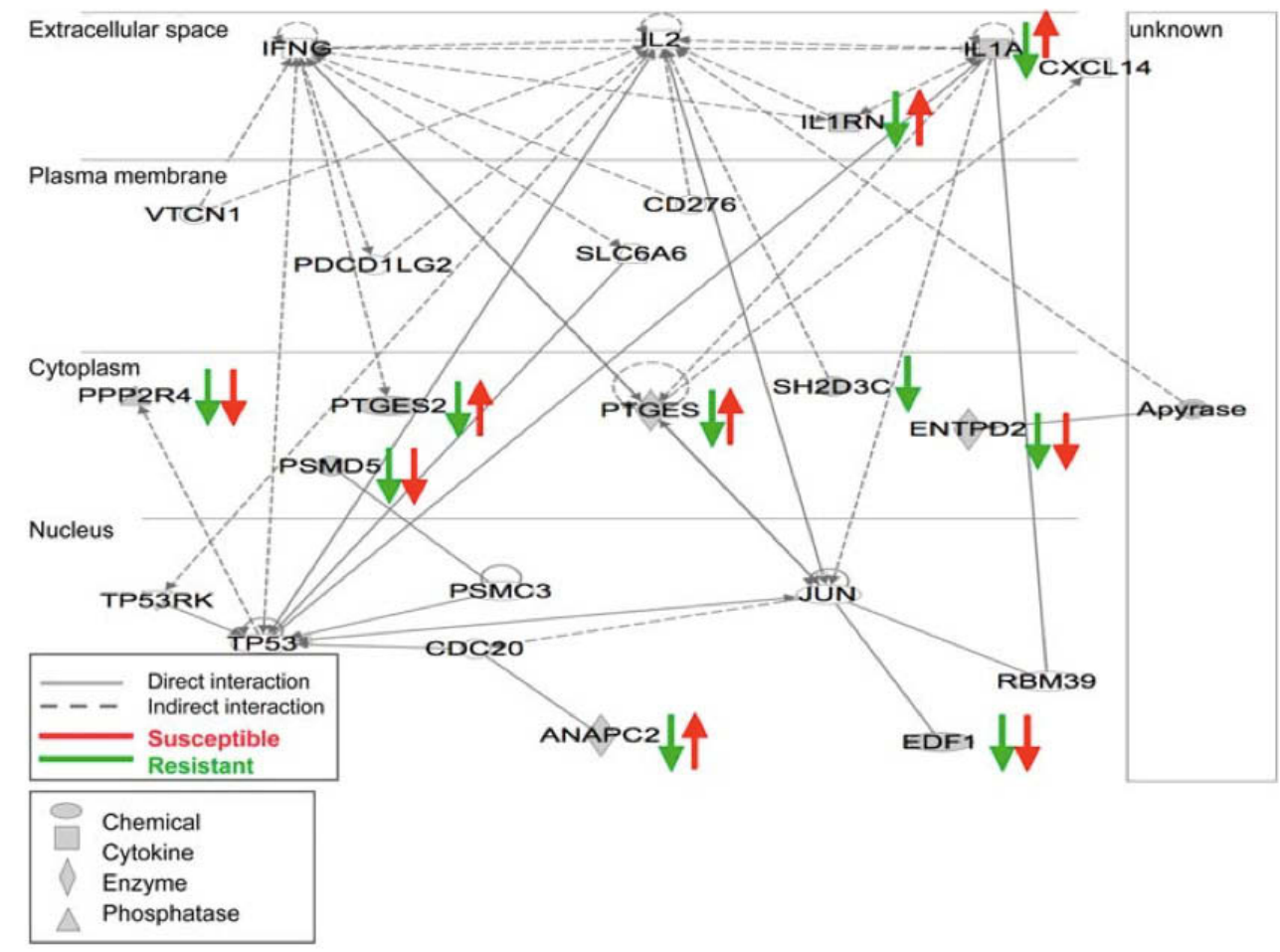

Figure 3-5. Functional network of genes modulating GAS QTL.

Graphical representation of the molecular relationships between differentially expressed genes, showing the central role of IL1, IL1rn, PTGES, and PTGES2 in modulating response to GAS sepsis and their indirect interactions with IFN $\gamma$ and IL2 networks in modulating bacterial sepsis. Genes are represented as nodes, and the biological relationship between two nodes is represented as line, solid lines represent direct interactions, dashed lines represent indirect interactions. Oval shapes represent chemical or drug, squares represent cytokines, diamond shapes represent enzymes, concentric circles represent group of family, and triangles represent phosphatases. Blue lines and arrows represent expression levels of resistant strains, while red lines and arrows represent susceptible strains expression levels. Apyrase, ATP diphosphohydorlase; ANAPC2, anaphase promoting complex subunit 2; CDC20 cell division cycle homolog 20; CXCL14, chemokine (c-X-c motif) ligand 14; ENTPD2, ectonucleoside triphosphate diphosphohydrolase 2; EDF1, endothelial differentiationrelated factor 1; JUN jun oncogene; IL1A, interleukin 1 alpha; IL1RN, interleukin 1receptor antagonist; IFNG interferon gamma; IL2 interleukin2; PDCD1LG2, programmed cell death ligand 2; PTGES, prostaglandin E synthase; PTGES2, prostaglandin E synthase 2; PSMC3 proteasome (prosome, macropain) 26S subunit ATPase 3; PSMD5, proteasome (prosome, macropain) 26S subunit, non-ATPase 5; PPP2R4, protein phosphatase 2A regulatory subunit B; RBM39, RNA binding protein 39; SH2D3C, SH2 domain containing 3C; SLC6A6, solute carrier family 6; TP53 tumor protein p53; TP53RK, TP53 regulating kinase; VTCN1, V-set domain containing $\mathrm{T}$ cell activation inhibitor 1 . 
ARI strains used in this study afford one of the best unbiased forward genetics approaches to determine how different combinations of polymorphic genes interact to shape disease phenotypes. Using ARI-BXD panel as a genetically diverse reference population, we were able to map QTLs modulating specific phenotypes associated with severe GAS sepsis.

GAS causes a wide range of diseases depending on multiple factors including site and route of infection, interplay of the pathogen virulence factors with host immune defenses that are affected by the host immune status and difference in the genetic makeup of the host (Kotb et al., 2002). Thus, these bacteria represent a good model to explore the impact of host complex traits on susceptibility to infections. Previous studies have shown that host-pathogen interactions modulate the severity of GAS sepsis (Chatellier et al., 2000; Medina et al., 2001; Kotb et al., 2002; Goldmann et al., 2004b), and we found that patients with GAS sepsis expressing HLA class II DRB1*15/DQB1*06 (DR15/DQ6) haplotype are protected from severe GAS sepsis, whereas those with HLA class II DRB1*14/DQB1*05 (DR14/DQ5) haplotype are at high risk for developing severe and often fatal forms of the disease (Kotb et al., 2002). The strong association between disease severity and HLA class II allelic polymorphism is primarily related to the differential ability of HLA class II alleles to present SAgs to T cells, where presentation of Strep SAgs by the protective HLA class II alleles results in a significantly attenuated response as compared to their presentation by the neutral or high-risk alleles (Kotb et al., 2002; Norrby-Teglund et al., 2002; Nooh et al., 2007). While this association made perfect biological sense based on the known central role of SAgs in the human disease, mice are not susceptible to SAgs due to an inherent lower affinity of mouse MHC class II molecules to GAS SAgs. The role of GAS SAgs can be well investigated in HLA class II transgenic mice as others and we have reported (Leder et al., 1998; Andersen et al., 1999; Welcher et al., 2002; Nooh et al., 2007). However, due to the overwhelming response to SAgs in these mice, it is difficult to use them to dissect host response to other GAS virulence factors in the disease process. For this reason, our present ARI mouse model of sepsis is ideal for identifying host genetic variations, besides the MHC class II antigens, that may also contribute to differences in GAS sepsis severity.

We invested time and effort to standardize the GAS infection model using a large number of BXD mice $(\mathrm{n}=717)$ and this allowed us to optimize infection dose and identify significant covariates (e.g. age and sex) needed to be accounted for in our final analysis (Aziz et al., 2007). Although GAS strains can vary considerably with regards to virulence, we showed that the same virulent strain could cause diseases with starkly different severity in humans (Chatellier et al., 2000; Kotb et al., 2002). The strain used in this study is a hypervirulent derivative of the M1T1 clonal strain that emerged in the 1980s at the same time that the severe forms of the invasive GAS infections resurged (Kazmi et al., 2001; Aziz et al., 2004a; Buchanan et al., 2006; Walker et al., 2007). Initial studies (Aziz et al., 2007) identified an optimal infection dose of $2 \pm 1 \times 10^{7}$ $\mathrm{CFU} /$ mouse, and indicated the need to use mice with an age range of 40-120 days for linear correlation with survival. In addition, these pilot studies determined that sex has insignificant effect in this GAS sepsis model (Aziz et al., 2007) and revealed a stark variation among various $\mathrm{BXD}$ strains used with respect to their susceptibility to severe 
sepsis. However, precise mapping of QTLs required that we study more BXD strains and include more mice per BXD strain to obtain statistical power.

With a total of $30 \mathrm{BXD}$ strains and an average of 15 mice per strain, we mapped QTLs on Chr 2 that modulate severity to GAS sepsis, measured by comparing mice survival post-infection, bacterial load in blood, and bacterial dissemination to spleen across the BXD strains. Inasmuch as the BXD strains are heavily genotyped with more than 3600 genomic markers, identifying QTLs is relatively straightforward. The mapped QTLs on Chr 2 harbor a relatively large number of candidate genes associated with various functional networks and signaling pathways including nuclear factor- kappa $\mathrm{B}$ (NF-KB) and p38 mitogen activated protein kinases (MAPK) pathways, proliferation of immune cells, and eicosanoid signaling. Such output is typical of unbiased genome-wide analysis studies, and required further analyses to determine which of these genes are the key modulators of the studied trait.

To narrow down the gene list to a handful of genes that can be experimentally validated, we used multiple methods including linkage, gene ontology, and differential gene expression analyses. Our quantitative PCR analysis of 37 representative candidate genes showed that 28 genes were differentially expressed in selected susceptible and resistant strains post-infection. These differentially expressed genes fell into three main categories, genes associated with innate and adaptive immune response, and genes associated with apoptosis. Differentially expressed genes associated with innate immune response were both pro- and anti-inflammatory as well as adaptive immune response genes associated with $\mathrm{T}$ and $\mathrm{B}$ cell proliferation and differentiation, cell signaling and antigen processing and presentation.

Differentially expressed genes associated with innate immune response were either related to pro- or anti-inflammatory responses. Both pro- and anti-inflammatory associated genes were up-regulated in the selected susceptible strains post-infection, and this is in agreement with a recent study by Goldmann, et al. (2007) who showed a mixed pro- and anti-inflammatory response belonging to M1 and M2 macrophage phenotypes in response to GAS infection. This increase in both pro- and anti-inflammatory response could be attributed to homeostatic mechanisms where, for example, the increase in Ill levels in the susceptible strains was accompanied by an increase in its endogenous antagonist Ill rn. This was not the case in the resistant strains that showed decreased levels of expression of pro- and anti-inflammatory related genes both pre- and postinfection. These findings suggest that susceptibly to GAS sepsis is associated with an overzealous innate immune response as observed in susceptible BXD strains only. These results mirror previous findings in humans, where association of exaggerated inflammatory responses, including IL1, with susceptibility to GAS sepsis was detected (Norrby-Teglund et al., 2000; Norrby-Teglund and Kotb, 2000; Kotb et al., 2002).

However, unlike what we found in this mouse model, human responses are dominated by high levels of IFN $\gamma$ and TNFß, presumably because the human disease is driven primarily by SAgs (Norrby-Teglund et al., 1997; Norrby-Teglund et al., 2000).

In general, differentially expressed genes associated with early adaptive immune 
responses showed a pattern of decrease in expression levels in both susceptible and resistant strains with the exception of anaphase promoting complex subunit 2 (Anapc2) that was slightly increased in susceptible strains post-infection (Figure 3-5). By contrast, several genes associated with pro- and anti-apoptotic response were differentially expressed in the selected susceptible and resistant strains post-infection (Figure 3-5). Apoptosis in streptococcal pathogenesis is affected by interacting factors including, context of infection (Marriott and Dockrell, 2006), cells undergoing apoptosis (Kobayashi et al., 2003; Nakagawa et al., 2004; Miyoshi-Akiyama et al., 2005), for example, apoptosis aids in the clearing of infection if macrophages are undergoing apoptosis, while it would be harmful to the host if lymphocytes are undergoing apoptosis. Other factors include, whether the bacteria is internalized or extracellular (Cywes Bentley et al., 2005) and accordingly the type of apoptosis pathways activated (Klenk et al., 2005). In our murine GAS sepsis model, we have measured expression levels in whole spleens, which involved the response of various cells including macrophages, $\mathrm{T}$ and $\mathrm{B}$ lymphocytes, dendritic and endothelial cells etc. Consequently, we expected to observe a mixed response; however, in our ongoing studies, we are examining possible alterations in splenic population profiles post-infection in the various BXD strains to determine which cell types are responsible for the major differences in cytokine levels seen postinfection and to dissect the role of different cell populations in this GAS sepsis model.

Another interesting observation was that the relative expression level of genes measured in the selected resistant strains showed a pattern of decrease post-infection, with the exception of five genes, heat shock 70KDa protein 5 (Hspa5), Traf1, Traf2, Notch gene homolog 1 (Notch1), and signal-regulatory protein alpha (Sirpa). It is worthy to note the link between these genes, as they are involved directly and indirectly with activation of early adaptive response. Hspa5 is an Hsp70, which is associated with cytoprotection, anti-apoptosis, and anti-inflammation (Kiang and Tsokos, 1998; Yenari et al., 2005; Kim et al., 2006), and has been associated with immune response to sepsis (Kustanova et al., 2006; Singleton and Wischmeyer, 2006). Notch1 has been associated with the signaling involved in regulation of lymphocytes development and activation to effector cells (Minato and Yasutomo, 2005), natural killer cells development (van den Brandt et al., 2004; Garcia-Peydro et al., 2006; Rolink et al., 2006) and recently Notch1 was associated with macrophage activation (Monsalve et al., 2006). We took into consideration in our experimental design that stress might alter the expression of stress related genes especially heat shock proteins and chemokines, therefore, we subjected control mice to the same stress as infected mice by injecting control mice with saline, so that any change in expression levels would be accounted to GAS sepsis.

Among the differentially expressed genes, four genes, Ill, Il1rn, Ptges, and Ptges2, showed marked up regulation in susceptible strains, while they showed no change or slight decrease in levels in resistant strains post-infection (Figure 3-5). Increase in expression of Ptges and Ptges 2 genes is induced by the increased levels of Ill (Bream et $a l ., 2000$ ), which is an indirect effect of the activation of IFN $\gamma$ (Figure 3-5). Ptges and Ptges 2 are prostaglandin synthases for the lipid inflammatory mediators PGE2, which along with platelet activating factor and leukotrienes; mediate vasodilatation in the early response to inflammation (Harris et al., 2002; Mosca et al., 2007). Vasodilatation in turn 
leads to hypotension, a hallmark of STSS. Although the role of prostaglandins in inflammation and immune response has long been studied, their role in the immune response to infectious diseases has been lately pursued (Ruiz et al., 1998; Maloney et al., 2000; Smith et al., 2002; Chen et al., 2004; Jobin et al., 2006; Woolard et al., 2007). Increased production of prostaglandins has been associated with various Gram positive bacterial infections including Streptococcus suis (Jobin et al., 2006), group B streptococcal (Maloney et al., 2000), and GAS skin infections (Ruiz et al., 1998).

In conclusion, our holistic approach of studying the genetic basis of differential susceptibility to GAS sepsis revealed loci on Chr 2 that harbor major immune modulators. In the present study, we examined the interactions of pathogen multiple virulence factors with the host immune system in an in vivo model of sepsis using a genetically diverse reference population. This shed light on a network of host genes modulating variation in GAS severity, which includes cytokines, pro- and antiinflammatory mediators, and genes associated with apoptosis and early adaptive immune response. Our ongoing detailed studies to identify interactive molecular pathways contributing to the complex trait of GAS sepsis will undoubtedly help us dissect the various mechanisms by which the host interacts with the bacteria in vivo, resulting in resistance or increased susceptibility to severe GAS sepsis.

\section{Materials and Methods}

\section{Mice}

We generated the BXD advanced recombinant inbred (ARI) mice at UTHSC by crossing B6 and D2 mice to generate F1 hybrids, which were crossed to generate F2 progeny, each with random patterns of recombination. Random crossing of F2 mice generated F3 and so on, till F11 generation, after which we designated pairs of F11 hybrids as parents of each BXD line, and inbred them by sib mating for $>20$ generations to achieve homozygosity for each genetically distinct BXD line. This breeding scheme was done to increase recombination events resulting in roughly double the number of recombinations per strain compared to conventional RI strains (Williams et al., 2001; Peirce et al., 2004).

The genomes of the B6 and $\mathrm{D} 2$ parental strains have been sequenced and a database of their SNPs is available at the Gene Network (GN) web site (www.genenetwork.org/webqt1/snpBrowser.py). Simple sequence length polymorphism (SSLP) markers were typed for all BXD RI strains as previously described (Williams et al., 2001). The BXD progeny is genotyped at 13377 SNPs and microsatellite markers (Shifman et al., 2006), a selected subset of 3795 SNPs and microsatellite markers used by GN BXD genotype dataset for mapping traits, can be downloaded at www.genenetwork.org/genotypes/BXD.geno.

We recently standardized the model of BXD ARI mice for use in GAS infection studies (Aziz et al., 2007). In the current study, a total of 696 mice were used, from 
which 183 flagged mice were excluded based on predetermined criteria as previously detailed in (Aziz et al., 2007). All procedures involving mouse tissues were approved by the institutional animal care and use committee at the UTHSC.

\section{Experimental Design}

Population-based experiments

Groups of 5-26 mice from a total of $30 \mathrm{BXD}$ lines and their parental strains (B6 and D2) were injected via tail vein with $2 \pm 1.5 \times 10^{7} \mathrm{CFU} /$ mouse of a hypervirulent form of the clinical isolate GAS 5448 (M1T1) (Chatellier et al., 2000; Aziz et al., 2004a; Aziz et al., 2004b) as detailed previously (Aziz et al., 2007). Mortality and weight loss were recorded every $8 \mathrm{~h}$ for the ensuing 6 days. To normalize across experiments, we inspected survival days distribution clusters for each experiment and determined multimodal distribution and boundaries of each cluster for a total of three clusters: susceptible, intermediate, and resistant. Survival days within each cluster were then converted into a survival index ranging from $0.25-1,1.25-2$, and $2.25-3$ for susceptible, intermediate, and resistant clusters respectively. The survival index was assigned to each mouse irrespective of its strain. Indices for each strain, across experiments were then corrected for significant covariates (age, sex, body weight, and inocula) using multiple regression analyses.

Phenotype assessment experiments

We designed experiments for the assessment of survival, bacteremia, and bacterial dissemination to spleen. We assessed relative mice survival and bacteremia in the same experiment. We measured bacteremia as $\mathrm{CFU} / \mathrm{ml}$ by culturing serial dilutions of blood drawn from mice tails $24 \mathrm{~h}$ post-injection. We determined bacterial load in spleen by homogenizing spleens of expired mice using tissue homogenizer, TH (Omni International, Marietta, GA). Each tissue homogenate was serially diluted 10 folds to $10^{-6}$ fold, and each dilution was cultured for determination of bacterial load in spleens $(\mathrm{CFU} / \mathrm{ml})$ that was then normalized to spleen weight and expressed as CFU/spleen.

\section{Quantitative Trait Loci Mapping}

We performed QTL mapping using web-based complex trait analysis available on the GN website and the mapping module which analyzes phenotypes in context of mouse genotypic differences. Interval mapping evaluates potential QTL at regular intervals and estimates the significance at each location using 1000 permutation tests (Churchill and Doerge, 1994). We performed three sets of analyses using strain means for the following three variables: (1) corrected relative survival index, described earlier, (2) log bacterial load in blood $24 \mathrm{~h}$ post injection, and (3) log bacterial load in spleen at expiration. 


\section{Quantitative PCR Analysis for Target Genes Expression}

We investigated the differential expression of target genes in spleen of infected vs. control PBS-injected mice at selected time points; we selected strains based on their susceptibility, BXD61 and 90 representing susceptible strains, and BXD73 and 87, representing resistant strains. We performed 2-3 biological replicates of each set of paired susceptible and resistant $(\mathrm{n}=6-8$ per strain). Mice were sacrificed $40 \mathrm{~h}$ post i.v. injection with $2 \pm 1.5 \times 10^{7} \mathrm{CFU} /$ mouse of clinical isolate GAS 5448 (M1T1) and RNA from individual mice was extracted from spleens. Bacteremia was determined as described above. We isolated RNA using RNA-STAT 60 method and when necessary, we purified RNA samples using RNeasy mini kit clean up columns (Qiagen, Valencia, CA). We pooled RNA samples per strain with A260/280 ratios $\geq 1.8$ for cDNA synthesis with SuperScript III reverse transcriptase kit (Invitrogen, Carlsbad CA) using oligo dT primers. We designed real time PCR assays, hydrolysis probes, and gene specific primers that span long introns to distinguish cDNA from genomic DNA using primer design online software universal probe library (UPL) at www.roche-appliedscience.com/sis/rtpcr/upl/index.jsp. We performed quantitative TaqMan PCR on light cycler LC480 (Roche Applied Science, Indianapolis, IN). We used the mouse housekeeping gene, hypoxanthine guanine phosphoribosyl transferase (Hprt l) as an endogenous control to which we normalized gene expression data. Primer sequences are listed in Appendix A Table A-1. Samples were analyzed in triplicates for each of 2-4 biological replicates. We used delta delta $\mathrm{Ct}$ (threshold cycle) method for calculating relative expression levels expressed as fold differences between pre- and post-infection values for each gene analyzed. Student t-test was used to assess statistical significance.

\section{Identification of Differentially Expressed Genes in the Mapped Interval and Bioinformatics Functional Pathways Analyses}

We generated functional analyses of genes within the QTLs using Ingenuity pathways analysis (IPA) (www.ingenuity.com). Each data set containing gene identifiers was uploaded into the online application, and each gene was overlaid onto a molecular network developed from information contained in the ingenuity pathways database. Networks of genes were then generated based on their connectivity, and we chose the top 50 significant networks. The significance of the association between the data set and the pathways was measured in two ways: (1) the ratio of the number of genes from the data set that map to the pathway divided by the total number of genes that map to the pathway; and (2) by Fischer's exact test with $\mathrm{P}<0.001$.

\section{Statistical Analysis}

We used the DataDesk 6.2 (www.datadesk.com) to calculate mean survival indices, corrected survival indices, and correlation coefficients. Covariates association was evaluated for significant associations by Spearman correlation as described previously (Aziz et al., 2007). Fischer exact test was used to analyze association of the analyzed differentially expressed genes with specific pathways. 


\section{Web Site URL}

Our data sets stored in WebQTL can be found at Gene Network (www.genenetwork.org) under BXD published phenotypes record ID 10836. 


\section{CHAPTER 4. IDENTIFICATION OF SOLUBLE AND GENETIC BIOMARKERS ASSOCIATED WITH DIFFERENT OUTCOMES OF GAS SEPSIS REVEALS UNDERLYING MECHANISMS OF DIFFERENTIAL HOST SUSCEPTIBILITY}

\section{Summary}

Individuals infected with the same group A streptococcal (GAS) strain can develop starkly different disease progression and outcomes. My mentor's laboratory have shown that the same GAS strain can cause a wide range of diseases, from mild bacteremia and erysipelas to streptococcal toxic shock syndrome (STSS) and necrotizing fasciitis (NF) (Chatellier et al., 2000). Difference in disease severity was associated with differences in the profile and magnitude of cytokine responses, where overzealous inflammatory cytokine responses, triggered mainly by streptococcal superantigens (SAgs), were associated with severe disease, including STSS and NF (Norrby-Teglund et al., 2000). Our group has found that HLA class II allelic variations contributed to the differences in cytokine responses in these patients, where individuals with DR14/DQ5 HLA class II haplotypes, produced high levels of inflammatory cytokines and were at risk of developing severe systemic disease and STSS. Whereas, we found that patients who had DR15/DQ6 haplotype mounted moderate cytokine responses and experienced mild sepsis and/or cellulitis (Kotb et al., 2002; Norrby-Teglund et al., 2002).

The HLA-II association was related to interactions of GAS SAgs with the host where GAS SAgs forced interactions of HLA-II on antigen presenting cells and T cell receptors on $\mathrm{T}$ helper cells stimulating both types of cells to mount cytokine responses which differed based on HLA-II alleles as described above. However, GAS is rich in many other virulence factors that are likely to engage other host defense mechanisms. Indeed, we have recently shown that additional host factors, other than HLA- II, are modulating host differential responses to GAS infections. Employing genome-wide association studies, we mapped differential susceptibility to severe GAS sepsis to QTLs on mouse Chr 2 and X (Abdeltawab et al., 2008). We found that within these QTLs most significant association with differential susceptibility to GAS sepsis was that of interleukin 1 and prostaglandin E pathways. We validated our results based on genomewide association studies (GWAS) using multiple bioinformatics tools and quantitative gene expression analyses (as detailed in chapter 3 ). However, we aimed to perform genome-wide expression analyses to study patterns associated with differential responses to GAS sepsis at early stages of infection. In the current chapter, we aimed to further dissect the mechanisms of the discovered pathways (discussed in chapter 3) and how they are associated with differential susceptibility. In the studies covered in this chapter, in addition to analyzing genome wide (GW) gene expression patterns, we also analyzed soluble biomarkers that are associated with pathways identified by both GW mapping and GW expression analyses. 


\section{Results and Discussion}

\section{Comparison of Genome-wide Differential Expression in Blood between Susceptible and Resistant Strains at Selected Times Post Infection}

In studies outlined in this dissertation, our overall aim was to investigate mechanisms underlying differential response to GAS sepsis. We employed genome-wide association studies (GWAS) adopting systems genetics approach as discussed in chapters 2 and 3 . Using these genome-wide association studies, we identified interleukin 1 and prostaglandin pathways as candidate pathways associated with modulation of differential susceptibility to severe GAS sepsis (chapter 3 and Abdeltawab et al. 2008). In this chapter, we sought to characterize and further dissect genetic and soluble biomarkers associated with differential susceptibility to GAS sepsis. To do so, we first aimed to characterize differential gene expression patterns associated with differential susceptibility to GAS sepsis. We compared global gene expression differences (genetic biomarkers) and differences in a selected set of cytokines associated with sepsis (soluble biomarkers) between uninfected vs. infected mice of selected highly resistant and highly susceptible strains at 8,12 , and 24 hours post infection.

In our global gene expression analyses, we aimed to profile patterns associated with differential susceptibility to severe GAS sepsis. To achieve this aim, we compared infected mice at different stages of infection ( 8 and 12 hours post GAS infection) to uninfected group of mice (0hrs) (experimental design illustrated in Figure 4-1). We used groups of ARI-BXD strains that displayed marked differences in response to severe GAS sepsis as characterized in studies outlined in chapters 2 and 3 of this dissertation and Aziz et al. 2007 and Abdeltawab et al 2008. We designed our experiments so that there were multiple levels of replication. First at the level of ARI-BXD strains used, we selected three highly susceptible ARI-BXD strains (BXD97, 100, and 77) ${ }^{*}$ to represent the most susceptible side of strains (see gradient of differential susceptibility in Figure 3-1). Similarly for the resistant group, we chose to use three most resistant ARI-BXD strains (BXD48, 73, 87) ${ }^{\dagger}$. In doing so, there were replicas on the level of both susceptible and resistant groups, instead of one strain representing the susceptible group; we collected data from three extremely susceptible strains and similarly for resistant strains as illustrated in Figure 4-1. Within each of these selected representative six strains, we collected and pooled blood from three mice per strain. Each pool of blood represented one strain at a selected time point; this represented biological replica ( 3 mice per strain). To guard against effects of stress due to multiple bleedings of the same mouse, we used three different sets of mice ( 3 different mice per set) to analyze each of the studied time points whether pre infection or at different stages of infection (Figure 4-1).

\footnotetext{
${ }^{*}$ According to our studies outlined in chapter 3 as illustrated in Figure 3-1, BXD94 and BXD90 are among the extremely susceptible strains; however we did not use BXD94 and BXD90 as BXD94 is now extinct, while BXD90 has been recently difficult to breed in our hands.

${ }^{\dagger}$ Similarly for resistant strains, we used strains that exhibit extreme resistance as illustrated in Figure 3-1 (BXD48,, BXD73 and BXD87), however, we did not use BXD92 which is also extremely resistant as it has become extinct.
} 
Figure 4-1. Experimental design to test gene expression differences associated with differential susceptibility to severe GAS sepsis at selected stages of infection.

Illustration of experimental design used for the assessment of patterns of differential gene expression in blood associated with differential susceptibility to GAS sepsis at early stages of infection ( 8 and 12 hours post infection). We selected mice belonging to highly resistant strains (BXD48, 73 and 87) and highly susceptible strains (BXD100. 97 and 77). Strains were chosen based on our previous differential susceptibility results detailed in chapter 3 of this dissertation. At each time point, $0 \mathrm{~h}$ representing uninfected mice and 8 and 12 hours post infection, we sacrificed three mice per strain and collected blood that was later pooled per strain, representing certain strain at a certain time point (e.g. BXD48 in the illustration, where RNA1 represents BXD48 $0 \mathrm{~h}$ and RNA2 represents BXD48 8h, each RNA sample was prepared from pooled blood of 3 mice). The same design was repeated for each and every strain. We extracted RNA from these pooled blood samples and hybridized purified high quality RNA to Illumina WG-6 V2.0 mouse arrays (see methods for details of arrays processing). We compared differentially expressed genes in the resistant group (as a whole) at 8 and 12 hours post infection to uninfected mice, and obtained a list of differentially expressed genes, denoted as $\mathrm{A}$ and $\mathrm{B}$ for resistant strains at 8 and $12 \mathrm{~h}$ respectively. Similarly, gene lists C and D for susceptible strains at 8 and 12 hours respectively as shown. We then parsed these differentially expressed gene lists associated with resistance and those with susceptibility into pathways. These pathways are shown in Figures 4-2 and 4-3, and methods used to generate these pathways are detailed in methods section. 


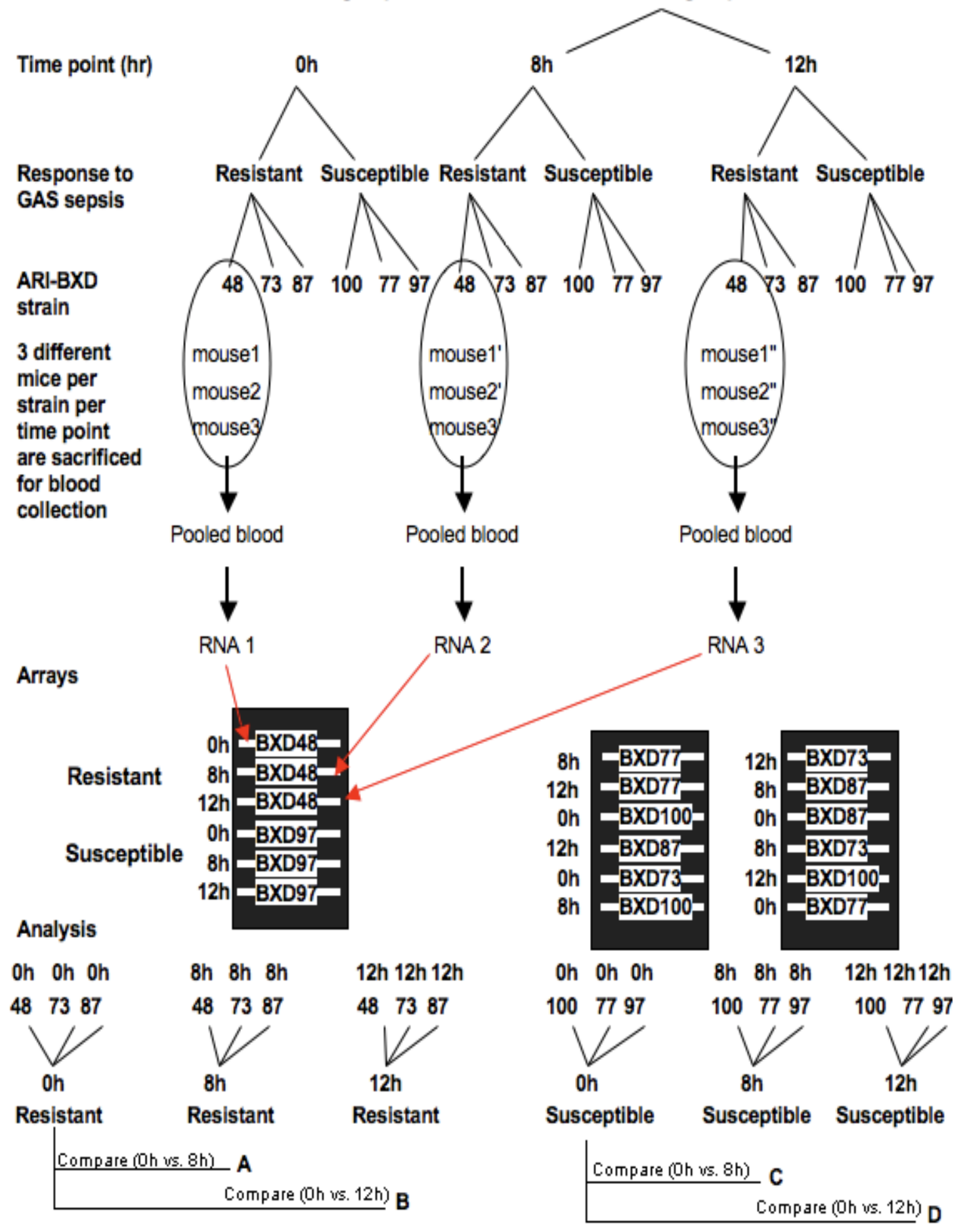


We adopted the design (illustrated in Figure 4-1) to allow us to investigate patterns of differentially expressed genes associated with differential susceptibility to severe GAS sepsis. In addition we wanted to investigate stage-specific differential gene expression patterns associated with differential susceptibility.

We chose blood for our current analyses of early stage of infection as our genome-wide association studies revealed association of differential susceptibility to the innate immune cells, neutrophils and monocytes. In addition, blood is the main organ that mounts the first line of defense to this blood borne pathogen. Nonetheless, we also collected data for differentially expressed genes in the spleens of above-mentioned selected highly susceptible and highly resistant strains at multiple times post infection representing different stages of infection. We chose to also investigate genome-wide differential gene expression in the spleen due to the spleen role as one of the main secondary lymphoid tissues involved in clearance of extracellular blood borne bacteria. In future studies to be performed in the laboratory, we plan to also investigate differential gene expression in lungs of these differentially susceptible and resistant strains, as the lung is one of the main organs involved in the severe outcome of GAS sepsis associated with high mortality in clinical settings manifested by acute respiratory distress syndrome.

In our genome-wide differential gene expression analyses of blood, we aimed to analyze stage-specific patterns of resistant strains and susceptible strains compared to their uninfected control (Figure 4-1). For example gene expression levels at 8 hours post infection from three resistant strains compared to $0 \mathrm{hr}$ (uninfected mice) of the same strains. Similarly for susceptible strains at 8 hours vs. 0 hours and also we performed similar analyses for $12 \mathrm{hrs}$ compared to $0 \mathrm{hrs}$ for both resistant and susceptible groups (Figure 4-1).However, our current design allows us to analyze the data we generated in multiple ways asking different questions. For instance, we can ask the question of what are the patterns of different mechanisms of resistance by comparing the sets of differentially expressed genes of each of the tested three resistant strains, and similarly for susceptible strains. Another question we would like to address is: what are the differences in gene expression in blood cells between susceptible and resistant group preinfection (0h). This will help us address the question of possible predisposition to severe sepsis prior to GAS infection.

We compared global gene expression in blood at 8 hours vs. 0 hours and found that 232 genes were differentially expressed in resistant strains, while 322 genes were differentially expressed in blood cells of susceptible strains (Figure 4-1, 4-2 and Table 4-1). Comparing these two differentially expressed gene lists, we found that there were 91 genes that were shared (common) between differentially expressed gene sets of susceptible and resistant strains (Figure 4-2). These shared genes fell into multiple pathways, including interferon signaling and interferon regulatory factor activation pathway. Both pathways were upregulated in both susceptible and resistant strains (Figure 4-2 and Table 4-1). However, there were quantitative differences between susceptible and resistant strains in this upregulation (Table 4-1). For example, interferon induced protein with tetratricopeptide repeats 3 (Ifit3) was upregulated at $8 \mathrm{~h}$ vs. $0 \mathrm{~h}$ in 
Figure 4-2. Pathways associated with differentially expressed genes at 8 hours post infection in peripheral blood of resistant and susceptible strains compared to uninfected (0hr) mice of respective strains.

Panel A. Venn diagram of differentially expressed genes in blood of resistant and susceptible strains, showing common (shared) and unique genes. Resistant strains had 232 genes differentially expressed within these, 141 genes were unique to resistant strains while 91 genes were shared with susceptible strains. A total of 322 genes were differentially expressed in susceptible strains within these genes, 231 genes were unique to susceptible strains. These lists of differentially expressed genes were parsed into pathways using ingenuity pathway analysis tool (IPA) as detailed in methods section. Shown are the most significant pathways. We parsed common (shared) differentially expressed genes in both resistant and susceptible strains into pathways shown in panel B. The significant pathways associated with genes that are differentially expressed only in resistant strains are shown in panel $\mathrm{C}$, while those associated only with susceptible strains are shown in panel D. Whether the genes within a pathway are up or down regulated is denoted in words and as arrows beside each pathway. The $\mathrm{x}$-axis displays significance in (-log (p-value)) where the higher the number (the longer the blue bar) the more likely the association of the differentially expressed genes with the noted pathways is not by chance, this significance is also expressed as p-values on each bar. For each pathway we calculated a ratio (orange squares, and decimal number associated with it), the ratio is the number of differentially expressed genes divided by total number of molecules in the specified pathway. 
A Venn diagram of differentially expressed genes at $8 \mathrm{~h}$ post infection in blood of resistant and susceptible strains, showing common (shared) and unique genes

Differentially expressed genes in resistant $(\mathrm{R}) /$ susceptible(S) strains at 8 hrs vs. uninfected $(0 \mathrm{~h})$ mice

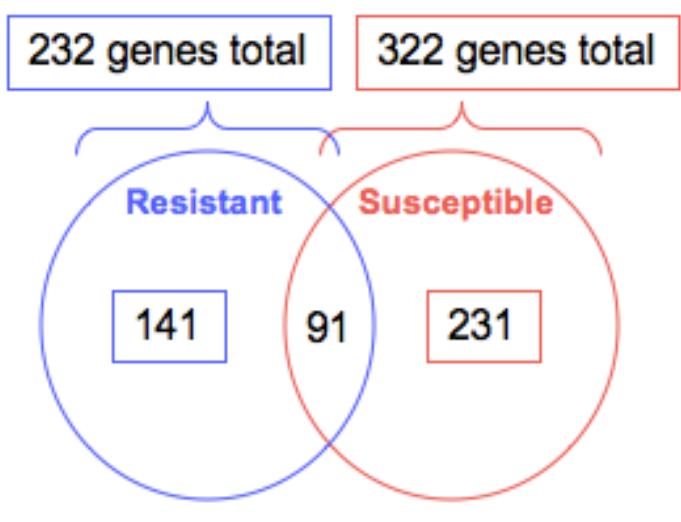

B Pathways of differentially expressed genes shared (common) between resistant and susceptible strains at $8 \mathrm{~h}$ post infection

\section{Common pathways $8 \mathrm{hr}$ post infection}

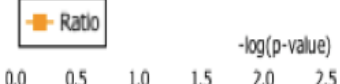

Activation of interferon regulatory factor (IRF)

family by cytosolic pattern recognition receptors upregulated

Interferon signaling - upregulated

Protein ubiquitination pathway - upregulated

Death receptor signaling - upregulated

Induction of apoptosis - upregulated

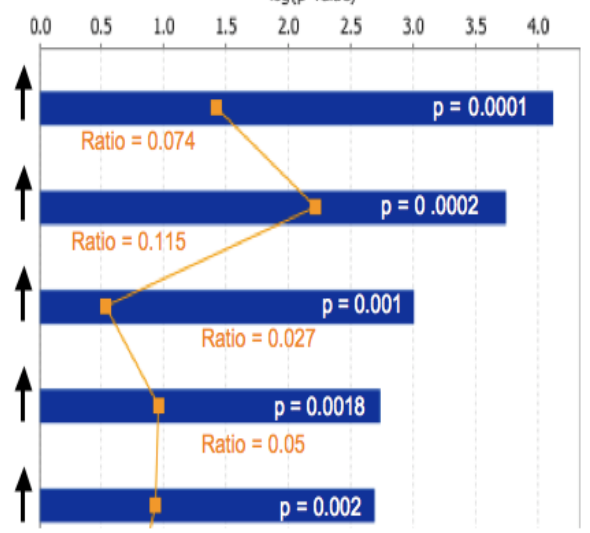

Ratio $=0.048$ 
C Pathways of differentially expressed genes associated with resistant strains at $8 \mathrm{~h}$ post infection

\section{Resistant strains $8 \mathrm{hr}$ post infection}

\section{IL-10 signaling - upregulated}

Role of protein kinase receptor $(\mathrm{PKR})$ in interferon induction and antiviral response upregulated

Hepatic cholestasis - upregulated

Role of Macrophages, fibroblasts and endothelial cells in rheumatoid arthritis - upregulated

PPAR (peroxisome proliferator-activated receptor) family signaling - upregulated

TNFR1 (Tumor Necrosis Factor Receptor) family signaling - upregulated
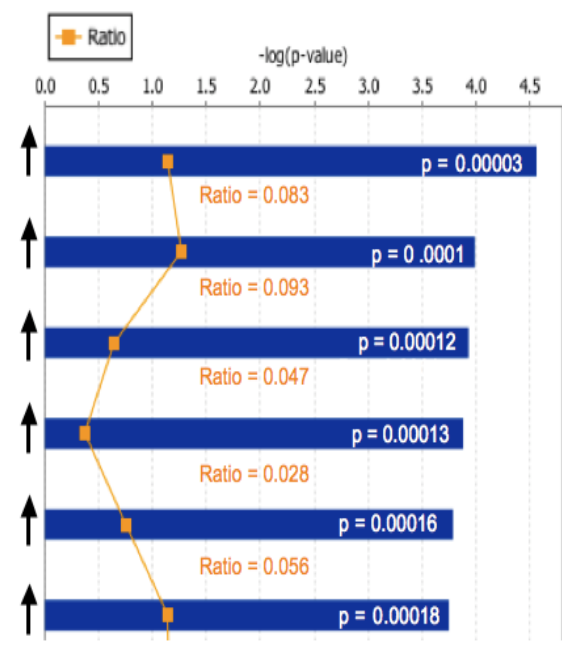

Ratio $=0.083$
D Pathways of differentially expressed genes associated only with susceptible strains at $8 \mathrm{~h}$ post infection

Susceptible strains $8 \mathrm{hr}$ post infection

Role of protein kinase receptor ( $\mathrm{PKR}$ ) in

interferon induction and antiviral response downregulated

CD40 signaling - downregulated

Mitochondrial dysfunction — upregulated

Glycosaminoglycan degradation - upregulated

Protein ubiquitination pathway - upregulated

Role of macrophages, fibroblasts and endothelial cells in rheumatoid arthritis - upregulated

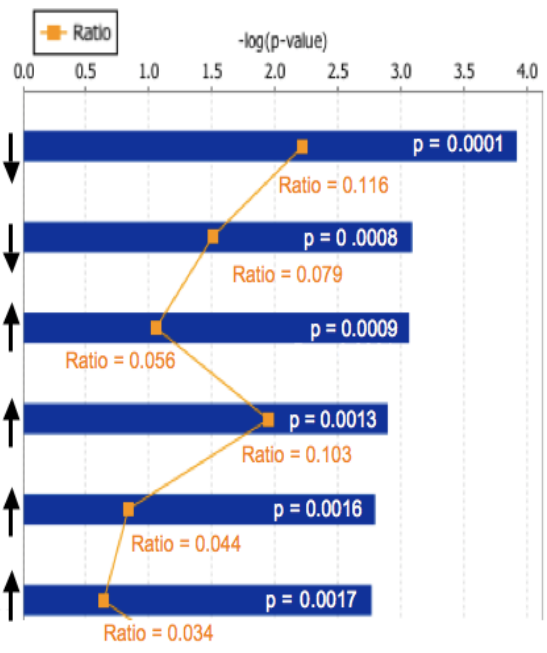

Figure 4-2. (Continued). 
Table 4-1. Differentially expressed genes in peripheral blood of resistant and susceptible strains at 8 hrs post GAS infection.

\begin{tabular}{|c|c|c|c|}
\hline \multirow[t]{2}{*}{ Pathways and Genes/Transcripts } & \multirow[t]{2}{*}{ Gene/Transcript Description } & \multicolumn{2}{|c|}{ Fold Change } \\
\hline & & Susceptible & Resistant \\
\hline \multicolumn{4}{|c|}{$\begin{array}{l}\text { Top pathways associated with differentially } \\
\text { expressed genes shared between susceptible } \\
\text { and resistant strains } \\
\text { Interferon signaling and activation of IRF by } \\
\text { cytosolic pattern recognition receptors }\end{array}$} \\
\hline Ifit 3 & $\begin{array}{l}\text { Interferon-induced protein with tetratricopeptide } \\
\text { repeats } 3 \text { (Ifit3) }\end{array}$ & 19.83 & 29.42 \\
\hline Ifit2 & $\begin{array}{l}\text { Interferon-induced protein with tetratricopeptide } \\
\text { repeats } 2 \text { (Ifit2) }\end{array}$ & 5.58 & 9.57 \\
\hline Stat2 & $\begin{array}{l}\text { Signal transducer and activator of transcription } 2 \\
\text { (Stat2) }\end{array}$ & 4.04 & 3.42 \\
\hline Zbpl & Z-DNA binding protein 1 (Zbp1) & 4.13 & 3.93 \\
\hline Dhx 58 & DEXH (Asp-Glu-X-His) box polypeptide 58 (Dhx58) & 8.50 & 8.79 \\
\hline Oas $1 \gamma$ & 2'-5' oligoadenylate synthetase $1 \mathrm{G}$ (Oas 1 gamma) & 12.14 & 11.48 \\
\hline \multicolumn{4}{|c|}{$\begin{array}{l}\text { Top pathway associated with differentially } \\
\text { expressed genes in resistant strains ( } 8 \mathrm{~h} \text { vs. 0h) } \\
\text { IL10 signaling }\end{array}$} \\
\hline $\operatorname{Arg} 2$ & Arginase, type II & & 3.78 \\
\hline Ikbke & $\begin{array}{l}\text { Inhibitor of kappa light polypeptide gene enhancer in } \\
\text { B-cells, kinase epsilon }\end{array}$ & & 3.79 \\
\hline$I l 1 b$ & Interleukin 1 , beta & & 5.99 \\
\hline Illrn & Interleukin 1 receptor antagonist & & 9.49 \\
\hline $\operatorname{Tnf}$ & Tumor necrosis factor (TNF superfamily, member 2) & & 4.63 \\
\hline Jun & Jun oncogene (Jun) & & 2.23 \\
\hline
\end{tabular}


Table 4-1 (Continued).

\begin{tabular}{|c|c|c|c|}
\hline \multirow[t]{2}{*}{ Pathways and Genes/Transcripts } & \multirow[t]{2}{*}{ Gene/Transcript Description } & \multicolumn{2}{|c|}{ Fold Change } \\
\hline & & Susceptible & Resistant \\
\hline $\begin{array}{l}\text { Top pathway associated with differentially } \\
\text { expressed genes in susceptible strains ( } 8 \mathrm{~h} \text { vs. 0h) }\end{array}$ & & -2.03 & \\
\hline Role of PKR in Interferon induction & & -2.53 & \\
\hline$I k b k \gamma$ & $\begin{array}{l}\text { Inhibitor of kappa light polypeptide gene enhancer in } \\
\text { B-cells, kinase gamma }\end{array}$ & & \\
\hline Map $2 k 3$ & Mitogen-activated protein kinase kinase 3 & & \\
\hline
\end{tabular}


susceptible group by $\sim 20$ folds vs. 29 folds in resistant group (Table 4-1). Similarly other quantitative differences between susceptible and resistant group were observed.

However, we didn't observe this quantitative difference between resistant and susceptible group at 12 hours post-infection (Table 4-1 and 4-2). Further studies, which are beyond the scope of this dissertation, are needed to elucidate if and how these observed quantitative differences relate to differential susceptibility to GAS sepsis.

Among the top pathways (pathways with highest significance) associated with differentially expressed genes in resistant strains (8 vs. 0 hours) was IL10 signaling pathway (Figure 4-2 and Table 4-1). We found that most of the differentially expressed genes in IL10 signaling pathway were upregulated (Table 4-1). Of note was the upregulation of arginase II (Arg II) in resistant strains at $8 \mathrm{~h}$ post infection (Table 4-1). Arg II and nitric oxide synthase (NOS) compete for the same arginine pool with the production of L-ornithine or nitric oxide (NO) respectively. Therefore, upregulation of Arg II in resistant strains might reflect a plausible mechanism of resistance, where upregulation of Arg II might regulate the production of NO in endothelial cells by decreasing the bio availabity of arginine. This suggested that resistant strains might be upregulating Arg II to control a possible over production of NO. Over production of NO can lead to an increase in vascular permeability and leakage, leading to increase loss of fluids from vascular vessels, leading to hypotension which leads to shock, and consequently STSS.

In addition, association of upregulation of IL10 signaling pathway with resistance (Figure 4-2) suggested that resistance might be initiated early on in infection with known involvement of IL10 signaling pathway in induction of tissue repair and its immunomodulatory responses. However, in our analyses, we did not find upregulation of Il10 gene itself upregulated; although its signaling pathway was upregulated. In addition to IL10 signaling pathway, multiple pathways associated with tissue development, cell proliferation, activation of macrophages, pattern recognition activation were associated with resistance (Figure 4-2). We also found that pathways associated with bacterial killing and reactive oxygen species (ROS) production were among pathways associated with differentially expressed genes in resistant strains only. It seems contradictory that we found that resistant strains had upregulation of both Arg II (associated with regulation of NO production) and ROS production pathways. However, it might be that these resistant strains exert an active suppression of over production of such ROS but not ROS production per se. Since overproduction of NO is associated with tissue damage and shock, resistant strains guard against these deleterious effects by using up arginine pools (Arg II upregulation), meanwhile adequate production of ROS is induced to control the bacterial infection.

In susceptible strains, we found that 231 genes were differentially expressed at $8 \mathrm{~h}$ post infection compared to $0 \mathrm{~h}$ (Figure 4-1 list $\mathrm{C}$ and 4-2). The top pathways associated with these differentially expressed genes were role of protein kinase receptor (PKR) interferon induction and CD40 signaling. Most of the differentially expressed genes belonging to both pathways were downregulated. This observed downregulation of PKR induced interferon signaling in susceptible strains at $8 \mathrm{hrs}$ is in contrast to resistant strains 
that manifested upregulation of the same pathway at same time point.CD40 signaling pathway is involved in a multitude of immune responses ranging from induction of proinflammatory cytokines to activation of cell survival and was associated in the production of IL1 and IL8 from vascular endothelial cells, in addition, it is involved in the induction of prostaglandin endoperoxide synthase 2 (Ptgs 2) and subsequent production of PGE2 (Elgueta et al., 2009). The observed downregulation of genes associated with CD40 signaling and PKR induction of interferon signaling in susceptible strains might be associated with the lack of mounting appropriate response to GAS infection.

Next, we examined differential gene expression patterns at 12 hours post infection; we found that 380 genes were differentially expressed in resistant strains at 12 h compared to uninfected mice, out of which 268 genes were unique to resistant strains and 112 genes were shared with susceptible strains (Figure 4-3).Again similar to $8 \mathrm{~h}$, the shared (common) differentially expressed genes between resistant and susceptible strains (112 genes) fell into pathways that included interferon signaling. However, the quantitative differences between susceptible and resistant strains were more towards higher levels in susceptible than resistant strains, while the reverse was true at $8 \mathrm{~h}$, e.g. Ifit3 levels at 8 vs. 12 hrs (common genes Table 4-1 and 4-2). The 268 genes differentially expressed in resistant strains only, fell into interferon signaling pathway and activation of interferon signaling by pattern recognition receptors and PKR signaling. We observed this upregulation of genes associated with interferon signaling at both 8 and 12 hours in both susceptible and resistant strains however, the mechanism of induction was different and there was a down regulation of PKR induced interferon signaling in susceptible strains at $8 \mathrm{~h}$. Further detailed studies might help in elucidating the details of mechanisms of interferon in modulating differential susceptibility.

Meanwhile, pathways associated with differentially expressed genes in the blood of susceptible strains at $12 \mathrm{~h}$ post infection were mainly associated with induction of mitochondrial enzymes, induction of apoptosis and oxidative phosphorylation. Genes within these pathways were upregulated; this upregulation of mitochondrial pathways might be associated with bacterial induced mitochondrial injury and bioenergetic failure thus leading to sepsis-associatated multiple organ failure. This is further supported by our finding that in susceptible strains mitochondrial oxidative phosphorylation enzymes e.g. NADH dehydrogenase and succinate dehydrogenase were upregulated at both 8 and $12 \mathrm{~h}$ post infection (Figure 4-2, 4-3 and Table 4-2), suggesting that mitochondrial oxidative phosphorylation is upregulated to counteract the ROS produced. Furthermore, mitochondrial apoptosis related enzymes e.g. caspase 8 and cytochrome $\mathrm{C}-1$, as well as pathways associated with induction of apoptosis were upregulated in only the susceptible strains at 12h (Figure 4-3 and Table 4-2). This simultaneous upregulation of electron transport chain (ETC) enzymes and mitochondrial-induced apoptosis signaling could be explained in terms of possible positive feedback between the cascades of oxidative phosphorylation provided by ETC to enhance apoptotic pathway. This fits with a recent report that showed that GAS can induce mitochondrial permeability leading to bioenergetic and redox damage. Thus evading host immune defense and causing inflammatory programmed cell death in monocytes (Goldmann 2009). Furthermore, it results have been recently reported that GAS streptolysin $\mathrm{S}$ and $\mathrm{O}$ virulence factors 
Figure 4-3. Pathway analysis of differentially expressed genes at 12 hrs post infection in peripheral blood of resistant and susceptible strains compared to uninfected (0hr) mice of respective strains.

Panel A. Venn diagram of differentially expressed genes in blood of resistant and susceptible strains, showing common (shared) and unique genes. Resistant strains had 380 genes differentially expressed within these, 268 genes were unique to resistant strains while 112 genes were shared with susceptible strains. A total of 650 genes were differentially expressed in susceptible strains within these genes, 538 genes were unique to susceptible strains. These lists of differentially expressed genes were parsed into pathways using ingenuity pathway analysis tool (IPA) as detailed in methods section. Shown are the most significant pathways. We parsed common (shared) differentially expressed genes in both resistant and susceptible strains into pathways shown in panel B. The significant pathways associated with genes that are differentially expressed only in resistant strains are shown in panel $\mathrm{C}$, while those associated only with susceptible strains are shown in panel D. Whether the genes within a pathway are up or down regulated is denoted in words and as arrows beside each pathway. The xaxis displays significance in (-log (p-value)) where the higher the number (the longer the blue bar) the more likely the association of the differentially expressed genes with the noted pathways is not by chance, this significance is also expressed as p-values on each bar. For each pathway we calculated a ratio (orange squares, and decimal number associated with it), the ratio is the number of differentially expressed genes divided by total number of molecules in the specified pathway. 
A Venn diagram of differentially expressed genes at $12 \mathrm{~h}$ B Pathways of differentially expressed genes shared (common) post infection in blood of resistant and susceptible strains, showing common (shared) and unique genes

\section{Differentially expressed genes in resistant(R) I susceptible(S) strains at $12 \mathrm{hrs}$ vs. uninfected $(\mathrm{Oh})$ mice}

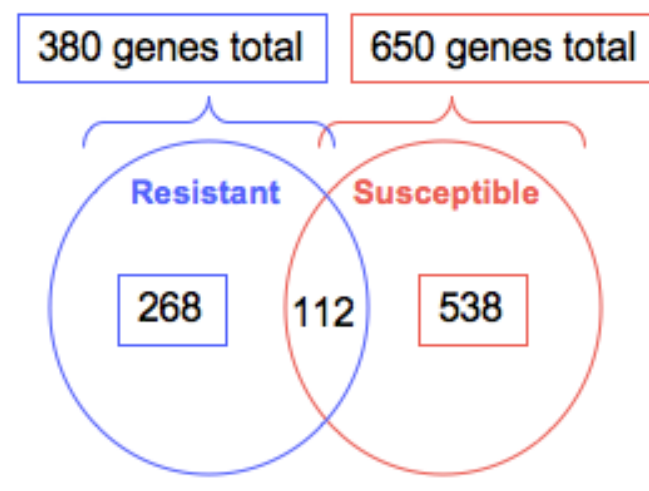

between resistant and susceptible strains at $12 \mathrm{~h}$ post infection

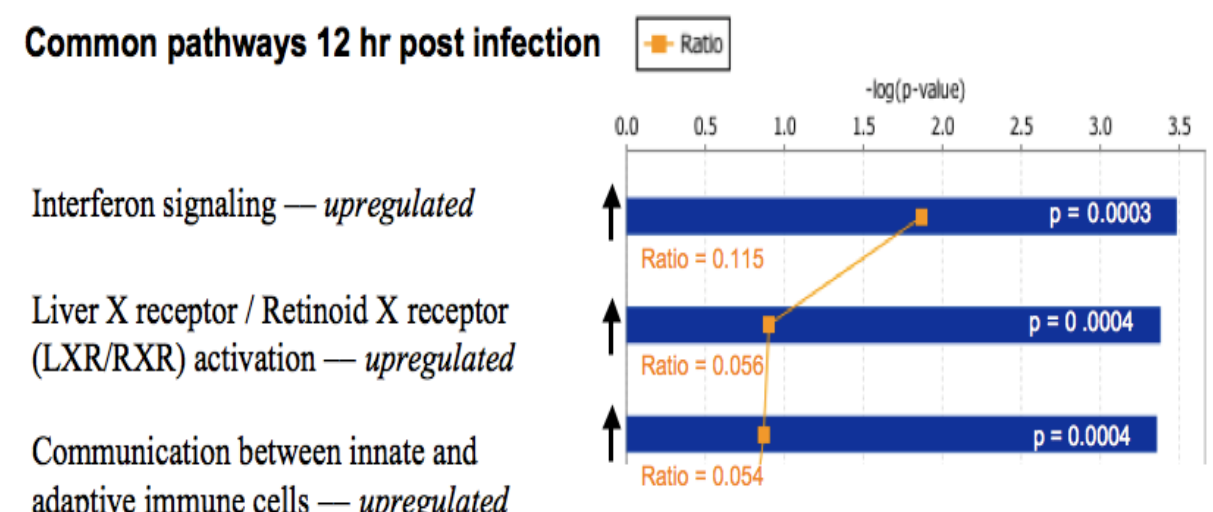


C Pathways of differentially expressed genes associated with resistant strains at $12 \mathrm{~h}$ post infection

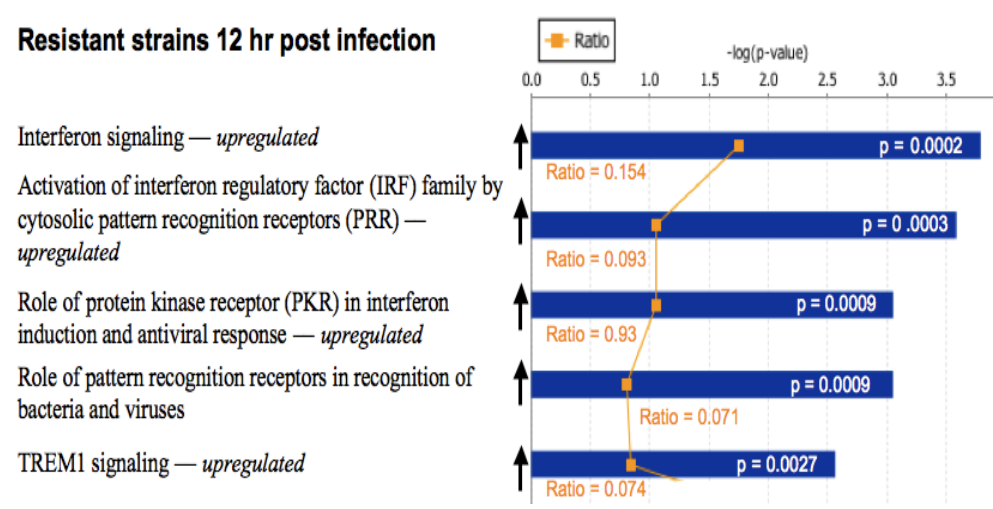

Figure 4-3. (Continued).
D Pathways of differentially expressed genes associated only with susceptible strains at $12 \mathrm{~h}$ post infection

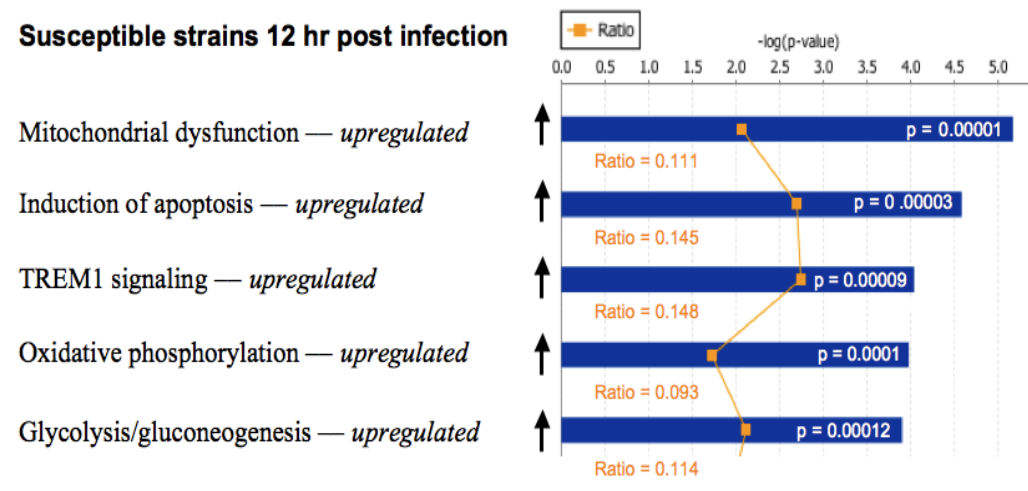


Table 4-2. Differentially expressed genes in peripheral blood of resistant and susceptible strains at 12 hrs post GAS infection.

\begin{tabular}{|c|c|c|c|}
\hline \multirow[t]{2}{*}{ Pathways and Genes/Transcripts } & \multirow[t]{2}{*}{ Gene/Transcript Description } & \multicolumn{2}{|c|}{ Fold Change } \\
\hline & & Susceptible & Resistant \\
\hline \multicolumn{4}{|c|}{$\begin{array}{l}\text { Top pathways associated with differentially } \\
\text { expressed genes shared between susceptible and } \\
\text { resistant strains } \\
\text { Interferon signaling and LXR/RXR signaling } \\
\text { pathways }\end{array}$} \\
\hline Ifit3 & $\begin{array}{l}\text { Interferon-induced protein with tetratricopeptide } \\
\text { repeats } 3 \text { (Ifit3) }\end{array}$ & 22.93 & 22.31 \\
\hline Tap1 & $\begin{array}{l}\text { Transporter 1, ATP-binding cassette, sub-family B } \\
\text { (MDR/TAP) }\end{array}$ & 4.21 & 2.78 \\
\hline Oas $1 \gamma$ & 2'-5' oligoadenylate synthetase $1 \mathrm{G}$ (Oas1 gamma) & 15.54 & 11.54 \\
\hline Ill 18 & Interleukin 18 & 6.84 & 5.03 \\
\hline Illrn & Interleukin 1 receptor antagonist, transcript variant 1 & 5.41 & 5.75 \\
\hline$N r 1 h 3$ & Nuclear receptor subfamily 1 , group $\mathrm{H}$, member 3 & 17.61 & 15.80 \\
\hline $\operatorname{Tlr} 4$ & Toll-like receptor 4 & 2.59 & 2.67 \\
\hline \multicolumn{4}{|c|}{$\begin{array}{l}\text { Top pathway associated with differentially } \\
\text { expressed genes in resistant strains (12h vs. 0h) } \\
\text { Interferon signaling pathway }\end{array}$} \\
\hline Stat1 & Signal transducer and activator of transcription 1 & & 4.00 \\
\hline Stat2 & Signal transducer and activator of transcription 2 & & 2.82 \\
\hline \multicolumn{4}{|c|}{$\begin{array}{l}\text { Top pathway associated with differentially } \\
\text { expressed genes in susceptible strains (12h vs. 0h) } \\
\text { Mitochondrial dysfunction }\end{array}$} \\
\hline App & Amyloid beta (A4) precursor protein & 8.51 & \\
\hline Casp8 & Caspase 8 , apoptosis-related cysteine peptidase & 2.84 & \\
\hline Cycl & Cytochrome c-1 & 2.61 & \\
\hline
\end{tabular}


Table 4-2 (Continued).

\begin{tabular}{|c|c|c|c|}
\hline \multirow[t]{2}{*}{ Pathways and Genes/Transcripts } & \multirow[t]{2}{*}{ Gene/Transcript Description } & \multicolumn{2}{|c|}{ Fold Change } \\
\hline & & Susceptible & Resistant \\
\hline Htra2 & HtrA serine peptidase 2 & 4.17 & \\
\hline Ndufa 8 & $\begin{array}{l}\text { NADH dehydrogenase (ubiquinone) } 1 \text { alpha } \\
\text { subcomplex } 8,19 \mathrm{kDa}\end{array}$ & 3.96 & \\
\hline Ndufa 9 & $\begin{array}{l}\text { NADH dehydrogenase (ubiquinone) } 1 \text { alpha } \\
\text { subcomplex } 9,39 \mathrm{kDa}\end{array}$ & 3.91 & \\
\hline Ndufs 2 & $\begin{array}{l}\text { NADH dehydrogenase (ubiquinone) Fe-S protein 2, } \\
49 \mathrm{kDa}\end{array}$ & 2.45 & \\
\hline$N d u f_{S} 3$ & $\begin{array}{l}\text { NADH dehydrogenase (ubiquinone) Fe-S protein } 3 \text {, } \\
30 \mathrm{kDa}\end{array}$ & 2.98 & \\
\hline$N d u f_{S} 7$ & $\begin{array}{l}\text { NADH dehydrogenase (ubiquinone) Fe-S protein } 7 \text {, } \\
20 \mathrm{kDa}\end{array}$ & 2.45 & \\
\hline Ndufs 8 & $\begin{array}{l}\text { NADH dehydrogenase (ubiquinone) Fe-S protein } 8 \text {, } \\
23 \mathrm{kDa}\end{array}$ & 2.76 & \\
\hline Sdhb & $\begin{array}{l}\text { Succinate dehydrogenase complex, subunit B, iron } \\
\text { sulfur (Ip) }\end{array}$ & 3.39 & \\
\hline Sdhc & Succinate dehydrogenase complex, subunit C & 2.24 & \\
\hline Uqcrcl & Ubiquinol-cytochrome $\mathrm{c}$ reductase core protein 1 & 2.92 & \\
\hline$X d h$ & Xanthine dehydrogenase & 6.20 & \\
\hline
\end{tabular}


specifically interfere with killing mechanisms by induction of mitochondrial dysfunction (Aikawa et al., 2010). Nonetheless, these previous studies did not elucidate the exact mechanisms and role of mitochondrial dysfunction in modulating severity of GAS sepsis. Therefore, more detailed studies with more time points focusing on the role of mitochondrial dysfunction in inducing multiple organ failure are required to further elucidate our results.

In general, our global gene expression studies comparing differential gene expression in blood of infected vs. uninfected susceptible and resistant strains at multiple times post infection gave us insight into some aspects of how the host might be modulating differential immune response to GAS sepsis. Our data suggested that the involvement of multiple pathways in modulating differential susceptibility, where starting as early as $8 \mathrm{~h}$ post infection, IL10 signaling pathways were upregulated only in resistant strains. In addition, pathways associated with bacterial killing and resolution of infection were triggered in resistant strains at that early stage. It was noteworthy that genes and pathways involved in macrophage and neutrophils killing mechanisms were also associated with these discovered pathways.

Next, we analyzed change in gene expression in spleen at 8,12,16, 24 and 36h post infection following the same design as blood microarrays studies detailed in Figure $4-1$. However, we chose to start by analyzing the $36 \mathrm{~h}$ post infection differential gene expression arrays, to investigate immune response after allowing enough time for the bacteria to disseminate to the spleen. Analysis of the differentially expressed genes in spleens of resistant and susceptible ARI-BXD strains revealed the engagement of the same pathways suggested by the systems genetics genome-wide association studies. These pathways encompass many of the genes located within all 3 survival QTLs, including the one on $\mathrm{Chr} \mathrm{X}$. In those studies, we infected susceptible and resistant ARIBXD strains with M1T1 GAS, uninfected control mice from the same strains were injected with saline. The data revealed significant changes in the expression of highly relevant genes in both susceptible and resistant mice, both pre- and post infection (Figure 4-4). Many of those genes are located within our 3 mapped QTLs (e.g. Il1 $\alpha$, Il1 $\beta$, and Il1 rn) on Chr 2, in addition to Irf1 on Chr X. These genes and pathways are likely to contribute to the pathogenesis and differential severity of GAS sepsis. However, this spleen microarray data is very preliminary, with further analyses and more samples needed to be done for obtaining a clearer picture of mechanisms of differential susceptibility.

\section{Soluble Biomarkers Associated with Differential Susceptibility in Select Susceptible and Resistant Strains at Multiple Time Series Post-infection}

Next, we wanted to dissect soluble biomarkers associated with differential response. Therefore, we aimed to analyze the kinetics of selected cytokines levels in plasma of uninfected vs. infected resistant and susceptible strains at selected time points post infection. We analyzed differences in the levels of selected cytokines that are known to be associated with modulation of differential severity of GAS sepsis and were associated with differential gene expression analyses detailed in the first section of 


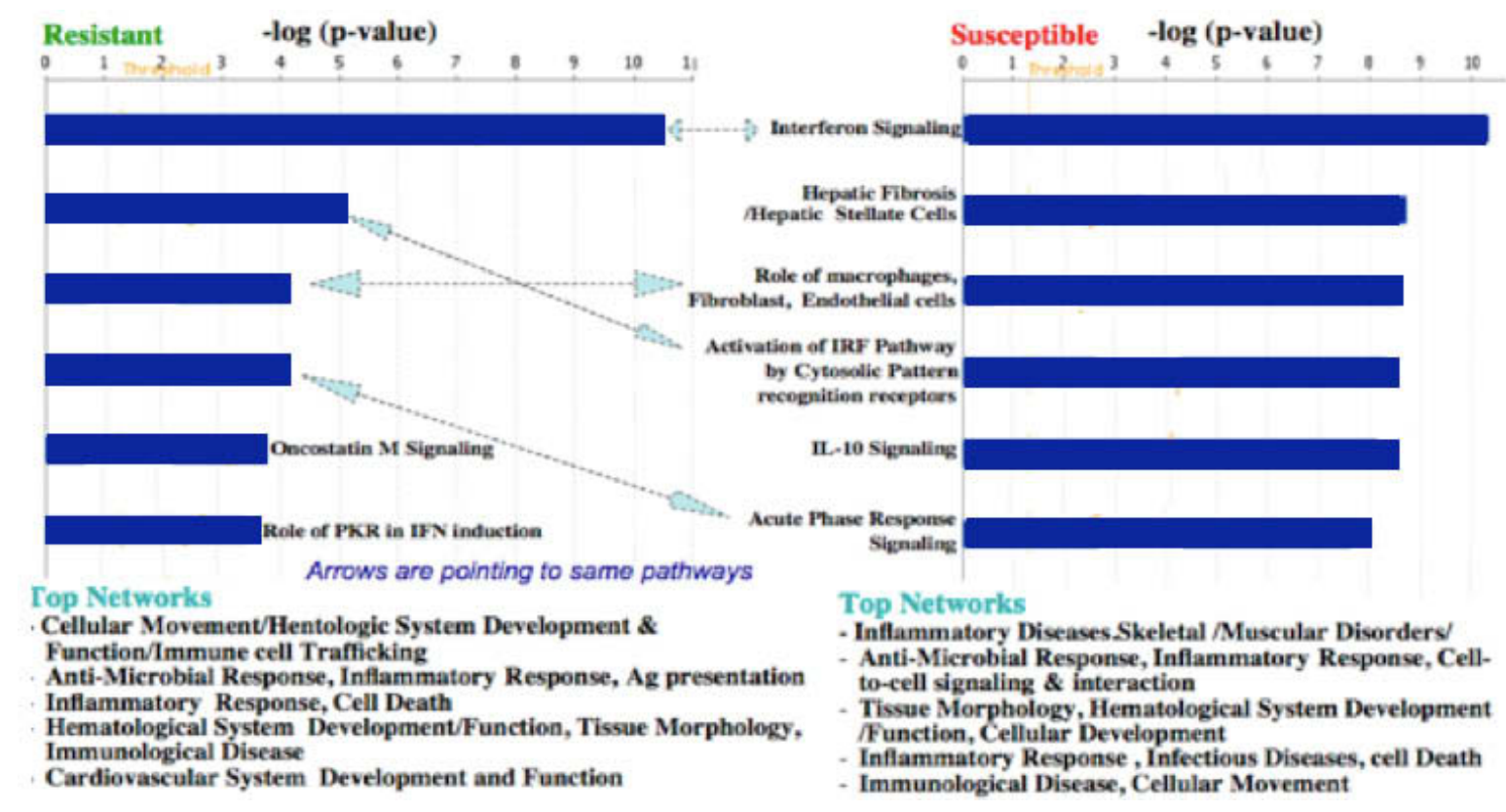

\section{Figure 4-4. Pathways associated with differentially expressed genes in spleens of uninfected versus infected resistant and susceptible ARI-BXD strains at 36h post GAS infection.*}

We infected selected highly susceptible and highly resistant ARI-BXD strains $(n=3-4$ strains in each group, 3-5 mice per strain per time point) with M1T1 GAS strain. In parallel, a control group of mice was injected with saline. Differentially expressed genes in spleen at $36 \mathrm{~h}$ post infection were parsed into pathways, shown are the most significant pathways in resistant and susceptible group. The $\mathrm{x}$-axis displays significance in $(-\log (\mathrm{P}$-value $))$ where the higher the number the more likely the association is not by chance. Matching pathways shown were connected with arrows in light blue.

\footnotetext{
* Data represented in this Figure is preliminary, as we are planning to do more analysis aswell as more time points that we have already collected samples, these samples represent earlier stages of infection. We are continuing to analyze these data to elucidate mechanisms associated with differential response.
} 
Chapter 4. We investigated selected pro-inflammatory cytokines, interleukin 6 (IL-6), interferon gamma (IFN $\gamma$ ) and tumor necrosis factor alpha (TNF $\alpha)$ and anti-inflammatory cytokine, IL10 at selected time points post-infection $(0,8,12$ and 24 hours).

We first aimed to analyze stage specific differences within each group, i.e. susceptible group at different time points post infection and similarly resistant group. We found that in susceptible strains TNF $\alpha$ showed significant increase in plasma levels at 8 hours post infection, followed by a drop in plasma levels at 12 hours to almost the same levels as "zero" or uninfected control mice, then no changes at $24 \mathrm{~h}$ from $12 \mathrm{~h}$ (Figure 4-5-A) ${ }^{*}$. This might suggest that there might be a possible early induction of acute systemic sepsis manifested by the observed high systemic levels of TNF $\alpha$ at 8 hours in susceptible strains only. As for the resistant strains studied, we found that levels of TNF $\alpha$ significantly increase in the plasma only at $24 \mathrm{~h}$ post infection (compared to uninfected mice) (Figure 4-5-B). This might suggest that the release of TNF $\alpha$ at that stage of infection is more beneficial and induces appropriate response to resolve infection as these strains are protected against sepsis. However, on comparing TNF $\alpha$ levels in resistant vs. susceptible group at each of studied stages of infection, there were trends of differences but none reached significant (Figure 4-5-C).

For IL6 both susceptible and resistant strains showed significant increase in plasma levels at 12 and 24 hours post infection in comparison to $0 \mathrm{~h}$ (uninfected controls) (Figure 4-6-A and 4-6-B). However, the levels of IL6 in susceptible strains at 24 hours post infection were almost double that of resistant strains, yet it didn't reach statistical significance differences due to the spread of data points (Figure 4-6- C). Collectively, TNF $\alpha$ and IL6 pro-inflammatory cytokines and crucial mediators of systemic sepsis didn't show differences in the levels between susceptible and resistant strains. However, we realize that this might due to assessing plasma levels of these cytokines, which does not reflect the actual producing cells; therefore, we plan to perform intracellular cytokine staining, where we can determine which cells are producing theses cytokines at the studied stages of infection and hence draw a more definitive conclusion. Yet, our results indicate that at these early time points, systemic levels of IL6 and TNF $\alpha$ do not differ between susceptible and resistant strains at the selected time points.

Next we looked at levels of anti-inflammatory cytokine IL10 in susceptible and resistant strains at multiple stages of infection. We found that both susceptible and resistant strains showed significantly high levels at 8 and 12 hours post-infection (Figure 4-7-A and 4-7-B). However, levels of IL10 were 2-3 folds higher in resistant strains than susceptible strains; indeed comparing susceptible to resistant strains, showed that higher levels of IL10 were associated with resistant strains at as early as 8 and 12 hours post

\footnotetext{
* The analyzed data shown in Figures 4-5 to 4-9 are representive of susceptible and resistant groups, where 2 mice per strain per time point were analyzed, with the exception of:a) susceptible group, where we used 4 mice at $8 \mathrm{~h}$ and 12h for BXD 97 and for BXD77 we used 4 and 6 mice at 12 and $24 \mathrm{~h}$ respectively b) Resistant group, we used 4 mice at 8,12 and $24 \mathrm{~h}$ of BXD87 and one mouse at 8 hours of BXD48. We collected far more plasna samples that are to be assayed. All other data shown is for 2 mice per strain ( 3 strains used) per time point ( 4 time points analyzed).
} 
Figure 4-5. TNF $\alpha$ plasma levels profiles across early time points post infection comparing selected highly susceptible and highly resistant ARI-BXD strains.

We infected three highly susceptible ARI-BXD strains (BXD100, 77 and 97) and three highly resistant ARI-BXD strains (BXD48, 73 and 87) with 2 X $10^{7}$

CFU/mouse of hypervirulent M1T1 GAS strain, design of study is similar to microarray studies shown in Figure 4-1, further details of cytokine measurements in methods section. We sacrificed and collected plasma for a set of mice per strain at each time point post infection, 8,12 , and 24 hours, therefore, there were no multiple bleedings of any of the mice used. In parallel, a control group of uninfected mice were used for collection of plasma for estimating normal levels of TNF $\alpha$ in these selected strains. (A). Comparison of medians of levels of TNF $\alpha$ in susceptible mice showed that levels of TNF $\alpha$ increased significantly at 8 hours post-infection, then significantly drop back to almost the zero levels at 12 hours post infection. Panel (B). Resistant strains showed significant increase in levels of TNF $\alpha$ only at 24 hours postinfection. Panel C. We compared TNFa plasma levels in susceptible (red dots) vs. resistant (blue dots) groups at the analyzed time points. There were differences in levels of TNF $\alpha$ but none reached statistical significance based on Mann-Whitney test. We applied Kruskal-wallis test to assess statistical significance of differences in levels of TNF $\alpha$ between and across time points (stages of infection) (panels A and B). In general a $p$ value of $<0.05$ was considered significant. 
A Susceptible strains

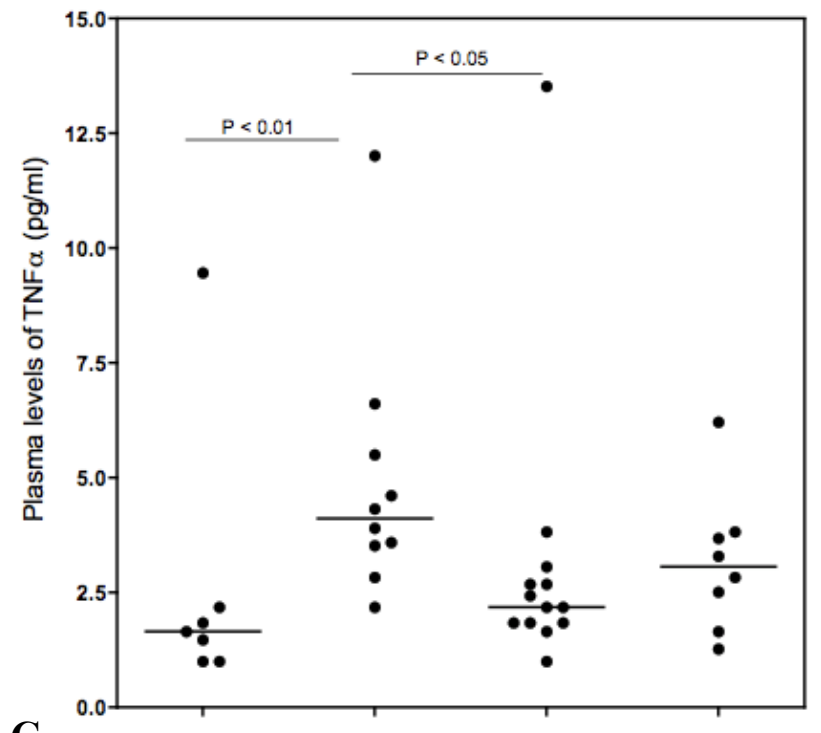

C

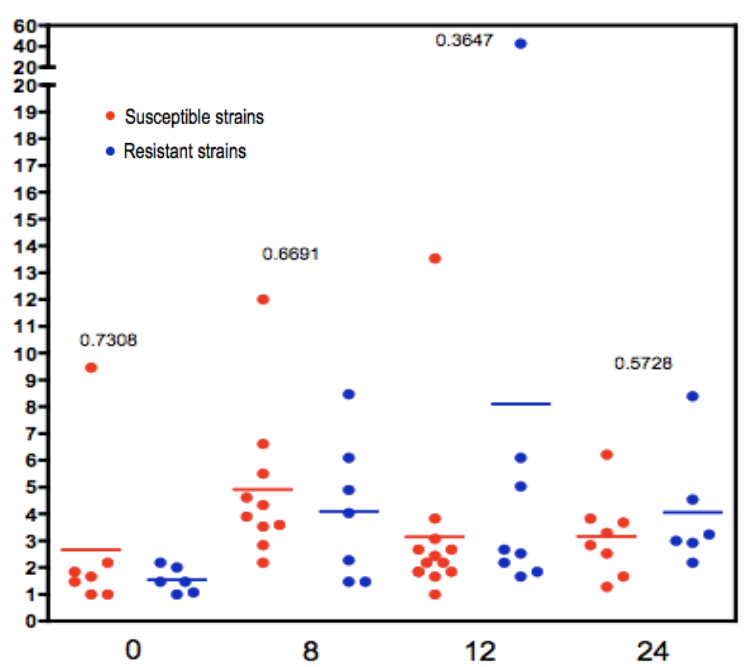

\section{B Resistant strains}

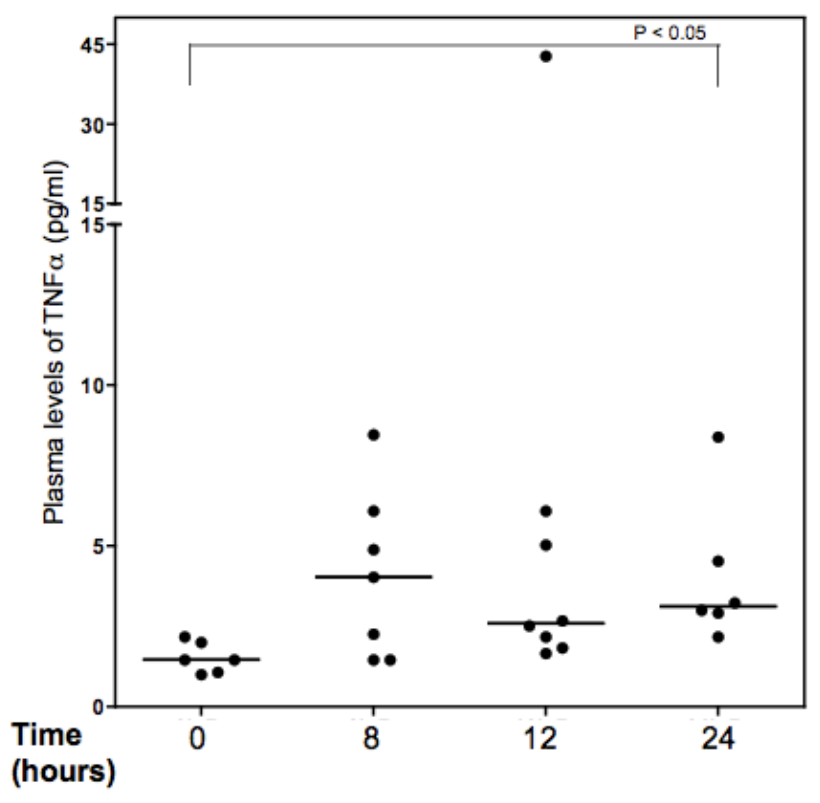


Figure 4-6. IL6 plasma levels profiles across early time points post infection comparing selected highly susceptible and highly resistant ARI-BXD strains.

We infected three highly susceptible ARI-BXD strains (BXD100, 77 and 97) and three highly resistant ARI-BXD strains (BXD48, 73 and 87) with 2 X $10^{7} \mathrm{CFU} /$ mouse of hypervirulent M1T1 GAS strain We sacrificed and collected plasma for a set of mice per strain at each time point post infection, 8,12 , and 24 hours, therefore, there were no multiple bleedings of any of the mice used. In parallel, a control group of uninfected mice were used for collection of plasma for estimating normal levels of IL6 in these selected strains. (A). Comparison of medians of levels of IL6 in susceptible mice showed that levels of IL6 increased significantly at 12 and 24 hours postinfection compared to pre-infection (zero hour). Panel B. Similarly, resistant strains showed significant increase in levels of IL6 12 and 24 hours post-infection, however, the maximum value and hence the range of IL6 levels was higher and wider in susceptible strains, up to $3500 \mathrm{pg} / \mathrm{ml}$ vs. $1800 \mathrm{pg} / \mathrm{ml}$ in resistant strains. Panel C. We compared IL6 plasma levels in susceptible (red dots) vs. resistant (blue dots) groups at the analyzed time points. There were differences in levels of IL6 but none reached statistical significance based on Mann-Whitney test. We applied Kruskal-wallis test to assess statistical significance of differences in levels of IL6 between and across time points (stages of infection) (panels A and B). In general a p-value of $<0.05$ was considered significant. (For number of samples analyzed please refer to page 65 footnote). 
A Susceptible strains

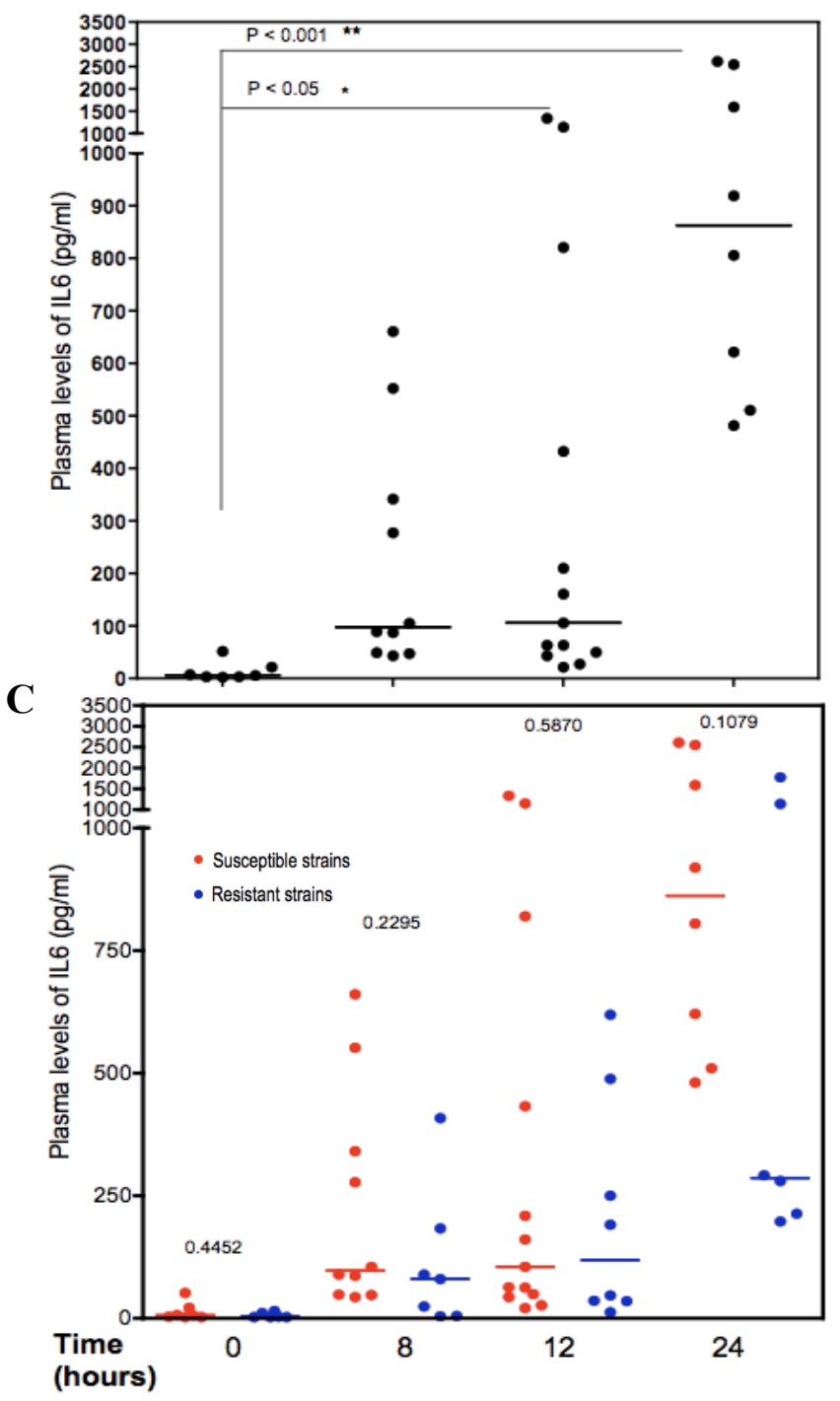

B Resistant strains

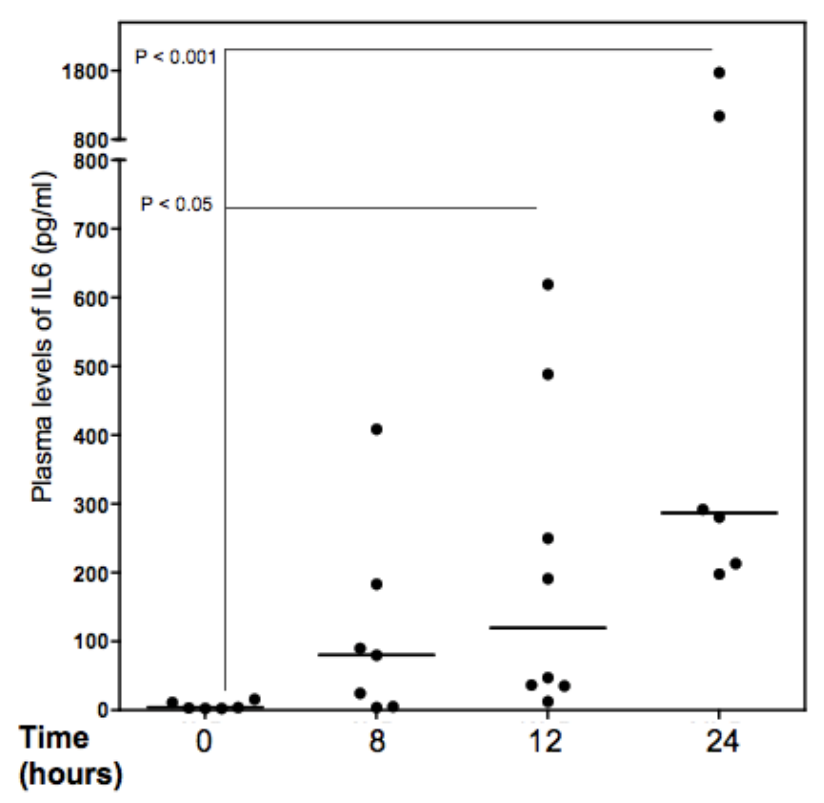


Figure 4-7. IL10 plasma levels profiles across early time points post infection comparing selected highly susceptible and highly resistant ARI-BXD strains.

We infected three highly susceptible ARI-BXD strains (BXD100, 77 and 97) and three highly resistant ARI-BXD strains (BXD48, 73 and 87) with 2 X $10^{7} \mathrm{CFU} /$ mouse of hypervirulent M1T1 GAS strain We sacrificed and collected plasma for a set of mice per strain at each time point post infection, 8,12 , and 24 hours, therefore, there were no multiple bleedings of any of the mice used. In parallel, a control group of uninfected mice were used for collection of plasma for estimating normal levels of IL10 in these selected strains. (A). Comparison of medians of levels of IL10 in susceptible mice showed that levels of IL10 increased significantly at 8 hours post-infection compared to pre-infection (zero hour), then dropped at 12 and 24 hours with significant drop at 24 to almost same as "zero" hour. Panel B. Similarly, resistant strains showed significant increase in levels of IL10 at 8 hours post infection, with a drop at 12 and 24 hours postinfection, however, levels at 12 hours were still significantly higher than zero hour. In addition, in general the resistant strains had higher levels of IL10 levels than the susceptible strains. Panel C. We compared IL10 plasma levels in susceptible (red dots) vs. resistant (blue dots) groups at the selected analyzed time points. We found that IL10 levels were significantly higher in plasma of resistant strains at early time points (8 and 12 hours) however, at 24 hours post infection both susceptible and resistant strains IL10 levels dropped and no statistical differences between their levels. Significance between susceptible and resistant strains IL10 levels were calculated based on Mann-Whitney test. We applied Kruskal-wallis test to assess statistical significance of differences in levels of IL10 between and across time points (stages of infection) (panels A and B). In general a p-value of $<0.05$ was considered significant. (For number of samples analyzed please refer to page 65 footnote). 
A Susceptible strains

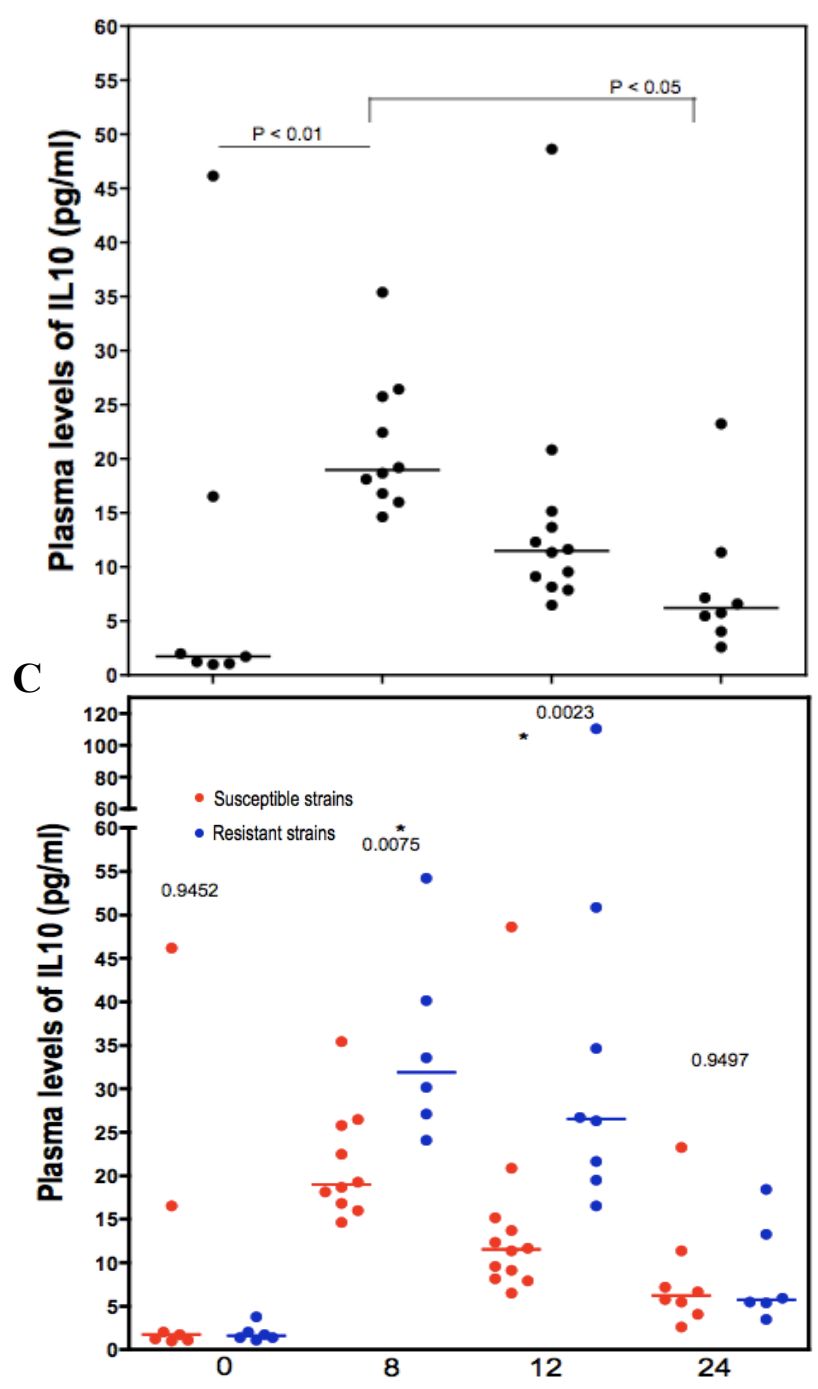

B Resistant strains

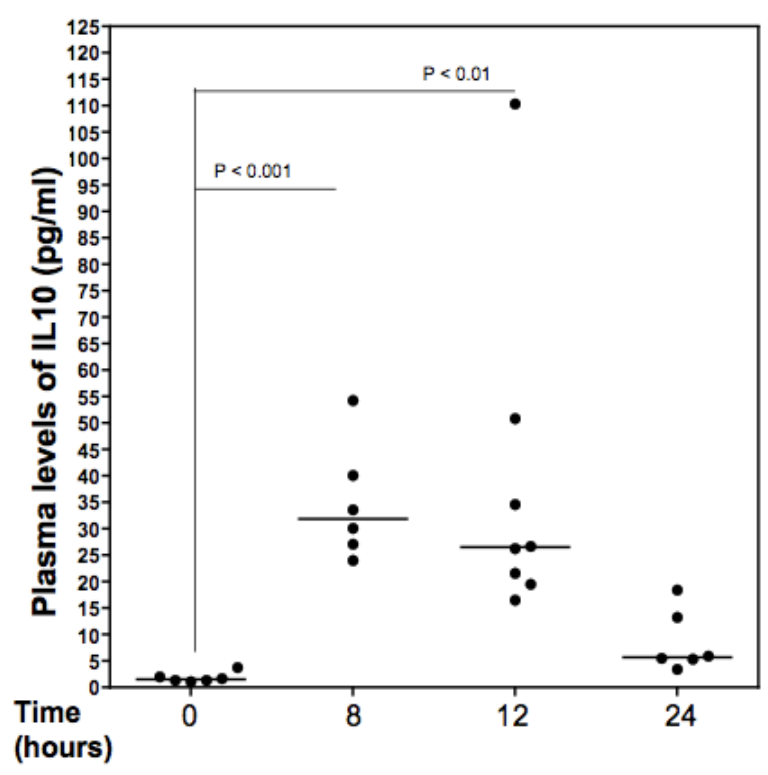


infection (Figure 4-7-C). The association of higher levels of anti-inflammatory IL10 suggested that an active immunosuppressive of the cytokine avalanche might be the mechanism of resistance and protection against sepsis. While highly susceptible strains had lower levels of IL10 suggesting an unchecked cytokine response was associated with higher severity of sepsis.

IFN $\gamma$ was significantly higher in susceptible strains at 24 hours post infection; however, in resistant strains there were no significant increase in levels of IFN $\gamma$ (Figure 4-8-A and 4-8-B). Comparing levels of IFN $\gamma$ resistant to susceptible strains, again 24 hours susceptible strains had higher levels, this being a biologically relevant time point for IFN $\gamma$ actions. (Figure 4-8-C) Collectively, results from pro-inflammatory cytokines, IL6, TNF $\alpha$ and IFN $\gamma$, showed increase in levels in susceptible across time points suggested that systemic acute response in susceptible strains. However, these increase were also seen in resistant strains (Figures 4-5, 4-6, and 4-8 panels A and B), yet we postulated that the increase in these pro-inflammatory cytokines was neutralized or modulated by anti-inflammatory cytokines in resistant strains. Therefore, we compared anti- to pro- inflammatory cytokines ratios, as shown in Figure 4-9 comparing IL10/IFN $\gamma$ ratios. This comparison reveled that resistant strains had higher levels of IL10/IFN $\gamma$ ratio at all time points post infection and even at $0 \mathrm{~h}$ prior to infection. Collectively, our data suggest that susceptibility to severe GAS sepsis is mainly associated with unchecked production of pro-inflammatory cytokines and mediators at early time points. Meanwhile, protected mice are better able to down regulate excessive inflammatory responses and hence are protected against STSS. Our cytokine profiling analyses validated the pathways discovered through our transcriptome profiling of blood cells from the mice at different time points post infection in addition to our GWAS mapping results.

Moreover, our findings outlined in chapters 3 and 4 of this dissertation are in concordance to the clinical disease data; where our recent human ex vivo data showed that IL10 exerts active suppression of exaggerated immune responses in individuals carrying protective HLA-II alleles (DR15/DQ6) (Nooh and Kotb in press). Whereas individuals carrying the STSS high-risk alleles (DR14/DQ5) mounted significantly lower levels of IL10 and higher levels of IFN $\gamma$ (Nooh and Kotb in press).Further validation was done by the addition of exogenous rIL10 to PBMCs isolated from individuals carrying high-risk HLA-II alleles (DR14/DQ5) decreased SAg-induced T cell proliferation and cytokine responses (Nooh and Kotb in press). Moreover, the association of higher levels of IL10 with protection from sepsis was also observed in our human transcriptome profiles of patients with severe vs. non-severe GAS sepsis (Kanasal and Kotb in final preparation).

Collectively, our murine in vivo and in vitro data with our human ex vivo data support our hypothesis that resistance (protection) is actively exerted by the production of the anti-inflammatory immunomodulatory IL10. In addition, IL10 is highly regulated and can be produced by a number of innate immune cells, including dendritic cells, macrophages, and most recently it has been shown to be produced by neutrophils (Saraiva and O'Garra, 2010).We observed that IL10 in plasma was detected at a rather early stage of the infection ( 8 and 12). Therefore, it is likely that innate immune cells are producing IL10. Interestingly, recent reports indicated that neutrophils can exert 
Figure 4-8. IFN $\gamma$ plasma levels profiles across early time points post infection comparing selected highly susceptible and highly resistant ARI-BXD strains.

We infected three highly susceptible ARI-BXD strains (BXD100, 77 and 97) and three highly resistant ARI-BXD strains (BXD48, 73 and 87) with 2 X $10^{7} \mathrm{CFU} /$ mouse of hypervirulent M1T1 GAS strain We sacrificed and collected plasma for a set of mice per strain at each time point post infection, 8,12 , and 24 hours, therefore, there were no multiple bleedings of any of the mice used. In parallel, a control group of uninfected mice were used for collection of plasma for estimating normal levels of IFN $\gamma$ in these selected strains. (A). Comparison of medians of levels of IFN $\gamma$ in susceptible mice showed that levels of IFN $\gamma$ increased significantly only at 24 hours post-infection compared to pre-infection (zero hour). In addition, levels at 24 hours were significantly higher than 12 hours post infection Panel B. Resistant strains showed increase in levels of IFN $\gamma$ post-infection, however, none reached statistical significance. Panel C. We compared IFN $\gamma$ plasma levels in susceptible (red dots) vs. resistant (blue dots) groups at the analyzed time points. There were significant differences in levels of IFN $\gamma$ between resistant and susceptible strains only at 24 hours, which is the biologically relevant time point for IFN $\gamma$ actions. Statistical significance comparing resistant to susceptible groups was based on Mann-Whitney test. We applied Kruskal-wallis test to assess statistical significance of differences in levels of IFN $\gamma$ between and across time points (stages of infection) analyzed (panels A and B). In general a p-value of $<0.05$ was considered significant. (For number of samples analyzed please refer to page 65 footnote). 
A Susceptible strains

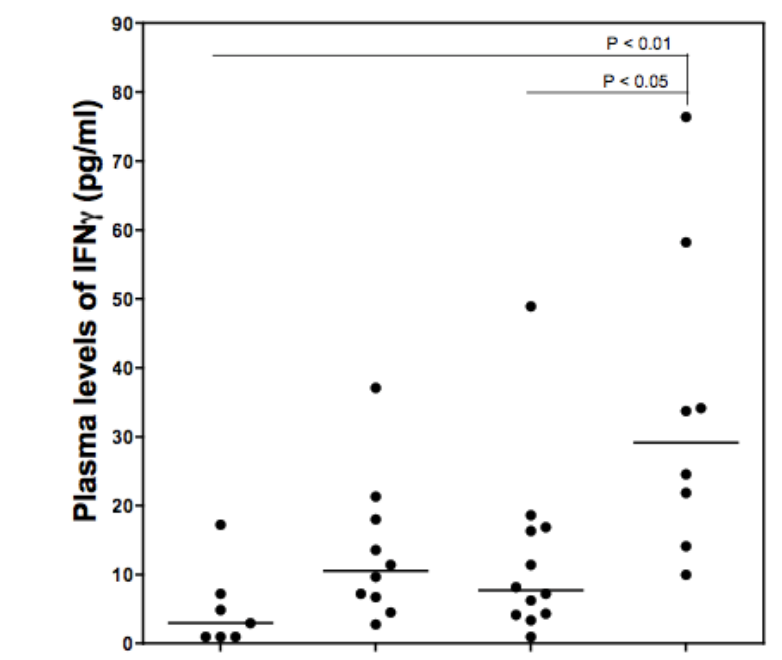

C

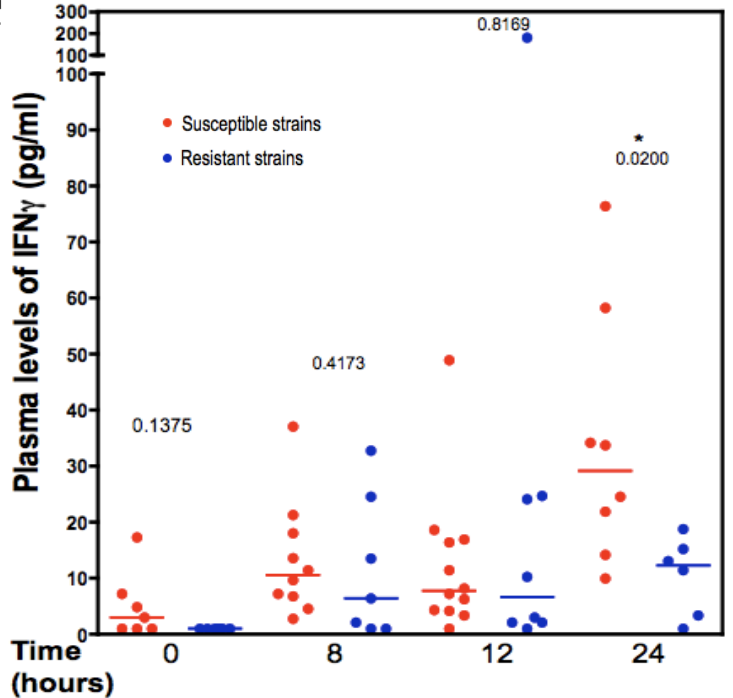

B Resistant strains

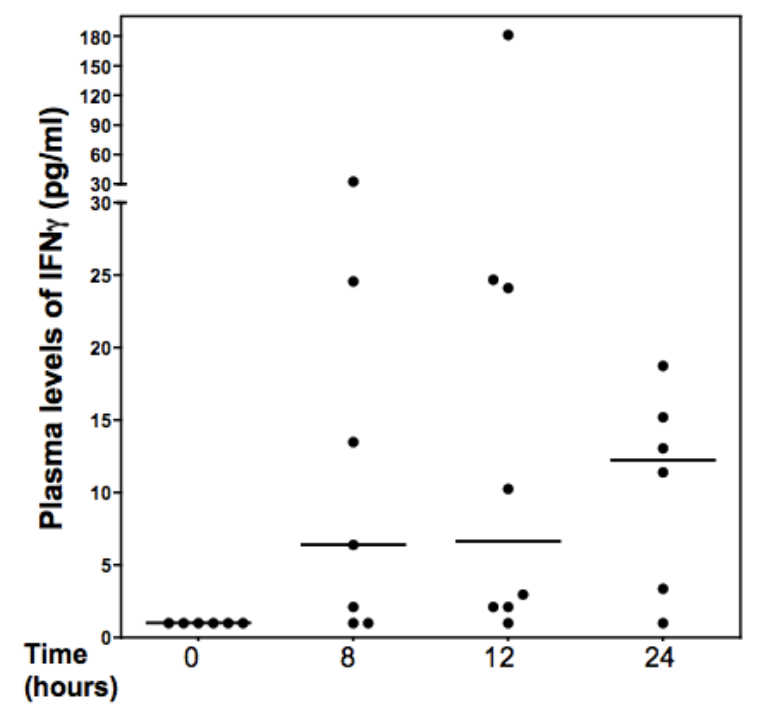


Figure 4-9. Comparison of ratios of anti-inflammatory to pro-inflammatory cytokines, comparing ratios of medians of IL 10/IFN $\gamma$ plasma levels across early time points post infection in selected highly susceptible and highly resistant ARI-BXD strains.

We infected three highly susceptible ARI-BXD strains (BXD100, 77 and 97) and three highly resistant ARI-BXD strains (BXD48, 73 and 87) with 2 X $10^{7} \mathrm{CFU} /$ mouse of hypervirulent M1T1 GAS strain We sacrificed and collected plasma for a set of mice per strain at each time point post infection, 8, 12, and 24 hours, therefore, there were no multiple bleedings of any of the mice used. In parallel, a control group of uninfected mice were used for collection of plasma for estimating normal levels of IL10 and IFN $\gamma$ in these selected strains. Data for individual cytokines were shown in Figures 4-7 and 48. Shown here are data from Figures 4-7 and 4-8 reanalyzed where medians of each cytokine at each time point was calculated and ratio of medians of IL10/IFN $\gamma$ was calculated. Resistant group showed higher ratio of IL10/IFN $\gamma$ than susceptible group at all the compared time points, indicating that anti-inflammatory levels are governing the immune response in resistant strains, adjusting the pro-inflammatory levels to appropriate levels and keeping the pro-inflammatory cytokines in check. Higher levels of IL10 were generally associated with protection, where we postulate that uncontrolled pro-inflammatory cytokine avalanche leads to sepsis in the current studied GAS sepsis model. (For number of samples analyzed please refer to matierials and methods section). 


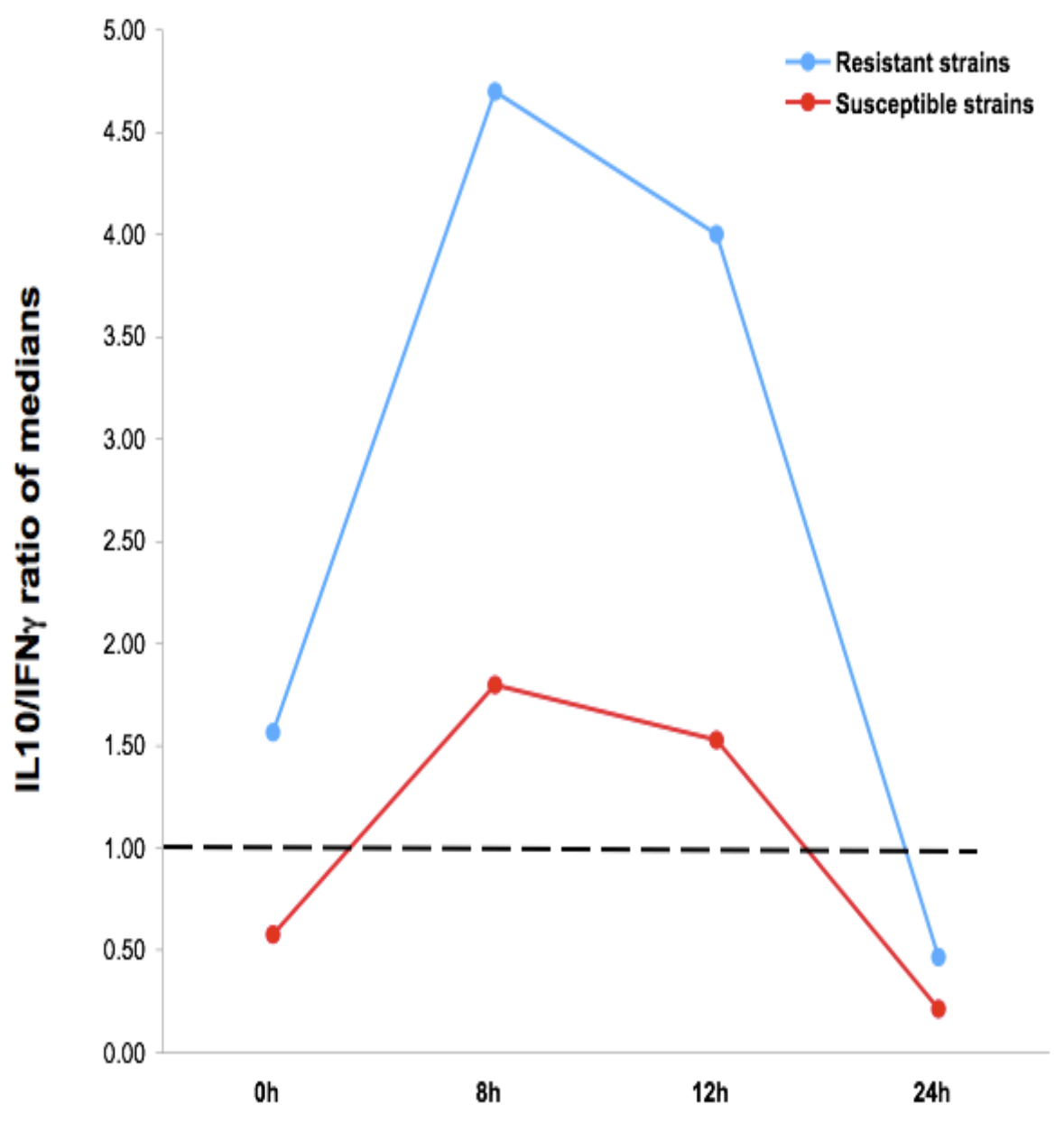


immunomodulatory effects by secreting IL10 (Cassatella et al., 2009; Zhang et al., 2009b). These findings are interesting as it agrees with our hypothesis of IL10 exerting active suppression of exaggerated immune responses and hence high levels of IL10 are associated with protection. More detailed studies to support or refute this hypothesis are needed, with further dissection of the causal agent and modulator of IL10 levels, which we hypothesized to be PGE2 as discussed in the next section. In conclusion, the association of GAS sepsis severity with cytokines exerting their effects temporally underscores the complexity of traits modulating severe GAS sepsis.

\section{Analysis of the Role of Prostaglandin at Early Stage of Infection}

The QTLs we mapped to Chr 2 have several immune-related genes that are polymorphic and made biological sense. We narrowed down our QTLs using multiple bioinformatics tools including linkage analyses, gene ontology analyses, and polymorphism analyses; at this point we had a candidate gene list that we parsed into pathways. The resultant pathways were validated by selecting key genes in each pathway and validating differential levels in susceptible and resistant strains post infection (as detailed in chapter 3). Real time PCR analyses associated differential susceptibility with two main pathways, IL1 and prostaglandin E pathways (Abdeltawab et al. 2008 and chapter 3 in this dissertation). In this section of the dissertation, I am going to focus on studies we performed to find prostaglandins association with differential susceptibility to GAS sepsis.

There are a total of five prostaglandins genes located either within our two main mapped QTLs on Chr 2 or located in their proximity. These genes were differentially expressed in our quantitative PCR analyses, however, we didn't detect these differences in our array data at the time points we selected. The five prostaglandin genes in our QTL include, prostaglandin D2 synthase (Ptgds), Chr $2(25.28 \mathrm{Mb})$, which is a polymorphic (see Appendix C) gene encoding a lipocalin-type prostaglandin. Over expression of Ptgds in lung was associated with better outcomes in bacterial pneumonia model (Joo et al., 2007). Two other prostaglandins are located in the proximity of mapped QTLs, prostaglandin-endoperoxide synthase 1 (Cox-1) (Chr $2(36.05 \mathrm{Mb}))$ and prostaglandin I2 (Ptgis) aka prostacyclin (Chr $2(166.89 \mathrm{Mb}))$. Prostacyclin has immunoregulatory properties similar to PGE2, with immunosuppressive actions on macrophage in infectious diseases (Aronoff et al., 2007). Of particular interest are prostaglandin E synthases 1 (Ptges) and 2 (Ptges2), on $\mathrm{Chr} 2$, at 30.71 and $32.21 \mathrm{Mb}$, respectively. These two genes were quite interesting as we found that increased expression levels of Ptges and Ptges 2 were associated with increased susceptibility to GAS sepsis (chapter 3 in this dissertation and (Abdeltawab et al., 2008). Although following Occam's razor principle one would postulate that one gene within these QTLs might be the modulator of the differential susceptibility observed. In addition, it might be that the co-existence of these immunerelated genes is by chance since the QTL is huge ( $10 \mathrm{Mb}$ in size) allowing co-existence of multiple immune-related genes. However, we found that these candidate genes were polymorphic (Appendix C), in addition, pathway analyses revealed that these mapped genes act together, moreover, our QTL acts as cis-acting QTL. 
Based on our QTL analyses and our quantitative gene expression analyses and literature associated with other infectious diseases, we hypothesized that exaggerated production of prostaglandins E2 (PGE2), the product of PTGES, in susceptible strains might predispose them to severe response to GAS. Meanwhile, controlled amounts of PGE2 in resistant strains could modulate a balanced response. To test our hypothesis, we aimed to quantify PGE2 levels in susceptible and resistant strains at early time points post infection that are more relevant to the quick responses mounted by PGE2. Being a lipid mediator, PGE2 can exert its actions within few minutes post infection and is crucial in the first 24 hours of infection (reviewed in [Harris et al., 2002; Bos et al., 2004; Sakata et al., 2010]). Therefore, we investigated the levels of PGE2 at 30 minutes intervals after infecting susceptible and resistant strains with the hypervirulent M1T1 GAS strain. We sacrificed 4 mice per strain at each time point, examining the first 2 hours in 30 minutes intervals (30, 60, 90 and 120 minutes) following infection (Figure 4-10). We found that susceptible strains had significantly higher PGE2 levels compared to resistant mice, especially during the first 60 min post-infection (Figure 4-10). This suggested that our hypothesis is valid and matched our real time PCR expression data of Ptges and Ptges2, our mapped QTL, and the association between increased levels of PGE2 and severe of GAS sepsis. Further studies to elucidate the mechanism of action are undergone in the laboratory.

Our finding that higher levels of PGE2 association with severe GAS sepsis along with our finding its synthases in our mapped QTL in our GWAS (Chapter 3, Appendix A and Abdeltawab et al. 2008). These synthases are polymorphic (Appendix C) and their levels are differential expressed as observed in our qPCR results (Chapter 3 and Abdeltawab et al. 2008). Collectively this led us to believe that PGE2 is an excellent candidate modulator of differential susceptibility, however, the mechanism of modulation needs further studies especially that PGE2 mechanism of action is complicated by the fact that it acts as a Janus mediator. PGE2 can act as pro-inflammatory mediator inducing IL6 production and causing vasodilatation and vascular leakage in inflammatory responses (reviewed in [Harris et al., 2002; Bos et al., 2004; Sakata et al., 2010]). Both events are crucial in GAS sepsis severity. In addition, PGE2 can act as an immunosuppressive, reducing neutrophil migration and bacterial killing by macrophages, by blocking production of reactive oxygen intermediates (ROI) (Sakata et al. 2010).

Moreover, it has been debated whether PGE2 promotes Th1 or Th2 responses, however, recent studies show that PGE2 activates its EP2 and EP4 receptors, and promotes Th1 differentiation and Th17 expansion (Yao et al., 2009)However, the two faces of PGE2 are not mutually exclusive, as PGE2 actions depend on multiple factors, including the cells it is exerting its actions on, stage of infection, receptors being activated and nature of the activation signal, and more importantly the concentration of PGE2. Where studies have shown that if the fine balance of this mediator is disturbed it can lead to pathological consequences. For example alterations in the amounts of PGE2 are associated with high risk of infection with HIV, cancer, and malnutrition (Lima et al., 2006; Anstead et al., 2009; Greenhough et al., 2009). 


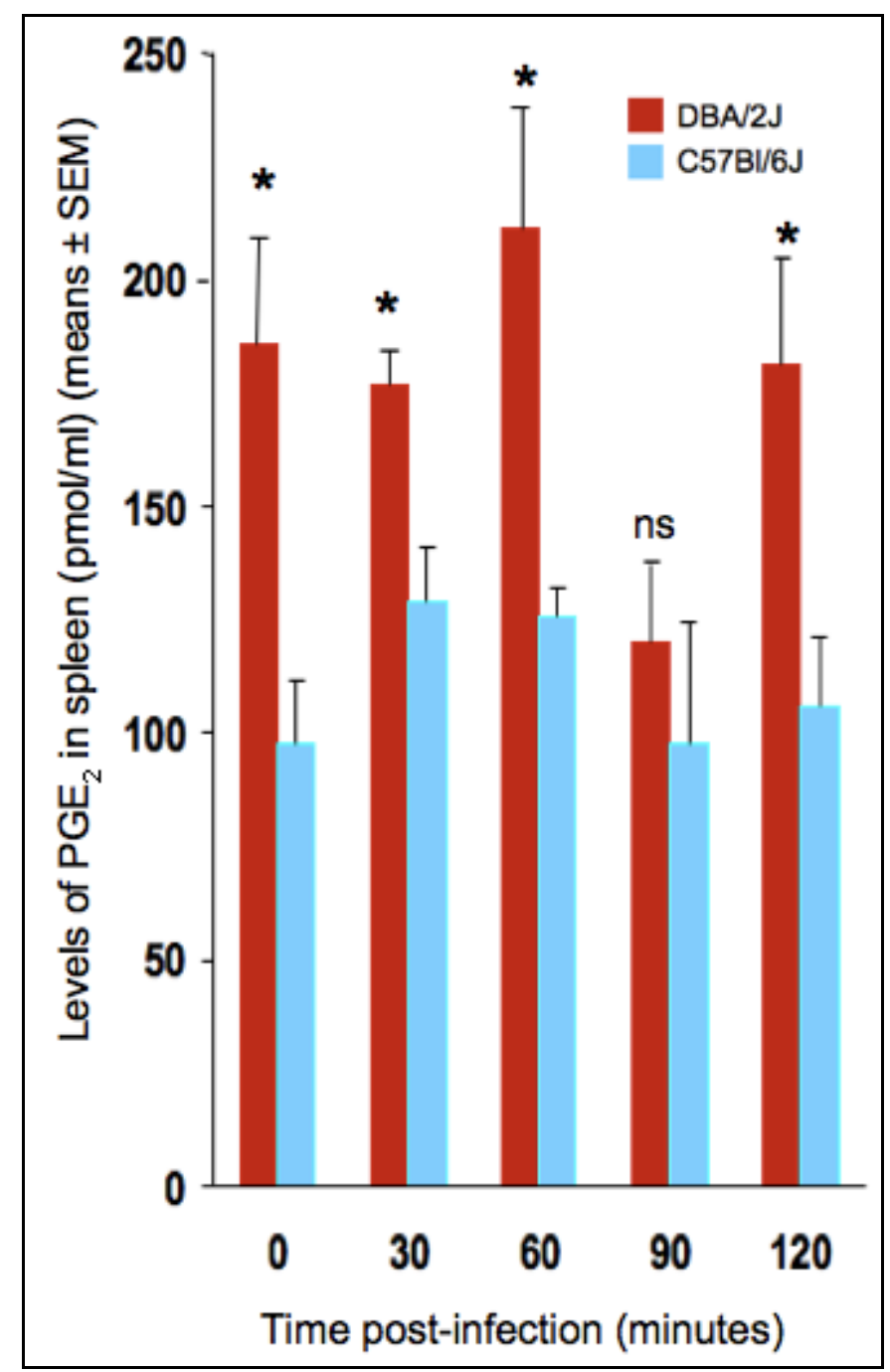

Figure 4-10. Analysis of prostaglandin role at early stages of severe GAS sepsis.

Higher levels of PGE2 in susceptible strains at all examined stages of infection were observed. We used the parental strains of BXD panel, C57Bl/6J (B6) and DBA/2J (D2), ( $\mathrm{n}=4$ mice per strain per time point). The time points examined were, 0, 30, 60, 90 and 120 minutes post infection. Data shows that D2 (the susceptible parental strain) has higher levels of PGE2 compared to B6 even before infection (0hr). Following infection with GAS (2X10 $\mathrm{CFU})$, D2 PGE2 levels remained high throughout the observed 120 min following infection except for a drop at $90 \mathrm{~min}$. Meanwhile, B6 seemed to maintain a steady level of PGE2 with a trend of slight increase at 30-60 min then back to starting levels at 90-120 min. 
Excitingly, studies have shown that PGE2 can inhibit production of IL10 from T cells, and to exacerbate Th17 mediated inflammation (Boniface et al., 2009; Yao et al., 2009). In the studies outlined in this dissertation we have shown that susceptibility and poor outcome of sepsis were associated with higher levels of PGE2 (Figure 4-10), and IFN $\gamma$ (Figure 4-8) and lower levels of IL10 (Figure 4-7). Therefore, we hypothesis that PGE2 can act as a "switch", where in susceptible strains PGE2 can inhibit IL10 production, and increase IFN $\gamma$ levels, thereby promoting Th17 mediated inflammation. Meanwhile, we believe that in resistant strains PGE2 is produced in controlled levels, allowing Th1 and Th17 polarization and subsequent containment of infection. We are even more interested in this interactive role of PGE2 and IL10 as our recent in vitro human studies showed that IL10 mediates protection against severe GAS sepsis (discussed above). In addition, our mouse genome-wide expression pathway analyses identified IL10 pathways to be upregulated in resistant strains at early time points $(8 \mathrm{~h})$ (Figure 4-2) associating high levels of IL10 with resistance to GAS sepsis and better outcomes of infection.

\section{Conclusion}

Our genome-wide transcriptional analyses of infected vs. uninfected resistant and susceptible strains revealed differentially expressed genes that belong to innate immune response pathways that matched our mapped QTLs pathways. Moreover, our analyses of secreted soluble biomarkers (cytokines and prostaglandins) at the studied time points validated the pathways identified through GWAS and GW expression analyses. This shows the power of the systems genetics approach in identifying disease mechanisms, biomarkers and pathways that are associated with differential susceptibility to severe GAS sepsis. Importantly our pathway discovered in mouse matched our findings from clinical studies. This showed that pathways to pathways comparisons between mouse and human can be more readily translated than comparing gene to gene.

\section{Materials and Methods}

\section{Bacteria and Infection Studies Using Mice}

We used hypervirulent M1T1 GAS strain clinical isolate GAS 5448 (Chatellier et al., 2000). Bacterial culture and inoculum used in infection studies were prepared as detailed previously (Aziz et al., 2007). The institutional animal care and use committee (IACUC) at the University of Cincinnati, $\mathrm{OH}$ approved all procedures involving mice and mice tissues described in current study.

\section{Experimental Design of Time Series Infection Studies}

To study the kinetics of cytokines and differential gene expression post GAS sepsis, we designed time course (series) experiments. In these experiments, we infected a 
group of mice belonging to selected highly resistant and highly susceptible ARI-BXD strains and sacrificed the mice at selected time points post infection. In parallel, we injected saline to control group of mice belonging to those selected strains as above. At each time point, we sacrificed the mice, collected blood, using cardiac puncture procedure, and harvested spleens. Blood was used to isolate plasma for cytokine analyses and reconstituted blood (with nuclease-free saline) and preserved blood in RNALater for blood RNA purification at a later stage. Harvested spleens were immediately homogenized in RLT buffer and frozen at $-80^{\circ} \mathrm{C}$ till further purification of spleen RNA.

\section{Processing of Blood and Spleen Samples and Purification of RNA}

We investigated the global gene expression in spleen and blood of infected vs. control groups of mice at selected time points. We selected highly susceptible strains (BXD97, BXD100, and BXD77) and highly resistant strains (BXD87, BXD48, and BXD73) for our analyses. We performed 2-3 biological replicates (experiments) for each selected highly susceptible and highly resistant strains, $n=3$ mice per strain per time point (Figure 4-1). Data represents 7 different experiments with the use of the same strains overlapping multiple cohorts and comparing expression from same strain used in different experiments. We isolated spleen RNA using RNeasy mini (Qiagen, Valencia, $\mathrm{CA}$ ) and isolated blood RNA using Mouse RiboPure kit (Ambion, Austin, Texas) according to manufacturer's protocol. We pooled RNA samples per strain using RNA samples that passed our quality control testing of integrity and purity as detailed below. Globin mRNA was removed from all blood RNA using GlobinClear kit (Ambion). All RNA samples (blood and spleen) were treated for removal of DNA contamination and RNA was further purified by re-precipitation using ethanol precipitation. All RNA samples were checked for RNA purity and integrity. RNA purity was evaluated using the $260 / 280$ and 260/230 absorbance ratios; RNA samples with 260/280 and 260/230 values of $\geq 1.8$ were chosen. Further, RNA integrity was assessed using $1 \%$ RNA denaturing agarose gels. We required clear sharp bands of $18 \mathrm{~S}$ and $28 \mathrm{~S}$ rRNA for all samples compared to a control RNA sample to ensure intactness of RNA.

\section{Microarray Platform and Design}

We used Illumina mouse GW-6 v2.0 BeadChip; probes cover more than 45,200 transcripts per sample, targeting genes and known alternative splice variants. The probes content is derived from the NCBI Reference Sequence (RefSeq) (Build 36, Release 22), supplemented with probes derived from the Mouse Exonic Evidence Based Oligonucliotide (MEEBO) set, as well as exemplar protein-coding sequences described in the RIKEN FANTOM2 database.

We used RNA samples that passed quality control as detailed above and of concentration $\geq 50 \mathrm{ng} / \mathrm{ul}$ for cRNA synthesis using Illumina TotalPrep RNA amplification kit (Ambion) according to manufacturer protocol. The basic outline of the procedure involves reverse transcription of RNA to synthesize cDNA using oligo (dT) primer, followed by in vitro transcription of purified dsDNA to synthesize amplified biotinylated 
cRNA (aRNA). We evaluated purified labeled cRNA using same methods as mentioned above for RNA samples. cRNA samples of good quality were then used to hybridize to Illumina mouseWG-6 v2.0 according to Illumina standard protocols.

This data set consists of arrays processed in four batches or groups, where groups of 2, 4, 1, and 4 beadchips were scanned at a time. Samples from seven different ARIBXD strains were compared at 5 time points $(0,8,12,16$ and 24 hours post infection) belonging to two different tissues, blood and spleen. A single operator processed all arrays using illumina protocol for hybridization, washing and scanning. All samples in a group were labeled on one day and chips were scanned using BeadArray Reader.

\section{Microarray Data Processing and Analysis}

Pooled RNA samples from blood and spleens $(n=60$ samples $)$ were processed using a total of 10 Illumina mouseWG-6 BeadChips. All chips passed quality control and error checking. This data set was extracted and processed using the Bead Studio 3. We applied $75^{\text {th }}$ percentile normalization to all the samples and the resulting expression values were analyzed for statistical significance of differences between susceptible and resistant strains at different time points in the studied tissues.

\section{Identification of Differentially Expressed Genes and Functional Pathways Analyses}

We performed functional analyses of differentially expressed genes from our genome-wide transcriptome analyses using Ingenuity pathways analysis (IPA) (www.ingenuity.com). Each data set containing gene identifiers was uploaded into the online application, and each gene was overlaid onto a molecular network developed from information contained in the ingenuity pathways database. We chose IPA most significant "Canonical Pathways", which are pathways identified from the Ingenuity Pathways Analysis library of pathways to be most significant to the data set. The Canonical Pathways are displayed as $y$-axis in bar charts; $\mathrm{x}$-axis displays the significance in $(-\log (\mathrm{P}-\mathrm{value}))$ where the higher the number the more likely the association is not by chance. In addition a ratio is calculated for each pathway, which is the number of differentially expressed genes divided by total number of molecules in this pathway. Therefore, taller bars have more genes associated with the Canonical Pathway than shorter bars. The significance of the association between the dataset and the pathways was measured in two ways: (1) the ratio (described above) and (2) by Fisher's exact test with $\mathrm{P}<0.001$.

\section{Cytokine Measurements and Analyses}

We studied the kinetics of plasma cytokine production in selected highly resistant and susceptible strains. Groups of 3-5 mice per strain per time point from selected highly susceptible and highly resistant ARI-BXD strains, which were infected with $2 \pm 1.5 \times 10^{7}$ CFU/mouse of a hypervirulent M1T1 GAS strain. In parallel, a control group of 
uninfected mice were used for collection of plasma for estimating normal levels of cytokines in these selected ARI-BXD strains. We euthanized mice and collected blood using cardiac puncture procedure into heparin-coated tubes. We separated plasma from individual mice, added protease inhibitor cocktail to each plasma sample and stored samples at $-20^{\circ} \mathrm{C}$ till further use. We measured cytokines levels in plasma of representative 2 mice per strain of highly resistant (BXD48, 73 and 87) and highly susceptible strains (BXD100, 77 and 97), with exceptions where we assayed more samples from some strains as indicated in footnote of Figure 4-5. We analyzed data collected for time points 8,12 , and 24 hours post-infection. We used a multiplex cytokine ELISA kit from Millipore (Billerica, MA) to detect 4 cytokines: interleukin 6 (IL6), IL10, tumor necrosis factor alpha (TNF $\alpha)$, and gamma interferon IFN $\gamma$. The analyzed data shown in Figures 4-5 to 4-9 are representive of susceptible and resistant groups, where 2 mice per strain (mentioned above) per time point were analyzed, with the exception of BXD 97 at 8 and 12h we used 4 mice and for BXD77 we used 4 and 6 mice at 12 and 24 $\mathrm{h}$ respectively, and BXD87 at 8,12 and $24 \mathrm{~h} 4$ mice were used and one mouse at 8 hours of BXD48. We collected far more plasna samples that are to be assayed. All other data shown in Figures 4-5 to 4-9 are for 2 mice per strain (3 strains used) per time point (4 time points analyzed).

\section{Prostaglandin Studies}

We selected parental strains for studying the role of prostaglandins in modulating differential susceptibility to infection. Mice (6-8 weeks-old mice of DBA/2J susceptible parent strain and the C57B1/6J resistant parent strain) were purchased from The Jackson Laboratory (Bar Harbor, Maine). To test the effect of prostaglandins on differential responses to GAS sepsis, we studied early systemic prostaglandin $\mathrm{E}_{2}\left(\mathrm{PGE}_{2}\right)$ production (within the first 2 hours of infection) in 30 minute intervals (30, 60, 90 and 120 minutes) following inoculation. We infected mice with $2 \times 10^{7} \mathrm{CFU} /$ mouse of the hypervirulent M1T1 GAS strain, and sacrificed 4 mice per strain per time point. Bacterial suspensions used for infection of mice were prepared as detailed in Aziz et al. (2007). We collected blood using a standard cardiac puncture procedure and dissected the spleens and homogenized them using a tissue homogenizer, TH (Omni International, Marietta, GA) in $2 \mathrm{ml}$ of PBS. Tissue homogenates were stored frozen at $-20^{\circ} \mathrm{C}$ and sent on dry ice to the University of Michigan, Ann Arbor, MI. The homogenates were thawed then centrifuged at high speed (13,000 RPM) for 5 min to remove particulates. PGE $_{2}$ levels were quantified in spleen homogenate supernatants using an enzyme-linked immunosorbent assay (ELISA) $\mathrm{PGE}_{2}$ kit according to the manufacturer's instructions (Assay Designs, Ann Arbor, Michigan).

\section{Statistical Analyses}

We used ANOVA and $t$ unpaired test to assess significance of differentially expressed genes in either resistant or susceptible group at a given stage of infection compared to its uninfected control group, using GeneSpring GX 11.0 (Agilent technologies, Inc.). Normalization of microarray data is detailed above. 
We used Kolmogorov-Smirnov test to assess normal distribution of our cytokine data, we found that our cytokine data was not normally distributed, and since the number of samples in some of the tested groups was less than 10, we didn't apply any

normalization methods. Since our data showed that samples had non-normal distribution, we applied the non-parametric tests detailed below To assess statistical significance of differences in levels of different cytokines across time points analyzed we used KruskalWallis test. We evaluated the statistical significance of levels of a given cytokine at a given time point in resistant vs. susceptible strains by using Mann-Whitney test. In all the statistical tests we performed, a P-value less than 0.05 was considered significant. All data was analyzed using GraphPad Prism (La Jolla, CA). 


\title{
CHAPTER 5. ANALYSIS OF CELLULAR POPULATIONS MODULATING DIFFERENTIAL RESPONSE TO SEVERE GAS SEPSIS
}

\begin{abstract}
Summary
We have previously mapped quantitative trait loci (QTLs) associated with differential susceptibility to severe GAS sepsis using advanced recombinant inbred (ARI) ARI-BXD strains (Abdeltawab et al., 2008). We narrowed down these QTLs and parsed candidate genes into pathways associated with severity to GAS sepsis. We then compared pathways discovered through in vivo genome-wide association studies with those discovered through genome-wide transcriptome analyses and found that they matched quite well and even more interestingly, some of these pathways were interacting with each other. Subsequently, we aimed to explore the role of the key genes in those discovered pathways in modulating differential host responses to GAS sepsis (covered in Chapter 4).

In this chapter, we examined the cellular differences between resistant and susceptible strains to help us further elucidate cellular mechanisms underlying the differentially expressed candidate genes in modulating the differential susceptibility to GAS sepsis. To achieve this, we first aimed to examine and characterize differences in immune cell populations of blood and spleen of selected resistant and susceptible strains under normal physiological conditions and found that there were no significant differences between the susceptible and resistant strains with respect to the numbers and percentages of examined immune cells in both blood and spleen. This suggested that inherent variations in percentages of immune cell population among the immunophenotyped BXD strains were not linked to their differential susceptibility to severe GAS sepsis. Accordingly, we aimed to examine the hypothesis that migratory and functional differences rather than cellular availability might be a possible mechanism of resistance.
\end{abstract}

Subsequently, we aimed to examine the possibility that differences in cellular responses may account for the differential susceptibility to GAS sepsis in our mouse model. Specifically, we wanted to test the hypothesis that effective innate responses in the early stages of infection is directly associated with resolution of GAS infection, and better outcome of GAS sepsis. We based our hypothesis on studies from our group and other groups that showed that neutrophils (Voyich et al., 2004; Buchanan et al., 2006; Nilsson et al., 2006; Soehnlein et al., 2008a; Navarini et al., 2009), macrophages (Goldmann et al., 2004a; Thulin et al., 2006; Goldmann et al., 2009), NK cells (Goldmann et al., 2005b; Takahashi et al., 2007) and dendritic cells (Loof et al., 2008; Cortes and Wessels, 2009; Yong et al., 2009), each play an important role in streptococcal infections according to the site and the stage of infection. We started our cellular dissection by examining the role of neutrophils in differential susceptibility to GAS sepsis. Neutrophils are dominant cells in circulation and thus mediate early innate immune response. It has been shown that recruitment, migratory and functional defects in neutrophils result in increased mortality post bacterial infection (reviewed in [Nauseef, 2007]). 
To examine the likely contribution of neutrophils in modulating differential susceptibility in our GAS sepsis model, we first depleted neutrophils and assessed how this may affect infection outcomes in susceptible vs. resistant ARI-BXD strains. We depleted neutrophils using anti-mouse Ly-6G and Ly-6C (RB6-8C5) (Czuprynski et al., 1994) $24 \mathrm{~h}$ prior to infection and found that this rendered resistant BXD strains susceptible. This was associated with an increased bacterial load in depleted mice vs. control mice. The reversal of phenotype of resistant strains, while susceptibility was exaggerated in susceptible strains, confirmed the key role of neutrophils in controlling GAS sepsis.

We also depleted macrophages using clodronate liposome (Van Rooijen and Sanders, 1994) in susceptible and resistant strains and found similar results with reversal of phenotype of resistant strains and exaggerated susceptibility of the susceptible strains. These results demonstrate the important role of both neutrophils and macrophages in modulating differential host responses in GAS sepsis. We also examined the kinetics of neutrophils and macrophages in the first $24 \mathrm{~h}$ post infection and found no significant differences between susceptible and resistant BXD strains. However, peripheral blood monocytes were significantly higher in susceptible strain at $16 \mathrm{~h}$ post infection further showing that even higher numbers of monocytes did not contribute to better outcome of sepsis.

The above data suggested that the numbers of neutrophils and macrophages are not the determinant of the outcome of infection as susceptible strains had an equal or higher numbers of these cells as the resistant strains. Collectively, our data suggest that functional and/or migratory differences in these cells play an important role in modulating differential susceptibility.

\section{Results and Discussion}

\section{Profiling of Blood Cell Populations across Selected Resistant and Susceptible Strains Reveals Possible Migratory and Functional Differences Rather than Cellular Availability as a Possible Mechanism of Individual Variations in Susceptibility to Severe GAS Sepsis}

Homeostasis is a dynamic system regulated by complex interactions between environmental and genetic factors. Blood cellular percentages, platelet counts and erythroid traits have been associated with several QTLs using both inbred and BXD strains (Chen and Harrison, 2002; Peters et al., 2005; Ferreira et al., 2009) GAS has multiple virulence factors that affect and interfere with host homeostasis, for example, interruption of coagulation system (Tapper and Herwald, 2000; Frick et al., 2007), as one of the causes of pathogenesis.

The parental strains of the BXD panel, C57Bl/6J and DBA/2J, show significant differences in numbers and percentages of lymphocytes and granulocytes populations in blood (Chen and Harrison, 2002; Kile et al., 2003; Petkova et al., 2008). Therefore, we 
wanted to examine if observed differential susceptibility to GAS sepsis was due to differences in availability of immune cells. Hence, we aimed to explore differences in the percentages of immune cells in the blood of selected highly resistant and highly susceptible ARI-BXD strains prior to infection and/or any manipulation. Using complete blood counts (CBC), we profiled five immune cells populations in blood (neutrophils, monocytes, lymphocytes, eosinophils and basophils) of highly susceptible strains (BXD100, BXD90, BXD75) and highly resistant strains (BXD73, BXD87 and BXD69), Figures 5-1 and 5-2.

We found no significant differences between resistant and susceptible strains indicating that differences in response to GAS sepsis is not due to differences in cellular populations of susceptible strains vs. resistant strains. We also found that each strain irrespective of its susceptibility had a unique percentage of different immune cell populations, for example, neutrophil percentages (NE \%) was variable among susceptible strains ranging from $23-37 \%$ while in resistant strains it ranged from $14.7-27 \%$. Showing no significant differences between resistant and susceptible groups, indicating that variability in number of cells is irrespective of their differential susceptibility, and that differential response to GAS sepsis is not related to inherent differences in these immune cells numbers.

\section{Immunophenotyping Analyses of Cellular Population under Normal Physiological Conditions in Spleen of BXD Strains Revealed No Differences in Cellular Numbers or Relative Percentages among Susceptible and Resistant Strains}

We immunophenotyped spleen cellular populations in selected resistant and susceptible BXD strains. The spleen as a blood filtering lymphoid organ, is involved in mounting immune responses to extracellular blood borne pathogens such as GAS (Mebius and Kraal, 2005). We wanted to examine if there were any inherent differences in number of immune cells in spleen due to strains variability. We found no significant differences in the analyzed immune cell populations in spleen among highly susceptible and highly resistant strains under normal physiological conditions (Figure 5-3).

Altogether, these data illustrated how different combinations of polymorphic genes and recombinations, can result in a different manifestation from the ancestral parent of these strains. However, in the current study, variations in numbers of immune cells in both spleen and blood of selected highly resistant and susceptible strains were not linked to differential susceptibility to severe GAS sepsis. Our data suggest that possible migratory and functional differences rather than cellular availability as a possible mechanism of differential susceptibility to severe GAS sepsis.

\section{In vivo Manipulation of Cellular Populations in Susceptible and Resistant Strains: Depletion of Gr-1 ${ }^{+}$Cells and Macrophages Rendered Resistant Strains Susceptible}

To this extent we have established that the numbers of immune cells at physiological state were not different between examined highly resistant and susceptible 
Figure 5-1. Differential blood count of selected susceptible and resistant BXD strains.

We used 4-8 mice per sex, per strain, 8-12 weeks-old. The data show no significant differences between resistant and susceptible strains. Since there were significant differences in NE\% between sexes of same strain (BXD69), we are showing males separate from females for all the results. LY lymphocytes, NE neutrophils, MO monocytes, BA basophiles, EO eosinophils. Significance based on Student t-test $\mathrm{p}<$ 0.01 . 


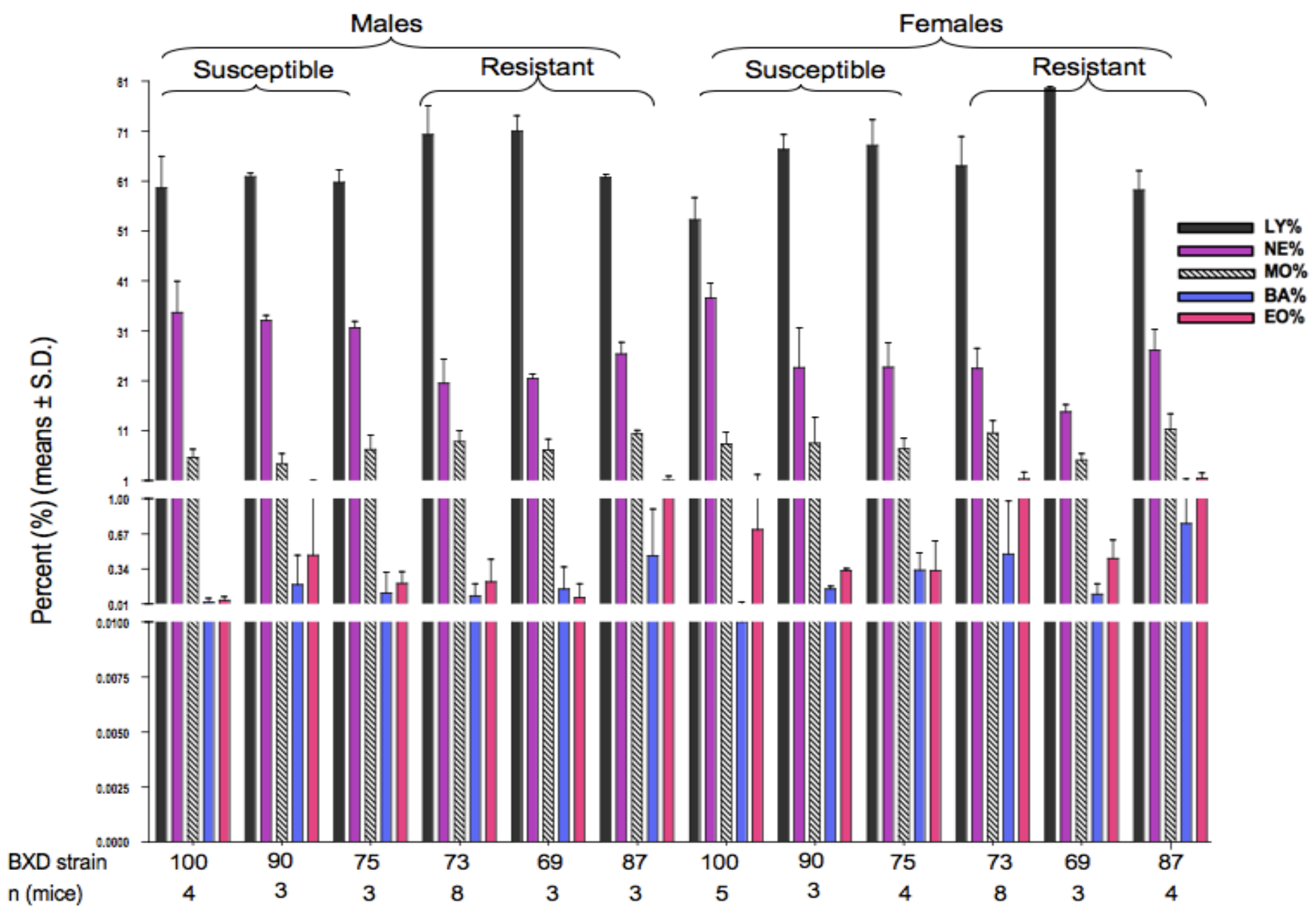


Figure 5-2. Diagram showing a scheme for flow cytometric analysis of immune cell populations in spleen employed in the current study.

We used CD19-FITC to characterize total B cells in spleens (panel A). To characterize main subpopulations of T lymphocytes, we used a mixture of CD3-FITC, CD8-PE and CD4-APC, we defined $\mathrm{CD}^{+}, \mathrm{CD}^{+}$as $\mathrm{T}$ helper cells (panel B), and $\mathrm{CD}^{+}{ }^{+} \mathrm{CD}^{+}$as $\mathrm{T}$ cytotoxic cells (panel C). Natural killer cells were gated as CD3-, CD49b ${ }^{+}$, where CD49b is a pan NK cells marker that is expressed on both parental strains NK cells. We differentiated between granulocytes and macrophages based on granularity (side scatter (SSC)) and CD11b staining as shown in panel E, where we gated on CD11 ${ }^{+}$with high granularity and designated this as total granulocytes (panel G) while CD11 $\mathrm{b}^{+}$cells with lower granularity (SSClo) were assigned a separate gate and designated as macrophages (panel F). Macrophages were then categorized based on their Gr-1 and CD11c expression into resident and inflammatory macrophages (Mø) as shown in panel F. Total granulocytes population (panel G) were then analyzed based on their Gr-1 and SSC into neutrophils and eosinophils as indicated in panel $\mathrm{G}$. 

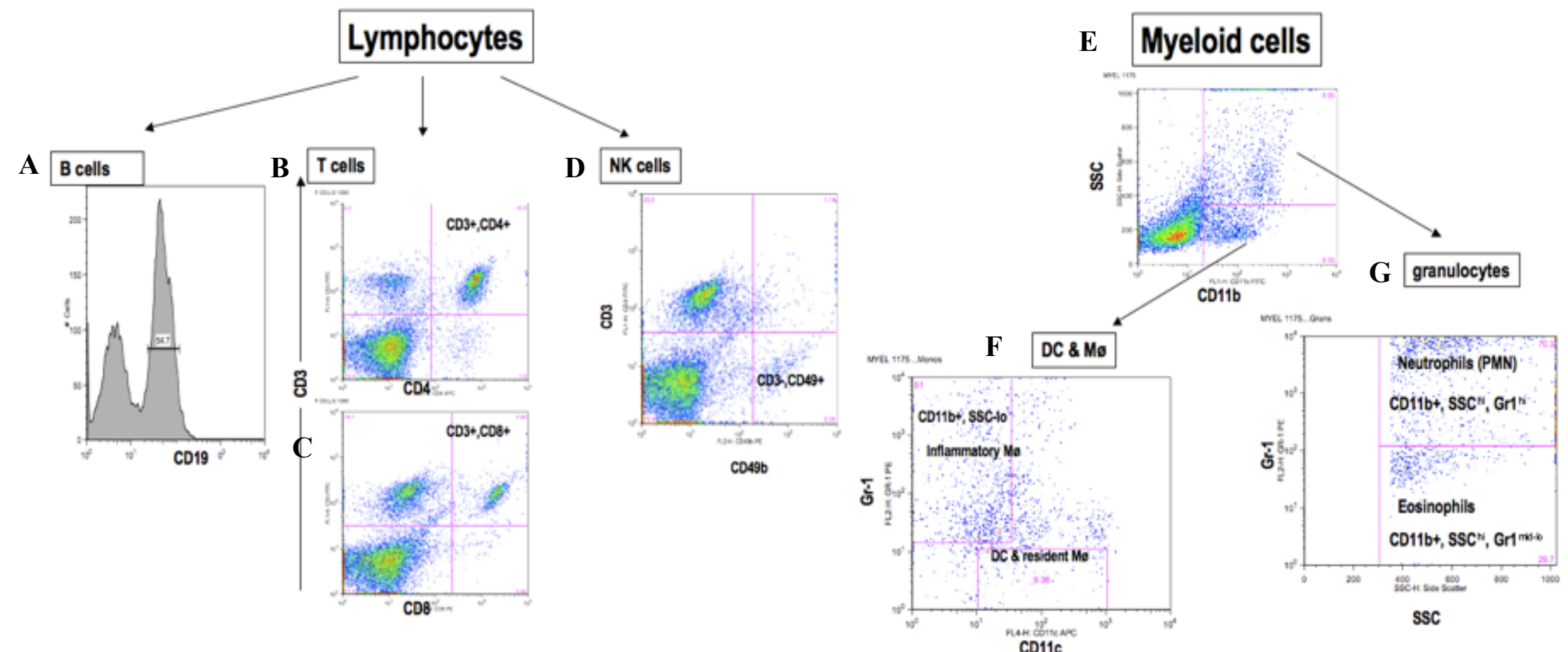


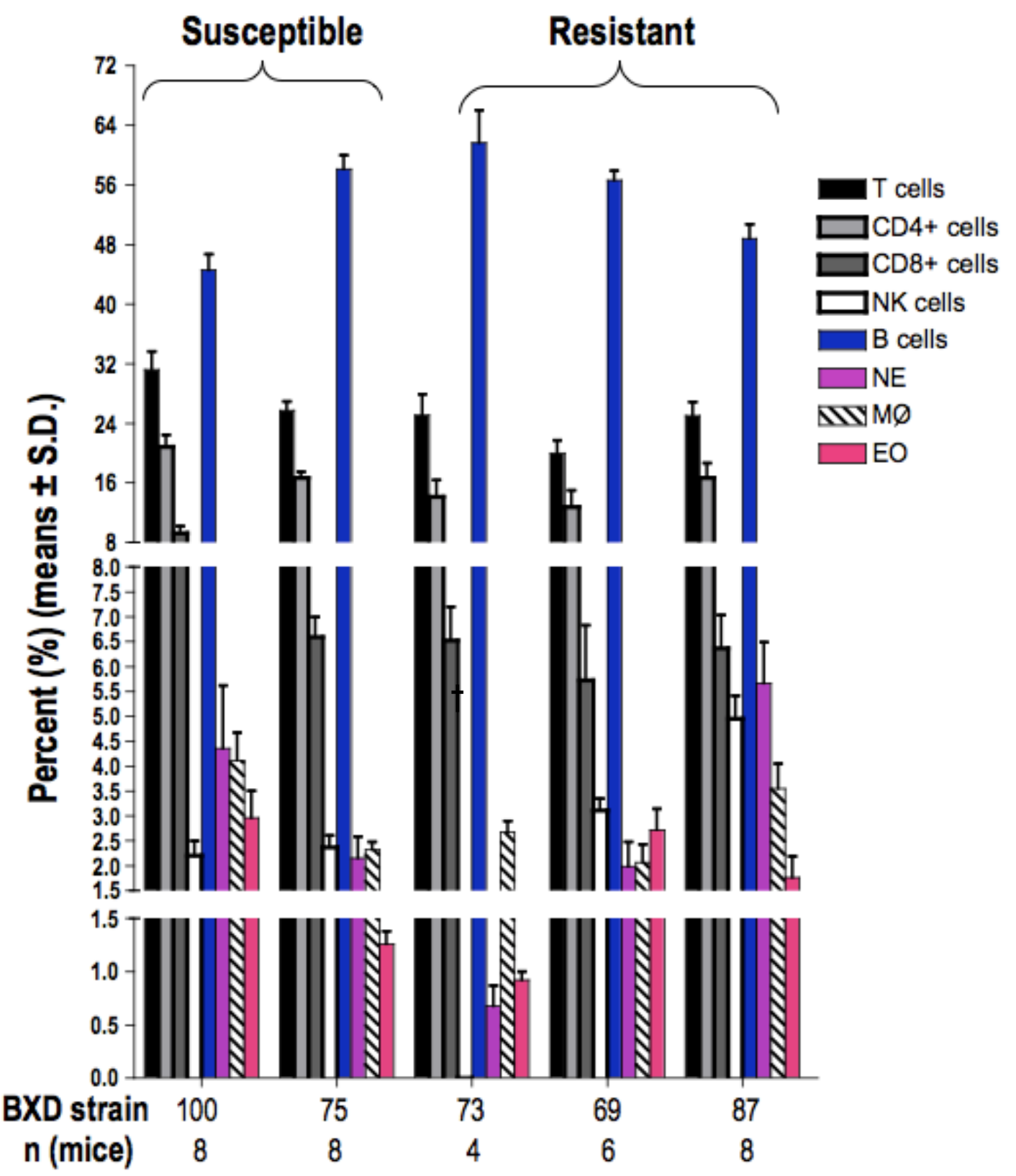

Figure 5-3. Immunophenotyping of spleen cellular populations in selected resistant and susceptible BXD strains.

We used 3-8 mice per sex per strain; age 8-12 weeks old. There were no significant differences in any of the analyzed cellular populations due to sex; therefore, data shown in this Figure represents pooled males and females per strain. There were no significant differences $(\mathrm{P} \leq 0.01)$ between resistant and susceptible strains when comparing different immune cell populations in spleen. NE neutrophils, Mø macrophages, EO eosinophils, and NK natural killer cells. $\uparrow$ NK cells were not analyzed for BXD73. 
strains. Therefore, migratory and/or functional differences rather than cellular availability may possibly account for the differential susceptibility to severe GAS sepsis. Therefore, we aimed to dissect the role of neutrophils and macrophage in differential susceptibility to severe GAS sepsis. We selected neutrophils (PMNs) and macrophages as our genomewide association studies and our transcriptome analyses suggested key roles for these two cell populations in modulating severe GAS sepsis (Abdeltawab et al., 2008).

Neutrophils and macrophages play an important role in modulating host responses to GAS sepsis (Goldmann et al., 2004a; Voyich et al., 2004; Medina and Lengeling, 2005). To dissect the role of these cells, we examined the effect of depleting them in vivo on differential mice susceptibility to severe GAS sepsis. We selected highly susceptible strains (BXD100 and BXD77) and highly resistant strains (BXD48 and BXD69) to test the effect of depleting Gr- $1^{+}$cells on GAS sepsis severity and outcomes (Figure 5-4).

Two days prior to the infection, we quantified the PMNs in all mice, and then at 24 hours prior to the infection, we injected a group of mice i.v. with $200 \mu \mathrm{g}$ of antimouse Ly-6G and Ly-6C (RB6-8C5) antibody (Ab) which depletes Gr1 ${ }^{+}$cells within 1224 hours. This depletion only lasts for $48 \mathrm{~h}$ at which point PMNs are replenished by the bone marrow. In parallel, we injected a matching control group with isotypic control $\mathrm{Ab}$ (purified rat anti-mouse IgG2b $\kappa$ ). To verify neutrophil depletion in the (RB6-8C5) Ab treated group, we measured $\mathrm{CBC} /$ differential blood counts.

We then infected both groups with $10^{7} \mathrm{CFU} /$ mouse of hypervirulent M1T1 GAS strain, and monitored their survival every 2 hours for the first 44 hours then every $8 \mathrm{~h}$ till day 6 post infection, when all surviving mice were sacrificed. We found that the Gr-1 depleted resistant mice were rendered susceptible; indicating the key role of Gr-1 cells (PMNs and monocytes) in host defense against GAS sepsis. We found similar results when we depleted macrophages using clodronate liposomes. Both studies emphasize the role played by neutrophils and macrophages in resistance to severe GAS sepsis. Our results indicated that the first 24 hours of infection are crucial in determining the outcome of infection and the fate of the mice whether the mice will survive or succumb to the infection. This conclusion is based on the finding that despite the fact that PMNs are replenished at 48 hours post depletion and are increased in numbers this did not help anymore in fighting the infection (Figure 5-4). Our results are in concordance with (Goldmann et al., 2004b) where depletion of macrophages prior to or during the first $24 \mathrm{~h}$ of infection affected mice susceptibility and that depletion of macrophages 48 hours post infection had no effect on mice survival.Therefore, our next step was to examine the kinetics of cellular populations in blood in the first 24 hours of infection in highly susceptible and resistant strains.

To examine the kinetics of neutrophils and monocytes in blood post infection, we measured percentages of these cells in susceptible and resistant strains at 8, 12 and $16 \mathrm{~h}$ post infection. We found no significant differences between resistant and susceptible strains (Figure 5-5). We also examined if there were any differences in further earlier time points of infection ( 2 hours) at intervals of 30 minutes, and we again found that there were no significant differences in numbers or percentages of neutrophils between 


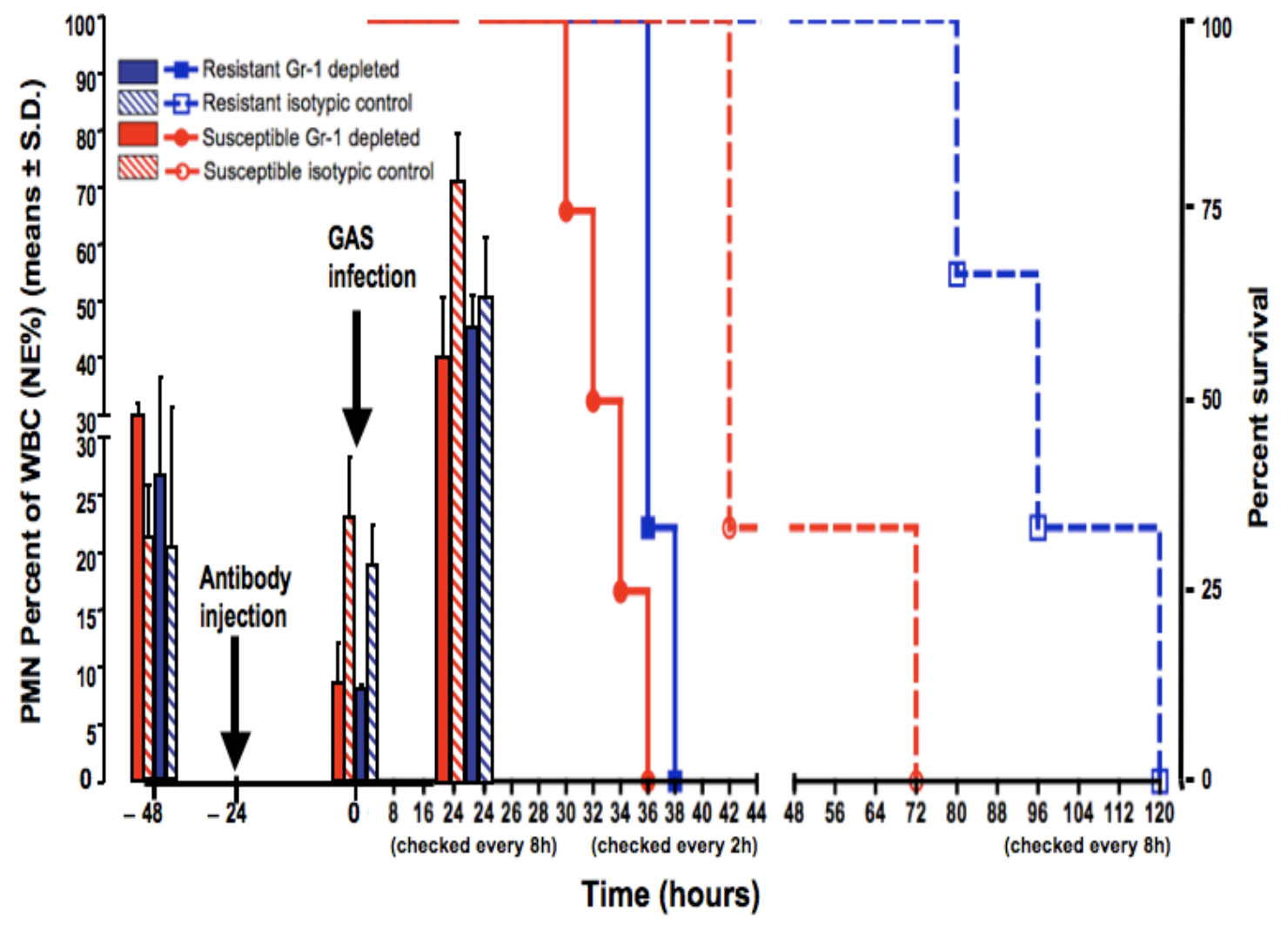

Figure 5-4. Depletion of Gr-1 ${ }^{+}$cells in selected resistant and susceptible BXD strains shows reversal of survival in response to GAS sepsis.

Summary of Gr- $1^{+}$depletion studies showing survival curves as percent survival on the right hand side, where depleted group are in solid lines and control group injected with isotypic control in dashed lines. Resistant strains were rendered susceptible, indicating the beneficiary effect of NEUTROPHILs in modulating host response to severe GAS sepsis. The bar graphs on the left represent percentage of neutrophils pre antibody injection, 24 hours post antibody injection and $24 \mathrm{~h}$ post infection with GAS. 


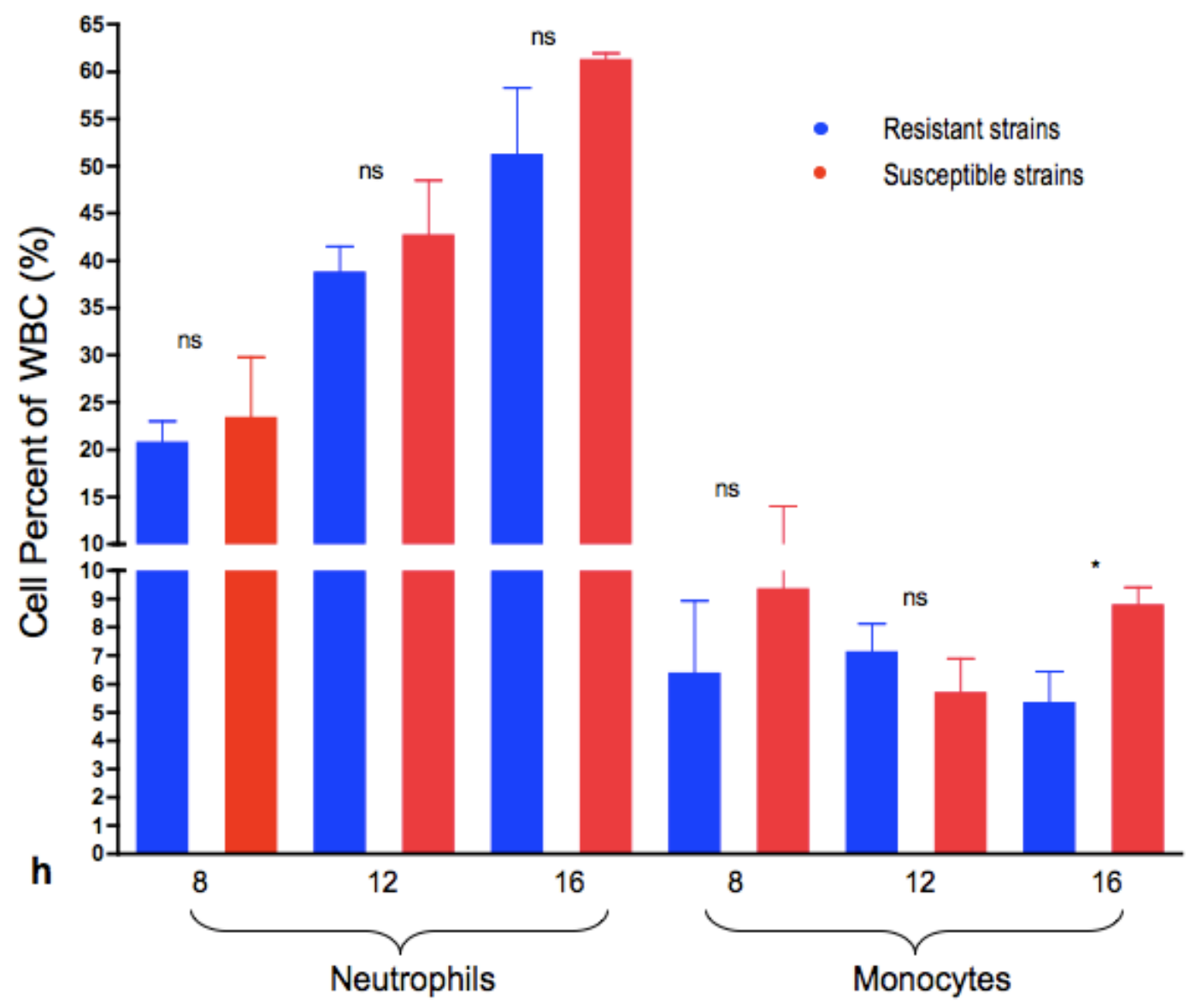

Figure 5-5. Kinetics of neutrophils and monocytes at early time points post GAS infection.

Susceptible and resistant strains were infected with $2 \times 10^{7} \mathrm{CFU} /$ mouse of M1T1 hypervirulent GAS strain. We collected blood from 3-4 mice per strain per time point and evaluated percentages of neutrophils and monocytes at selected time points post infection. There were no significant differences in percentages of neutrophil at examined time points between susceptible strains compared to resistant ones. Interestingly, monocytes were higher at $16 \mathrm{~h}$ post infection in susceptible strains. Significance was calculated using on Student's t-test, with $\mathrm{p} \leq 0.05$ considered significant. 
resistant and susceptible strains at 30-90 minutes post infection. However, at 120 minutes (2h) post infection susceptible strains had significantly higher numbers of neutrophils (Figure 5-6).

\section{Conclusions}

In this chapter, we found that there were no significant quantitative differences in the number of immune cells in blood and spleens of resistant and susceptible strains under normal physiological conditions. This led us to investigate if neutrophils and macrophages play a role in modulating differential response in GAS sepsis. We found that depletion of both cell types prior to, or within $24 \mathrm{~h}$ of GAS infection rendered resistant strains susceptible. We then examined the kinetics of neutrophils and monocytes post infection in susceptible and resistant strains and found no significant differences in numbers post infection. This suggested that numbers of neutrophils and monocytes available may not be a major determinant factors of the outcome of infection as susceptible and resistant strains had an equivalent numbers of these cells. Together our data suggest that functional differences in these cells may play an important role in modulating differential susceptibility.

This led us to initiate studies analyzing the role of neutrophils and pattern recognition receptors, NOD like receptor family (NLR) and in particular Nlrp3 (Craven et al., 2009; Harder et al., 2009; Munoz-Planillo et al., 2009), in modulating differential response to bacterial sepsis. This work along with further in vitro functional assays are being planned and outlined and will be a future direction for the project after completion of the current dissertation.

\section{Materials and Methods}

\section{Experimental Design of Immunophenotyping Studies}

To characterize the main immune cell populations in blood and spleen of BXD strains displaying differential susceptibility to GAS sepsis, we selected highly resistant strains (BXD73, BXD69, and BXD87) and highly susceptible strains (BXD100, BXD90, and BXD75). We collected blood and harvested spleens from 8-16 mice per strain, 8-12 weeks-old, age-matched males and females at physiological conditions. Where the mice were housed in a pathogen-free colony in autoclaved pressurized individual ventilation (PIV) cages (4 mice/cage) at the University of Cincinnati, Cincinnati, OH. The mice used in the immunophenotyping studies were maintained on a 12 hours light dark cycle, and were fed ad libitum sterile irradiated rodent chow (Harlan Teklad, Madison, WI). All procedures involving mice were approved by the institutional animal care and use committee (IACUC) at the University of Cincinnati. 


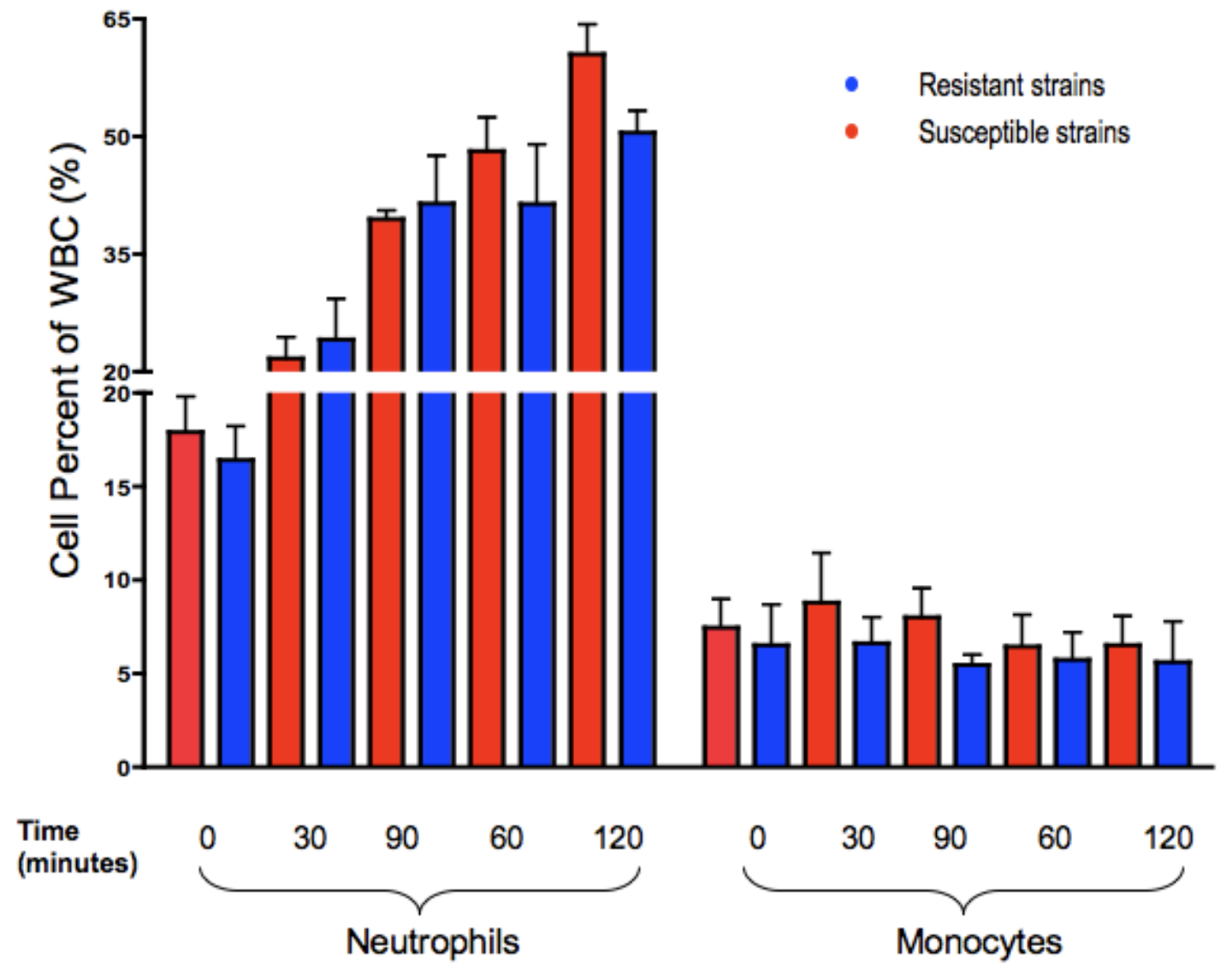

Figure 5-6. Comparison of the kinetics of neutrophils and monocytes numbers in susceptible versus resistant strains during the first 2 hours post GAS infection.

Susceptible and resistant strains were infected with 2 X $10^{7} \mathrm{CFU} / \mathrm{mouse}$ of M1T1 hypervirulent GAS strain. We collected blood from 3-4 mice per strain per time point and evaluated percentages of neutrophils and monocytes at selected time points post infection. There were no significant differences in percentages of neutrophil at examined time points. Significance was calculated using on Student's t-test, with $p \leq 0.05$ considered significant. 


\section{Differential Blood Counts}

We measured numbers and percentage of different immune cells populations in blood using automated complete blood counts (CBC) with differentials. We euthanized age-matched ( $8-12$ weeks-old) mice $(n=4-8 \mathrm{mice} / \mathrm{sex} / \mathrm{strain})$ and collected their blood using cardiac puncture immediately into $0.5 \mathrm{M} \mathrm{K}_{2}$ EDTA coated tubes. We performed $\mathrm{CBC}$ with differentials using Hemavet 950 automated hematology analyzer (Drew Scientific Inc, Oxford CT). We collected data for 5 immune cell populations: lymphocytes, monocytes, eosinophils, basophiles and neutrophils across selected susceptible and resistant ARI-BXD strains.

\section{Flow Cytometric Analyses of Splenocytes}

We used flow cytometric analysis to inspect percentages of different immune cell populations in spleens. We harvested spleens from 8-16 mice/strain, age-matched (8-12 weeks-old) males and females belonging to the above-mentioned BXD strains. We prepared single cell suspension of splenocytes using cell strainer method using a nylon mesh $70 \mu \mathrm{m}$ (BD Biosciences, San Jose, CA). During the following procedures, splenocytes were kept on ice at all possible times. Cell suspensions were pelleted by centrifugation at $400 \mathrm{xg}$ for 5 minutes at room temperature. We discarded the supernatants and lysed RBCs using 1X RBC lysis buffer (eBioscience, Inc, San Diego, CA). We washed the cells twice with FACS buffer (phosphate buffered saline (PBS), 2\% fetal bovine serum (FBS) and $2 \mathrm{mM}$ EDTA), and resuspended the cells in $10 \mathrm{ml}$ of FACS buffer. We counted the splenocytes single cell suspensions using hemocytometer and brought the samples to a concentration of $10^{7}$ cells/ml in FACS buffer.

We stained 106 cells with the following anti-mouse antibodies, CD19-FITC, CD3-FITC, CD8-PE, CD4-APC, CD49b-PE, CD11b-FITC, CD11c-APC, Gr-1-PE (Ly6G and Ly-6C, RB6-8C5), and corresponding isotype controls (BD Biosciences, San Jose, CA). Cell populations and cell surface markers used to identify these populations are listed in Table 5-1. Data acquisition and preliminary analysis were done using BD CellQuest Pro on a BD FACSCalibur flow cytometer (BD Biosciences). Detailed flow cytometeric analyses were done using FlowJo software (Tree Star, Inc, Ashland, OR). We used single color staining for CD19, and staining was analyzed using single histogram statistics (Figure 5-1). We performed 2-color staining for NK cells (CD3 and CD49b), we chose CD49b as an NK cell marker as it is a pan NK cells marker that is expressed on both parental strains (B6 and D2) NK cells. We used 3-color staining for T cells (CD3, CD4 and CD8) and myeloid cells (Gr-1, CD11b and CD11c). Figure 5-1 shows scheme of analysis of different cell populations.

\section{Cellular Depletion Studies}

We used anti-mouse Ly-6G and Ly-6C (RB6-8C5) antibody (Ab) for depletion of $\mathrm{Gr}^{+}$cells. We injected 8 weeks-old males belonging to highly susceptible and highly 
Table 5-1. Cell surface markers that were used to define spleen immune cells populations characterized in the current study.

\begin{tabular}{|c|c|}
\hline Cell Population & $\begin{array}{l}\text { Cell Surface Markers Used to } \\
\text { Define the Cell Population }\end{array}$ \\
\hline T cytotoxic cells (Tc cells) & $\mathrm{CD}^{+}, \mathrm{CD}^{+}$ \\
\hline T helper cells (Th cells) & $\mathrm{CD}^{+}, \mathrm{CD}^{+}$ \\
\hline B lymphocytes (B cells) & $\mathrm{CD}_{19}{ }^{+}$ \\
\hline $\begin{array}{l}\text { Natural killer cells (NK cells) } \\
\text { Neutrophil granulocytes, leukocyte (PMNs) }\end{array}$ & $\begin{array}{l}\mathrm{CD}^{-}, \mathrm{CD}^{-} 4 \mathrm{~b}^{+} \\
\mathrm{CD}^{+} 1 \mathrm{~b}^{+}, \mathrm{SSC}^{\mathrm{hi}}, \mathrm{Gr}-1^{\mathrm{hi}}\end{array}$ \\
\hline Eosinophils (EO) & $\mathrm{CD}_{11} \mathrm{~b}^{+}, \mathrm{SSC}^{\text {hi }}, \mathrm{Gr}-1^{\text {mid-low }}$ \\
\hline Resident macrophage (Mø) \& dendritic cells (DC) & $\mathrm{CD}_{11} \mathrm{~b}^{+}, \mathrm{SSC}^{\mathrm{low}}, \mathrm{Gr}-1^{-}, \mathrm{CD} 11 \mathrm{c}^{+}$ \\
\hline
\end{tabular}

resistant strains with $200 \mathrm{ug} / \mathrm{mouse}$ of RB6-8C5 Ab (iv tail) injections (Czuprynski et al., 1994). In parallel, we injected a control group of mice with $200 \mathrm{ug} / \mathrm{mouse}$ of isotypic control $\mathrm{Ab}$ of purified rat anti-mouse IgG $2 \mathrm{~b} \kappa$. We confirmed depletion by measuring neutrophils in blood 24 hours post Ab injections. We infected control group and $\mathrm{Gr}-1^{+}$ depleted group with $107 \mathrm{CFU} /$ mouse of hypervirulent M1T1 GAS strain. Bacterial suspensions used for infection of mice were prepared as detailed in (Aziz et al., 2007).We monitored mice survival every 2 hours for the first 44 hours until all depleted mice expired and then every $8 \mathrm{~h}$ till day 6 post infection when all surviving mice were sacrificed. Similarly, we depleted monocytes and tissue macrophages using iv tail injection of clodronate-loaded liposomes (CLLs) as previously described in (Van Rooijen and Sanders, 1994). We followed same design for infection as above where control group were injected with PBS-loaded liposomes.

\section{Data Analysis and Statistical Analyses}

We used FlowJo software (Tree Star, Inc, Ashland, OR) for analysis of flow cytometry data. Statistical analysis and graphical presentation of flow cytometry and CBC/differential data was done in GraphPad Prism (GraphPad Software, La Jolla, CA). For granulocyte depletion studies, survival fractions were estimated using Kaplan-Meier method and curves generated were compared using log rank test. In various experiments, statistical significance was based on Student's t-test, a P-value of $\leq 0.01$ was considered significant. 


\section{CHAPTER 6. SUMMARY FUTURE DIRECTIONS AND CONCLUSIONS}

\section{Summary}

In the studies outlined in this dissertation, we aimed to delineate host genetic factors modulating differential susceptibility to severe GAS sepsis.

Our laboratory has previously found an association between variations in host HLA-II alleles and the severity of disease outcomes. These findings were based on extensive epidemiological studies, which were initiated based on two important findings. First, the isolation of genetically identical GAS strains from both severe and non-severe cases of GAS sepsis underscored the role of host variation in modulating outcome of infection (Chatellier et al., 2000). Secondly, subsequent studies showed that there were variations in the magnitude of inflammatory responses elicited by the same GAS superantigens (SAgs) and that this correlated with the severity of GAS sepsis outcomes (Norrby-Teglund et al., 2000). Because SAgs are presented to T cells by HLA-II molecules, they hypothesized that host HLA-II allelic variations could be modulating the observed differential response to the same GAS isolate (Chatellier et al., 2000).

Therefore, extensive epidemiological studies were undertaken to determine the distribution of HLA-II haplotypes among patients with varying degrees of invasive GAS infections. Kotb et al. (2002) found associations between certain HLA-II haplotypes and high risk for STSS and that other haplotypes were associated with protection from STSS and/or NF (Kotb et al., 2002; Kotb et al., 2003). These breakthrough studies were then taken a step further to study mechanisms of interactions of the ternary complex of HLAII-SAgs-TCR in vivo and in vitro. Validation and dissection of the role of in HLA-II variation in GAS sepsis were conducted using HLA-tg mice and bare lymphocyte syndrome (BLS) cell lines expressing either high risk or protective HLA-II alleles (Nooh et al., 2007). The intriguing HLA-II studies in our laboratory are providing us with new exciting mechanistic data each day.

Despite the strong association between severity of GAS sepsis and host HLA-II polymorphism, GAS sepsis is a complex trait disease, affected by multiple interacting mechanisms. This elusive bacterium is rich in many other virulence factors, beside SAgs, of which may interact with additional host factors, other than HLA-II, to modulate disease outcomes. Therefore, we saw a great potential in adopting a systems approach to explore additional host factors modulating the severity of GAS sepsis.

A systems genetics approach is a hypothesis-free approach made feasible due to the coupling of powerful genetic and bioinformatics tools (Morahan and Williams, 2007; Chesler et al., 2008). As detailed in the introductory chapter of this dissertation, ARIBXD strains, which were used as a genetic reference population in our studies, are a welldefined, genetically diverse mouse population. These strains are well defined as they are heavily genotyped and their ancestral parents are fully sequenced (Williams et al., 2001; Peirce et al., 2004; Shifman et al., 2006). This coupled with statistical analyses tools offered by the WebQTL platform (Wang et al., 2003; Peirce et al., 2008) provides 
researchers, like myself, a great advantage of allowing us to easily link our measured traits of interest with mice genotypes and map genetic loci associated with observed differential response. The ARI-BXD strains, being genetically diverse, offer a huge advantage of having a vast genetic pool for mapping studies. This is in contrast to common inbred mice whose genetic pool is limited, thus in using ARI-BXD we are trying to mimic the genetic diversity of the human population.

Systems genetics approaches are novel in GAS sepsis field, and have just been recently employed in other infectious diseases (Hardy et al., 2001; Miyairi et al., 2007). To take advantage of such approaches, we first aimed to carefully account for and to take into consideration any non-genetic or environmental variations that might affect mice differential response to GAS sepsis. Our analyses revealed that among the various cofactors, age was the only significant cofactor that confounded differential response to GAS sepsis (Aziz et al., 2007). This was predictable based on known effects of aging on innate immune responses (Gomez et al., 2008) and in particular on those relating to sepsis (Turnbull et al., 2009). Interestingly, a recent study has dissected such age-related differences in immune responses in relation to severe GAS sepsis in mice (Goldmann et al., 2010). Based on our studies, we standardized an age range for our mice to be between 40 to 120 days that reflects differential response and in later studies of dissecting the mechanisms of differential susceptibility we used age range of 60 to 90 days.

We then mapped disease-associated traits of survival, bacteremia, and bacterial dissemination to organs to 2 QTLs on Chr 2. The survival phenotype also mapped to an additional QTL on Chr X (Abdeltawab et al., 2008). Excitingly, we had a strong cisacting QTL and the genes within these QTLs were polymorphic and associated with immune response pathways which made biological sense. We then narrowed down our QTLs using multiple bioinformatics tools including linkage analyses, SNP polymorphism, gene ontology, and pathway analyses. We then validated selected key genes within our candidate pathways by comparing gene expression levels (qPCR) in selected infected vs. uninfected highly resistant and highly susceptible BXD strains.

Collectively our data suggested the involvement of two key pathways of IL1 and prostaglandins in modulating differential susceptibility to GAS sepsis (Abdeltawab et al., 2008). For the survival phenotype, we found that genes within all 3 QTLs (two on Chr 2 and one on Chr X) fell in the same or interactive pathways. In addition the QTL on Chr X also harbors genes likely to be involved in host-mediated pathogenesis (e.g. thrombosis, and coagulation). However, to further embrace our unbiased approach of systems genetics and to dissect mechanisms of differential susceptibility to severe GAS sepsis, we sought to compare genome-wide (GW) differential gene expression associated with differential response at different stages of infection. We designed these experiments so that we can gain insight into the dynamics of the host immune response during the infection. We compared GW differential gene expression of infected vs. uninfected mice of highly resistant and susceptible strains at selected times post infection.

We aimed to explore the basis of how mice react differently based on their genetic differences, which modulated the outcome of infection into the observed differential 
susceptibility. In addition, we aimed to delineate stages of the infection at which divergence in response between susceptible and resistant strains occurs, i.e. stage at which their fates are being decided. We were excited to find that the same or interacting pathways were also pulled out with this GW transcriptome analysis approach (Chapter 4 in this dissertation). Moreover, we found that certain pathways were associated with certain times post infection i.e. infection stage-specific. This was exemplified by changes in the IL10 signaling pathways, which were only seen in resistant strains only at $8 \mathrm{~h}$ post infection (in blood as studied "organ"). This will help us tremendously in understanding the molecular mechanisms underlying each stage of the infection. To add to the excitement, IL10 cytokine levels correlated with protection from disease severity, as IL10 levels were significantly higher in resistant strains $(\mathrm{p}<0.01)$ at early times post infection. Interestingly, we found significant differences between susceptible and resistant strains only in levels of IL10 and IFN $\gamma$ and at certain times post infection corresponding with the expected time and role of each cytokine in sepsis modulation. The levels of IL10 were significantly higher in resistant strains starting at $8 \mathrm{~h}$ but showed no differences at $24 \mathrm{~h}$. This suggests that IL10 may play an active role in regulating immune response within the early stage of the infection.

This becomes exceedingly exciting, when taken together with our in vitro HLA-II studies which showed that the protective HLA-II alleles induce high levels of IL10 production, suggesting an active suppression as mechanism of protection against STSS. Further support was achieved via in vitro studies, where adding exogenous r-IL10 to PBMCs isolated from individuals expressing high-risk HLA alleles decreased their SAginduced proliferative and cytokine responses. Meanwhile, adding anti-IL10 to PBMCs isolated from individuals expressing protective HLA alleles increased their SAg-induced responses (Nooh and Kotb in press). The IL10 story gets even far more exciting with our microarray data on tissue samples from patients with severe and non severe GAS sepsis, which showed the IL10 signaling pathways as top differentially expressed pathways associated with disease outcomes (Kansal and Kotb in final preparation).

To further add to all the above exciting results, and to shape a bigger picture, our prostaglandin studies revealed that high levels of PGE2 were associated with increased severity of sepsis in inverse correlation with IL10 levels (chapter 4 in this dissertation). This is in agreement with previous studies showing that PGE2 can down regulate the production of IL10 and drives immune cellular responses towards an exaggerated Th17 inflammatory response (Boniface et al., 2009; Yao et al., 2009). However, it has been shown that PGE2 effects are affected by its concentration, type of cells it is acting upon, status of the relevant receptors activated and the site and stage of infection (Bos et al., 2004; Tober et al., 2007; Sakata et al 2010.). Therefore, it seems that a very fine balance of its concentration is required for an effective immune response. This led us to formulate our hypothesis that PGE2 acts as a suppressor of IL10 production in susceptible strains, where at high concentrations of PGE2, IL10 will be inhibited and Th17 inflammation ensues with severe sepsis as an outcome. To test this hypothesis at a cellular mechanism level, we first wanted to profile cellular population percentages across resistant and susceptible strains under physiological conditions. In doing so, we wanted to account for any quantitative differences in number of immune cells of resistant vs. susceptible strains 
prior to infection that might affect their differential response. We immunophenotyped cells from blood and spleen of selected highly resistant and highly susceptible strains and found no quantitative differences between these stains under normal physiological conditions. Furthermore, there were no significant differences in number of neutrophils post infection at selected times post infection.

Our finding of no significant differences in numbers of immune cells between susceptible and resistant strains was very interesting, as it has been previously noted that both qualitative and quantitative differences in neutrophils affect outcomes of infection (Nauseef, 2007). However, an interesting recent study showed that in severe bacterial infections including GAS infection, high bacterial loads induced depletion of bone marrow neutrophils, as it triggered TLR2 activation leading to over-activation of immune response mechanisms and blocking of recruitment of neutrphils to infectious abscess, as bone marrow became depleted of neutrophils (exhausted bone marrow) (Navarini et al., 2009).

Our data continue to point to the important role played by neutrophils and macrophages in modulating the severity and outcomes of GAS sepsis. GAS has developed specific mechanisms to evade killing by these two cellular populations. For example, studies have shown invasive GAS strains to specifically develop mechanisms to evade PMN killing. For example, GAS produces virulence factors that specifically impair PMN chemotaxis, migration and function. C5a peptidase (ScpA) degrades host C5a while IL8 degrading enzyme (ScpC) degrades host IL8 and thus impairing neutrophil chemotaxis (Sjolinder et al., 2008; Gleich-Theurer et al., 2009). Another example of GAS interfering with PMN killing mechanisms is Sda1, a potent DNase produced by M1T1 (GAS strain under study). Sda1 dissolves neutrophil extracellular traps (NETs) allowing the bacteria to escape the entrapment and killing by PMN (Aziz et al., 2004b; Buchanan et al., 2006; Walker et al., 2007). Studies analyzing the role of macrophages in modulating differential susceptibility to GAS, using transcriptome analyses of GAS infected macrophages; found that GAS stimulates an "unusual" macrophage response. As the transcriptional profiles were a mix of pro and anti-inflammatory response, including profiles characteristic of both M1 and M2 macrophages (Goldmann et al., 2007). Nonetheless, PMN and macrophages are in constant interplay, where in GAS sepsis, PMN release antimicrobial LL37 and heparin binding protein (HBP), thereby increasing the extravasations of inflammatory monocytes (Soehnlein et al., 2008b). Put together with our results, we can hypothesize that functional and/or migratory differences in neutrophils and/or monocytes between susceptible and resistant strains might modulate their differential response to GAS sepsis.

In conclusion, we aimed to determine host immunogenetic factors modulating differential host responses to GAS sepsis. To accomplish this aim, we employed systems genetics approaches to study the genetic and molecular basis of host-pathogen interactions leading to differential responses to GAS severe sepsis. We assessed variations in traits associated with severity of GAS sepsis across a genetically diverse reference population (ARI-BXD strains). We then performed genome-wide association scans (GWAS), mapping differential susceptibility to QTLs on mouse Chr 2 and X. 
Combining genome-wide differential gene expression analyses with GWAS, and further biomarker and cellular validations, indicated that PGE2 pathways and IL10 signaling pathways modulate differential responses to GAS sepsis. In addition, our data suggested that prostaglandin itself might act as a key modulator, controlling a network of genes involved in innate immune response to GAS sepsis. We also showed using transcriptome analyses and soluble biomarker analyses, that pathways involving activation of IL10 signaling and IL10 high levels were associated with resistance to infection. Our contribution to the vertical growth of the GAS sepsis field is that we highlighted interactions of prostaglandins and IL10 with networks of candidate genes affect host differential susceptibility to severe GAS sepsis. Our future studies will be focused on further dissecting the molecular mechanisms of the differential susceptibility, these are outlined below.

\section{Future Directions}

Studies outlined in this dissertation have answered the original questions we asked, and raised other questions for follow up in future studies. I would like to believe that my studies helped in setting the foundation for future studies to answer some of the raised questions. I believe that with the knowledge we gained from my studies and the tools we are currently acquiring, answers to some of the raised questions are very plausible in the near future. However, some questions might remain as open-ended questions, which I hope we will someday answer to help us reach the laboratory mission of moving from bench side to bedside and finding better therapeutics that take into account individual variations and that incorporate the information generated to develop more targeted interventions for this aggressive disease.

\section{Dissection of Migratory and Functional Differences of Immune Cell Populations between Susceptible and Resistant Strains}

Our results strongly suggested that migratory and/or functional differences in neutrophils and/or monocytes between resistant and susceptible strains are governing differential response to GAS sepsis, as there were no significant differences in numbers of immune cells of blood and spleen of differentially susceptible strains. Moreover, comparison of neutrophil counts in susceptible and resistant strains post infection showed no association with differential susceptibility. Studies of various GAS virulence factors showed that several affect neutrophil recruitment, migration, and killing mechanisms (Buchanan et al., 2006; Nilsson et al., 2006; Sjolinder et al., 2008; Sumby et al., 2008; Zinkernagel et al., 2008; Lin et al., 2009; Alexander et al., 2010). Although these studies gave us insight into how the bacteria affect neutrophil killing mechanisms, however, the focus of these studies was to dissect bacterial virulence factors rather than to investigate differences in host neutrophils killing mechanisms that can vary between resistant and susceptible individuals.

I believe that studies to test our hypothesis of differences in migratory and functional properties of PMNs and macrophage as a means of host differential 
susceptibility to GAS sepsis will give us great insight into mechanisms involved in differential susceptibility to severe sepsis. To compare differences in functionality of these cells, we will select highly susceptible and highly resistant BXD strains and isolate neutrophils from blood and macrophages from peritoneum, spleen and bone marrow. Then we will compare differences in phagocytic capacities, and production of reactive oxygen species (ROS) and reactive nitrogen species (RNS). In addition, we will measure effective bacterial killing by neutrophils/macrophages isolated from susceptible vs. resistant mouse strains, as it has been shown that although phagocytosized, GAS can survive intracellularly in APCs including neutrophils and monocytes (Medina et al., 2003a; Medina et al., 2003b; Staali et al., 2006; Thulin et al., 2006).

Based on the mapped QTLs and genes associated with GAS severity as well as our present neutrophil depletion studies, we are tempted to believe that the neutrophils/macrophages of the resistant strains will have a better bactericidal activity compared to the susceptible strains. In case we fail to observe expected differences in their in vitro function, an alternate approach would be to test if there are differences in the migration of these cells from lymphoid organs to periphery or vice versa following infection. We will use several in vivo and in vitro methods to study neutrophil migration.

For studying in vivo migration of neutrophils, we will use mouse air pouch technique. Air pouches will be formed on the back of susceptible and resistant BXD mice by subcutaneous injection of sterile air. Mice will then be infected intravenously with GAS and sacrificed at 1-4h post infection. The cells migrated in the pouches will be collected in sterile PBS, and counted with a hemocytometer. Leukocyte subpopulations will be distinguished by flow cytometry. Other studies have shown that neutrophils accounted for 80-95\% of accumulated cells (Ben Yebdri et al., 2009; Hattori et al., 2010; Heissig et al., 2010). In vitro neutrophil transmigration assay will be carried out in a Boyden chamber system. These studies should give us insight into the cellular mechanisms modulating susceptible and resistant strains differential susceptibility to GAS sepsis.

\section{Analysis of Relationship of Prostaglandins and Modulation of Differential Response to GAS}

PGE2 acts as a fast acting, delicately balanced lipid mediator that can act as either an immunosuppressor or immunoactivator depending on PGE2: a) concentration, b) cells it is activating, c) combination of receptors being activated, d) nature of activation signal and e) stage of infection (Bos et al., 2004; Tober et al., 2007; Sakata et al., 2010). PGE2 as an immunosuppressor promotes inflammation, induces IL6 and causes vasodilatation, and vascular leakage; it can also reduce PMN migration and bacterial killing by macrophages, by blocking production of reactive oxygen intermediates (ROI) (Aronoff et al., 2004; White et al., 2005; Nakayama et al., 2006; Huang et al., 2007; Serezani et al., 2007). However, recent studies have shown that it can be a regulator of that immune inflammation (immunoactivator) by activation of Th1 differentiation and Th17 expansion (Boniface et al., 2009; Yao et al., 2009; Sakata et al., 2010). 
If the fine balance of this mediator is disrupted, it can lead to serious pathological consequences. For example alterations in the amounts of PGE2 are associated with high risk of infection with HIV, cancer, and malnutrition (Lima et al., 2006; Anstead et al., 2009; Greenhough et al., 2009). In promoting Th1 differentiation, PGE2 induces the production of IFN $\gamma$ and via its EP4 receptor; PGE2 inhibits production of IL10 exacerbating Th17 mediated inflammation (Boniface et al., 2009; Yao et al., 2009). Excitingly, the outlined studies in this dissertation showed that susceptible stains had higher levels of PGE2, and IFN $\gamma$ and low levels of IL10 while the reverse was true for resistant strains. Therefore, we hypothesize that PGE2 can act as a "switch", wherein susceptible strains PGE2 inhibits IL10 production, and increase IFN $\gamma$, exacerbating Th17 mediated inflammation. Meanwhile, in resistant strains, PGE2 is produced at controlled levels, allowing Th1 and Th17 polarization and subsequent containment of infection. We are even more interested in this interactive role of PGE2 and IL10 as our studies in the HLA-tg mice have shown that IL10 mediates protection against severe GAS sepsis. In addition, our genome-wide expression pathway analyses identified IL10 pathways being up regulated in resistant strains at early time points (8h) associating high levels of IL10 with resistance to GAS sepsis and better outcomes of infection.

A likely applicable hypothesis is that PGE2 modulates differential susceptibility on more than one level, by inhibiting IL10 production, increasing IFN $\gamma$ levels, promoting Th17 expansion but into exaggerated response and subsequent altering of neutrophils and/or macrophages recruitment and functionality. However, further studies are needed to dissect its role in each of these levels, therefore as a future direction of the work presented herein, follow up studies dissecting the role of PGE2 in relation to IL10 in modulating differential susceptibility would be quite interesting.

For these studies, to further explore the role of PGE2, one approach is to use knockout mice of receptors relevant to PGE2 immune actions. PGE2 has four receptors, prostanoid E receptor (EP) 1 through 4 (Narumiya et al., 1999; Breyer et al., 2001). EP2 and EP4 receptors are heavily involved in inflammatory and immune responses, migration of antigen presenting cells (APC) and in cancer research (Kobayashi and Narumiya, 2002; Greenhough et al., 2009). A large body of research on prostaglandins associates the net action of PGE2 with the balance by which PGE2 activates "receptor pairs". For example, in blood pressure regulation, PGE2 can activate EP2/EP4 (leading to vasodilatation) or EP1/EP3 (leading to vasoconstriction), depending on the balance it can shift to one action or the other (Zhang et al., 2000).

Therefore, it would be interesting to test knockout mice of EP2 and EP4 receptors, which are involved in inflammatory and immune responses. We have two knockout mice already in line for future studies on the role of PGE2, these are microsomal prostaglandin E synthase 1 (mPGES1) and EP2 KO mice, which were made in DBA/1J and B6 backgrounds respectively. In our ongoing collaboration with Dr. Aronoff, of Univ. Michigan, we would like to determine how PGE2 might modulate disease severity. I predict that EP2-KO mice would be resistant (as resistant as B6 or more), but the main focus is to determine the mechanism of resistance, through functional 
assays, including phagocytosis, production of ROI, and bacterial killing (clearing of bacteria) as detailed above.

\section{Cytokine Profiling Associated with Susceptibility to GAS Sepsis}

We have measured levels of selected cytokines in selected resistant and susceptible strains and found that IL10 is a promising candidate modulator of severity of sepsis as it was associated with differential response to GAS sepsis. I believe that a carefully designed study can be initiated using levels of IL10 at $8 \mathrm{~h}$ and/or $12 \mathrm{~h}$, as both time points had the highest correlation with disease severity (Figure 4-4), across BXD strains and map differential responses due to cytokine level differences. We have previously used survival, bacteremia and bacterial load in spleen as traits to map differential severity of GAS sepsis and all these traits mapped to Chr 2 QTLs (Abdeltawab et al., 2008). However, we have found that survival was the only trait that mapped a QTL to Chr X. This suggested that genes within this QTL might be primarily engaged in host-mediated pathogenesis, which we found true when we inspected this QTL closely (data not shown).

Therefore, we are hoping that cytokine data will reveal QTLs associated with modulation of cytokine response in GAS sepsis. We postulate that this might be a totally different QTL, as it is known that complex interacting regulatory mechanisms govern cytokine production in sepsis (reviewed in [Cohen, 2002; Rittirsch et al., 2008]). In addition, IL10 is of particular interest due to results shown in this dissertation, where it is correlated with better outcome of sepsis and is in inverse relation to PGE2 levels, another mediator that can be used for mapping.

The association of GAS sepsis severity with multiple cytokines exerting their effects temporally underscores the complexity of traits modulating severe GAS sepsis and emphasizes the importance of the time of administration of appropriate cytokine intervention to aid in the prevention of shock in severe GAS sepsis.

\section{Analysis of the Role of Nod-like Receptors as a Candidate Pattern Recognition Receptor Associated with Modulation of Differential Response to GAS Sepsis}

It has been recently found that Streptolysin O (SLO) produced by GAS (Harder et al., 2009) and hemolysin produced by Staphylococcus aureus (Craven et al., 2009) activate nucleotide-binding domain (NOD) like receptor (NLR) pyrin domain containing 3 (NLRP3) inflammasome, indicating that GAS activation of the inflammasome was independent of TLR signaling. NLRP3 inflammasome is involved in the regulation of caspase-1, which in turn regulates the processing and secretion of IL1 cytokine family including IL1 $\beta$ and IL18, which are both parts of the pathways modulating susceptibility to GAS sepsis which were found by both our genome-wide mapping and transcriptional studies. NLR family acts as a pattern recognition mediating cytoplasmic recognition of bacterial products (Ting et al., 2008; Ye and Ting, 2008). It will be particularly interesting to study the role of the inflammasome in our current GAS model given the growing connections and pointers that it might be associated with signaling pathways 
modulating observed differential response. We have started collaboration with Dr. Jenny Ting, at UNC, where I will pursue this project as part of the immediate future directions of my studies. In these studies we are going to use knockout mice of Nlrp3 and ASC (Apoptosis-associated speck-like protein containing a CARD, also known as PYCARD), both mediators have been associated with modulation of NLRP3 inflammasome (reviewed [Cassel et al., 2009; Lamkanfi and Kanneganti, 2010]).

\section{Concluding Remarks}

The studies presented in this dissertation have helped shed some light on host mechanisms of differential susceptibility to GAS sepsis. We were the first to find an association between differential susceptibility to GAS sepsis and QTLs on mouse Chr 2 and X.Some studies have found associations between genes or QTLs on Chr 2 and susceptibility to other infectious diseases (Appendix D), however none were for differential susceptibility to GAS sepsis. We validated our mapped QTLs and parsed the genes within those QTLs into pathways. This allowed us to explore plausible mechanisms of differential susceptibility. The data suggested that prostaglandin might act as a key modulator, controlling a network of genes involved in innate immune response to GAS sepsis. In addition, pathways involving activation of IL10 production and signaling (including PGE2) are likely to be associated with resistance to infection. Despite differences between mouse and human immune systems (Mestas and Hughes, 2004), our results match beautifully with results obtained from human patients and its validation using in vivo humanized HLA-tg mice. This demonstrates the strength of the systems genetics approach employed and how comparisons of pathways rather than genes can help us translate our findings from mouse to human. 


\section{LIST OF REFERENCES}

Wellcome Trust Case Control Consortium. (2007) Genome-wide association study of 14,000 cases of seven common diseases and 3,000 shared controls. Nature 447: 661-678.

Abdeltawab, N.F., Aziz, R.K., Kansal, R., Rowe, S.L., Su, Y., Gardner, L., Brannen, C., et al. (2008) An unbiased systems genetics approach to mapping genetic loci modulating susceptibility to severe streptococcal sepsis. PLoS Pathog 4: e1000042.

Aikawa, C., Nozawa, T., Maruyama, F., Tsumoto, K., Hamada, S., and Nakagawa, I. (2010) Reactive oxygen species induced by Streptococcus pyogenes invasion trigger apoptotic cell death in infected epithelial cells. Cell Microbiol 12: 814-830.

Alexander, L.E., Maisey, H.C., Timmer, A.M., Rooijakkers, S.H., Gallo, R.L., von Kockritz-Blickwede, M., and Nizet, V. (2010) M1T1 group A streptococcal pili promote epithelial colonization but diminish systemic virulence through neutrophil extracellular entrapment. J Mol Med 88: 371-381.

Andersen, P.S., Lavoie, P.M., Sekaly, R.P., Churchill, H., Kranz, D.M., Schlievert, P.M., Karjalainen, K., et al. (1999) Role of the T cell receptor alpha chain in stabilizing TCRsuperantigen-MHC class II complexes. Immunity 10: 473-483.

Anstead, G.M., Zhang, Q., and Melby, P.C. (2009) Malnutrition promotes prostaglandin over leukotriene production and dysregulates eicosanoid-cytokine crosstalk in activated resident macrophages. Prostaglandins Leukot Essent Fatty Acids 81: 41-51.

Anthony, L.S., Ghadirian, E., Nestel, F.P., and Kongshavn, P.A. (1989) The requirement for gamma interferon in resistance of mice to experimental tularemia. Microb Pathog 7: 421-428.

Aronoff, D.M., Canetti, C., and Peters-Golden, M. (2004) Prostaglandin E2 inhibits alveolar macrophage phagocytosis through an E-prostanoid 2 receptor-mediated increase in intracellular cyclic AMP. J Immunol 173: 559-565.

Aronoff, D.M., Peres, C.M., Serezani, C.H., Ballinger, M.N., Carstens, J.K., Coleman, N., Moore, B.B., et al. (2007) Synthetic prostacyclin analogs differentially regulate macrophage function via distinct analog-receptor binding specificities. J Immunol 178: 1628-1634.

Aziz, R.K., Pabst, M.J., Jeng, A., Kansal, R., Low, D.E., Nizet, V., and Kotb, M. (2004a) Invasive M1T1 group A Streptococcus undergoes a phase-shift in vivo to prevent proteolytic degradation of multiple virulence factors by SpeB. Mol Microbiol 51: 123134.

Aziz, R.K., Ismail, S.A., Park, H.W., and Kotb, M. (2004b) Post-proteomic identification of a novel phage-encoded streptodornase, Sda1, in invasive M1T1 Streptococcus pyogenes. Mol Microbiol 54: 184-197. 
Aziz, R.K., Edwards, R.A., Taylor, W.W., Low, D.E., McGeer, A., and Kotb, M. (2005) Mosaic prophages with horizontally acquired genes account for the emergence and diversification of the globally disseminated M1T1 clone of Streptococcus pyogenes. $J$ Bacteriol 187: 3311-3318.

Aziz, R.K., Kansal, R., Abdeltawab, N.F., Rowe, S.L., Su, Y., Carrigan, D., Nooh, M.M., et al. (2007) Susceptibility to severe Streptococcal sepsis: use of a large set of isogenic mouse lines to study genetic and environmental factors. Genes Immun 8: 404-415.

Aziz, R.K., and Kotb, M. (2008) Rise and persistence of global M1T1 clone of Streptococcus pyogenes. Emerg Infect Dis 14: 1511-1517.

Bailey, D.W. (1971) Recombinant-inbred strains. An aid to finding identity, linkage, and function of histocompatibility and other genes. Transplantation 11: 325-327.

Basma, H., Norrby-Teglund, A., Guedez, Y., McGeer, A., Low, D.E., El-Ahmedy, O., Schwartz, B., et al. (1999) Risk factors in the pathogenesis of invasive group A streptococcal infections: role of protective humoral immunity. Infect Immun 67: 18711877.

Ben Yebdri, F., Kukulski, F., Tremblay, A., and Sevigny, J. (2009) Concomitant activation of $\mathrm{P} 2 \mathrm{Y}(2)$ and $\mathrm{P} 2 \mathrm{Y}(6)$ receptors on monocytes is required for TLR1/2-induced neutrophil migration by regulating IL-8 secretion. Eur J Immunol 39: 2885-2894.

Benjamin, W.H., Jr., Turnbough, C.L., Jr., Posey, B.S., and Briles, D.E. (1986) Salmonella typhimurium virulence genes necessary to exploit the Itys/s genotype of the mouse. Infect Immun 51: 872-878.

Bernstein-Hanley, I., Balsara, Z.R., Ulmer, W., Coers, J., Starnbach, M.N., and Dietrich, W.F. (2006) Genetic analysis of susceptibility to Chlamydia trachomatis in mouse. Genes Immun 7: 122-129.

Bessen, D.E. (2009) Population biology of the human restricted pathogen, Streptococcus pyogenes. Infect Genet Evol 9: 581-593.

Boniface, K., Bak-Jensen, K.S., Li, Y., Blumenschein, W.M., McGeachy, M.J., McClanahan, T.K., McKenzie, B.S., et al. (2009) Prostaglandin E2 regulates Th17 cell differentiation and function through cyclic AMP and EP2/EP4 receptor signaling. $J$ Exp Med 206: 535-548.

Bos, C.L., Richel, D.J., Ritsema, T., Peppelenbosch, M.P., and Versteeg, H.H. (2004) Prostanoids and prostanoid receptors in signal transduction. Int J Biochem Cell Biol 36: 1187-1205.

Boyartchuk, V., and Dietrich, W. (2002) Genetic dissection of host immune response. Genes Immun 3: 119-122. 
Boyartchuk, V.L., Broman, K.W., Mosher, R.E., D'Orazio, S.E., Starnbach, M.N., and Dietrich, W.F. (2001) Multigenic control of Listeria monocytogenes susceptibility in mice. Nat Genet 27: 259-260.

Bream, J.H., Carrington, M., O'Toole, S., Dean, M., Gerrard, B., Shin, H.D., Kosack, D., et al. (2000) Polymorphisms of the human IFNG gene noncoding regions.

Immunogenetics 51: 50-58.

Breyer, R.M., Bagdassarian, C.K., Myers, S.A., and Breyer, M.D. (2001) Prostanoid receptors: subtypes and signaling. Annu Rev Pharmacol Toxicol 41: 661-690.

Buchanan, J.T., Simpson, A.J., Aziz, R.K., Liu, G.Y., Kristian, S.A., Kotb, M., Feramisco, J., et al. (2006) DNase expression allows the pathogen group A Streptococcus to escape killing in neutrophil extracellular traps. Curr Biol 16: 396-400.

Caron, J., Loredo-Osti, J.C., Laroche, L., Skamene, E., Morgan, K., and Malo, D. (2002) Identification of genetic loci controlling bacterial clearance in experimental Salmonella enteritidis infection: an unexpected role of Nramp1 (Slc11a1) in the persistence of infection in mice. Genes Immun 3: 196-204.

Carvalho, A., Cunha, C., Pasqualotto, A.C., Pitzurra, L., Denning, D.W., and Romani, L. (2009) Genetic variability of innate immunity impacts human susceptibility to fungal diseases. Int $J$ Infect Dis.

Cassatella, M.A., Locati, M., and Mantovani, A. (2009) Never underestimate the power of a neutrophil. Immunity 31: 698-700.

Cassel, S.L., Joly, S., and Sutterwala, F.S. (2009) The NLRP3 inflammasome: a sensor of immune danger signals. Semin Immunol 21: 194-198.

Chai, L.Y., Netea, M.G., Vonk, A.G., and Kullberg, B.J. (2009) Fungal strategies for overcoming host innate immune response. Med Mycol 47: 227-236.

Chatellier, S., Ihendyane, N., Kansal, R.G., Khambaty, F., Basma, H., Norrby-Teglund, A., Low, D.E., et al. (2000) Genetic relatedness and superantigen expression in group A streptococcus serotype M1 isolates from patients with severe and nonsevere invasive diseases. Infect Immun 68: 3523-3534.

Chen, B.C., Chang, Y.S., Kang, J.C., Hsu, M.J., Sheu, J.R., Chen, T.L., Teng, C.M., et al. (2004) Peptidoglycan induces nuclear factor-kappaB activation and cyclooxygenase-2 expression via Ras, Raf-1, and ERK in RAW 264.7 macrophages. J Biol Chem 279: 20889-20897.

Chen, J., and Harrison, D.E. (2002) Quantitative trait loci regulating relative lymphocyte proportions in mouse peripheral blood. Blood 99: 561-566. 
Chesler, E.J., Lu, L., Shou, S., Qu, Y., Gu, J., Wang, J., Hsu, H.C., et al. (2005) Complex trait analysis of gene expression uncovers polygenic and pleiotropic networks that modulate nervous system function. Nat Genet 37: 233-242.

Chesler, E.J., Miller, D.R., Branstetter, L.R., Galloway, L.D., Jackson, B.L., Philip, V.M., Voy, B.H., et al. (2008) The Collaborative Cross at Oak Ridge National Laboratory: developing a powerful resource for systems genetics. Mamm Genome 19: 382-389.

Churchill, G.A., and Doerge, R.W. (1994) Empirical threshold values for quantitative trait mapping. Genetics 138: 963-971.

Churchill, G.A., and Airey, D.C., and Allayee, H., and Angel, J.M., and Attie, A.D., and Beatty, J., and Beavis, W.D., et al. (2004) The Collaborative Cross, a community resource for the genetic analysis of complex traits. Nat Genet 36: 1133-1137.

Churchill, G.A. (2007) Recombinant inbred strain panels: a tool for systems genetics. Physiol Genomics 31: 174-175.

Cohen, J. (2002) The immunopathogenesis of sepsis. Nature 420: 885-891.

Cole, J.N., McArthur, J.D., McKay, F.C., Sanderson-Smith, M.L., Cork, A.J., Ranson, M., Rohde, M., et al. (2006) Trigger for group A streptococcal M1T1 invasive disease. FASEB J. 20: 1745-1747.

Cortes, G., and Wessels, M.R. (2009) Inhibition of dendritic cell maturation by group A Streptococcus. J Infect Dis 200: 1152-1161.

Craven, R.R., Gao, X., Allen, I.C., Gris, D., Bubeck Wardenburg, J., McElvania-Tekippe, E., Ting, J.P., et al. (2009) Staphylococcus aureus alpha-hemolysin activates the NLRP3inflammasome in human and mouse monocytic cells. PLoS One 4: e7446.

Cunningham, M.W. (2000) Pathogenesis of group A streptococcal infections. Clin Microbiol Rev 13: 470-511.

Cywes Bentley, C., Hakansson, A., Christianson, J., and Wessels, M.R. (2005)

Extracellular group A Streptococcus induces keratinocyte apoptosis by dysregulating calcium signalling. Cell Microbiol 7: 945-955.

Czuprynski, C.J., Brown, J.F., Maroushek, N., Wagner, R.D., and Steinberg, H. (1994) Administration of anti-granulocyte $\mathrm{mAb}$ RB6-8C5 impairs the resistance of mice to Listeria monocytogenes infection. J Immunol 152: 1836-1846.

Davies, H.D., McGeer, A., Schwartz, B., Green, K., Cann, D., Simor, A.E., and Low, D.E. (1996) Invasive group A streptococcal infections in Ontario, Canada. Ontario Group A Streptococcal Study Group. N Engl J Med 335: 547-554. 
Deghmane, A.E., Veckerle, C., Giorgini, D., Hong, E., Ruckly, C., and Taha, M.K. (2009) Differential modulation of TNF-alpha-induced apoptosis by Neisseria meningitidis. PLoS Pathog 5: e1000405.

Elgueta, R., Benson, M.J., de Vries, V.C., Wasiuk, A., Guo, Y., and Noelle, R.J. (2009) Molecular mechanism and function of CD40/CD40L engagement in the immune system. Immunol Rev 229: 152-172.

Ferreira, M.A., Hottenga, J.J., Warrington, N.M., Medland, S.E., Willemsen, G., Lawrence, R.W., Gordon, S., et al. (2009) Sequence variants in three loci influence monocyte counts and erythrocyte volume. Am J Hum Genet 85: 745-749.

Fortin, A., Abel, L., Casanova, J.L., and Gros, P. (2007) Host genetics of mycobacterial diseases in mice and men: forward genetic studies of BCG-osis and tuberculosis. Annu Rev Genomics Hum Genet 8: 163-192.

Frank, S.A. (2002) Immunology and Evolution of Infectious Disease: Princeton (NJ) Princeton University Press.

Frazer, K.A., and Ballinger, D.G., and Cox, D.R., and Hinds, D.A., and Stuve, L.L., and Gibbs, R.A., and Belmont, J.W., et al. (2007) A second generation human haplotype map of over 3.1 million SNPs. Nature 449: 851-861.

Frick, I.M., Bjorck, L., and Herwald, H. (2007) The dual role of the contact system in bacterial infectious disease. Thromb Haemost 98: 497-502.

Frodsham, A.J., and Hill, A.V. (2004) Genetics of infectious diseases. Hum Mol Genet 13 Spec No 2: R187-194.

Garcia-Peydro, M., de Yebenes, V.G., and Toribio, M.L. (2006) Notch1 and IL-7 receptor interplay maintains proliferation of human thymic progenitors while suppressing non-T cell fates. J Immunol 177: 3711-3720.

Gleich-Theurer, U., Aymanns, S., Haas, G., Mauerer, S., Vogt, J., and Spellerberg, B. (2009) Human serum induces streptococcal c5a peptidase expression. Infect Immun 77: 3817-3825.

Goldmann, O., Chhatwal, G.S., and Medina, E. (2003) Immune mechanisms underlying host susceptibility to infection with group A streptococci. J Infect Dis 187: 854-861.

Goldmann, O., Rohde, M., Chhatwal, G.S., and Medina, E. (2004a) Role of macrophages in host resistance to group A streptococci. Infect Immun 72: 2956-2963.

Goldmann, O., Chhatwal, G.S., and Medina, E. (2004b) Role of host genetic factors in susceptibility to group A streptococcal infections. Indian J Med Res 119 Suppl: 141-143. 
Goldmann, O., Lengeling, A., Bose, J., Bloecker, H., Geffers, R., Chhatwal, G.S., and Medina, E. (2005a) The role of the MHC on resistance to group a streptococci in mice. $J$ Immunol 175: 3862-3872.

Goldmann, O., Chhatwal, G.S., and Medina, E. (2005b) Contribution of natural killer cells to the pathogenesis of septic shock induced by Streptococcus pyogenes in mice. $J$ Infect Dis 191: 1280-1286.

Goldmann, O., von Kockritz-Blickwede, M., Holtje, C., Chhatwal, G.S., Geffers, R., and Medina, E. (2007) Transcriptome analysis of murine macrophages in response to infection with Streptococcus pyogenes reveals an unusual activation program. Infect Immun 75: 4148-4157.

Goldmann, O., Sastalla, I., Wos-Oxley, M., Rohde, M., and Medina, E. (2009) Streptococcus pyogenes induces oncosis in macrophages through the activation of an inflammatory programmed cell death pathway. Cell Microbiol 11: 138-155.

Goldmann, O., Lehne, S., and Medina, E. (2010) Age-related susceptibility to Streptococcus pyogenes infection in mice: underlying immune dysfunction and strategy to enhance immunity. J Pathol 220: 521-529.

Gomez, C.R., Nomellini, V., Faunce, D.E., and Kovacs, E.J. (2008) Innate immunity and aging. Exp Gerontol 43: 718-728.

Greenhough, A., Smartt, H.J., Moore, A.E., Roberts, H.R., Williams, A.C., Paraskeva, C., and Kaidi, A. (2009) The COX-2/PGE2 pathway: key roles in the hallmarks of cancer and adaptation to the tumour microenvironment. Carcinogenesis 30: 377-386.

Grizzle, W.E., Mountz, J.D., Yang, P.A., Xu, X., Sun, S., Van Zant, G.E., Williams, R.W., et al. (2002) BXD recombinant inbred mice represent a novel T cell-mediated immune response tumor model. Int J Cancer 101: 270-279.

Guedez, Y., Kotby, A., El-Demellawy, M., Galal, A., Thomson, G., Zaher, S., Kassem, S., et al. (1999) HLA class II associations with rheumatic heart disease are more evident and consistent among clinically homogeneous patients. Circulation 99: 2784-2790.

Harder, J., Franchi, L., Munoz-Planillo, R., Park, J.H., Reimer, T., and Nunez, G. (2009) Activation of the Nlrp3 inflammasome by Streptococcus pyogenes requires streptolysin $\mathrm{O}$ and NF-kappa B activation but proceeds independently of TLR signaling and P2X7 receptor. J Immunol 183: 5823-5829.

Hardy, C.L., Lu, L., Nguyen, P., Woodland, D.L., Williams, R.W., and Blackman, M.A. (2001) Identification of quantitative trait loci controlling activation of TRBV4 CD8+ T cells during murine gamma-herpesvirus-induced infectious mononucleosis.

Immunogenetics 53: 395-400.

Harris, S.G., Padilla, J., Koumas, L., Ray, D., and Phipps, R.P. (2002) Prostaglandins as modulators of immunity. Trends Immunol 23: 144-150. 
Hattori, H., Subramanian, K.K., Sakai, J., Jia, Y., Li, Y., Porter, T.F., Loison, F., et al. (2010) Small-molecule screen identifies reactive oxygen species as key regulators of neutrophil chemotaxis. Proc Natl Acad Sci U S A 107: 3546-3551.

Hauser, A.R., Stevens, D.L., Kaplan, E.L., and Schlievert, P.M. (1991) Molecular analysis of pyrogenic exotoxins from Streptoccus pyogenes isolated associated with toxic shock-like syndrome. J. Clin. Microbiol. 29: 1562.

Heissig, B., Nishida, C., Tashiro, Y., Sato, Y., Ishihara, M., Ohki, M., Gritli, I., et al. (2010) Role of neutrophil-derived matrix metalloproteinase-9 in tissue regeneration. Histol Histopathol 25: 765-770.

Hidalgo-Grass, C., Mishalian, I., Dan-Goor, M., Belotserkovsky, I., Eran, Y., Nizet, V., Peled, A., et al. (2006) A streptococcal protease that degrades CXC chemokines and impairs bacterial clearance from infected tissues. Embo $J$ 25: 4628-4637.

Hoge, C.W., Schwartz, B., Talkington, D.F., Breiman, R.F., MacNeill, E.M., and Englender, S.J. (1993) The changing epidemiology of invasive group A streptococcal infections and the emergence of streptococcal toxic shock-like syndrome. A retrospective population-based study. JAMA 269: 384-389.

Hormaeche, C.E. (1979a) Natural resistance to Salmonella typhimurium in different inbred mouse strains. Immunology 37: 311-318.

Hormaeche, C.E. (1979b) Genetics of natural resistance to salmonellae in mice. Immunology 37: 319-327.

Hornef, M.W., Wick, M.J., Rhen, M., and Normark, S. (2002) Bacterial strategies for overcoming host innate and adaptive immune responses. Nat Immunol 3: 1033-1040.

Huang, S., Wettlaufer, S.H., Hogaboam, C., Aronoff, D.M., and Peters-Golden, M. (2007) Prostaglandin E(2) inhibits collagen expression and proliferation in patientderived normal lung fibroblasts via $\mathrm{E}$ prostanoid 2 receptor and cAMP signaling. Am $J$ Physiol Lung Cell Mol Physiol 292: L405-413.

Ikebe, T., Hirasawa, K., Suzuki, R., Ohya, H., Isobe, J., Tanaka, D., Katsukawa, C., et al. (2007) Distribution of emm genotypes among group A streptococcus isolates from patients with severe invasive streptococcal infections in Japan, 2001-2005. Epidemiol Infect 135: 1227-1229.

Jackson, I.J. (2001) Mouse genomics: making sense of the sequence. Curr Biol 11: R311314.

Jobin, M.C., Gottschalk, M., and Grenier, D. (2006) Upregulation of prostaglandin E2 and matrix metalloproteinase 9 production by human macrophage-like cells: synergistic effect of capsular material and cell wall from Streptococcus suis. Microb Pathog 40: 2934. 
Johansson, L., Thulin, P., Sendi, P., Hertzen, E., Linder, A., Akesson, P., Low, D.E., et al. (2008) Cathelicidin LL-37 in severe Streptococcus pyogenes soft tissue infections in humans. Infect Immun 76: 3399-3404.

Johnson, D.R., Stevens, D.L., and Kaplan, E.L. (1992) Epidemiologic analysis of group A streptococcal serotypes associated with severe systemic infections, rheumatic fever, or uncomplicated pharyngitis. J Infect Dis 166: 374-382.

Johnson, D.R., Wotton, J.T., Shet, A., and Kaplan, E.L. (2002) A comparison of group A streptococci from invasive and uncomplicated infections: are virulent clones responsible for serious streptococcal infections? J Infect Dis 185: 1586-1595.

Joo, M., Kwon, M., Sadikot, R.T., Kingsley, P.J., Marnett, L.J., Blackwell, T.S., Peebles, R.S., Jr., et al. (2007) Induction and function of lipocalin prostaglandin D synthase in host immunity. J Immunol 179: 2565-2575.

Kansal, R.G., McGeer, A., Low, D.E., Norrby-Teglund, A., and Kotb, M. (2000) Inverse relation between disease severity and expression of the streptococcal cysteine protease, $\mathrm{SpeB}$, among clonal M1T1 isolates recovered from invasive group A streptococcal infection cases. Infect Immun 68: 6362-6369.

Kaul, R., McGeer, A., Low, D.E., Green, K., and Schwartz, B. (1997) Population-based surveillance for group A streptococcal necrotizing fasciitis: Clinical features, prognostic indicators, and microbiologic analysis of seventy-seven cases. Ontario Group A Streptococcal Study. Am J Med 103: 18-24.

Kazmi, S.U., Kansal, R., Aziz, R.K., Hooshdaran, M., Norrby-Teglund, A., Low, D.E., Halim, A.B., et al. (2001) Reciprocal, temporal expression of SpeA and SpeB by invasive M1T1 group a streptococcal isolates in vivo. Infect Immun 69: 4988-4995.

Kiang, J.G., and Tsokos, G.C. (1998) Heat shock protein 70 kDa: molecular biology, biochemistry, and physiology. Pharmacol Ther 80: 183-201.

Kile, B.T., Mason-Garrison, C.L., and Justice, M.J. (2003) Sex and strain-related differences in the peripheral blood cell values of inbred mouse strains. Mamm Genome 14: $81-85$.

Kim, H.P., Morse, D., and Choi, A.M. (2006) Heat-shock proteins: new keys to the development of cytoprotective therapies. Expert Opin Ther Targets 10: 759-769.

Klenk, M., Koczan, D., Guthke, R., Nakata, M., Thiesen, H.J., Podbielski, A., and Kreikemeyer, B. (2005) Global epithelial cell transcriptional responses reveal Streptococcus pyogenes Fas regulator activity association with bacterial aggressiveness. Cell Microbiol 7: 1237-1250. 
Ko, D.C., Shukla, K.P., Fong, C., Wasnick, M., Brittnacher, M.J., Wurfel, M.M., Holden, T.D., et al. (2009) A genome-wide in vitro bacterial-infection screen reveals human variation in the host response associated with inflammatory disease. Am J Hum Genet 85: 214-227.

Kobayashi, S.D., Braughton, K.R., Whitney, A.R., Voyich, J.M., Schwan, T.G., Musser, J.M., and DeLeo, F.R. (2003) Bacterial pathogens modulate an apoptosis differentiation program in human neutrophils. Proc Natl Acad Sci U S A 100: 10948-10953.

Kobayashi, T., and Narumiya, S. (2002) Function of prostanoid receptors: studies on knockout mice. Prostaglandins Other Lipid Mediat 68-69: 557-573.

Kotb, M. (1992) Role of superantigens in the pathogenesis of infectious diseases and their sequelae. Curr. Opin. Infect. Dis 5: 364.

Kotb, M. (1995) Bacterial pyrogenic exotoxins as superantigens. Clin Microbiol Rev 8: 411-426.

Kotb, M., Norrby-Teglund, A., McGeer, A., El-Sherbini, H., Dorak, M.T., Khurshid, A., Green, K., et al. (2002) An immunogenetic and molecular basis for differences in outcomes of invasive group A streptococcal infections. Nat Med 8: 1398-1404.

Kotb, M., Norrby-Teglund, A., McGeer, A., Green, K., and Low, D.E. (2003) Association of human leukocyte antigen with outcomes of infectious diseases: the streptococcal experience. Scand J Infect Dis 35: 665-669.

Kotb, M. (2004) Genetics of susceptibility to infectious diseases. ASM News 70 (10): 457-463.

Kustanova, G.A., Murashev, A.N., Guzhova, I.V., Margulis, B.A., Prokhorenko, I.R., Grachev, S.V., and Evgen'ev, M.B. (2006) Protective effect of exogenous 70-kDa heat shock protein during endotoxic shock (sepsis). Dokl Biol Sci 411: 504-507.

Lamkanfi, M., and Kanneganti, T.D. (2010) Nlrp3: an immune sensor of cellular stress and infection. Int J Biochem Cell Biol 42: 792-795.

Lappin, E., and Ferguson, A.J. (2009) Gram-positive toxic shock syndromes. Lancet Infect Dis 9: 281-290.

Leder, L., Llera, A., Lavoie, P.M., Lebedeva, M.I., Li, H., Sekaly, R.P., Bohach, G.A., et al. (1998) A mutational analysis of the binding of staphylococcal enterotoxins B and C3 to the T cell receptor beta chain and major histocompatibility complex class II. $J$ Exp Med 187: 823-833.

Lima, R.G., Moreira, L., Paes-Leme, J., Barreto-de-Souza, V., Castro-Faria-Neto, H.C., Bozza, P.T., and Bou-Habib, D.C. (2006) Interaction of macrophages with apoptotic cells enhances HIV Type 1 replication through PGE2, PAF, and vitronectin receptor. AIDS Res Hum Retroviruses 22: 763-769. 
Lin, A., Loughman, J.A., Zinselmeyer, B.H., Miller, M.J., and Caparon, M.G. (2009) Streptolysin S inhibits neutrophil recruitment during the early stages of Streptococcus pyogenes infection. Infect Immun 77: 5190-5201.

Loof, T.G., Goldmann, O., and Medina, E. (2008) Immune recognition of Streptococcus pyogenes by dendritic cells. Infect Immun 76: 2785-2792.

Low, D.E., Schwartz, B., and McGeer, A. (1997) The reemergence of severe group A streptococcal disease: an evolutionary perspetive. Washington, D.C: American Society for Microbiology Press.

Lynch, M., and Walsh, B. (1998) Genetics and Analysis of Quantitative Traits. Sinauer Associates, Sunderland, Massachusetts.

Mahler, M., Janke, C., Wagner, S., and Hedrich, H.J. (2002) Differential susceptibility of inbred mouse strains to Helicobacter pylori infection. Scand J Gastroenterol 37: 267-278.

Maloney, C.G., Thompson, S.D., Hill, H.R., Bohnsack, J.F., McIntyre, T.M., and Zimmerman, G.A. (2000) Induction of cyclooxygenase-2 by human monocytes exposed to group B streptococci. J Leukoc Biol 67: 615-621.

Marriott, H.M., and Dockrell, D.H. (2006) Streptococcus pneumoniae: the role of apoptosis in host defense and pathogenesis. Int J Biochem Cell Biol 38: 1848-1854.

Mebius, R.E., and Kraal, G. (2005) Structure and function of the spleen. Nat Rev Immunol 5: 606-616.

Medina, E., Goldmann, O., Rohde, M., Lengeling, A., and Chhatwal, G.S. (2001) Genetic control of susceptibility to group A streptococcal infection in mice. $J$ Infect Dis 184: 846852 .

Medina, E., Goldmann, O., Toppel, A.W., and Chhatwal, G.S. (2003a) Survival of Streptococcus pyogenes within host phagocytic cells: a pathogenic mechanism for persistence and systemic invasion. J Infect Dis 187: 597-603.

Medina, E., Rohde, M., and Chhatwal, G.S. (2003b) Intracellular survival of Streptococcus pyogenes in polymorphonuclear cells results in increased bacterial virulence. Infect Immun 71: 5376-5380.

Medina, E., and Lengeling, A. (2005) Genetic regulation of host responses to group A streptococcus in mice. Brief Funct Genomic Proteomic 4: 248-257.

Melvold, R.W., Jokinen, D.M., Miller, S.D., Dal Canto, M.C., and Lipton, H.L. (1990) Identification of a locus on mouse chromosome 3 involved in differential susceptibility to Theiler's murine encephalomyelitis virus-induced demyelinating disease. J Virol 64: 686690. 
Mestas, J., and Hughes, C.C. (2004) Of mice and not men: differences between mouse and human immunology. J Immunol 172: 2731-2738.

Miethke, T., Wahl, C., Heeg, K., Echtenacher, B., Krammer, P.H., and Wagner, H. (1992) T cell-mediated lethal shock triggered in mice by the superantigen staphylococcal enterotoxin B: critical role of tumor necrosis factor. $J$ Exp Med 175: 91-98.

Minami, M., Ohmori, D., Tatsuno, I., Isaka, M., Kawamura, Y., Ohta, M., and Hasegawa, T. (2009) The streptococcal inhibitor of complement (SIC) protects Streptococcus pyogenes from bacteriocin-like inhibitory substance (BLIS) from Streptococcus salivarius. FEMS Microbiol Lett 298: 67-73.

Minato, Y., and Yasutomo, K. (2005) Regulation of acquired immune system by notch signaling. Int J Hematol 82: 302-306.

Mitchell, T.J. (2003) The pathogenesis of streptococcal infections: from tooth decay to meningitis. Nat Rev Microbiol 1: 219-230.

Mitsos, L.M., Cardon, L.R., Fortin, A., Ryan, L., LaCourse, R., North, R.J., and Gros, P. (2000) Genetic control of susceptibility to infection with Mycobacterium tuberculosis in mice. Genes Immun 1: 467-477.

Mitsos, L.M., Cardon, L.R., Ryan, L., LaCourse, R., North, R.J., and Gros, P. (2003) Susceptibility to tuberculosis: a locus on mouse chromosome $19(\operatorname{Trl}-4)$ regulates Mycobacterium tuberculosis replication in the lungs. Proc Natl Acad Sci US A 100: 6610-6615.

Miyairi, I., Tatireddigari, V.R., Mahdi, O.S., Rose, L.A., Belland, R.J., Lu, L., Williams, R.W., et al. (2007) The p47 GTPases ligp2 and Irgb10 regulate innate immunity and inflammation to murine Chlamydia psittaci infection. J Immunol 179: 1814-1824.

Miyoshi-Akiyama, T., Takamatsu, D., Koyanagi, M., Zhao, J., Imanishi, K., and Uchiyama, T. (2005) Cytocidal effect of Streptococcus pyogenes on mouse neutrophils in vivo and the critical role of streptolysin S. J Infect Dis 192: 107-116.

Monsalve, E., Perez, M.A., Rubio, A., Ruiz-Hidalgo, M.J., Baladron, V., GarciaRamirez, J.J., Gomez, J.C., et al. (2006) Notch-1 up-regulation and signaling following macrophage activation modulates gene expression patterns known to affect antigenpresenting capacity and cytotoxic activity. J Immunol 176: 5362-5373.

Morahan, G., and Williams, R.W. (2007) Systems genetics: the next generation in genetics research? Novartis Found Symp 281: 181-188; discussion 188-191, 208-189.

Mosca, M., Polentarutti, N., Mangano, G., Apicella, C., Doni, A., Mancini, F., De Bortoli, M., et al. (2007) Regulation of the microsomal prostaglandin E synthase-1 in polarized mononuclear phagocytes and its constitutive expression in neutrophils. $J$ Leukoc Biol 82: 320-326. 
Mountz, J.D., Yang, P., Wu, Q., Zhou, J., Tousson, A., Fitzgerald, A., Allen, J., et al. (2005) Genetic segregation of spontaneous erosive arthritis and generalized autoimmune disease in the BXD2 recombinant inbred strain of mice. Scand J Immunol 61: 128-138.

Muller, M.P., Low, D.E., Green, K.A., Simor, A.E., Loeb, M., Gregson, D., and McGeer, A. (2003) Clinical and epidemiologic features of group a streptococcal pneumonia in Ontario, Canada. Arch Intern Med 163: 467-472.

Munoz-Planillo, R., Franchi, L., Miller, L.S., and Nunez, G. (2009) A critical role for hemolysins and bacterial lipoproteins in Staphylococcus aureus-induced activation of the Nlrp3 inflammasome. J Immunol 183: 3942-3948.

Nakagawa, I., Nakata, M., Kawabata, S., and Hamada, S. (2004) Transcriptome analysis and gene expression profiles of early apoptosis-related genes in Streptococcus pyogenesinfected epithelial cells. Cell Microbiol 6: 939-952.

Nakayama, T., Mutsuga, N., Yao, L., and Tosato, G. (2006) Prostaglandin E2 promotes degranulation-independent release of MCP-1 from mast cells. J Leukoc Biol 79: 95-104.

Narumiya, S., Sugimoto, Y., and Ushikubi, F. (1999) Prostanoid receptors: structures, properties, and functions. Physiol Rev 79: 1193-1226.

Nauseef, W.M. (2007) How human neutrophils kill and degrade microbes: an integrated view. Immunol Rev 219: 88-102.

Navarini, A.A., Lang, K.S., Verschoor, A., Recher, M., Zinkernagel, A.S., Nizet, V., Odermatt, B., et al. (2009) Innate immune-induced depletion of bone marrow neutrophils aggravates systemic bacterial infections. Proc Natl Acad Sci U S A 106: 7107-7112.

Nilsson, M., Sorensen, O.E., Morgelin, M., Weineisen, M., Sjobring, U., and Herwald, H. (2006) Activation of human polymorphonuclear neutrophils by streptolysin $\mathrm{O}$ from Streptococcus pyogenes leads to the release of proinflammatory mediators. Thromb Haemost 95: 982-990.

Nooh, M.M., El-Gengehi, N., Kansal, R., David, C.S., and Kotb, M. (2007) HLA transgenic mice provide evidence for a direct and dominant role of HLA class II variation in modulating the severity of streptococcal sepsis. J Immunol 178: 3076-3083.

Norrby-Teglund, A., Lustig, R., and Kotb, M. (1997) Differential induction of Th1 versus Th2 cytokines by group A streptococcal toxic shock syndrome isolates. Infect Immun 65: 5209-5215.

Norrby-Teglund, A., Chatellier, S., Low, D.E., McGeer, A., Green, K., and Kotb, M. (2000) Host variation in cytokine responses to superantigens determine the severity of invasive group A streptococcal infection. Eur J Immunol 30: 3247-3255.

Norrby-Teglund, A., and Kotb, M. (2000) Host-microbe interactions in the pathogenesis of invasive group A streptococcal infections. J Med Microbiol 49: 849-852. 
Norrby-Teglund, A., Thulin, P., Gan, B.S., Kotb, M., McGeer, A., Andersson, J., and Low, D.E. (2001) Evidence for superantigen involvement in severe group a streptococcal tissue infections. J Infect Dis 184: 853-860.

Norrby-Teglund, A., Nepom, G.T., and Kotb, M. (2002) Differential presentation of group A streptococcal superantigens by HLA class II DQ and DR alleles. Eur J Immunol 32: $2570-2577$.

Ouburg, S., Bart, A.C.J., Klinkenberg-Knol, E.C., Mulder, C.J., Salvador Pena, A., and Morre, S.A. (2005) A candidate gene approach of immune mediators effecting the susceptibility to and severity of upper gastrointestinal tract diseases in relation to Helicobacter pylori and Epstein-Barr virus infections. Eur J Gastroenterol Hepatol 17: 1213-1224.

Peirce, J.L., Chesler, E.J., Williams, R.W., and Lu, L. (2003) Genetic architecture of the mouse hippocampus: identification of gene loci with selective regional effects. Genes Brain Behav 2: 238-252.

Peirce, J.L., Lu, L., Gu, J., Silver, L.M., and Williams, R.W. (2004) A new set of BXD recombinant inbred lines from advanced intercross populations in mice. BMC Genet 5: 7.

Peirce, J.L., Broman, K.W., Lu, L., Chesler, E.J., Zhou, G., Airey, D.C., Birmingham, A.E., et al. (2008) Genome Reshuffling for Advanced Intercross Permutation (GRAIP): simulation and permutation for advanced intercross population analysis. PLoS One 3: e1977.

Peters, L.L., Zhang, W., Lambert, A.J., Brugnara, C., Churchill, G.A., and Platt, O.S. (2005) Quantitative trait loci for baseline white blood cell count, platelet count, and mean platelet volume. Mamm Genome 16: 749-763.

Peters, L.L., Robledo, R.F., Bult, C.J., Churchill, G.A., Paigen, B.J., and Svenson, K.L. (2007) The mouse as a model for human biology: a resource guide for complex trait analysis. Nat Rev Genet 8: 58-69.

Petkova, S.B., Yuan, R., Tsaih, S.W., Schott, W., Roopenian, D.C., and Paigen, B. (2008) Genetic influence on immune phenotype revealed strain-specific variations in peripheral blood lineages. Physiol Genomics 34: 304-314.

Plant, J., and Glynn, A.A. (1976) Genetics of resistance to infection with Salmonella typhimurium in mice. J Infect Dis 133: 72-78.

Proft, T., Sriskandan, S., Yang, L., and Fraser, J.D. (2003) Superantigens and streptococcal toxic shock syndrome. Emerg Infect Dis 9: 1211-1218.

Rittirsch, D., Flierl, M.A., and Ward, P.A. (2008) Harmful molecular mechanisms in sepsis. Nat Rev Immunol 8: 776-787. 
Rolink, A.G., Balciunaite, G., Demoliere, C., and Ceredig, R. (2006) The potential involvement of Notch signaling in NK cell development. Immunol Lett 107: 50-57.

Roper, R.J., Weis, J.J., McCracken, B.A., Green, C.B., Ma, Y., Weber, K.S., Fairbairn, D., et al. (2001) Genetic control of susceptibility to experimental Lyme arthritis is polygenic and exhibits consistent linkage to multiple loci on chromosome 5 in four independent mouse crosses. Genes Immun 2: 388-397.

Rosen, G.D., and Williams, R.W. (2001) Complex trait analysis of the mouse striatum: independent QTLs modulate volume and neuron number. BMC Neurosci 2: 5.

Roy, M.F., and Malo, D. (2002) Genetic regulation of host responses to Salmonella infection in mice. Genes Immun 3: 381-393.

Roy, M.F., Riendeau, N., Loredo-Osti, J.C., and Malo, D. (2006) Complexity in the host response to Salmonella Typhimurium infection in $\mathrm{AcB}$ and $\mathrm{BcA}$ recombinant congenic strains. Genes Immun 7: 655-666.

Roy, M.F., Riendeau, N., Bedard, C., Helie, P., Min-Oo, G., Turcotte, K., Gros, P., et al. (2007) Pyruvate kinase deficiency confers susceptibility to Salmonella typhimurium infection in mice. J Exp Med 204: 2949-2961.

Ruiz, N., Wang, B., Pentland, A., and Caparon, M. (1998) Streptolysin O and adherence synergistically modulate proinflammatory responses of keratinocytes to group A streptococci. Mol Microbiol 27: 337-346.

Sakata, D., Yao, C., and Narumiya, S. (2010) Prostaglandin E(2), an immunoactivator. $J$ Pharmacol Sci 112: 1-5.

Saraiva, M., and O'Garra, A. (2010) The regulation of IL-10 production by immune cells. Nat Rev Immunol 10: 170-181.

Serezani, C.H., Chung, J., Ballinger, M.N., Moore, B.B., Aronoff, D.M., and PetersGolden, M. (2007) Prostaglandin E2 suppresses bacterial killing in alveolar macrophages by inhibiting NADPH oxidase. Am J Respir Cell Mol Biol 37: 562-570.

Shifman, S., Bell, J.T., Copley, R.R., Taylor, M.S., Williams, R.W., Mott, R., and Flint, J. (2006) A high-resolution single nucleotide polymorphism genetic map of the mouse genome. PLoS Biol 4: e395.

Sieberts, S.K., and Schadt, E.E. (2007) Moving toward a system genetics view of disease. Mamm Genome 18: 389-401.

Singleton, K.D., and Wischmeyer, P.E. (2006) Effects of HSP70.1/3 gene knockout on acute respiratory distress syndrome and the inflammatory response following sepsis. $\mathrm{Am}$ J Physiol Lung Cell Mol Physiol 290: L956-961. 
Sjolinder, H., Lovkvist, L., Plant, L., Eriksson, J., Aro, H., Jones, A., and Jonsson, A.B. (2008) The ScpC protease of Streptococcus pyogenes affects the outcome of sepsis in a murine model. Infect Immun 76: 3959-3966.

Skamene, E. (1983) Genetic regulation of host resistance to bacterial infection. Rev Infect Dis 5 Suppl 4: S823-832.

Smith, R.S., Kelly, R., Iglewski, B.H., and Phipps, R.P. (2002) The Pseudomonas autoinducer $\mathrm{N}$-(3-oxododecanoyl) homoserine lactone induces cyclooxygenase-2 and prostaglandin E2 production in human lung fibroblasts: implications for inflammation. $J$ Immunol 169: 2636-2642.

Soehnlein, O., Oehmcke, S., Ma, X., Rothfuchs, A.G., Frithiof, R., van Rooijen, N., Morgelin, M., et al. (2008a) Neutrophil degranulation mediates severe lung damage triggered by streptococcal M1 protein. Eur Respir J 32: 405-412.

Soehnlein, O., Zernecke, A., Eriksson, E.E., Rothfuchs, A.G., Pham, C.T., Herwald, H., Bidzhekov, K., et al. (2008b) Neutrophil secretion products pave the way for inflammatory monocytes. Blood 112: 1461-1471.

Staali, L., Bauer, S., Morgelin, M., Bjorck, L., and Tapper, H. (2006) Streptococcus pyogenes bacteria modulate membrane traffic in human neutrophils and selectively inhibit azurophilic granule fusion with phagosomes. Cell Microbiol 8: 690-703.

Sumby, P., Porcella, S.F., Madrigal, A.G., Barbian, K.D., Virtaneva, K., Ricklefs, S.M., Sturdevant, D.E., et al. (2005) Evolutionary origin and emergence of a highly successful clone of serotype M1 group a Streptococcus involved multiple horizontal gene transfer events. J Infect Dis 192: 771-782.

Sumby, P., Zhang, S., Whitney, A.R., Falugi, F., Grandi, G., Graviss, E.A., Deleo, F.R., et al. (2008) A Chemokine-Degrading Extracellular Protease Made by Group A Streptococcus Alters Pathogenesis by Enhancing Evasion of the Innate Immune Response. Infect Immun.

Sun, H., Ringdahl, U., Homeister, J.W., Fay, W.P., Engleberg, N.C., Yang, A.Y., Rozek, L.S., et al. (2004) Plasminogen is a critical host pathogenicity factor for group A streptococcal infection. Science 305: 1283-1286.

Takahashi, E., Kuranaga, N., Satoh, K., Habu, Y., Shinomiya, N., Asano, T., Seki, S., et al. (2007) Induction of CD16+ CD56bright NK cells with antitumour cytotoxicity not only from CD16- CD56bright NK Cells but also from CD16- CD56dim NK cells. Scand J Immunol 65: 126-138.

Tapper, H., and Herwald, H. (2000) Modulation of hemostatic mechanisms in bacterial infectious diseases. Blood 96: 2329-2337.

Taylor, B. (1978) Recombinant inbred strains: Use in gene mapping. . NY: Academic Press. 
Taylor, B.A., Wnek, C., Kotlus, B.S., Roemer, N., MacTaggart, T., and Phillips, S.J. (1999) Genotyping new BXD recombinant inbred mouse strains and comparison of BXD and consensus maps. Mamm Genome 10: 335-348.

Thach, D.C., Kleeberger, S.R., Tucker, P.C., and Griffin, D.E. (2001) Genetic control of neuroadapted sindbis virus replication in female mice maps to chromosome 2 and associates with paralysis and mortality. $J$ Virol 75: 8674-8680.

Thulin, P., Johansson, L., Low, D.E., Gan, B.S., Kotb, M., McGeer, A., and NorrbyTeglund, A. (2006) Viable Group A Streptococci in Macrophages during Acute Soft Tissue Infection. PLoS Med 3: e53.

Ting, J.P., Lovering, R.C., Alnemri, E.S., Bertin, J., Boss, J.M., Davis, B.K., Flavell, R.A., et al. (2008) The NLR gene family: a standard nomenclature. Immunity 28: 285287.

Tober, K.L., Thomas-Ahner, J.M., Maruyama, T., and Oberyszyn, T.M. (2007) Possible cross-regulation of the E prostanoid receptors. Mol Carcinog 46: 711-715.

Tsaih, S.W., Lu, L., Airey, D.C., Williams, R.W., and Churchill, G.A. (2005)

Quantitative trait mapping in a diallel cross of recombinant inbred lines. Mamm Genome 16: $344-355$.

Tuite, A., Mullick, A., and Gros, P. (2004) Genetic analysis of innate immunity in resistance to Candida albicans. Genes Immun 5: 576-587.

Tuite, A., Elias, M., Picard, S., Mullick, A., and Gros, P. (2005) Genetic control of susceptibility to Candida albicans in susceptible A/J and resistant C57BL/6J mice. Genes Immun 6: 672-682.

Turnbull, I.R., Clark, A.T., Stromberg, P.E., Dixon, D.J., Woolsey, C.A., Davis, C.G., Hotchkiss, R.S., et al. (2009) Effects of aging on the immunopathologic response to sepsis. Crit Care Med 37: 1018-1023.

Valdar, W., Flint, J., and Mott, R. (2006) Simulating the collaborative cross: power of quantitative trait loci detection and mapping resolution in large sets of recombinant inbred strains of mice. Genetics 172: 1783-1797.

van den Brandt, J., Voss, K., Schott, M., Hunig, T., Wolfe, M.S., and Reichardt, H.M. (2004) Inhibition of Notch signaling biases rat thymocyte development towards the NK cell lineage. Eur J Immunol 34: 1405-1413.

Van Rooijen, N., and Sanders, A. (1994) Liposome mediated depletion of macrophages: mechanism of action, preparation of liposomes and applications. J Immunol Methods 174: 83-93. 
Virtaneva, K., Graham, M.R., Porcella, S.F., Hoe, N.P., Su, H., Graviss, E.A., Gardner, T.J., et al. (2003) Group A Streptococcus gene expression in humans and cynomolgus macaques with acute pharyngitis. Infect Immun 71: 2199-2207.

Virtaneva, K., Porcella, S.F., Graham, M.R., Ireland, R.M., Johnson, C.A., Ricklefs, S.M., Babar, I., et al. (2005) Longitudinal analysis of the group A Streptococcus transcriptome in experimental pharyngitis in cynomolgus macaques. Proc. Natl. Acad. Sci. USA 102: 9014-9019.

Vladimirov, V., Badalova, J., Svobodova, M., Havelkova, H., Hart, A.A., Blazkova, H., Demant, P., et al. (2003) Different genetic control of cutaneous and visceral disease after Leishmania major infection in mice. Infect Immun 71: 2041-2046.

Vogel, G. (2003) Scientists dream of 1001 complex mice. Science 301: 456-458.

Voyich, J.M., Musser, J.M., and DeLeo, F.R. (2004) Streptococcus pyogenes and human neutrophils: a paradigm for evasion of innate host defense by bacterial pathogens. Microbes Infect 6: 1117-1123.

Walker, M.J., Hollands, A., Sanderson-Smith, M.L., Cole, J.N., Kirk, J.K., Henningham, A., McArthur, J.D., et al. (2007) DNase Sdal provides selection pressure for a switch to invasive group A streptococcal infection. Nat Med 13: 981-985.

Wang, J., Williams, R.W., and Manly, K.F. (2003) WebQTL: web-based complex trait analysis. Neuroinformatics 1: 299-308.

Waterston, R.H., and Lindblad-Toh, K., and Birney, E., and Rogers, J., and Abril, J.F., and Agarwal, P., and Agarwala, R., et al. (2002) Initial sequencing and comparative analysis of the mouse genome. Nature 420: 520-562.

Watters, J.W., Dewar, K., Lehoczky, J., Boyartchuk, V., and Dietrich, W.F. (2001) Kif1C, a kinesin-like motor protein, mediates mouse macrophage resistance to anthrax lethal factor. Curr Biol 11: 1503-1511.

Welcher, B.C., Carra, J.H., DaSilva, L., Hanson, J., David, C.S., Aman, M.J., and Bavari, S. (2002) Lethal shock induced by streptococcal pyrogenic exotoxin A in mice transgenic for human leukocyte antigen-DQ8 and human CD4 receptors: implications for development of vaccines and therapeutics. J Infect Dis 186: 501-510.

White, E.S., Atrasz, R.G., Dickie, E.G., Aronoff, D.M., Stambolic, V., Mak, T.W., Moore, B.B., et al. (2005) Prostaglandin E(2) inhibits fibroblast migration by Eprostanoid 2 receptor-mediated increase in PTEN activity. Am J Respir Cell Mol Biol 32: $135-141$.

Williams, R.W., Gu, J., Qi, S., and Lu, L. (2001) The genetic structure of recombinant inbred mice: high-resolution consensus maps for complex trait analysis. Genome Biol 2: RESEARCH0046. 
Williams, R.W. (2006) Expression genetics and the phenotype revolution. Mamm Genome 17: 496-502.

Woolard, M.D., Wilson, J.E., Hensley, L.L., Jania, L.A., Kawula, T.H., Drake, J.R., and Frelinger, J.A. (2007) Francisella tularensis-infected macrophages release prostaglandin E2 that blocks $\mathrm{T}$ cell proliferation and promotes a Th2-like response. J Immunol 178: 2065-2074.

Yao, C., Sakata, D., Esaki, Y., Li, Y., Matsuoka, T., Kuroiwa, K., Sugimoto, Y., et al. (2009) Prostaglandin E2-EP4 signaling promotes immune inflammation through Th1 cell differentiation and Th17 cell expansion. Nat Med 15: 633-640.

Ye, Z., and Ting, J.P. (2008) NLR, the nucleotide-binding domain leucine-rich repeat containing gene family. Curr Opin Immunol 20: 3-9.

Yenari, M.A., Liu, J., Zheng, Z., Vexler, Z.S., Lee, J.E., and Giffard, R.G. (2005) Antiapoptotic and anti-inflammatory mechanisms of heat-shock protein protection. Ann $N$ Y Acad Sci 1053: 74-83.

Yong, M., Mitchell, D., Caudron, A., Toth, I., and Olive, C. (2009) Expression of maturation markers on murine dendritic cells in response to group A streptococcal lipopeptide vaccines. Vaccine 27: 3313-3318.

Zhang, L., Katz, J.M., Gwinn, M., Dowling, N.F., and Khoury, M.J. (2009a) Systemsbased candidate genes for human response to influenza infection. Infect Genet Evol 9: 1148-1157.

Zhang, W.W., and Matlashewski, G. (2004) In vivo selection for Leishmania donovani miniexon genes that increase virulence in Leishmania major. Mol Microbiol 54: 10511062.

Zhang, X., Majlessi, L., Deriaud, E., Leclerc, C., and Lo-Man, R. (2009b) Coactivation of Syk kinase and MyD88 adaptor protein pathways by bacteria promotes regulatory properties of neutrophils. Immunity 31: 761-771.

Zhang, Y., Guan, Y., Schneider, A., Brandon, S., Breyer, R.M., and Breyer, M.D. (2000) Characterization of murine vasopressor and vasodepressor prostaglandin $\mathrm{E}(2)$ receptors. Hypertension 35: 1129-1134.

Zinkernagel, A.S., Timmer, A.M., Pence, M.A., Locke, J.B., Buchanan, J.T., Turner, C.E., Mishalian, I., et al. (2008) The IL-8 protease SpyCEP/ScpC of group A Streptococcus promotes resistance to neutrophil killing. Cell Host Microbe 4: 170-178. 


\section{APPENDIX A. PRIMER SEQUENCES USED FOR QPCR AND THE RELATIVE EXPRESSION LEVELS OF CANDIDATE GENES}

This appendix contains two supplementary tables for Chapter 3:

- Table A-1. Primer sequences used in quantitative PCR assays for candidate genes.

- Table A-2. Relative expression levels of candidate gene list expressed as mean fold difference between pre- and post-infection \pm standard deviation (SD) in selected resistant and susceptible strains. 
Table A-1. Primer sequences used in quantitative PCR assays for candidate genes.

\begin{tabular}{|c|c|c|c|c|}
\hline Gene & Gene Description & $\begin{array}{l}\text { Accession } \\
\text { Number }\end{array}$ & Forward 5' & Reverse 3' \\
\hline Anapc2 & Anaphase promoting complex subunit 2 & NM_175300 & ctcaaggtggecctagagact & agagtgatgatgtcacatgtgttg \\
\hline Asb6 & Ankyrin repeat and SOCS box-containing protein 6 & NM_133346 & aggagcccctggatgatt & cagctccgtgaggaccag \\
\hline Edf1 & Endothelial differentiation-related factor 1 & NM_021519 & ccgcacaggccaagtcta & aatggaatgctgcttgttctg \\
\hline Entpd2 & Ectonucleoside triphosphate diphosphohydrolase 2 & NM_009849 & cagagaccacctgcaacca & ggtctgctgtccgggtact \\
\hline Fbxw2 & F-box and WD-40 domain protein 2 & NM_013890 & caaagtcaagtctctcttgcaca & tcccaattggccaaatctt \\
\hline Garnl3 & GTPase activating RANGAP domain-like 3 & NM_178888 & tccaccatgttgccatattc & caatgtggegcttccttt \\
\hline Gpr107 & G protein-coupled receptor 107 & NM_178760 & cctgcagctctctcaggaag & cacacccgatgtcgtcacta \\
\hline Hspa 5 & Heat shock $70 \mathrm{kD}$ protein 5 (glucose-regulated protein) & NM_022310 & ctgaggcgtatttgggaaag & cagcatctttggttgcttgtc \\
\hline Ill $\alpha$ & Interleukin 1 alpha & NM_010554.4 & ttggttaaatgacctgcaaca & gagcgctcacgaacagttg \\
\hline Illrn & Interleukin 1 receptor antagonist & NM_031167 & tgtgccaagtctggagatga & ttctttgttcttgctcagatcagt \\
\hline Mapkapl & Mitogen-activated protein kinase associated protein 1 & NM_177345 & accttgccgaggagaagag & gcgtcggactcgaagtacag \\
\hline \multirow[t]{3}{*}{ Nfatc2 } & $\begin{array}{l}\text { Nfatc2 nuclear factor of activated t-cells, cytoplasmic, } \\
\text { calcineurin-dependent } 2 *\end{array}$ & NM_010899 & caggaggacaccctgtgg & tcatctgctgtcccaatgaa \\
\hline & & NM_001037177 & & \\
\hline & & NM_001037178 & & \\
\hline Notch1 & Notch gene homolog 1 (Drosophila) & NM_008714 & tgcaactgtcctctgccata & gtagcacatggggccaac \\
\hline Noxal & NADPH oxidase activator 1 & NM_172204 & atctggagcccatggattt & gcgttgtggtcatcaggaat \\
\hline Phpt1 & Phosphohistidine phosphatase 1 & NM_029293 & cagagccaggacaggaagata & ttggctttgatcttctcagttg \\
\hline Phyhd1 & Phytanoyl-CoA dioxygenase domain containing 1 & NM_172267 & gtctggagcccagggaat & gggectttagctgtagagtgg \\
\hline Ppp2r4 & Protein phosphatase $2 \mathrm{~A}$, regulatory subunit $\mathrm{B}$ & NM_138748 & ggcagctcacagctcataga & gecttctcatccacgaaatg \\
\hline Psmd5 & Proteasome (prosome, macropain) 26S subunit, non-ATPase, 5 & NM_080554 & gacgggtgaggacgtgtta & cgtccgtgatgggtataagc \\
\hline Ptges & Prostaglandin E synthase & NM_022415 & gcacactgctggtcatcaag & acgtttcagcgcatcctc \\
\hline Ptges 2 & Prostaglandin E synthase 2 & NM_133783 & cccaggaaggagacagctt & aggtaggtcttgagggcactaat \\
\hline Rab14 & RAB14, member RAS oncogene family & NM_026697 & aggtgcgetcatggtgtat & caaccagctgcttaagtggtta \\
\hline $\operatorname{Sh} 2 d 3 c$ & $\mathrm{SH} 2$ domain containing $3 \mathrm{C}$ & NM_013781 & cagatcccagatctccactca & agcatggactcgggttacag \\
\hline
\end{tabular}


Table A-1. (Continued).

\begin{tabular}{|c|c|c|c|c|}
\hline Gene & Gene Description & $\begin{array}{l}\text { Accession } \\
\text { Number }\end{array}$ & Forward 5' & Reverse 3' \\
\hline$\overline{\text { Sirpa }}$ & Signal-regulatory protein alpha & NM_007547.2 & agggagcatgcaaaccttc & tttgatccggaggaggtaga \\
\hline Trafl & TNF receptor associated factor 1 & NM_009421 & gagcacatcctgagcttgg & tctttttgagccagggtttg \\
\hline $\operatorname{Traf} 2$ & TNF receptor associated factor 2 & NM_009422 & getccttctgcctgacca & agacacaggcagcacagttc \\
\hline$T u b b 2 c 1$ & Tubulinbeta 2c & NM_146116.1 & getcetcttctacagetgttcc & cgctgattacctcccagaact \\
\hline Ubadcl & Ubiquitin associated domain containing 1 & NM_133835 & gaacaacaaccagcagaacg & tccaattcctcaggggatg \\
\hline Urm1 & Ubiquitin related modifier 1 homolog (S. cerevisiae) & NM_026615 & gagcgaccagagctgttcat & ggcatcattaatcagcacca \\
\hline
\end{tabular}

* Nfatc2 has three transcript variants; we designed a common assay for the three transcripts, and differentiating assays for each isofrom. All assays showed similar results, shown are primers sets of common assay for the three transcript variants. 
Table A-2. Relative expression levels of candidate gene list expressed as mean fold difference between pre- and post-infection \pm standard deviation (SD) in selected resistant and susceptible strains.

\begin{tabular}{|c|c|c|c|c|c|}
\hline Gene ID & Gene & Resistant & SD & Susceptible & SD \\
\hline \multicolumn{6}{|c|}{ Genes down regulated in resistant group while up regulated in susceptible group } \\
\hline Anapc2 & Anaphase promoting complex subunit 2 & 0.551 & 0.022 & 1.475 & 0.691 \\
\hline Asb6 & Ankyrin repeat and SOCS box-containing protein 6 & 0.573 & 0.482 & 2.300 & 1.801 \\
\hline Fbxw2 2 & F-box and WD-40 domain protein 2 & 0.930 & 0.384 & 1.159 & 0.268 \\
\hline Gpr107 & G protein-coupled receptor 107 & 0.866 & 0.481 & 2.196 & 0.758 \\
\hline $\operatorname{Ill} \alpha$ & Interleukin 1 alpha & 0.739 & 0.045 & 54.687 & 0.011 \\
\hline Illrn & Interleukin 1 receptor antagonist & 0.851 & 0.384 & 55.909 & 0.274 \\
\hline Mapkap1 & Mitogen-activated protein kinase associated protein 1 & 0.732 & 0.199 & 1.189 & 0.460 \\
\hline Noxal & NADPH oxidase activator 1 & 0.270 & 0.164 & 1.086 & 0.151 \\
\hline Phpt1 & Phosphohistidine phosphatase 1 & 0.229 & 0.133 & 0.714 & 0.155 \\
\hline Ptges & Prostaglandin E synthase & 0.417 & 0.101 & 5.046 & 0.679 \\
\hline Ptges2 & Prostaglandin E synthase 2 & 0.913 & 0.553 & 1.456 & 0.122 \\
\hline Rab14 & RAB14, member RAS oncogene family & 0.704 & 0.268 & 1.411 & 0.711 \\
\hline $\operatorname{Sh} 2 d 3 c$ & $\mathrm{SH} 2$ domain containing $3 \mathrm{C}$ & 0.162 & 0.102 & 1.009 & 0.389 \\
\hline Phyhdl & Phytanoyl-CoA dioxygenase domain containing 1 & 0.806 & 0.727 & 1.288 & 0.288 \\
\hline Urm1 & Ubiquitin related modifier 1 homolog (S. cerevisiae) & 0.834 & 0.625 & 1.277 & 0.309 \\
\hline \multicolumn{6}{|c|}{ Genes down regulated in both resistant group and susceptible groups } \\
\hline$p d 2$ & Ectonucleoside triphosphate diphosphohydrolase 2 & 0.359 & 0.114 & 0.654 & 0.160 \\
\hline Edfl & Endothelial differentiation-related factor 1 & 0.350 & 0.375 & 0.470 & 0.109 \\
\hline Garnl3 & GTPase activating RANGAP domain-like 3 & 0.601 & 0.292 & 0.674 & 0.158 \\
\hline Nfatc2 & Nuclear factor of activated t-cells, cytoplasmic, calcineurin-dependent 2 & 0.178 & 0.174 & 0.693 & 0.147 \\
\hline Psmd5 & Proteasome (prosome, macropain) 26S subunit, non-ATPase, 5 & 0.644 & 0.049 & 0.963 & 0.123 \\
\hline Ppp2r4 & Protein phosphatase $2 \mathrm{~A}$, regulatory subunit $\mathrm{B}$ & 0.902 & 0.108 & 0.734 & 0.150 \\
\hline Ubacl & Ubiquitin associated domain containing 1 & 0.865 & 0.960 & 0.730 & 0.971 \\
\hline$T u b b 2 c$ & Tubulin beta $2 \mathrm{c}$ & 0.846 & 0.506 & 0.925 & 0.477 \\
\hline
\end{tabular}


Table A-2. (Continued).

\begin{tabular}{|c|c|c|c|c|c|}
\hline Gene ID & Gene & Resistant & SD & Susceptible & SD \\
\hline \multicolumn{6}{|c|}{ Genes up regulated in both resistant group and susceptible group } \\
\hline Hspa5 & Heat shock $70 \mathrm{kD}$ protein 5 (glucose-regulated protein) & 1.717 & 1.016 & 4.040 & 2.587 \\
\hline Notch1 & Notch gene homolog 1 (Drosophila) & 2.005 & 1.192 & 10.424 & 7.833 \\
\hline Traf1 & Tnf receptor-associated factor 1 & 1.826 & 0.596 & 5.995 & 0.733 \\
\hline Traf2 & Tnf receptor-associated factor 2 & 2.369 & 2.694 & 2.421 & 0.844 \\
\hline Sirpa & Signal-regulatory protein alpha & 4.092 & 6.024 & 1.607 & 1.034 \\
\hline
\end{tabular}




\section{APPENDIX B. LIST OF GENES AND TRANSCRIPTS IN THE MAPPED LOCI}

This appendix contains lists of genes and transcripts in the mapped quantitative trait loci (QTLs) associated with differential susceptibility to severe group A streptococcal (GAS) sepsis. Public access to an update list of transcripts is available online at www.genenetwork.org under BXD published phenotypes, trait ID 10836. To search for this trait ID, access the search databases page at the above link, use mice as species, BXD as the group, phenotypes as type and BXD published phenotypes as database, and then enter Abdeltawab as the search term and from the retrieved results select trait ID 10836.

This appendix contains three tables:

- Table B-1. Genes and their SNPs in mapped locus on Chr 2 between 22-34 Mb.

- Table B-2. Genes and their SNPs in mapped locus on Chr 2 between 124-150 Mb.

- Table B-3. Genes and their SNPs in mapped locus on Chr X between 50-100 Mb. 
Table B-1. Genes and their SNPs in mapped locus on Chr 2 between 22-34 Mb.

\begin{tabular}{|c|c|c|c|c|c|}
\hline Position on Chr 2 (Mb) & Gene symbol & Gene description & NM ID & SNPs & SNP Density \\
\hline 22.1588 & Myo3a & myosin IIIA & NM_148413 & 465 & 1.49 \\
\hline 22.4778 & Gad2 & glutamic acid decarboxylase 2 & NM_008078 & 211 & 2.95 \\
\hline 22.6115 & 1700092C17Rik & RIKEN cDNA $1700092 \mathrm{C} 17$ gene & AK007039 & 12 & 1.91 \\
\hline 22.6298 & Apbb1ip & amyloid beta (A4) precursor protein-binding, family $B$, member 1 interacting protein & NM_019456 & 49 & 0.48 \\
\hline 22.7472 & Pdss1 & prenyl (solanesyl) diphosphate synthase, subunit 1 & NM_019501 & 0 & 0.00 \\
\hline 22.7500 & A130006I12Rik & RIKEN cDNAA130006/12 gene & AK015028 & 0 & 0.00 \\
\hline 22.7955 & Abi1 & abl-interactor 1 & NM_001077190 & 17 & 0.17 \\
\hline 22.8470 & 2210402A03Rik & RIKEN cDNA 2210402A03 gene & AK0̄08796 & 1 & 0.51 \\
\hline 22.8820 & 4930534I15Rik & RIKEN cDNA 4930534I15 gene & AK015964 & 0 & 0.00 \\
\hline 22.9237 & Acbd5 & acyl-Coenzyme A binding domain containing 5 & NM_001102436 & 4 & 0.09 \\
\hline 22.9736 & Mastl & microtubule associated serine/threonine kinase-like & NM_025979 & 0 & 0.00 \\
\hline 23.0121 & Yme1l1 & YME1-like 1 (S. cerevisiae) & NM_013771 & 4 & 0.10 \\
\hline 23.0630 & 4931423N10Rik & RIKEN cDNA 4931423N10 gene & AK016475 & 16 & 0.27 \\
\hline 23.1768 & Nxph2 & neurexophilin 2 & NM_008752 & 7 & 0.09 \\
\hline 23.3485 & A330106F07Rik & RIKEN cDNAA330106F07 gene & AK039774 & 0 & 0.00 \\
\hline 23.3656 & Spopl & speckle-type POZ protein-like & NM_029773 & 5 & 0.08 \\
\hline 23.8584 & Hnmt & histamine $\mathrm{N}$-methyltransferase & NM_080462 & 28 & 0.60 \\
\hline 23.9272 & Tbpl2 & TATA box binding protein like 2 & NM 199059 & 26 & 1.05 \\
\hline 24.0019 & LOC215253 & similar to DEAD (Asp-Glu-Ala-Asp) box polypeptide 6 & XR_030867 & 0 & 0.00 \\
\hline 24.0087 & $\| 1 \mathrm{f} 8$ & interleukin 1 family, member 8 & NM_027163 & 18 & 2.60 \\
\hline 24.0271 & 2310066D07Rik & RIKEN cDNA 2310066D07 gene & AK010059 & 0 & 0.00 \\
\hline 24.0420 & $\| 1 \mathrm{fg}$ & interleukin 1 family, member 9 & NM_153511 & 0 & 0.00 \\
\hline 24.0709 & $\| 1 f 6$ & interleukin 1 family, member 6 & NM_019450 & 1 & 0.10 \\
\hline 24.1325 & $111 \mathrm{f5}$ & interleukin 1 family, member 5 (delta) & NM_019451 & 0 & 0.00 \\
\hline 24.1467 & $\| 1 f 10$ & interleukin 1 family, member 10 & NM_153077 & 0 & 0.00 \\
\hline 24.1924 & $\| 1 \mathrm{rn}$ & interleukin 1 receptor antagonist & NM_001039701 & 2 & 0.14 \\
\hline 24.2409 & Psd4 & pleckstrin and Sec7 domain containing 4 & NM_177611 & 53 & 2.27 \\
\hline 24.2761 & Pax8 & paired box gene 8 & NM_011040 & 60 & 1.09 \\
\hline 24.4619 & Cacna1b & calcium channel, voltage-dependent, $\mathrm{N}$ type, alpha 1B subunit & NM_001042528 & 504 & 3.21 \\
\hline 24.6463 & Ehmt1 & euchromatic histone methyltransferase 1 & NM_001012518 & 292 & 2.27 \\
\hline 24.7809 & Arrdc1 & arrestin domain containing 1 & NM_178408 & 37 & 3.77 \\
\hline 24.8053 & Zmynd19 & zinc finger, MYND domain containing 19 & NM_026021 & 27 & 2.81 \\
\hline 24.8179 & Wdr85 & WD40 repeat domain 85 & NM_026044 & 10 & 0.90 \\
\hline 24.8280 & Mrpl41 & mitochondrial ribosomal protein L41 & NM_001031808 & 1 & 0.38 \\
\hline 24.8316 & Pnpla7 & patatin-like phospholipase domain containing 7 & NM_146251 & 11 & 0.14 \\
\hline 24.9099 & Nelf & nasal embryonic LHRH factor & NM_001039386 & 0 & 0.00 \\
\hline 24.9358 & Entpd8 & ectonucleoside triphosphate diphosphohydrolase 8 & NM 028093 & 11 & 2.04 \\
\hline 24.9412 & Noxa1 & NADPH oxidase activator 1 & NM_172204 & 20 & 2.11 \\
\hline 24.9973 & D530008I23 & hypothetical protein D530008I23 & AK05201 & 0 & 0.00 \\
\hline 25.0363 & Nrarp & Notch-regulated ankyrin repeat protein & NM_025980 & 1 & 0.39 \\
\hline 25.0482 & A830007P12Rik & RIKEN cDNAA830007P12 gene & NM_146115 & 2 & 0.51 \\
\hline 25.0552 & A730008L03Rik & RIKEN cDNAA730008L03 gene & NM_021393 & 0 & 0.00 \\
\hline 25.0681 & 4933433C11Rik & RIKEN cDNA 4933433C11 gene & AK017033 & 1 & 0.48 \\
\hline 25.0743 & BC061039 & cDNA sequence $\mathrm{BC} 061039$ & NM_026624 & 0 & 0.00 \\
\hline 25.0777 & Tubb2c & tubulin, beta $2 c$ & NM_146116 & 0 & 0.00 \\
\hline 25.0844 & Slc34a3 & solute carrier family 34 (sodium phosphate), member 3 & NM_080854 & 9 & 1.69 \\
\hline 25.0900 & Gm757 & gene model $757,(\mathrm{NCBI})$ & NM_001033410 & 3 & 1.30 \\
\hline 25.0943 & 2310002J15Rik & RIKEN cDNA 2310002J15 gene & NM_026415 & 0 & 0.00 \\
\hline 25.0984 & Rnf208 & ring finger protein 208 & NM_-176834 & 2 & 1.50 \\
\hline 25.1003 & Ndor1 & NADPH dependent diflavin oxidoreductase 1 & NM_001082476 & 7 & 0.66 \\
\hline 25.1110 & C730025P13Rik & RIKEN cDNA C730025P13 gene & NM_177344 & 0 & 0.00 \\
\hline 25.1181 & C430004E15Rik & RIKEN cDNA C430004E15 gene & NM_-175286 & 0 & 0.00 \\
\hline 25.1266 & Ssna1 & Sjogren's syndrome nuclear autoantigen 1 & NM_023464 & 0 & 0.00 \\
\hline 25.1280 & Anapc2 & anaphase promoting complex subunit 2 & NM_175300 & 1 & 0.07 \\
\hline 25.1437 & 4930571C24Rik & RIKEN CDNA 4930571C24 gene & AK019803 & 1 & 0.96 \\
\hline 25.1455 & Lrrc26 & leucine rich repeat containing 26 & NM 146117 & 0 & 0.00 \\
\hline 25.1468 & E130003G02Rik & RIKEN cDNA E130003G02 gene & AK076389 & 0 & 0.00 \\
\hline 25.1478 & Grin1 & glutamate receptor, ionotropic, NMDA1 (zeta 1) & NM_008169 & 6 & 0.22 \\
\hline 25.1558 & 2900034K13Rik & RIKEN cDNA $2900034 \mathrm{~K} 13$ gene & AK019326 & 0 & 0.00 \\
\hline 25.1883 & Man1b1 & mannosidase, alpha, class 1B, member 1 & NM_001029983 & 70 & 3.60 \\
\hline 25.2078 & Dpp7 & dipeptidylpeptidase 7 & NM_031843 & 6 & 1.48 \\
\hline 25.2170 & Uap1l1 & UDP-N-acteylglucosamine pyrophosphorylase 1-like 1 & NM_001033293 & 6 & 1.45 \\
\hline 25.2278 & 2010317E24Rik & RIKEN cDNA 2010317 E24 gene & NM_001081085 & 22 & 3.73 \\
\hline 25.2514 & Entpd2 & ectonucleoside triphosphate diphosphohydrolase 2 & NM_009849 & 12 & 2.20 \\
\hline 25.2586 & Npdc1 & neural proliferation, differentiation and control gene 1 & NM_008721 & 6 & 0.94 \\
\hline 25.2788 & Fut7 & fucosyltransferase 7 & NM_013524 & 7 & 2.27 \\
\hline 25.2842 & Abca2 & ATP-binding cassette, sub-family A ( $A B C 1$ ), member 2 & NM_007379 & 111 & 5.59 \\
\hline 25.3124 & Clic3 & chloride intracellular channel 3 & NM_027085 & 4 & 2.07 \\
\hline 25.3150 & BC029214 & cDNA sequence $\mathrm{BC} 029214$ & NM_153557 & 9 & 5.60 \\
\hline 25.3222 & Ptgds & prostaglandin D2 synthase (brain) & NM_008963 & 8 & 2.63 \\
\hline 25.3464 & Lcn12 & lipocalin 12 & NM_029958 & 0 & 0.00 \\
\hline 25.3542 & C8g & complement component 8 , gamma subunit & NM_027062 & 0 & 0.00 \\
\hline 25.3563 & Fbxw5 & F-box and WD-40 domain protein 5 & NM_013908 & 3 & 0.64 \\
\hline 25.3735 & Traf2 & Tnf receptor-associated factor 2 & NM_009422 & 48 & 1.66 \\
\hline
\end{tabular}


Table B-1. (Continued).

\begin{tabular}{|c|c|c|c|c|c|}
\hline Position on $\mathrm{Chr} 2$ (Mb) & Gene symbol & Gene description & NM ID & SNPs & SNP Density \\
\hline 25.4134 & Edf1 & endothelial differentiation-related factor 1 & NM_021519 & 7 & 1.67 \\
\hline 25.4186 & Mamdc4 & MAM domain containing 4 & NM_001081199 & 21 & 2.56 \\
\hline 25.4290 & Phpt1 & phosphohistidine phosphatase 1 & NM_029293 & 0 & 0.00 \\
\hline 25.4307 & 4921530D09Rik & RIKEN cDNA 4921530D09 gene & Al429795 & 0 & 0.00 \\
\hline 25.4309 & Gm996 & gene model 996, (NCBI) & NM_001005424 & 9 & 1.92 \\
\hline 25.4385 & B230208H17Rik & RIKEN cDNA B230208H17 gene & NM_001024616 & 42 & 1.65 \\
\hline 25.4756 & Tmem141 & transmembrane protein 141 & NM_001040130 & 0 & 0.00 \\
\hline 25.4802 & Fcna & ficolin A & NM_007995 & 2 & 0.60 \\
\hline 25.5086 & Lcn8 & lipocalin 8 & NM_033145 & 0 & 0.00 \\
\hline 25.5135 & Lcn5 & lipocalin 5 & NM_001042630 & 2 & 0.54 \\
\hline 25.5323 & $\operatorname{Lcn} 6$ & lipocalin 6 & NM_177840 & 3 & 0.62 \\
\hline 25.5382 & Lcn10 & lipocalin 10 & NM_178036 & 2 & 0.60 \\
\hline 25.5556 & Lcn13 & lipocalin 13 & NM_153558 & 0 & 0.00 \\
\hline 25.5612 & A230005M16Rik & RIKEN cDNA $\wedge 230005 \mathrm{M} 16$ gene & AK038413 & 0 & 0.00 \\
\hline 25.5624 & Bmyc & brain expressed myelocytomatosis oncogene & NM_023326 & 0 & 0.00 \\
\hline 25.5925 & RP23-225D24.3 & novel lipocalin protein & NM_001099301 & 0 & 0.00 \\
\hline 25.6211 & Lcn3 & lipocalin 3 & NM_010694 & 0 & 0.00 \\
\hline 25.6325 & Lcn11 & lipocalin 11 & NM_001100455 & 0 & 0.00 \\
\hline 25.6401 & LOC620858 & hypothetical protein LOC620858 & AK076911 & 0 & 0.00 \\
\hline 25.6495 & Glt6d1 & glycosyltransferase 6 domain containing 1 & NM_001039095 & 2 & 0.09 \\
\hline 25.6787 & $\operatorname{Lcn} 9$ & lipocalin 9 & NM_029959 & 4 & 1.68 \\
\hline 25.6985 & Sohlh1 & spermatogenesis and oogenesis specific basic helix-loop-helix 1 & NM_001001714 & 14 & 3.29 \\
\hline 25.7194 & Kcnt1 & potassium channel, subfamily T, member 1 & NM_175462 & 46 & 0.88 \\
\hline 25.7580 & Camsap1 & calmodulin regulated spectrin-associated protein 1 & Al642561 & 0 & 0.00 \\
\hline 25.7789 & 6230426l18Rik & RIKEN cDNA 6230426118 gene & AK018099 & 1 & 0.68 \\
\hline 25.8392 & A230056K03Rik & RIKEN cDNAA230056K03 gene & AK038714 & 1 & 1.09 \\
\hline 25.8541 & Ubac1 & ubiquitin associated domain containing 1 & NM_133835 & 89 & 3.84 \\
\hline 25.9111 & Btbd14a & BTB (POZ) domain containing 14A & NM_001037098 & 81 & 2.20 \\
\hline 25.9589 & C330006A16RIK & RIKEN CDNA C330006A16 gene & Alb51216 & 0 & 0.00 \\
\hline 25.9941 & 1810012K08Rik & RIKEN cDNA $1810012 \mathrm{~K} 08$ gene & AK007459 & 0 & 0.00 \\
\hline 26.0557 & Lhx3 & LIM homeobox protein 3 & NM_001039653 & 12 & 1.89 \\
\hline 26.0646 & Qsox2 & quiescin Q6 sulfhydryl oxidase 2 & NM_153559 & 19 & 0.67 \\
\hline 26.0685 & LOC381355 & hypothetical LOC381355 & XM_923021 & 0 & 0.00 \\
\hline 26.1011 & C030048H21Rik & RIKEN cDNA C030048H21 gene & AK021159 & 0 & 0.00 \\
\hline 26.1272 & 4932418E24Rik & RIKEN cDNA 4932418E24 gene & NM_177841 & 1 & 0.04 \\
\hline 26.1711 & Gpsm1 & G-protein signalling modulator 1 (AGS3-like, C. elegans) & NM_153410 & 30 & 0.92 \\
\hline 26.2036 & D2Bwg1335e & DNA segment, Chr 2, Brigham \& Women's Genetics 1335 expressed & NM_026828 & 0 & 0.00 \\
\hline 26.2078 & Card9 & caspase recruitment domain family, member 9 & NM_001037747 & 0 & 0.00 \\
\hline 26.2183 & Snapc4 & small nuclear RNA activating complex, polypeptide 4 & NM_172339 & 3 & 0.17 \\
\hline 26.2383 & Sdccag3 & serologically defined colon cancer antigen 3 & NM_001085407 & 4 & 0.61 \\
\hline 26.2397 & 9430022A06Rik & RIKEN cDNA 9430022A06 gene & AKÖ20431 & 1 & 1.05 \\
\hline 26.2449 & Pmpca & peptidase (mitochondrial processing) alpha & NM_173180 & 4 & 0.51 \\
\hline 26.2518 & Inpp5e & inositol polyphosphate-5-phosphatase $\mathrm{E}$ & NM 033134 & 17 & 1.31 \\
\hline 26.2650 & Sec16a & SEC16 homolog A (S. cerevisiae) & NM_153125 & 29 & 0.81 \\
\hline 26.3015 & 0610009E02Rik & RIKEN cDNA 0610009E02 gene & AK075572 & 31 & 2.31 \\
\hline 26.3136 & Notch1 & Notch gene homolog 1 (Drosophila) & NM_008714 & 41 & 0.90 \\
\hline 26.4366 & Egfl7 & EGF-like domain 7 & NM_178444 & 15 & 1.29 \\
\hline 26.4491 & Agpat2 & 1-acylglycerol-3-phosphate 0-acyltransferase 2 & NM_026212 & 4 & 0.38 \\
\hline 26.4593 & Snhg7 & small nucleolar RNA host gene (non-protein coding) 7 & Al427062 & 0 & 0.00 \\
\hline 26.4840 & B230317C12Rik & RIKEN cDNA B230317C12 gene & NM_019833 & 0 & 0.00 \\
\hline 26.4959 & 5730588L14Rik & RIKEN cDNA 5730588L14 gene & AK030770 & 1 & 0.18 \\
\hline 26.5232 & Lcn4 & lipocalin 4 & NM_010695 & 0 & 0.00 \\
\hline 26.6980 & Abo & ABO blood group & NM_030718 & 5 & 0.22 \\
\hline 26.7463 & Surf6 & surfeit gene 6 & NM_009298 & 0 & 0.00 \\
\hline 26.7608 & Med22 & mediator complex subunit 22 & NM_001033908 & 0 & 0.00 \\
\hline 26.7663 & Rpl7a & ribosomal protein $\mathrm{L7a}$ & NM_013721 & 0 & 0.00 \\
\hline 26.7689 & Surf1 & surfeit gene 1 & NM_013677 & 2 & 0.63 \\
\hline 26.7719 & Surf2 & surfeit gene 2 & NM_013678 & 0 & 0.00 \\
\hline 26.7756 & Surf4 & surfeit gene 4 & NM_011512 & 2 & 0.14 \\
\hline 26.7896 & Gm711 & gene model 711, (NCBI) & NM_198628 & 3 & 0.15 \\
\hline 26.8091 & Rex04 & REX4, RNA exonuclease 4 homolog (S. cerevisiae) & NM_207234 & 0 & 0.00 \\
\hline 26.8289 & Adamts13 & a disintegrin-like and metallopeptidase (reprolysin type) with thrombospondin type 1 motif, 13 & NM_001001322 & 0 & 0.00 \\
\hline 26.8655 & 5930434B04Rik & RIKEN cDNA 5930434B04 gene & NM_029862 & 0 & 0.00 \\
\hline 26.8769 & Slc2a6 & solute carrier family 2 (facilitated glucose transporter), member 6 & NM_172659 & 0 & 0.00 \\
\hline 26.9172 & $1110002 \mathrm{H} 13 \mathrm{Rik}$ & RIKEN cDNA $1110002 \mathrm{H} 13$ gene & NM_025376 & 0 & 0.00 \\
\hline 26.9349 & Adamtsl2 & ADAMTS-like 2 & NM_029981 & 0 & 0.00 \\
\hline 26.9675 & C630035N08Rik & RIKEN cDNA C630035N08 gene & NM__175427 & 1 & 0.03 \\
\hline 27.0004 & $6430548 \mathrm{G} 04$ & hypothetical protein $6430548 \mathrm{G} 04$ & AK032445 & 0 & 0.00 \\
\hline 27.0210 & Dbh & dopamine beta hydroxylase & NM_138942 & 1 & 0.06 \\
\hline 27.0449 & Sardh & sarcosine dehydrogenase & NM_138665 & 2 & 0.03 \\
\hline 27.1192 & Vav2 & vav 2 oncogene & NM_009500 & 1 & 0.01 \\
\hline 27.2981 & D2Bwg1423e & DNA segment, Chr 2, Brigham \& Women's Genetics 1423 expressed & AK038857 & 0 & 0.00 \\
\hline
\end{tabular}


Table B-1. (Continued).

\begin{tabular}{|c|c|c|c|c|c|}
\hline Position on Chr 2 (Mb) & Gene symbol & Gene description & NM ID & SNPs & SNP Density \\
\hline 27.3011 & Brd3 & bromodomain containing 3 & NM_001113573 & 2 & 0.07 \\
\hline 27.3707 & Wdr5 & WD repeat domain 5 & NM_- 080848 & 0 & 0.00 \\
\hline 27.5327 & Rxra & retinoid $\mathrm{X}$ receptor alpha & NM_-011305 & 0 & 0.00 \\
\hline 27.7384 & 2810430l11Rik & RIKEN cDNA 2810430111 gene & AK019269 & 0 & 0.00 \\
\hline 27.7419 & Col5a1 & collagen, type $V$, alpha 1 & NM_015734 & 7 & 0.05 \\
\hline 27.9320 & Fcnb & ficolin B & NM_010190 & 0 & 0.00 \\
\hline 27.9510 & F730016J06Rik & RIKEN cDNA F730016J06 gene & AK036396 & 1 & 0.03 \\
\hline 28.0486 & Olfm1 & olfactomedin 1 & NM_001038612 & 0 & 0.00 \\
\hline 28.3035 & Gm347 & gene model 347, (NCBI) & NM_-001005420 & 0 & 0.00 \\
\hline 28.3175 & 1700007K13Rik & RIKEN cDNA $1700007 \mathrm{~K} 13$ gene & NM_-027040 & 0 & 0.00 \\
\hline 28.3236 & Mrps2 & mitochondrial ribosomal protein S2 & NM_-080452 & 0 & 0.00 \\
\hline 28.3533 & Gbgt1 & globoside alpha-1,3-N-acetylgalactosaminyltransferase 1 & NM_139197 & 0 & 0.00 \\
\hline 28.3687 & Ralgds & ral guanine nucleotide dissociation stimulator & NM_- 009058 & 3 & 0.08 \\
\hline 28.4113 & Cel & carboxyl ester lipase & NM_-009885 & 0 & 0.00 \\
\hline 28.4218 & Gtt3c5 & general transcription factor IIIC, polypeptide 5 & NM__148928 & 1 & 0.06 \\
\hline 28.4650 & Gfilb & growth factor independent 1B & NM_-008114 & 0 & 0.00 \\
\hline 28.4968 & Tsc1 & tuberous sclerosis 1 & NM_022887 & 0 & 0.00 \\
\hline 28.5476 & 1700026L06Rik & RIKEN cDNA 1700026L06 gene & NM_-027283 & 0 & 0.00 \\
\hline 28.5557 & 1190002A17Rik & RIKEN cDNA $1190002 A 17$ gene & NM_-001033874 & 1 & 0.01 \\
\hline 28.6811 & Gtt3c4 & general transcription factor IIIC, polypeptide 4 & NM__172977 & 0 & 0.00 \\
\hline 28.6963 & $D d x 31$ & DEAD/H (Asp-Glu-Ala-Asp/His) box polypeptide 31 & NM_001033294 & 0 & 0.00 \\
\hline 28.7632 & Barhl1 & BarH-like 1 (Drosophila) & NM_019446 & 0 & 0.00 \\
\hline 28.9074 & 1700101E01Rik & RIKEN cDNA 1700101E01 gene & NM_001085514 & 0 & 0.00 \\
\hline 28.9158 & Ttf1 & transcription termination factor 1 & NM__o09442 & 2 & 0.07 \\
\hline 28.9805 & Setx & senataxin & NM_198033 & 0 & 0.00 \\
\hline 29.0503 & Ntng2 & netrin $\mathrm{G} 2$ & NM_-133500 & 1 & 0.02 \\
\hline 29.1064 & 6530402F18Rik & RIKEN CDNA $6530402 F 18$ gene & AK018312 & 0 & 0.00 \\
\hline 29.2024 & Med27 & mediator complex subunit 27 & NM_026896 & 2 & 0.01 \\
\hline $29.4 / 52$ & Rapgef1 & Rap guanine nucleotide exchange factor (GEF) 1 & NM_001039086 & 3 & 0.02 \\
\hline 29.6316 & Trub2 & TruB pseudouridine (psi) synthase homolog 2 (E. coli) & NM_-145520 & 0 & 0.00 \\
\hline 29.6438 & Cog4 & coenzyme Q4 homolog (yeast) & NM_-178693 & 1 & 0.11 \\
\hline 29.6582 & Slc27a4 & solute carrier family 27 (fatty acid transporter), member 4 & NM_011989 & 0 & 0.00 \\
\hline 29.6829 & Urm1 & ubiquitin related modifier 1 homolog (S. cerevisiae) & NM_- 026615 & 0 & 0.00 \\
\hline 29.7242 & 2600006K01Rik & RIKEN cDNA 2600006K01 gene & AK011165 & 0 & 0.00 \\
\hline 29.7250 & Cercam & cerebral endothelial cell adhesion molecule & NM_207298 & 1 & 0.07 \\
\hline 29.7452 & Odf2 & outer dense fiber of sperm tails 2 & NM_001113213 & 0 & 0.00 \\
\hline 29.7909 & Gle1 & GLE1 RNA export mediator (yeast) & NM_- 028923 & 0 & 0.00 \\
\hline 29.8211 & Spna2 & spectrin alpha 2 & NM_001076554 & 6 & 0.09 \\
\hline 29.8871 & Wdr34 & WD repeat domain 34 & NM_-001008498 & 0 & 0.00 \\
\hline 29.9045 & 2410198J08Rik & RIKEN cDNA 2410198J08 gene & AK010838 & 0 & 0.00 \\
\hline 29.9176 & Set & SET translocation & NM_ 023871 & 0 & 0.00 \\
\hline 29.9343 & Pkn3 & protein kinase N3 & NM_-153805 & 0 & 0.00 \\
\hline 29.9465 & Zdhhc12 & zinc finger, DHHC domain containing 12 & NM 001037762 & 1 & 0.37 \\
\hline 29.9528 & Zer1 & zer-1 homolog (C. elegans) & NM_178694 & 1 & 0.04 \\
\hline 29.9894 & Tbc1d13 & TBC1 domain family, member 13 & NM_146252 & 2 & 0.11 \\
\hline 30.0270 & Endog & endonuclease $\mathrm{G}$ & NM_007931 & 0 & 0.00 \\
\hline 30.0290 & D2Wsu81e & DNA segment, Chr 2, Wayne State University 81, expressed & NM_-172660 & 0 & 0.00 \\
\hline 30.0407 & Ccbl1 & cysteine conjugate-beta lyase 1 & NM_172404 & 0 & 0.00 \\
\hline 30.0927 & 1700084E18Rik & RIKEN cDNA 1700084 E18 gene & AK006990 & 0 & 0.00 \\
\hline 30.0933 & Lrrc8a & leucine rich repeat containing $8 \mathrm{~A}$ & NM_177725 & 0 & 0.00 \\
\hline 30.1221 & Phyhd1 & phytanoyl-CoA dioxygenase domain containing 1 & NM_-172267 & 0 & 0.00 \\
\hline 30.1397 & Dolk & dolichol kinase & NM_177648 & 0 & 0.00 \\
\hline 30.1420 & Nup188 & nucleoporin 188 & NM_-198304 & 1 & 0.02 \\
\hline 30.2003 & Sh3glb2 & SH3-domain GRB2-like endophilin B2 & NM_139302 & 0 & 0.00 \\
\hline 30.2199 & 5730472N09Rik & RIKEN cDNA 5730472N09 gene & NM_-175392 & 5 & 0.24 \\
\hline 30.2479 & Dolpp1 & dolichyl pyrophosphate phosphatase 1 & NM_020329 & 11 & 1.36 \\
\hline 30.2560 & Crat & carnitine acetyltransferase & NM_- 007760 & 91 & 5.96 \\
\hline 30.2716 & Ppp2r4 & protein phosphatase $2 \Lambda$, regulatory subunit $B$ (PR 53 ) & NM_138748 & 16 & 0.50 \\
\hline 30.3282 & ler5l & immediate early response 5 -like & NM_030244 & 0 & 0.00 \\
\hline 30.4506 & Cstad & CSA-conditional, T cell activation-dependent protein & NM_- 030137 & 2 & 0.14 \\
\hline 30.4852 & 9330198N18Rik & RIKEN cDNA 9330198N18 gene & AK020395 & 14 & 0.24 \\
\hline 30.6511 & 1700001022Rik & RIKEN cDNA 1700001022 gene & NM_198000 & 0 & 0.00 \\
\hline 30.6593 & 4930527E20Rik & RIKEN cDNA 4930527E20 gene & AK019703 & 0 & 0.00 \\
\hline 30.6635 & 2610205E22Rik & RIKEN cDNA 2610205E22 gene & NM_170592 & 1 & 0.07 \\
\hline 30.6786 & Asb6 & ankyrin repeat and SOCS box-containing protein 6 & NM_133346 & 0 & 0.00 \\
\hline 30.7009 & Prrx2 & paired related homeobox 2 & NM_- 009116 & 15 & 0.42 \\
\hline 30.7450 & Ptges & prostaglandin E synthase & NM_022415 & 1 & 0.07 \\
\hline 30.8085 & Tor1b & torsin family 1 , member $B$ & NM_133673 & 1 & 0.17 \\
\hline 30.8161 & Tor1a & torsin family 1, member $\mathrm{A}$ (torsin $\mathrm{A}$ ) & NM_-144884 & 1 & 0.14 \\
\hline 30.8284 & BC005624 & cDNA sequence BC005624 & NM_144885 & 6 & 0.66 \\
\hline 30.8294 & Prdm12 & PR domain containing 12 & XM_355325 & 0 & 0.00 \\
\hline 30.8516 & Usp20 & ubiquitin specific peptidase 20 & NM_- 028846 & 6 & 0.22 \\
\hline
\end{tabular}


Table B-1. (Continued).

\begin{tabular}{|c|c|c|c|c|c|}
\hline Position on Chr 2 (Mb) & Gene symbol & Gene description & NM ID & SNPs & SNP Density \\
\hline 30.8817 & Fnbp1 & formin binding protein 1 & NM_001038700 & 81 & 0.70 \\
\hline 31.0066 & D330023K18Rik & RIKEN CDNA D330023K18 gene & AK0̈43516 & 0 & 0.00 \\
\hline 31.0079 & Gpr107 & G protein-coupled receptor 107 & NM_178760 & 124 & 1.93 \\
\hline 31.0079 & 9930033D15Rik & RIKEN cDNA 9930033D15 gene & AK036987 & 12 & 3.69 \\
\hline 31.1014 & Freq & frequenin homolog (Drosophila) & NM_019681 & 42 & 0.85 \\
\hline 31.1699 & Hmcn2 & hemicentin 2 & NM_177649 & 369 & 2.53 \\
\hline 31.3258 & Ass1 & argininosuccinate synthetase 1 & NM_007494 & 6 & 0.12 \\
\hline 31.4282 & Fubp3 & far upstream element (FUSE) binding protein 3 & NM_001033389 & 3 & 0.07 \\
\hline 31.5263 & Exosc2 & exosome component 2 & NM_144886 & 0 & 0.00 \\
\hline 31.5441 & Abl1 & $v$-abl Abelson murine leukemia oncogene 1 & NM_001112703 & 15 & 0.13 \\
\hline 31.6617 & Qrfp & pyroglutamylated RFamide peptide & NM_183424 & 0 & 0.00 \\
\hline 31.6688 & Fibcd1 & fibrinogen $\mathrm{C}$ domain containing 1 & NM_-178887 & 11 & 0.34 \\
\hline 31.7428 & Lamc3 & laminin gamma 3 & NM_011836 & 10 & 0.17 \\
\hline 31.8041 & 1110064A23Rik & RIKEN CDNA 1110064A23 gene & AK004365 & 0 & 0.00 \\
\hline 31.8058 & 2810003C17Rik & RIKEN cDNA 2810003C17 gene & NM_145144 & 0 & 0.00 \\
\hline 31.8300 & Nup214 & nucleoporin 214 & NM_172268 & 3 & 0.04 \\
\hline 31.9224 & A130092J06Rik & RIKEN cDNAA130092J06 gene & NM_-175511 & 2 & 0.12 \\
\hline 31.9379 & 1700020L05Rik & RIKEN cDNA 1700020 L05 gene & AK006171 & 0 & 0.00 \\
\hline 31.9512 & Ppapdc3 & phosphatidic acid phosphatase type 2 domain containing 3 & NM_145521 & 2 & 0.13 \\
\hline 32.0067 & 5830434P21Rik & RIKEN cDNA 5830434P21 gene & NM_-172661 & 7 & 0.08 \\
\hline 32.0828 & E030004H24Rik & RIKEN cDNA E030004H24 gene & AK086846 & 2 & 1.67 \\
\hline 32.0922 & Pomt1 & protein-O-mannosyltransferase 1 & NM_145145 & 2 & 0.11 \\
\hline 32.1105 & Uck1 & uridine-cytidine kinase 1 & NM_011675 & 0 & 0.00 \\
\hline 32.1343 & 2900010J23Rik & RIKEN cDNA 2900010J23 gene & NM_-175190 & 3 & 0.33 \\
\hline 32.1438 & Golga2 & golgi autoantigen, golgin subfamily a, 2 & NM_001080968 & 1 & 0.05 \\
\hline 32.1640 & Dnm1 & dynamin 1 & NM_010065 & 5 & 0.11 \\
\hline 32.2188 & Ciz1 & CDKN1A interacting zinc finger protein 1 & NM_028412 & 2 & 0.13 \\
\hline 32.2346 & 1110008P14Rik & RIKEN cDNA $1110008 \mathrm{P} 14$ gene & NM_198001 & 0 & 0.00 \\
\hline 32.2402 & Lcn2 & lipocalin 2 & NM_008491 & 0 & 0.00 \\
\hline 32.2518 & Ptges2 & prostaglandin $\mathrm{E}$ synthase 2 & NM_-133783 & 0 & 0.00 \\
\hline 32.2700 & Slc25a25 & solute carrier family 25 (mitochondrial carrier, phosphate carrier), member 25 & NM_146118 & 2 & 0.05 \\
\hline 32.3060 & 4933440H19Rik & RIKEN cDNA 4933440H19 gene & NM_194335 & 0 & 0.00 \\
\hline 32.3909 & C230093N12Rik & RIKEN cDNA C230093N12 gene & NM_-153560 & 13 & 0.38 \\
\hline 32.4264 & Dpm2 & dolichol-phosphate (beta-D) mannosyltransferase 2 & NM_010073 & 3 & 1.11 \\
\hline 32.4297 & 9430097D07Rik & RIKEN cDNA 9430097D07 gene & AK035182 & 0 & 0.00 \\
\hline 32.4313 & Pip5k11 & phosphatidylinositol-4-phosphate 5-kinase-like 1 & NM_198191 & 33 & 4.15 \\
\hline 32.4430 & St6galnac4 & ST6 (alpha-N-acetyl-neuraminyl-2,3-beta-galactosyl-1,3)-N-acetylgalactosaminide alpha-2,6-sialy|transferase 4 & NM_011373 & 15 & 1.16 \\
\hline 32.4552 & St6galnac6 & ST6 (alpha-N-acetyl-neuraminyl-2,3-beta-galactosyl-1,3)-N-acetylgalactosaminide alpha-2,6-sialyltransferase 6 & NM_001025310 & 8 & 0.38 \\
\hline 32.4850 & Ak1 & adenylate kinase 1 & NM_021515 & 0 & 0.00 \\
\hline 32.5021 & Eng & endoglin & NM_007932 & 2 & 0.06 \\
\hline 32.5381 & Fpgs & folylpolyglutamyl synthetase & NM_010236 & 0 & 0.00 \\
\hline 32.5549 & 6330409D20Rik & RIKEN cDNA 6330409D20 gene & AA517851 & 0 & 0.00 \\
\hline 32.5613 & Cdk9 & cyclin-dependent kinase 9 (CDC2-related kinase) & NM_130860 & 0 & 0.00 \\
\hline 32.5766 & Sh2d3c & SH2 domain containing $3 \mathrm{C}$ & NM_013781 & 0 & 0.00 \\
\hline 32.6128 & Tor2a & torsin family 2 , member A & NM_152800 & 2 & 0.40 \\
\hline 32.6177 & Ttc16 & tetratricopeptide repeat domain 16 & NM_177384 & 3 & 0.23 \\
\hline 32.6313 & Ptrh1 & peptidyl-tRNA hydrolase 1 homolog (S. cerevisiae) & NM_178595 & 1 & 0.56 \\
\hline 32.6329 & 1700019L03Rik & RIKEN CDNA $1700019 L 03$ gene & NM_025619 & 5 & 0.72 \\
\hline 32.6431 & Stxbp1 & syntaxin binding protein 1 & NM_001113569 & 8 & 0.13 \\
\hline 32.7317 & 9130404D14Rik & RIKEN cDNA 9130404D14 gene & NM_-146119 & 11 & 0.22 \\
\hline 32.7411 & D130056L21Rik & RIKEN cDNA D130056L21 gene & AK051447 & 0 & 0.00 \\
\hline 32.7807 & Lrsam1 & leucine rich repeat and sterile alpha motif containing 1 & NM_199302 & 9 & 0.25 \\
\hline 32.7853 & Snora65 & small nucleolar RNA, H/ACA box 65 & NR_002898 & 0 & 0.00 \\
\hline 32.8172 & Rpl12 & ribosomal protein L12 & NM_009076 & 0 & 0.00 \\
\hline 32.8285 & Sic2a8 & solute carrier family 2, (facilitated glucose transporter), member 8 & NM_019488 & 0 & 0.00 \\
\hline 32.8419 & Garnl3 & GTPase activating RANGAP domain-like 3 & NM_-178888 & 12 & 0.12 \\
\hline 32.9925 & Ralgps1 & Ral GEF with $\mathrm{PH}$ domain and $\mathrm{SH} 3$ binding motif 1 & NM_175211 & 70 & 0.30 \\
\hline 33.0715 & Angpt12 & angiopoietin-like 2 & NM_011923 & 14 & 0.44 \\
\hline 33.2616 & Zbtb34 & zinc finger and BTB domain containing 34 & NM_001085507 & 11 & 0.44 \\
\hline 33.3058 & Zbtb43 & zinc finger and BTB domain containing 43 & NM_001025594 & 43 & 2.36 \\
\hline 33.4202 & $\operatorname{Lm} \times 1 b$ & LIM homeobox transcription factor 1 beta & NM_010725 & 64 & 0.84 \\
\hline 33.4969 & C130021120Rik & Riken cDNA C130021120 gene & AK081497 & 27 & 5.38 \\
\hline 33.5719 & 9430024E24Rik & RIKEN cDNA 9430024E24 gene & AK020435 & 0 & 0.00 \\
\hline 33.5855 & 2610528K11Rik & RIKEN cDNA 2610528K11 gene & NM_175184 & 266 & 1.68 \\
\hline 33.6355 & A630071L07Rik & RIKEN cDNA A630071L07 gene & AA874387 & 0 & 0.00 \\
\hline 33.8738 & C230014012Rik & RIKEN cDNA C230014012 gene & AK048710 & 151 & 1.69 \\
\hline 34.0274 & $\mathrm{~Pb} \times 3$ & pre B-cell leukemia transcription factor 3 & NM_016768 & 17 & 0.08 \\
\hline 34.1433 & C79798 & expressed sequence $\mathrm{C} 79798$ & AK138624 & 1 & 0.06 \\
\hline 34.1940 & B930068K11Rik & RIKEN cDNA B930068K11 gene & AK081020 & 0 & 0.00 \\
\hline 34.2713 & 5830434F19Rik & RIKEN cDNA 5830434 F19 gene & AK017969 & 0 & 0.00 \\
\hline 34.2875 & Mapkap1 & mitogen-activated protein kinase associated protein 1 & NM_177345 & 386 & 2.00 \\
\hline 34.3581 & 4930414H07Rik & RIKEN cDNA 4930414H07 gene & AK015137 & 1 & 1.23 \\
\hline 34.4640 & 1700007J24Rik & RIKEN cDNA $1700007 J 24$ gene & AK005727 & 0 & 0.00 \\
\hline 34.5325 & Gapvd1 & GTPase activating protein and VPS9 domains 1 & NM_025709 & 3 & 0.04 \\
\hline 34.6276 & Hspa5 & heat shock protein 5 & NM_022310 & 1 & 0.23 \\
\hline 34.6342 & Rabepk & Rab9 effector protein with kelch motifs & NM_145522 & 4 & 0.19 \\
\hline 34.6578 & 4930550L05Rik & RIKEN cDNA 4930550L05 gene & AK019757 & 2 & 0.21 \\
\hline 34.6607 & Fbxw2 & F-box and WD-40 domain protein 2 & NM_013890 & 3 & 0.14 \\
\hline 34.7076 & Psmd5 & proteasome (prosome, macropain) 26S subunit, non-ATPase, 5 & NM_080554 & 1 & 0.05 \\
\hline 34.7266 & 5730407M17Rik & RIKEN cDNA 5730407M17 gene & AK017517 & 0 & 0.00 \\
\hline 34.7300 & D730039F16Rik & RIKEN cDNA D730039F16 gene & NM_030021 & 0 & 0.00 \\
\hline 34.7493 & Phf19 & PHD finger protein 19 & NM_028716 & 16 & 0.79 \\
\hline 34.7988 & Traf1 & Tnf receptor-associated factor 1 & NM_009421 & 24 & 1.30 \\
\hline 34.8389 & $\mathrm{Hc}$ & hemolytic complement & NM_010406 & 62 & 0.79 \\
\hline 34.9396 & Al182371 & expressed sequence Al182371 & NM_178885 & 1 & 0.06 \\
\hline
\end{tabular}


Table B-2. Genes and their SNPs in mapped locus on Chr 2 between 124-150 Mb.

\begin{tabular}{|c|c|c|c|c|c|}
\hline Position on Chr 2 (Mb) & Gene symbol & Gene description & NM ID & SNPs & SNP Density \\
\hline 124.9783 & Slc12a1 & solute carrier family 12, member 1 & NM_001079690 & 224 & 2.89 \\
\hline 125.0730 & Dut & deoxyuridine triphosphatase & NM_023595 & 79 & 6.69 \\
\hline 125.1003 & A530010F05Rik & RIKEN cDNAA530010F05 gene & AK079928 & 0 & 0.00 \\
\hline 125.1263 & Fbn1 & fibrillin 1 & NM_007993 & 706 & 3.43 \\
\hline 125.3888 & Cep152 & centrosomal protein 152 & NM_001081091 & 232 & 3.74 \\
\hline 125.4532 & Shc4 & SHC (Src homology 2 domain containing) family, member 4 & NM_199022 & 353 & 3.65 \\
\hline 125.4988 & Eid1 & EP300 interacting inhibitor of differentiation 1 & NM_025613 & 13 & 5.11 \\
\hline 125.5627 & 3110001I20Rik & RIKEN cDNA 3110001120 gene & NM_177608 & 138 & 3.01 \\
\hline 125.6560 & Cops2 & COP9 (constitutive photomorphogenic) homolog, subunit 2 (Arabidopsis thaliana) & NM_009939 & 172 & 5.99 \\
\hline 125.6850 & Galk2 & galactokinase 2 & NM_175154 & 359 & 2.87 \\
\hline 125.8094 & 4930525F21Rik & RIKEN cDNA 4930525F21 gene & NM_029455 & 373 & 2.29 \\
\hline 125.8605 & Fgf7 & fibroblast growth factor 7 & NM_008008 & 139 & 2.49 \\
\hline 125.9779 & Dtwd1 & DTW domain containing 1 & NM_026981 & 76 & 5.79 \\
\hline 126.1467 & Atp8b4 & ATPase, class I, type 8B, member 4 & NM_001080944 & 593 & 3.48 \\
\hline 126.3617 & A630026N12Rik & RIKEN cDNAA630026N12 gene & $\mathrm{Al} 0 \overline{2} 1452$ & 0 & 0.00 \\
\hline 126.3788 & Slc27a2 & solute carrier family 27 (fatty acid transporter), member 2 & NM_011978 & 116 & 3.29 \\
\hline 126.4194 & $\mathrm{Hdc}$ & histidine decarboxylase & NM_-008230 & 190 & 7.60 \\
\hline 126.4546 & Gabpb1 & GA repeat binding protein, beta 1 & NM_010249 & 136 & 2.92 \\
\hline 126.5331 & Usp8 & ubiquitin specific peptidase 8 & NM_019729 & 130 & 2.50 \\
\hline 126.5349 & Polr2l & polymerase (RNA) II (DNA directed) polypeptide L & AK0̄11021 & 0 & 0.00 \\
\hline 126.5872 & Usp50 & ubiquitin specific peptidase 50 & NM_029163 & 57 & 2.59 \\
\hline 126.6173 & Trpm7 & transient receptor potential cation channel, subfamily M, member 7 & NM_021450 & 209 & 2.47 \\
\hline 126.6692 & B930086H10Rik & RIKEN cDNA B930086H10 gene & AK047541 & 12 & 7.27 \\
\hline 126.7199 & 2010106G01Rik & RIKEN cDNA $2010106 \mathrm{G} 01$ gene & NM_023220 & 132 & 3.38 \\
\hline 126.8344 & Ap4e1 & adaptor-related protein complex AP-4, epsilon 1 & NM_175550 & 159 & 2.60 \\
\hline 126.8964 & Blvra & biliverdin reductase $\mathrm{A}$ & NM_-026678 & 106 & 4.01 \\
\hline 126.9296 & Ncaph & non-SMC condensin I complex, subunit $\mathrm{H}$ & NM_-144818 & 151 & 5.02 \\
\hline 126.9663 & 1700041B20Rik & RIKEN cDNA $1700041 \mathrm{~B} 20$ gene & AK0̄06667 & 8 & 3.88 \\
\hline 127.0303 & 1810024B03Rik & RIKEN cDNA 1810024B03 gene & AK019023 & 7 & 1.92 \\
\hline 127.0341 & Ascc3l1 & activating signal cointegrator 1 complex subunit 3-like 1 & NM_177214 & 158 & 4.93 \\
\hline 127.0667 & Ciao1 & cytosolic iron-sulfur protein assembly 1 homolog (S. cerevisiae) & NM_025296 & 16 & 2.33 \\
\hline 127.0737 & Tmem127 & transmembrane protein 127 & NM_175145 & 35 & 2.74 \\
\hline 127.0960 & Stard7 & START domain containing 7 & NM_139308 & 100 & 3.48 \\
\hline 127.1619 & Dusp2 & dual specificity phosphatase 2 & NM_010090 & 9 & 4.06 \\
\hline 127.1644 & Astl & astacin-like metalloendopeptidase (M12 family) & NM_172539 & 73 & 3.84 \\
\hline 127.1890 & Adra2b & adrenergic receptor, alpha $2 b$ & NM_009633 & 15 & 3.81 \\
\hline 127.2509 & A530057A03Rik & RIKEN cDNAA530057A03 gene & NM_001081089 & 36 & 3.30 \\
\hline 127.2620 & Fahd2a & fumarylacetoacetate hydrolase domain containing $2 \mathrm{~A}$ & NM_029629 & 41 & 4.95 \\
\hline 127.2822 & Kcnip3 & Kv channel interacting protein 3 , calsenilin & NM_001111331 & 158 & 6.08 \\
\hline 127.3527 & Prom2 & prominin 2 & NM_138750 & 29 & 2.00 \\
\hline 127.4013 & Zfp661 & zinc finger protein 661 & NM_001111029 & 12 & 1.31 \\
\hline 127.4132 & Mrps5 & mitochondrial ribosomal protein S5 & NM_029963 & 40 & 2.42 \\
\hline 127.4590 & Mal & myelin and lymphocyte protein, T-cell differentiation protein & NM_010762 & 87 & 3.71 \\
\hline 127.4837 & 1500011K16Rik & RIKEN cDNA $1500011 \mathrm{~K} 16$ gene & AA738625 & 0 & 0.00 \\
\hline 127.5301 & Mall & mal, T-cell differentiation protein-like & NM_145532 & 91 & 3.57 \\
\hline 127.5665 & Nphp1 & nephronophthisis 1 (juvenile) homolog (human) & NM_016902 & 157 & 3.26 \\
\hline 127.6259 & Bub1 & budding uninhibited by benzimidazoles 1 homolog (S. cerevisiae) & NM_001113179 & 119 & 3.76 \\
\hline 127.6804 & Acoxl & acyl-Coenzyme A oxidase-like & NM_028765 & 1228 & 4.56 \\
\hline 127.7460 & 0610042E11Rik & RIKEN cDNA 0610042 E11 gene & AK0̄02906 & 11 & 4.31 \\
\hline 127.9518 & Bcl2/11 & BCL2-like 11 (apoptosis facilitator) & NM_009754 & 193 & 5.29 \\
\hline 128.1244 & LOC100043424 & hypothetical protein LOC100043424 & AK144927 & 416 & 3.18 \\
\hline 128.2881 & Gm355 & gene model $355,(\mathrm{NCBI})$ & XM_001003757 & 0 & 0.00 \\
\hline 128.4358 & Anapc1 & anaphase promoting complex subunit 1 & NM_-008569 & 305 & 3.95 \\
\hline 128.5247 & Mertk & c-mer proto-oncogene tyrosine kinase & NM_008587 & 543 & 5.26 \\
\hline 128.6440 & Tmem87b & transmembrane protein $87 \mathrm{~B}$ & NM_028248 & 83 & 2.31 \\
\hline 128.6897 & Fbln7 & fibulin 7 & NM_024237 & 114 & 3.44 \\
\hline 128.7165 & LOC215539 & similar to CG3299-PA & XM_917820 & 0 & 0.00 \\
\hline 128.7520 & Zc3h8 & zinc finger $\mathrm{CCCH}$ type containing 8 & NM_020594 & 74 & 4.17 \\
\hline 128.7814 & 4933427J07Rik & RIKEN cDNA 4933427J07 gene & AK016956 & 7 & 3.19 \\
\hline 128.7931 & Zc3h6 & zinc finger $\mathrm{CCCH}$ type containing 6 & NM_178404 & 197 & 3.85 \\
\hline 128.8643 & 4930402C16Rik & RIKEN cDNA 4930402C16 gene & AK015044 & 0 & 0.00 \\
\hline 128.8917 & Ttl & tubulin tyrosine ligase & NM_027192 & 90 & 3.24 \\
\hline 128.9268 & Rpo1-2 & RNA polymerase 1-2 & NM_009086 & 69 & 2.70 \\
\hline 128.9556 & Chchd5 & coiled-coil-helix-coiled-coil-helix domain containing 5 & NM_025395 & 20 & 4.67 \\
\hline 129.0245 & Slc20a1 & solute carrier family 20 , member 1 & NM_015747 & 0 & 0.00 \\
\hline 129.0317 & 9830144P21Rik & RIKEN cDNA 9830144P21 gene & AK0̈36644 & 0 & 0.00 \\
\hline 129.0447 & A730036l17Rik & RIKEN cDNAA730036I17 gene & AK042900 & 0 & 0.00 \\
\hline 129.0946 & Ckap2l & cytoskeleton associated protein 2-like & NM_181589 & 0 & 0.00 \\
\hline 129.1253 & II1a & interleukin 1 alpha & NM_010554 & 1 & 0.10 \\
\hline 129.1903 & II1b & interleukin 1 beta & NM_008361 & 0 & 0.00 \\
\hline 129.2841 & F830045P16Rik & RIKEN cDNA F830045P16 gene & NM_177653 & 3 & 0.04 \\
\hline 129.4193 & Sirpa & signal-regulatory protein alpha & NM_007547 & 1 & 0.03 \\
\hline 129.4221 & 2900076A13Rik & RIKEN cDNA $2900076 \mathrm{~A} 13$ gene & AK013795 & 0 & 0.00 \\
\hline 129.5137 & Pdyn & prodynorphin & NM 018863 & 1 & 0.54 \\
\hline
\end{tabular}


Table B-2. (Continued).

\begin{tabular}{|c|c|c|c|c|c|}
\hline Position on Chr 2 (Mb) & Gene symbol & Gene description & NM ID & SNPS & SNP Density \\
\hline 129.8381 & Tgm3 & transglutaminase 3 , E polypeptide & $\mathrm{NM}^{-} 009374$ & 1 & 0.03 \\
\hline 129.9261 & AU015228 & expressed sequence AU015228 & NM_001033197 & 0 & 0.00 \\
\hline 129.9974 & Snrpb & small nuclear ribonucleoprotein B & NM_009225 & 0 & 0.00 \\
\hline 130.0209 & Tmc2 & transmembrane channel-like gene family 2 & NM_138655 & 4 & 0.06 \\
\hline 130.0995 & 2810036E18Rik & RIKEN cDNA 2810036 E18 gene & AK012862 & 0 & 0.00 \\
\hline 130.1105 & A930025D01Rik & RIKEN cDNAA930025D01 gene & NM_001114541 & 0 & 0.00 \\
\hline 130.1217 & Ebf4 & early B-cell factor 4 & NM_001110513 & 2 & 0.03 \\
\hline 130.2138 & C130063K03Rik & RIKEN cDNA C130063K03 gene & AK048471 & 1 & 0.60 \\
\hline 130.2165 & Cpxm1 & carboxypeptidase X 1 (M14 family) & NM_019696 & 0 & 0.00 \\
\hline 130.2246 & $\mathrm{C} 230006 \mathrm{~B} 20$ & hypothetical LOC403344 & AK0-82093 & 0 & 0.00 \\
\hline 130.2312 & 1700020A23Rik & RIKEN cDNA 1700020A23 gene & AK006141 & 0 & 0.00 \\
\hline 130.3497 & 9530056E24Rik & RIKEN cDNA 9530056 E24 gene & AK035489 & 0 & 0.00 \\
\hline 130.3695 & 4930473A02Rik & RIKEN cDNA 4930473A02 gene & AK015562 & 0 & 0.00 \\
\hline 130.3895 & Mrps26 & mitochondrial ribosomal protein S26 & NM_207207 & 0 & 0.00 \\
\hline 130.4019 & Oxt & oxytocin & NM_011025 & 0 & 0.00 \\
\hline 130.4064 & Avp & arginine vasopressin & NM_009732 & 0 & 0.00 \\
\hline 130.4157 & Ubox5 & U box domain containing 5 & NM_080562 & 2 & 0.05 \\
\hline 130.4396 & Fastkd5 & FAST kinase domains 5 & NM_198176 & 0 & 0.00 \\
\hline 130.4586 & RP23-100C5.8 & ProSAPiP1 protein & NM_197945 & 0 & 0.00 \\
\hline 130.4798 & 2600009E05Rik & RIKEN cDNA 2600009E05 gene & NM_029832 & 0 & 0.00 \\
\hline 130.4936 & Itpa & inosine triphosphatase (nucleoside triphosphate pyrophosphatase) & NM_025922 & 1 & 0.07 \\
\hline 130.5098 & SIc4a11 & solute carrier family 4 , sodium bicarbonate transporter-like, member 11 & NM_001081162 & 0 & 0.00 \\
\hline 130.5336 & 4930402H24Rik & RIKEN cDNA $4930402 \mathrm{H} 24$ gene & NM_029432 & 5 & 0.04 \\
\hline 130.6659 & C030014O09Rik & RIKEN cDNA C030014009 gene & AK021082 & 0 & 0.00 \\
\hline 131.0030 & Cenpb & centromere protein $\mathrm{B}$ & NM_007682 & 14 & 5.14 \\
\hline 131.0127 & Cdc25b & cell division cycle 25 homolog B (S. pombe) & NM_0011111075 & 63 & 5.46 \\
\hline 131.0362 & 2310035K24Rik & RIKEN cDNA $2310035 \mathrm{~K} 24$ gene & NM_027129 & 2 & 0.65 \\
\hline 131.0599 & D430028G21Rik & RIKEN cDNA D430028G21 gene & NM_144888 & 30 & 2.16 \\
\hline 131.0882 & Pank2 & pantothenate kinase 2 (Hallervorden-Spatz syndrome) & NM_153501 & 125 & 3.41 \\
\hline 131.1242 & Rnf24 & ring finger protein 24 & NM_-178607 & 141 & 2.59 \\
\hline 131.3177 & Smox & spermine oxidase & NM_145533 & 116 & 3.49 \\
\hline 131.3711 & Adra1d & adrenergic receptor, alpha $1 \mathrm{~d}$ & NM_013460 & 34 & 2.01 \\
\hline 131.4736 & 4930425F17Rik & RIKEN cDNA 4930425F17 gene & AK019583 & 0 & 0.00 \\
\hline 131.5470 & Erv3 & endogenous retroviral sequence 3 & AA087213 & 0 & 0.00 \\
\hline 131.6457 & 5330413P13Rik & RIKEN cDNA $5330413 \mathrm{P} 13$ gene & AK030453 & 72 & 2.60 \\
\hline 131.7357 & Prnp & prion protein & NM_011170 & 2 & 0.07 \\
\hline 131.7766 & Prnd & prion protein dublet & NM_023043 & 0 & 0.00 \\
\hline 131.8186 & Rassf2 & Ras association (RalGDS/AF-6) domain family 2 & NM_175445 & 3 & 0.08 \\
\hline 131.8782 & Slc23a2 & solute carrier family 23 (nucleobase transporters), member 2 & NM_018824 & 12 & 0.13 \\
\hline 131.9064 & EG329521 & predicted gene, EG329521 & XM_001480655 & 0 & 0.00 \\
\hline 132.0652 & 5730494N06Rik & RIKEN cDNA 5730494 N06 gene & NM_027478 & 0 & 0.00 \\
\hline 132.0750 & Pcna & proliferating cell nuclear antigen & NM_011045 & 0 & 0.00 \\
\hline 132.0890 & Cds2 & CDP-diacylglycerol synthase (phosphatidate cytidylyltransferase) 2 & NM_138651 & 2 & 0.04 \\
\hline 132.1969 & Prokr2 & prokineticin receptor 2 & NM_144944 & 0 & 0.00 \\
\hline 132.2505 & 4921508D12Rik & RIKEN cDNA 4921508D12 gene & AK014842 & 3 & 0.11 \\
\hline 132.3154 & 1700058J15Rik & RIKEN cDNA $1700058 \mathrm{~J} 15$ gene & AK006832 & 0 & 0.00 \\
\hline 132.3165 & 1700026D11Rik & RIKEN cDNA 1700026D11 gene & AK006376 & 2 & 0.06 \\
\hline 132.3548 & Prei4 & preimplantation protein 4 & NM_001042671 & 1 & 0.02 \\
\hline 132.3847 & E030016H06Rik & RIKEN cDNA E030016H06 gene & AK0̈86966 & 0 & 0.00 \\
\hline
\end{tabular}


Table B-2. (Continued).

Position on Chr 2 (Mb) Gene symbol Gene description

NM ID

327770

133.2578

133.3789

134.3231

134.4202

134.6119

134.9953

135.4063

135.5676

135.8837

135.9068

135.9460

136.3276

136.3581

136.5392

136.6995

1367170

136.9072

138.0823

139.0826

139.3197

139.4499

139.4991

139.6592

139.7646

139.8899

139.9456

139.9964

140.0556

140.2212

140.4839

141.0008

1424441

142.7287

142.8888

142.9042

143361

143.3719

143.5733

143.6523

143.7411

143.7731

143.8588

143.9989

1440690

144.0759

144.0968

1441309

144.1575

144.1948

144.2851

144.2909

144.2963

144.3535

144.3680

1443820

1444198

144.4257

144.6494

145.0683

145.5013

145.5013

145.6119

145.7290

145.7319

145.7432

145.7605

46.0477

146.0670

146.0850

146.0958

462274

146.3687

5830467P10Rik RIKEN cDNA 5830467P10 gene

2900022B07Rik RIKEN cDNA 2900022B07 gene

A430048G15Rik RIKEN cDNA A430048G15 gene

Bmp2 bone morphogenetic protein 2

Hao1 hydroxyacid oxidase 1, liver

Txndc13 thioredoxin domain containing 13

Plcb1 phospholipase $\mathrm{C}$, beta 1

4930545L23Rik RIKEN cDNA 4930545L23 gene

9630028H03Rik RIKEN cDNA 9630028H03 gene

Plcb4 phospholipase $\mathrm{C}$, beta 4

6330527006Rik RIKEN cDNA 6330527006 gene

Pak7 p21 (CDKN1A)-activated kinase 7

2900018K06Rik RIKEN cDNA 2900018K06 gene

cDNA sequence BC034902

Ankrd5 ankyrin repeat domain 5

Snap25 synaptosomal-associated protein 25

Mkks McKusick-Kaufman syndrome protein

2210009G21Rik RIKEN cDNA 2210009G21 gene

Jag1 jagged 1

Btbd3 BTB (POZ) domain containing 3

LOC545466 hypothetical LOC545466

Sptlc3 serine palmitoyltransferase, long chain base subunit 3

5430433G21Rik RIKEN cDNA 5430433G21 gene

3021401L19Rik RIKEN cDNA 3021401L19 gene

Tasp1 taspase, threonine aspartase 1

LOC269365 similar to ribosomal protein S19

LOC672103 hypothetical LOC672103

Esf1 ESF1, nucleolar pre-rRNA processing protein, homolog (S. cerevisiae)

2310003L22Rik RIKEN cDNA 2310003L22 gene

Sel1/2 sel-1 suppressor of lin-12-like 2 (C. elegans)

Macrod2 MACRO domain containing 2

Flrt3 fibronectin leucine rich transmembrane protein 3

2900060K15Rik RIKEN cDNA 2900060K15 gene

Kif16b kinesin family member 16B

4930511F01Rik RIKEN cDNA 4930511F01 gene

Snrpb2 U2 small nuclear ribonucleoprotein B

Otor otoraplin

C630020P19Rik RIKEN cDNA C630020P19 gene

Pcsk2 proprotein convertase subtilisin/kexin type 2

9430032N09Rik RIKEN cDNA 9430032N09 gene

Bfsp1 beaded filament structural protein in lens-CP94

Dstn destrin

Rrbp1 ribosome binding protein 1

Banf2 barrier to autointegration factor 2

EG433481 predicted gene, EG433481

4930444E06Rik RIKEN cDNA 4930444E06 gene

Snx5 Sorting nexin 5

8430406l07Rik RIKEN cDNA 8430406107 gene

Ovol2 ovo-like 2 (Drosophila)

1700108N11Rik RIKEN cDNA 1700108N11 gene

Csrp2bp cysteine and glycine-rich protein 2 binding protein

LOC668917 similar to Zinc finger protein 133

Zfp133 zinc finger protein 133

6330439K17Rik RIKEN cDNA 6330439K17 gene

Polr3f polymerase (RNA) III (DNA directed) polypeptide F

Rbbp9 retinoblastoma binding protein 9

Sec23b SEC23B (S. cerevisiae)

Gm561 gene model 561, (NCBI)

Dtd1 D-tyrosyl-tRNA deacylase 1 homolog (S. cerevisiae)

1700010M22Rik RIKEN cDNA 1700010M22 gene

SIc24a3

BC039771

hypothetical gene supported by BC02855

N-acetyltransferase 5 (ARD1 homolog S. cerevisiae)

AU022840 expressed sequence AU022840

Crnkl1 Crn, crooked neck-like 1 (Drosophila)

4930529M08Rik RIKEN cDNA 4930529M08 gene

Insm1 insulinoma-associated 1

A230067G21Rik RIKEN cDNA A230067G21 gene

A930019D19Rik RIKEN cDNA A930019D19 gene

B130033B12Rik RIKEN cDNA B130033B12 gene

9630019E01Rik RIKEN cDNA 9630019E01 gene

4933406D12Rik RIKEN cDNA 4933406D12 gene
NM 19802

AK013567

AK040035

NM_007553

NM_010403

NM_029148

NM 019677

AK019742

AK036032

NM_013829

NM 029530

NM_172858

AK0 13551

AK040732

NM 175667

NM_011428

NM_021527

NM_00103864

NM 013822

NM_001025431

AK090153

NM 175467

Al849658

AK013907

NM_175225

XM_001005

AK139706

NM_001081090

NM_027093

NM 001033296

NM 001013802

NM_178382

AK013735

NM 001081133

AK015749

NM_021335

NM 020595

AK035754

NM_008792

AK0̈34766

NM 009751

NM 019771

NM_024281

NM_001044750

AK144672

AK015377

NM_024225

NM_028984

NM 026924

AK007144

NM 181417

AK137157

AF332089

NM 172859

NM_029763

NM_015754

NM 019787

NM_001033297

NM_025314

NM_025490

NM 053195

AK031463

BC028556

NM_028724

NM 026425

AK079038

NM_025820

NM_175280

NM 016889

NM_001033348

AK020875

AK045105

AK035937

AK016684

SNPs SNP Density

$\begin{array}{ll}3 & 0.07 \\ 0 & 0.00\end{array}$

$0 \quad 0.00$

$0 \quad 0.00$

$1 \quad 0.10$

$18 \quad 0.36$

$1440 \quad 2.09$

$81 \quad 1.76$

0.00
$106 \quad 0.39$

$1 \quad 0.08$

$105 \quad 0.34$

$0 \quad 0.00$
1

0.07

0.00

0.03
0.06

0.03

0.03

0.06

1.23

2.34

0.00

3.05

3.23

40.00

6.80
4.42

3.58

3.43

3.90

1.88

13.55

4.48

0.00

0.56
7.79

3.17

3.65
5.41

4.42

246

3.33

2.32

6.26

0.00

2.79

3.24

1.75

11.76
3.11

3.11
3.78

4.51

3.69

5.06

3.37

4.47

3.84
3.17

1.33

3.71

3.22

4.37

0.02

0.00

0.00
0.00

0.03

0.00

0.03

0.00
0.00

0.00 
Table B-2. (Continued).

Position on Chr $2(\mathrm{Mb})$ Gene symbol Gene description

\begin{tabular}{|c|c|c|c|c|c|}
\hline Position on Chr 2 (Mb) & Gene symbol & Gene description & NM ID & SNPs & SNP Density \\
\hline 146.6816 & $\mathrm{Gm} 114$ & gene model 114, (NCBI) & NM_001033298 & 1 & 0.01 \\
\hline 146.8388 & Xrn2 & 5'-3' exoribonuclease 2 & NM_011917 & 0 & 0.00 \\
\hline 146.9096 & Nkx2-4 & NK2 transcription factor related, locus 4 (Drosophila) & NM_023504 & 0 & 0.00 \\
\hline 147.0089 & Nkx2-2 & NK2 transcription factor related, locus 2 (Drosophila) & NM_001077632 & 0 & 0.00 \\
\hline 147.0132 & 6430503K07Rik & RIKEN cDNA 6430503 K07 gene & AK0̈20097 & 0 & 0.00 \\
\hline 147.1873 & Al646519 & expressed sequence Al646519 & AB080658 & 0 & 0.00 \\
\hline 147.1907 & Pax1 & paired box gene 1 & NM_008780 & 0 & 0.00 \\
\hline 147.5364 & A530006G24Rik & RIKEN cDNA A530006G24 gene & AK0̄79921 & 65 & 9.37 \\
\hline 147.7705 & 9030622022Rik & RIKEN cDNA 9030622022 gene & AK018565 & 157 & 5.00 \\
\hline 147.8686 & Foxa2 & forkhead box $\mathrm{A} 2$ & NM_010446 & 4 & 0.98 \\
\hline 148.1062 & 2310021N16Rik & RIKEN cDNA 2310021N16 gene & AK009452 & 0 & 0.00 \\
\hline 148.2211 & Sstr4 & somatostatin receptor 4 & NM_009219 & 5 & 3.60 \\
\hline 148.2302 & Thbd & thrombomodulin & NM_009378 & 13 & 3.50 \\
\hline 148.2546 & 4833411/10Rik & RIKEN cDNA $4833411 \mid 10$ gene & AK0̄14679 & 2 & 1.28 \\
\hline 148.2624 & $\mathrm{Cd} 93$ & CD93 antigen & NM_010740 & 0 & 0.00 \\
\hline 148.4847 & Cst12 & cystatin 12 & 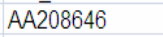 & 0 & 0.00 \\
\hline 148.4984 & Nxt1 & NTF2-related export protein 1 & NM_001110159 & 10 & 2.93 \\
\hline 148.5069 & Gzf1 & GDNF-inducible zinc finger protein 1 & NM_028986 & 26 & 2.20 \\
\hline 148.5204 & $\mathrm{Napb}$ & $\mathrm{N}$-ethylmaleimide sensitive fusion protein attachment protein beta & NM_019632 & 95 & 2.52 \\
\hline 148.5761 & Cstl1 & cystatin-like 1 & NM_177655 & 2 & 0.40 \\
\hline 148.5944 & Cst11 & cystatin 11 & NM_030059 & 0 & 0.00 \\
\hline 148.6077 & 8030411F24Rik & RIKEN cDNA $8030411 \mathrm{~F} 24$ gene & NM_030135 & 2 & 0.51 \\
\hline 148.6246 & Cst8 & cystatin 8 (cystatin-related epididymal spermatogenic) & NM_0099978 & 12 & 1.78 \\
\hline 148.6458 & Cst13 & cystatin 13 & NM_027024 & 40 & 3.88 \\
\hline 148.6609 & Cst9 & cystatin 9 & NM_0099979 & 7 & 1.95 \\
\hline 148.6724 & 9230104L09Rik & RIKEN cDNA 9230104L09 gene & NM_029960 & 1 & 0.24 \\
\hline 148.6907 & Gm1330 & gene model $1330,(\mathrm{NCBI})$ & BN0̄00358 & 0 & 0.00 \\
\hline 148.6975 & Cst3 & cystatin C & NM_009976 & 12 & 3.21 \\
\hline 149.0482 & LOC241715 & similar to chloride channel, nucleotide-sensitive, $1 \mathrm{~A}$ & XR_034975 & 2 & 51.28 \\
\hline 149.2310 & Cst10 & cystatin 10 (chondrocytes) & NM_021405 & 0 & 0.00 \\
\hline 149.6553 & C530025M09Rik & RIKEN cDNA C530025M09 gene & AK0̈35608 & 0 & 0.00 \\
\hline 149.7086 & LOC279056 & similar to XPMC2 prevents mitotic catastrophe 2 homolog & XR_034724 & 0 & 0.00 \\
\hline 149.9321 & 2010315B03Rik & RIKEN cDNA $2010315 \mathrm{~B} 03$ gene & AA1 155546 & 0 & 0.00 \\
\hline 149.9401 & Zfp120 & zinc finger protein 120 & NM 023266 & 2 & 0.09 \\
\hline
\end{tabular}


Table B-3. Genes and their SNPs in mapped locus on Chr $\mathrm{X}$ between 50-100Mb.

\begin{tabular}{|c|c|c|c|c|c|}
\hline Position on $\mathrm{Chr} X(\mathrm{Mb})$ & Gene symbol & Gene description & NM ID & SNPS & SNP Density \\
\hline 50.1444 & 6330534C20Rik & RIKEN cDNA 6330534C20 gene & NM_001034059 & 7 & 0.85 \\
\hline 50.2654 & Phf6 & PHD finger protein 6 & NM_027642 & 8 & 0.18 \\
\hline 50.3413 & Hprt1 & hypoxanthine guanine phosphoribosyl transferase 1 & NM_013556 & 8 & 0.24 \\
\hline 50.4063 & 9430052C07Rik & RIKEN cDNA $9430052 \mathrm{C} 07$ gene & AK020467 & 0 & 0.00 \\
\hline 50.4094 & C430049B03Rik & RIKEN cDNA C430049B03 gene & AK012483 & 0 & 0.00 \\
\hline 50.4232 & Plac1 & placental specific protein 1 & NM_019538 & 1 & 0.02 \\
\hline 50.5966 & 4632404H22Rik & RIKEN cDNA 4632404H22 gene & NM_030167 & 0 & 0.00 \\
\hline 50.6266 & 4930432H15Rik & RIKEN cDNA $4930432 \mathrm{H} 15$ gene & NM_028671 & 0 & 0.00 \\
\hline 50.6982 & Mospd1 & motile sperm domain containing 1 & NM_027409 & 0 & 0.00 \\
\hline 50.7881 & Etd & embryonic testis differentiation & NM_175147 & 0 & 0.00 \\
\hline 50.9111 & $\mathrm{Cxx} 1 \mathrm{C}$ & CAAX box 1 homolog $C$ (human) & NM_028375 & 0 & 0.00 \\
\hline 50.9457 & Cxx1a & CAAX box 1 homolog $A$ (human) & NM_024170 & 0 & 0.00 \\
\hline 50.9724 & $C \times x 1 b$ & CAAX box 1 homolog $B$ (human) & NM_001018063 & 0 & 0.00 \\
\hline 50.9897 & 4933416l08Rik & RIKEN cDNA 4933416108 gene & AK0̄16833 & 0 & 0.00 \\
\hline 50.9990 & AW822252 & expressed sequence AW822252 & AK045939 & 0 & 0.00 \\
\hline 51.0280 & 4930502E18Rik & RIKEN cDNA 4930502 E18 gene & NM_029142 & 0 & 0.00 \\
\hline 51.0461 & 1700013H16Rik & RIKEN cDNA $1700013 \mathrm{H} 16$ gene & AK0̄05953 & 0 & 0.00 \\
\hline 51.0759 & Zfp36l3 & zinc finger protein $36, \mathrm{C} 3 \mathrm{H}$ type-like 3 & NM_001009549 & 0 & 0.00 \\
\hline 51.0869 & XIr & X-linked lymphocyte-regulated complex & NM_0111725 & 0 & 0.00 \\
\hline 51.2726 & LOC100040271 & hypothetical protein LOC100040271 & AK 142406 & 0 & 0.00 \\
\hline 51.6523 & 4930527E24Rik & RIKEN cDNA 4930527E24 gene & AK015913 & 2 & 0.01 \\
\hline 52.6671 & 1600025M17Rik & RIKEN cDNA $1600025 \mathrm{M} 17$ gene & AA693115 & 0 & 0.00 \\
\hline 52.7250 & 6330419J24Rik & RIKEN cDNA 6330419J24 gene & Al846914 & 0 & 0.00 \\
\hline 53.3897 & 3830403N18Rik & RIKEN cDNA 3830403N18 gene & NM_027510 & 0 & 0.00 \\
\hline 53.4430 & Gm773 & gene model $773,(\mathrm{NCBI})$ & NM_001033423 & 3 & 0.13 \\
\hline 53.5996 & Zfp449 & zinc finger protein 449 & NM_030139 & 5 & 0.26 \\
\hline 53.6344 & 6530403M18Rik & RIKEN cDNA 6530403M18 gene & AK0 18320 & 0 & 0.00 \\
\hline 53.7081 & $\mathrm{Ddx} 26 \mathrm{~b}$ & DEAD/H (Asp-Glu-Ala-Asp/His) box polypeptide 26B & NM_172779 & 13 & 0.25 \\
\hline 53.7971 & Gm648 & gene model $648,(\mathrm{NCBI})$ & NM_001033372 & 0 & 0.00 \\
\hline 53.8099 & Gm364 & gene model 364 , (NCBI) & XM_141763 & 0 & 0.00 \\
\hline 53.8387 & Tmem32 & transmembrane protein 32 & NM_-146234 & 1 & 0.08 \\
\hline 53.8630 & SIc9a6 & solute carrier family 9 (sodium/hydrogen exchanger), isoform 6 & NM_172780 & 12 & 0.23 \\
\hline 53.9850 & Fhl1 & four and a half LIM domains 1 & NM_001077361 & 5 & 0.08 \\
\hline 54.0511 & Mtap7d3 & MAP7 domain containing 3 & NM_177293 & 3 & 0.12 \\
\hline 54.2166 & Gpr112 & G protein-coupled receptor 112 & NM_001110790 & 18 & 1.06 \\
\hline 54.2963 & Brs3 & bombesin-like receptor 3 & NM_009766 & 3 & 0.53 \\
\hline 54.3068 & Htatsf1 & HIV TAT specific factor 1 & NM_028242 & 2 & 0.15 \\
\hline 54.3413 & Vgll1 & vestigial like 1 homolog (Drosophila) & NM_133251 & 8 & 0.46 \\
\hline 54.4653 & Cd40lg & CD40 ligand & NM_011616 & 25 & 2.10 \\
\hline 54.4847 & Arhgef6 & $\mathrm{Rac} / \mathrm{Cdc} 42$ guanine nucleotide exchange factor (GEF) 6 & NM_152801 & 70 & 0.65 \\
\hline 54.6316 & 4732460l02Rik & RIKEN cDNA 4732460102 gene & AK0̄28836 & 0 & 0.00 \\
\hline 54.6395 & Rbmx & RNA binding motif protein, $\mathrm{X}$ chromosome & NM_011252 & 1 & 0.15 \\
\hline 54.7498 & Gpr101 & G protein-coupled receptor 101 & NM_001033360 & 0 & 0.00 \\
\hline 55.2152 & AY339874 & cDNA sequence AY339874 & AY $\overline{3} 39874$ & 0 & 0.00 \\
\hline 55.2838 & Zic3 & zinc finger protein of the cerebellum 3 & NM_009575 & 1 & 0.17 \\
\hline 56.1646 & 4930550L24Rik & RIKEN cDNA 4930550L24 gene & NM_023774 & 0 & 0.00 \\
\hline 56.3153 & Fgf13 & fibroblast growth factor 13 & NM_010200 & 2 & 0.03 \\
\hline 56.3458 & 9430082L08Rik & RIKEN cDNA 9430082L08 gene & AK0̄20501 & 0 & 0.00 \\
\hline 56.8093 & Gm715 & gene model $715,(\mathrm{NCBI})$ & XM_205232 & 0 & 0.00 \\
\hline 56.8377 & EG245436 & predicted gene, EG245436 & XM_891715 & 0 & 0.00 \\
\hline 57.2526 & F9 & coagulation factor IX & NM_007979 & 1 & 0.03 \\
\hline 57.3091 & Mcf2 & mcf. 2 transforming sequence & NM_133197 & 21 & 0.23 \\
\hline 57.3954 & C230004F18Rik & RIKEN cDNA C230004F18 gene & Al85̄1532 & 0 & 0.00 \\
\hline 57.4540 & C030023E24Rik & RIKEN cDNA C030023E24 gene & Al853519 & 0 & 0.00 \\
\hline 57.4765 & Atp11C & Atpase, class Vl, type $11 \mathrm{C}$ & NM_001001798 & 2 & 0.01 \\
\hline 57.5527 & EG245440 & predicted gene, EG245440 & XM_001473465 & 0 & 0.00 \\
\hline 58.1445 & Sox3 & SRY-box containing gene 3 & NM_009237 & 0 & 0.00 \\
\hline 58.3696 & LOC627159 & similar to Steroid hormone receptor ERR2 (Estrogen-related receptor, beta) & AK $\overline{1} 58351$ & 0 & 0.00 \\
\hline 58.9628 & Ldoc1 & leucine zipper, down-regulated in cancer 1 & NM_001018087 & 0 & 0.00 \\
\hline 59.5403 & 4933402E13Rik & RIKEN cDNA $4933402 E 13$ gene & AK016614 & 0 & 0.00 \\
\hline 59.7212 & 4931400007Rik & RIKEN cDNA 4931400007 gene & AK016421 & 0 & 0.00 \\
\hline 59.7637 & 1700019B21Rik & RIKEN cDNA $1700019 B 21$ gene & AK006108 & 0 & 0.00 \\
\hline 61.3096 & Ctag2 & cancer/testis antigen 2 & Al503560 & 0 & 0.00 \\
\hline 61.4046 & EG627470 & predicted gene, EG627470 & $\mathrm{BC} 048649$ & 0 & 0.00 \\
\hline 61.4267 & 3830417A13Rik & RIKEN cDNA 3830417 A13 gene & NM_027512 & 2 & 0.38 \\
\hline 61.5226 & Slitrk4 & SLIT and NTRK-like family, member 4 & NM_178740 & 1 & 0.13 \\
\hline 63.5565 & 4930447F04Rik & RIKEN cDNA 4930447F04 gene & AK $\overline{0} 15402$ & 0 & 0.00 \\
\hline 63.9025 & Slitrk2 & SLIT and NTRK-like family, member 2 & NM_198863 & 0 & 0.00 \\
\hline 63.9515 & Gm1140 & gene model 1140, (NCBI) & XM_001474142 & 0 & 0.00 \\
\hline 64.7114 & 1700036009Rik & RIKEN cDNA 1700036009 gene & AK006615 & 0 & 0.00 \\
\hline 65.1730 & 4933436l01Rik & RIKEN cDNA 4933436101 gene & NM_025763 & 0 & 0.00 \\
\hline 65.9317 & Fmr1 & fragile $\mathrm{X}$ mental retardation syndrome 1 homolog & NM_008031 & 48 & 1.22 \\
\hline 66.0151 & Fmr1nb & fragile $X$ mental retardation 1 neighbor & NM_174993 & 82 & 1.93 \\
\hline 66.1455 & EG627927 & predicted gene, EG627927 & NM_001098842 & 0 & 0.00 \\
\hline 66.6135 & Aff2 & AF4/FMR2 familv. member 2 & NM 008032 & 166 & 0.33 \\
\hline
\end{tabular}


Table B-3. (Continued).

\begin{tabular}{|c|c|c|c|c|c|}
\hline Position on $\mathrm{Chr}$ X (Mb) & Gene symbol & Gene description & NM ID & SNPS & SNP Density \\
\hline 66.6624 & 2610007B07Rik & RIKEN cDNA 2610007B07 gene & AK011331 & 0 & 0.00 \\
\hline 66.8250 & Hsfy2-ps & heat shock transcription factor, $Y$ linked 2 pseudogene & XM_290025 & 0 & 0.00 \\
\hline 67.1824 & 1700111N16Rik & RIKEN cDNA 1700111 N16 gene & AK007172 & 208 & 0.97 \\
\hline 67.1985 & 1700020N15Rik & RIKEN cDNA 1700020 N15 gene & AK006180 & 0 & 0.00 \\
\hline 67.5962 & Ids & iduronate 2-sulfatase & NM_001038990 & 2 & 0.09 \\
\hline 67.6391 & 1110012L19Rik & RIKEN cDNA 1110012L19 gene & NM_026787 & 1 & 0.29 \\
\hline 67.6471 & 4930567H17Rik & RIKEN cDNA 4930567H17 gene & NM_001033807 & 0 & 0.00 \\
\hline 67.7132 & BC023829 & cDNA sequence $\mathrm{BC} 023829$ & NM_001033328 & 24 & 1.41 \\
\hline 67.7987 & LOC628053 & hypothetical LOC628053 & NM_001081476 & 0 & 0.00 \\
\hline 68.2417 & Fate1 & fetal and adult testis expressed 1 & NR_003243 & 0 & 0.00 \\
\hline 68.3034 & Mamld1 & mastermind-like domain containing 1 & NM_001081354 & 36 & 0.34 \\
\hline 68.4683 & Mtm1 & X-linked myotubular myopathy gene 1 & NM_019926 & 102 & 1.02 \\
\hline 68.6179 & Mtmr1 & myotubularin related protein 1 & NM_016985 & 3 & 0.06 \\
\hline 68.6732 & Cd9912 & Cd99 antigen-like 2 & $\mathrm{NM}_{-} 138309$ & 1 & 0.01 \\
\hline 68.8092 & Hmgb3 & high mobility group box 3 & NM_008253 & 0 & 0.00 \\
\hline 68.9169 & Gpr50 & G-protein-coupled receptor 50 & NM_010340 & 0 & 0.00 \\
\hline 69.0621 & 2610030H06Rik & RIKEN cDNA $2610030 \mathrm{H} 06$ gene & NM_001081356 & 1 & 0.13 \\
\hline 69.1778 & Gm1141 & gene model $1141,(\mathrm{NCBI})$ & AK133067 & 8 & 0.95 \\
\hline 69.2084 & Prrg3 & proline rich Gla (G-carboxyglutamic acid) 3 (transmembrane) & NM_001081135 & 26 & 2.68 \\
\hline 69.2372 & Cnga2 & cyclic nucleotide gated channel alpha 2 & NM_007724 & 38 & 2.07 \\
\hline 69.4674 & Magea4 & melanoma antigen, family $A, 4$ & NM_020280 & 0 & 0.00 \\
\hline 69.5028 & Gabre & gamma-aminobutyric acid (GABA-A) receptor, subunit epsilon & NM_017369 & 8 & 0.46 \\
\hline 69.6272 & Magea10 & melanoma antigen family $A, 10$ & NM_001085506 & 0 & 0.00 \\
\hline 69.6792 & Gabra3 & gamma-aminobutyric acid (GABA-A) receptor, subunit alpha 3 & NM_008067 & 31 & 0.14 \\
\hline 70.0708 & Gabrq & gamma-aminobutyric acid (GABA-A) receptor, subunit theta & NM_020488 & 0 & 0.00 \\
\hline 70.1147 & Magea9 & melanoma antigen, family A, 9 & NM_181855 & 0 & 0.00 \\
\hline 70.1589 & Cetn2 & centrin 2 & NM_019405 & 0 & 0.00 \\
\hline 70.1639 & Nsdhl & NAD(P) dependent steroid dehydrogenase-like & NM_010941 & 6 & 0.15 \\
\hline 70.2327 & Zfp185 & zinc finger protein 185 & NM_001109043 & 3 & 0.07 \\
\hline 70.2793 & Pnma5 & paraneoplastic antigen family 5 & NM_001100461 & 0 & 0.00 \\
\hline 70.3101 & Pnma3 & paraneoplastic antigen MA3 & NM_153169 & 0 & 0.00 \\
\hline 70.3197 & Xlr4a & X-linked lymphocyte-regulated $4 \mathrm{~A}$ & NM_001081642 & 0 & 0.00 \\
\hline 70.3316 & Xlr3a & $\mathrm{X}$-linked lymphocyte-regulated $3 \mathrm{~A}$ & NM_001110784 & 3 & 0.28 \\
\hline 70.3530 & Xlr5a & X-linked lymphocyte-regulated $5 \mathrm{~A}$ & NM_001045539 & 11 & 1.09 \\
\hline 70.3826 & DXBay18 & DNA segment, $\mathrm{Chr} \mathrm{X}$, Baylor 18 & NM_001025384 & 19 & 1.55 \\
\hline 70.3942 & Xlr5b & X-linked lymphocyte-regulated $5 \mathrm{~B}$ & NM_001111293 & 21 & 2.20 \\
\hline 70.4141 & Tex28 & testis expressed 28 & XM_358216 & 0 & 0.00 \\
\hline 70.4206 & 4930408F14Rik & RIKEN cDNA $4930408 \mathrm{~F} 14$ gene & AK0 15110 & 0 & 0.00 \\
\hline 70.4375 & XIr3b & $\mathrm{X}$-linked lymphocyte-regulated $3 \mathrm{~B}$ & NM_001081643 & 9 & 0.84 \\
\hline 70.4597 & XIr $4 b$ & $\mathrm{X}$-linked lymphocyte-regulated $4 \mathrm{~B}$ & NM_021365 & 13 & 1.61 \\
\hline 70.4736 & F8a & factor 8-associated gene $A$ & NM_007978 & 4 & 1.61 \\
\hline 70.4794 & $\mathrm{X} \mid \mathrm{r} 4 \mathrm{c}$ & $\mathrm{X}$-linked lymphocyte-regulated $4 \mathrm{C}$ & NM_183094 & 10 & 1.10 \\
\hline 70.4999 & $\mathrm{XIr} 3 \mathrm{c}$ & $\mathrm{X}$-linked lymphocyte-regulated $3 \mathrm{C}$ & NM_011727 & 8 & 0.74 \\
\hline 70.5252 & Snora70 & small nucleolar RNA, H/ACA box 70 & NR_002899 & 0 & 0.00 \\
\hline 70.5305 & Xlr5c & $\mathrm{X}$-linked lymphocyte-regulated $5 \mathrm{C}$ & NM_031493 & 3 & 0.56 \\
\hline 70.5880 & Zfp275 & zinc finger protein 275 & NM_031494 & 1 & 0.06 \\
\hline 70.6564 & Zfp92 & zinc finger protein 92 & NM_009566 & 0 & 0.00 \\
\hline 70.6790 & Trex2 & three prime repair exonuclease 2 & NM_011907 & 0 & 0.00 \\
\hline 70.6827 & Uchl5ip & UCHL 5 interacting protein & NM_028633 & 2 & 0.09 \\
\hline 70.7290 & Bgn & biglycan & NM 007542 & 10 & 0.81 \\
\hline 70.7484 & Atp2b3 & ATPase, $\mathrm{Ca}++$ transporting, plasma membrane 3 & NM_177236 & 4 & 0.06 \\
\hline 70.8204 & EG245472 & predicted gene, EG245472 & XM_141845 & 0 & 0.00 \\
\hline 70.8848 & Dusp9 & dual specificity phosphatase 9 & NM_029352 & 3 & 0.74 \\
\hline 70.9013 & Pnck & pregnancy upregulated non-ubiquitously expressed CaM kinase & NM_012040 & 0 & 0.00 \\
\hline 70.9185 & Slc6a8 & solute carrier family 6 (neurotransmitter transporter, creatine), member 8 & NM_133987 & 0 & 0.00 \\
\hline 70.9315 & Bcap31 & B-cell receptor-associated protein 31 & NM_012060 & 10 & 0.33 \\
\hline 70.9619 & Abcd1 & ATP-binding cassette, sub-family D (ALD), member 1 & NM_007435 & 3 & 0.14 \\
\hline 71.0024 & Plxnb3 & plexin B3 & NM_019587 & 0 & 0.00 \\
\hline 71.0198 & Srpk3 & serine/arginine-rich protein specific kinase 3 & NM_019684 & 2 & 0.44 \\
\hline 71.0243 & $\operatorname{ldh} 3 \mathrm{~g}$ & isocitrate dehydrogenase $3(\mathrm{NAD}+$ ), gamma & NM_008323 & 0 & 0.00 \\
\hline 71.0324 & Ssr4 & signal sequence receptor, delta & NM 009279 & 0 & 0.00 \\
\hline 71.0387 & Pdzd4 & PDZ domain containing 4 & NM_001029868 & 7 & 0.22 \\
\hline 71.0991 & L1cam & L1 cell adhesion molecule & NM_008478 & 12 & 0.44 \\
\hline 71.1374 & Avpr2 & arginine vasopressin receptor 2 & NM_019404 & 0 & 0.00 \\
\hline 71.1397 & Arhgap4 & Rho GTPase activating protein 4 & NM_138630 & 1 & 0.06 \\
\hline 71.1622 & Ard1 & $\mathrm{N}$-acetyltransferase ARD1 homolog (S. cerevisiae) & NM_019870 & 0 & 0.00 \\
\hline 71.1675 & Renbp & renin binding protein & NM_023132 & 0 & 0.00 \\
\hline 71.1881 & Hcfc1 & host cell factor $\mathrm{C} 1$ & NM 008224 & 6 & 0.26 \\
\hline 71.2593 & Irak1 & interleukin-1 receptor-associated kinase 1 & NM_008363 & 0 & 0.00 \\
\hline 71.2722 & Mecp2 & methyl CpG binding protein 2 & NM_001081979 & 1 & 0.02 \\
\hline 71.3728 & Opn1mw & opsin 1 (cone pigments), medium-wave-sensitive (color blindness, deutan) & NM_008106 & 4 & 0.17 \\
\hline 71.4226 & Tkt11 & transketolase-like 1 & NM_031379 & 1 & 0.03 \\
\hline 71.4688 & Flna & filamin, alpha & NM_010227 & 3 & 0.13 \\
\hline 71.5002 & Emd & emerin & NM_007927 & 0 & 0.00 \\
\hline 71.5162 & Rpl10 & ribosomal protein 10 & NM 052835 & 0 & 0.00 \\
\hline
\end{tabular}


Table B-3. (Continued).

\begin{tabular}{|c|c|c|c|c|c|}
\hline Position on $\mathrm{Chr} \mathrm{X}(\mathrm{Mb})$ & Gene symbol & Gene description & NM ID & SNPS & SNP Density \\
\hline 71.5186 & Dnase1/1 & deoxyribonuclease 1-like 1 & NM_027109 & 0 & 0.00 \\
\hline 71.5281 & Taz & tafazzin & NM_181516 & 0 & 0.00 \\
\hline 71.5364 & B230340J04Rik & RIKEN cDNA B230340J04 gene & AK032075 & 0 & 0.00 \\
\hline 71.5425 & Atp6ap1 & ATPase, $\mathrm{H}+$ transporting, lysosomal accessory protein 1 & NM_018794 & 1 & 0.13 \\
\hline 71.5506 & Gdi1 & guanosine diphosphate (GDP) dissociation inhibitor 1 & NM_010273 & 0 & 0.00 \\
\hline 71.5584 & DOHXS9928E & DNA segment, human DXS9928E & NM_-138607 & 0 & 0.00 \\
\hline 71.5744 & Plxna3 & plexin A3 & NM_008883 & 2 & 0.13 \\
\hline 71.5975 & Lage3 & L antigen family, member 3 & NM_025410 & 0 & 0.00 \\
\hline 71.6127 & Ubl4 & ubiquitin-like 4 & NM_145405 & 0 & 0.00 \\
\hline 71.6146 & Slc10a3 & solute carrier family 10 (sodium/bile acid cotransporter family), member 3 & NM_145406 & 0 & 0.00 \\
\hline 71.6301 & 1810037C20Rik & RIKEN cDNA $1810037 \mathrm{C} 20$ gene & NM_025473 & 1 & 0.12 \\
\hline 71.6548 & G6pdx & glucose-6-phosphate dehydrogenase $\mathrm{X}$-linked & NM_008062 & 1 & 0.05 \\
\hline 71.6700 & lkbkg & inhibitor of kappaB kinase gamma & NM_010547 & 1 & 0.04 \\
\hline 71.7256 & EG628456 & predicted gene, EG628456 & NM_001099305 & 0 & 0.00 \\
\hline 71.8397 & Olfr1325 & olfactory receptor 1325 & NM_146398 & 0 & 0.00 \\
\hline 71.8845 & EG434797 & predicted gene, EG434797 & NM_001099302 & 0 & 0.00 \\
\hline 71.9863 & EG628518 & predicted gene, EG628518 & NM_001099306 & 0 & 0.00 \\
\hline 72.0825 & EG546325 & predicted gene, EG546325 & NM_001081670 & 0 & 0.00 \\
\hline 72.2339 & Gab3 & growth factor receptor bound protein 2-associated protein 3 & $\mathrm{NM}_{-} 153073$ & 59 & 0.61 \\
\hline 72.3413 & Dkc1 & dyskeratosis congenita 1 , dyskerin homolog (human) & NM_001030307 & 19 & 1.37 \\
\hline 72.3551 & Mpp1 & membrane protein, palmitoylated & NM_008621 & 23 & 1.08 \\
\hline 72.3914 & 4930428E23Rik & RIKEN cDNA 4930428E23 gene & AK132828 & 21 & 1.19 \\
\hline 72.4181 & F8 & coagulation factor VIII & NM_007977 & 312 & 1.50 \\
\hline 72.6278 & Fundc2 & FUN14 domain containing 2 & NM_026126 & 10 & 0.73 \\
\hline 72.6502 & Mtcp1 & mature T-cell proliferation 1 & NM_001039373 & 15 & 1.28 \\
\hline 72.6620 & Brcc3 & BRCA1/BRCA2-containing complex, subunit 3 & NM_145956 & 39 & 1.05 \\
\hline 72.7596 & Vbp1 & von Hippel-Lindau binding protein 1 & NM_011692 & 27 & 1.31 \\
\hline 72.8174 & Rab39b & RAB39B, member RAS oncogene family & $\mathrm{NM}_{-} 175122$ & 15 & 2.44 \\
\hline 72.9717 & 4933407K13Rik & RIKEN cDNA 4933407K13 gene & AK016728 & 60 & 2.05 \\
\hline 73.0310 & PIs3 & plastin 3 (T-isoform) & NM_145629 & 87 & 0.98 \\
\hline 73.5096 & EG238829 & predicted gene, EG238829 & NM_001013760 & 0 & 0.00 \\
\hline 73.5927 & Magea7 & melanoma antigen, family A, 7 & $\mathrm{BC} \overline{1} 07020$ & 0 & 0.00 \\
\hline 73.6387 & 4930428D18Rik & RIKEN cDNA 4930428D18 gene & NM_001033799 & 0 & 0.00 \\
\hline 73.8279 & 4930468A15Rik & RIKEN cDNA 4930468A15 gene & AK015525 & 6 & 0.30 \\
\hline 74.4969 & LOC236874 & similar to odorant binding protein la & XM_135951 & 0 & 0.00 \\
\hline 74.5877 & 5830424 K16Rik & RIKEN cDNA $5830424 K 16$ gene & AK017945 & 0 & 0.00 \\
\hline 74.7566 & Tbl1x & transducin (beta)-like $1 \mathrm{X}$-linked & NM_020601 & 14 & 0.09 \\
\hline 75.0074 & Prkx & protein kinase, $\mathrm{X}$-linked & NM_016979 & 12 & 0.35 \\
\hline 75.0832 & Pbsn & probasin & NM_017471 & 2 & 0.13 \\
\hline 75.1101 & OTTMUSG00000017 & predicted gene, OTTMUSG00000017677 & NM_001025383 & 0 & 0.00 \\
\hline 75.1101 & OTTMUSG00000017 & predicted gene, OTTMUSG00000017407 & XM_621007 & 0 & 0.00 \\
\hline 75.1101 & OTTMUSG00000017 & predicted gene, OTTMUSG00000017866 & NM_001085544 & 0 & 0.00 \\
\hline 75.1101 & OTTMUSG00000017 & predicted gene, OTTMUSG00000017976 & XM_204404 & 0 & 0.00 \\
\hline 75.1101 & OTTMUSG00000017 & predicted gene, OTTMUSG00000017155 & NM_001085351 & 0 & 0.00 \\
\hline 75.1101 & OTTMUSG00000017 & predicted gene, OTTMUSG00000017728 & XM_972942 & 0 & 0.00 \\
\hline 75.1101 & OTTMUSG00000017 & predicted gene, OTTMUSG00000017608 & AY512909 & 0 & 0.00 \\
\hline 75.1101 & OTTMUSG00000017 & predicted gene, OTTMUSG00000017540 & AK170409 & 0 & 0.00 \\
\hline 75.2336 & 5430402E10Rik & RIKEN cDNA $5430402 E 10$ gene & NM_027768 & 0 & 0.00 \\
\hline 75.3308 & Obp1a & odorant binding protein la & NM_008754 & 0 & 0.00 \\
\hline 75.3708 & EG546335 & predicted gene, EG546335 & NM_001085534 & 0 & 0.00 \\
\hline 75.6150 & 4930480E11Rik & RIKEN cDNA $4930480 E 11$ gene & AK0̄15598 & 0 & 0.00 \\
\hline 75.7153 & Prrg1 & proline rich Gla (G-carboxyglutamic acid) 1 & AK008151 & 19 & 0.17 \\
\hline 75.8206 & B930042K01Rik & RIKEN cDNA B930042K01 gene & AK040303 & 0 & 0.00 \\
\hline 75.9831 & 4921509A18Rik & RIKEN cDNA 4921509A18 gene & AK014848 & 0 & 0.00 \\
\hline 76.3137 & LOC211705 & similar to prohibitin & XR_032681 & 0 & 0.00 \\
\hline 76.5759 & EG667736 & predicted gene, EG667736 & NM_001099310 & 59 & 2.14 \\
\hline 76.7279 & EG636104 & predicted gene, EG636104 & NM_001099307 & 57 & 1.64 \\
\hline 76.8686 & 2410003J06Rik & RIKEN cDNA $2410003 J 06$ gene & NM_001113734 & 10 & 0.81 \\
\hline 78.3160 & Tmem47 & transmembrane protein 47 & NM_138751 & 1 & 0.04 \\
\hline 78.6649 & 4930595M18Rik & RIKEN cDNA 4930595M18 gene & NM_173435 & 13 & 0.34 \\
\hline 80.1942 & Dmd & dystrophin, muscular dystrophy & NM_007868 & 1317 & 0.58 \\
\hline 80.7320 & Tsga8 & testis specific gene $A 8$ & $\mathrm{AB} 0-32764$ & 0 & 0.00 \\
\hline 81.5228 & 1600014K23Rik & RIKEN cDNA $1600014 \mathrm{~K} 23$ gene & Al324104 & 0 & 0.00 \\
\hline 82.1414 & 5430427019Rik & RIKEN cDNA 5430427019 gene & AA175893 & 0 & 0.00 \\
\hline 82.6512 & Gm41 & gene model $41,(\mathrm{NCBI})$ & XM_111935 & 0 & 0.00 \\
\hline 82.8194 & Map3k7ip3 & mitogen-activated protein kinase kinase kinase 7 interacting protein 3 & NM_025729 & 85 & 1.41 \\
\hline 82.9473 & Gyk & glycerol kinase & NM_008194 & 53 & 0.71 \\
\hline 82.9792 & E130114A11Rik & RIKEN cDNA E130114A11 gene & AK021388 & 5 & 5.36 \\
\hline 83.0720 & LOC100041562 & hypothetical protein LOC100041562 & AK035200 & 4 & 0.41 \\
\hline 83.2895 & 1700072E05Rik & RIKEN cDNA 1700072 E05 gene & NM_028554 & 0 & 0.00 \\
\hline 83.4371 & NrOb1 & nuclear receptor subfamily 0 , group B, member 1 & NM_007430 & 0 & 0.00 \\
\hline 83.4956 & CN716893 & expressed sequence CN716893 & NM_001033492 & 3 & 0.50 \\
\hline 83.9863 & 6330532G10Rik & RIKEN cDNA 6330532G10 gene & AK018225 & 0 & 0.00 \\
\hline 83.9926 & ||1rapl1 & interleukin 1 receptor accessory protein-like 1 & AK081272 & 39 & 0.03 \\
\hline 84.9261 & 2900005I04Rik & RIKEN cDNA 2900005104 gene & AK013482 & 0 & 0.00 \\
\hline
\end{tabular}


Table B-3. (Continued).

\begin{tabular}{|c|c|c|c|c|c|}
\hline Position on $\mathrm{Chr} \mathrm{X}(\mathrm{Mb})$ & Gene symbol & Gene description & NM ID & SNPS & SNP Density \\
\hline 86.1818 & 4930415L06Rik & RIKEN cDNA 4930415L06 gene & NR_003621 & 0 & 0.00 \\
\hline 86.4519 & Gm370 & gene model 370 , (NCBI) & XM_141921 & 0 & 0.00 \\
\hline 86.6492 & Pet2 & plasmacytoma expressed transcript 2 & NM_008821 & 0 & 0.00 \\
\hline 86.9976 & 4932429P05Rik & RIKEN cDNA 4932429P05 gene & NM_001085511 & 0 & 0.00 \\
\hline 87.7338 & EG278167 & predicted gene, EG278167 & XM_205261 & 0 & 0.00 \\
\hline 88.3464 & EG236893 & predicted gene, EG236893 & XM_141935 & 0 & 0.00 \\
\hline 88.5771 & Mageb1 & melanoma antigen, family $B, 1$ & NM_010759 & 0 & 0.00 \\
\hline 88.5772 & Mageb2 & melanoma antigen, family $B, 2$ & NM_031171 & 0 & 0.00 \\
\hline 88.8206 & 1700084M14Rik & RIKEN cDNA $1700084 \mathrm{M} 14$ gene & AK0006997 & 0 & 0.00 \\
\hline 88.8775 & Mageb5 & melanoma antigen, family B, 5 & AK006807 & 0 & 0.00 \\
\hline 89.1773 & EG278087 & predicted gene, EG278087 & NM_001083629 & 0 & 0.00 \\
\hline 89.3642 & Mageb18 & melanoma antigen family B, 18 & NM_173783 & 60 & 0.12 \\
\hline 89.7353 & EG546347 & predicted gene, EG546347 & NM_001034103 & 0 & 0.00 \\
\hline 90.2356 & EG245516 & predicted gene, EG245516 & XM_001472506 & 0 & 0.00 \\
\hline 90.4015 & $\mathrm{RP} 23-438 \mathrm{H} 3.2$ & hypothetical protein LOC69357 & NM_183280 & 0 & 0.00 \\
\hline 90.4016 & 1700003E24Rik & RIKEN cDNA $1700003 E 24$ gene & AK 005630 & 0 & 0.00 \\
\hline 90.5320 & Arx & aristaless related homeobox gene (Drosophila) & NM_007492 & 0 & 0.00 \\
\hline 90.5501 & Pola1 & polymerase (DNA directed), alpha 1 & NM_008892 & 5 & 0.02 \\
\hline 90.9002 & Pcyt1b & phosphate cytidylyltransferase 1 , choline, beta isoform & NM_177546 & 14 & 0.15 \\
\hline 91.0100 & Pdk3 & pyruvate dehydrogenase kinase, isoenzyme 3 & NM_145630 & 0 & 0.00 \\
\hline 91.2001 & Gm371 & gene model 371 , (NCBI) & XM_141955 & 0 & 0.00 \\
\hline 91.2140 & AU015836 & expressed sequence AU015836 & AK 145358 & 0 & 0.00 \\
\hline 91.3200 & Zfx & zinc finger protein $\mathrm{X}$-linked & NM_001044386 & 0 & 0.00 \\
\hline 91.4340 & Eif2s $3 x$ & eukaryotic translation initiation factor 2 , subunit 3 , structural gene X-linked & NM_012010 & 1 & 0.04 \\
\hline 91.4803 & Klhl15 & kelch-like 15 (Drosophila) & NM_001039059 & 0 & 0.00 \\
\hline 91.6004 & 4932442L08Rik & RIKEN cDNA 4932442L08 gene & AK016569 & 0 & 0.00 \\
\hline 91.6124 & Apoo & apolipoprotein $\mathrm{O}$ & NM_026673 & 0 & 0.00 \\
\hline 91.7808 & Maged1 & melanoma antigen, family $\mathrm{D}, 1$ & NM_019791 & 0 & 0.00 \\
\hline 91.8814 & Gspt2 & G1 to $S$ phase transition 2 & NM_008179 & 0 & 0.00 \\
\hline 91.9699 & $Z x d b$ & zinc finger, $X$-linked, duplicated $B$ & NM_001081473 & 0 & 0.00 \\
\hline 92.2178 & Spin4 & spindlin family, member 4 & NM_178753 & 1 & 0.24 \\
\hline 92.2443 & Arhgef9 & Cdc42 guanine nucleotide exchange factor (GEF) 9 & NM_001033329 & 1 & 0.01 \\
\hline 92.3544 & 5730407005Rik & RIKEN cDNA 5730407005 gene & AK017518 & 0 & 0.00 \\
\hline 92.6157 & 2810002009 Rik & RIKEN cDNA 2810002009 gene & NM_175179 & 0 & 0.00 \\
\hline 92.6655 & Asb12 & ankyrin repeat and SOCS box-containing protein 12 & NM_080858 & 0 & 0.00 \\
\hline 92.8345 & AK129302 & cDNA sequence AK129302 & NM_001003916 & 0 & 0.00 \\
\hline 92.9070 & Zc3h12b & zinc finger $\mathrm{CCCH}$-type containing $12 \mathrm{~B}$ & NM_001034907 & 2 & 0.01 \\
\hline 92.9279 & 1700010D01Rik & RIKEN CDNA 1700010D01 gene & NM_029590 & 0 & 0.00 \\
\hline 93.1307 & Las1l & LAS1-like (S. cerevisiae) & NM_152822 & 0 & 0.00 \\
\hline 93.2914 & Msn & moesin & NM_010833 & 2 & 0.03 \\
\hline 93.4353 & ENSMUSG00000065 & predicted gene, ENSMUSG00000065522 & AK 170206 & 1 & 0.27 \\
\hline 93.4353 & ENSMUSG00000065 & predicted gene, ENSMUSG00000065996 & AK086046 & 1 & 0.27 \\
\hline 93.4425 & Vsig4 & V-set and immunoglobulin domain containing 4 & NM_177789 & 0 & 0.00 \\
\hline 93.5022 & B230358A15Rik & RIKEN cDNA B230358A15 gene & NM_172931 & 1 & 0.02 \\
\hline 93.6508 & Heph & hephaestin & NM_010417 & 0 & 0.00 \\
\hline 93.9089 & Gpr165 & G protein-coupled receptor 165 & NM_029536 & 0 & 0.00 \\
\hline 94.1294 & 1700125M20Rik & RIKEN cDNA $1700125 \mathrm{M} 20$ gene & AK007284 & 0 & 0.00 \\
\hline 94.2679 & Pgr15l & G protein-coupled receptor 15 -like & NM_001033361 & 1 & 0.11 \\
\hline 94.5292 & Eda2r & ectodysplasin A2 isoform receptor & NM_175540 & 0 & 0.00 \\
\hline 95.3451 & $\mathrm{Ar}$ & androgen receptor & NM_013476 & 5 & 0.03 \\
\hline 95.7529 & Ophn1 & oligophrenin 1 & NM_052976 & 2 & 0.01 \\
\hline 96.1331 & Yipf6 & Yip1 domain family, member 6 & NM_207633 & 1 & 0.09 \\
\hline 96.2380 & Stard8 & START domain containing 8 & NM_199018 & 0 & 0.00 \\
\hline 96.3315 & Efnb1 & ephrin B1 & NM_010110 & 1 & 0.08 \\
\hline 96.6007 & OTTMUSG00000018 & predicted gene, OTTMUSG00000018981 & XM_001473979 & 0 & 0.00 \\
\hline 96.6007 & OTTMUSG00000018 & predicted gene, OTTMUSG00000018988 & XM_486794 & 0 & 0.00 \\
\hline 96.6007 & OTMUSG00000018 & predicted gene, OTTMUSG00000018964 & XM_982089 & 0 & 0.00 \\
\hline 96.6007 & OTTMUSG00000018 & predicted gene, OTTMUSG00000018973 & XM_982748 & 0 & 0.00 \\
\hline 96.6007 & OTTMUSG00000018 & predicted gene, OTTMUSG00000018874 & AK033508 & 0 & 0.00 \\
\hline 96.6007 & OTTMUSG00000018 & predicted gene, OTTMUSG00000018617 & AK045662 & 0 & 0.00 \\
\hline 96.6007 & OTTMUSG00000018 & predicted gene, OTTMUSG00000018077 & AK132930 & 0 & 0.00 \\
\hline 96.6007 & OTTMUSG00000018 & predicted gene, OTTMUSG00000018931 & AK147193 & 0 & 0.00 \\
\hline 96.6007 & OTTMUSG00000018 & predicted gene, OTTMUSG00000018066 & AK135730 & 0 & 0.00 \\
\hline 96.6007 & OTTMUSG00000018 & predicted gene, OTTMUSG00000018358 & AK076701 & 0 & 0.00 \\
\hline 96.6611 & Pja1 & praja1, RING-H2 motif containing & NM_001083110 & 0 & 0.00 \\
\hline 97.0164 & Tnen28 & transmembrane protein 28 & NM_001081283 & 0 & 0.00 \\
\hline 97.1709 & Eda & ectodysplasin-A & NM_010099 & 11 & 0.03 \\
\hline 97.4047 & D030036P13Rik & RIKEN cDNA D030036P13 gene & Al853652 & 0 & 0.00 \\
\hline 97.5976 & Dgat214 & diacylglycerol O-acyltransferase 2-like 4 & NM_177746 & 1 & 0.10 \\
\hline 97.6244 & Otud6a & OTU domain containing $6 \mathrm{~A}$ & BC153817 & 0 & 0.00 \\
\hline 97.6897 & Igbp1 & immunoglobulin (CD79A) binding protein 1 & NM_008784 & 0 & 0.00 \\
\hline 97.7202 & Dgat216 & diacylglycerol O-acyltransferase 2-like 6 & NM_001114084 & 0 & 0.00 \\
\hline 97.7676 & Dgat2|3 & diacylglycerol O-acyltransferase 2 -like 3 & NM_001081136 & 0 & 0.00 \\
\hline 97.7855 & P2ry 4 & pyrimidinergic receptor $\mathrm{P} 2 \mathrm{Y}, \mathrm{G}$-protein coupled, 4 & NM_020621 & 0 & 0.00 \\
\hline 97.8009 & Arr3 & arrestin 3 , retinal & NM 133205 & 0 & 0.00 \\
\hline
\end{tabular}


Table B-3. (Continued).

\begin{tabular}{|c|c|c|c|c|c|}
\hline Position on $\mathrm{Chr} \mathrm{X}(\mathrm{Mb})$ & Gene symbol & Gene description & NM ID & SNPS & SNP Density \\
\hline 97.8182 & Pdzd11 & PDZ domain containing 11 & NM_028303 & 0 & 0.00 \\
\hline 97.8214 & Kif4 & kinesin family member 4 & NM_008446 & 0 & 0.00 \\
\hline 97.9252 & Gdpd2 & glycerophosphodiester phosphodiesterase domain containing 2 & NM_023608 & 0 & 0.00 \\
\hline 97.9631 & Dlg3 & discs, large homolog 3 (Drosophila) & NM_016747 & 1 & 0.02 \\
\hline 97.9943 & EG212753 & predicted gene, EG212753 & XM_001478790 & 0 & 0.00 \\
\hline 98.0219 & Gm1717 & gene model $1717,(\mathrm{NCBI})$ & $\mathrm{Al} 4 \overline{4} 9690$ & 0 & 0.00 \\
\hline 98.0340 & Tex11 & testis expressed gene 11 & NM 031384 & 12 & 0.05 \\
\hline 98.2746 & Slc7a3 & solute carrier family 7 (cationic amino acid transporter, $y+$ system), member 3 & NM_007515 & 0 & 0.00 \\
\hline 98.2931 & Snx12 & sorting nexin 12 & NM_001110310 & 4 & 0.03 \\
\hline 98.2945 & 1110062M06Rik & RIKEN cDNA 1110062M06 gene & Al22̄5852 & 0 & 0.00 \\
\hline 98.3095 & LOC331476 & similar to SUMO-1-specific protease & XM_001474143 & 0 & 0.00 \\
\hline 98.3269 & E130102C15Rik & RIKEN cDNA E130102C15 gene & AK021367 & 0 & 0.00 \\
\hline 98.4499 & Foxo4 & forkhead box 04 & NM_018789 & 0 & 0.00 \\
\hline 98.4567 & Gm614 & gene model 614 , (NCBI) & NM_001033362 & 0 & 0.00 \\
\hline 98.4597 & $\| 2 \mathrm{rg}$ & interleukin 2 receptor, gamma chain & NM_013563 & 0 & 0.00 \\
\hline 98.4694 & Med12 & mediator of RNA polymerase II transcription, subunit 12 homolog (yeast) & NM_021521 & 0 & 0.00 \\
\hline 98.4945 & Nlgn 3 & neuroligin 3 & NM 172932 & 0 & 0.00 \\
\hline 98.5180 & 2900075N08Rik & RIKEN cDNA 2900075 N08 gene & AK0̄13791 & 0 & 0.00 \\
\hline 98.5727 & Gjb1 & gap junction protein, beta 1 & NM_008124 & 0 & 0.00 \\
\hline 98.5997 & Zmym3 & zinc finger, MYM-type 3 & NM_019831 & 0 & 0.00 \\
\hline 98.6251 & Nono & non-POU-domain-containing, octamer binding protein & NM_023144 & 5 & 0.26 \\
\hline 98.6444 & Itgb1bp2 & integrin beta 1 binding protein 2 & NM 013712 & 2 & 0.45 \\
\hline 98.7281 & Taf1 & TAF1 RNA polymerase II, TATA box binding protein (TBP)-associated factor & NM_001081008 & 5 & 0.07 \\
\hline 98.8354 & Ogt & O-linked $\mathrm{N}$-acetylglucosamine (GIcNAc) transferase & NM_139144 & 10 & 0.23 \\
\hline 98.8399 & 8430425A16Rik & RIKEN cDNA $8430425 \mathrm{~A} 16$ gene & AK $0 \overline{1} 18439$ & 0 & 0.00 \\
\hline 98.9269 & Cxcr3 & chemokine (C-X-C motif) receptor 3 & NM_009910 & 0 & 0.00 \\
\hline 98.9900 & 8030474K03Rik & RIKEN CDNA $8030474 K 03$ gene & AK033245 & 2 & 0.50 \\
\hline 99.0447 & EG621083 & predicted gene, EG621083 & AK142977 & 21 & 0.21 \\
\hline 99.2619 & Rgag4 & retrotransposon gag domain containing 4 & NM_183318 & 0 & 0.00 \\
\hline 99.2697 & Pabpc1/2a & poly (A) binding protein, cytoplasmic 1 -like $2 \mathrm{~A}$ & XM-141989 & 0 & 0.00 \\
\hline 99.2799 & $6430511 \mathrm{~F} 03$ & hypothetical protein $6430511 \mathrm{~F} 03$ & AK $\overline{0} 32250$ & 0 & 0.00 \\
\hline 99.3148 & Pin4 & protein (peptidyl-prolyl cis/trans isomerase) NIMA-interacting, 4 (parvulin) & NM_027181 & 1 & 0.12 \\
\hline 99.3383 & Ercc6l & excision repair cross-complementing rodent repair deficiency complementation group 6 - like & NM_-146235 & 3 & 0.21 \\
\hline 99.3803 & $\operatorname{Rps} 4 \mathrm{x}$ & ribosomal protein S4, X-linked & NM_009094 & 3 & 0.87 \\
\hline 99.4428 & Cited1 & $\mathrm{Cbp} / \mathrm{p} 300$-interacting transactivator with Glu/Asp-rich carboxy-terminal domain 1 & NM_007709 & 3 & 0.69 \\
\hline 99.4480 & LOC434825 & hypothetical gene supported by AK090266 & AK0̄90266 & 1 & 0.20 \\
\hline 99.4800 & Hdac8 & histone deacetylase 8 & NM_027382 & 36 & 0.16 \\
\hline 99.5106 & 4732468M13Rik & RIKEN cDNA 4732468M13 gene & AK0̈41832 & 0 & 0.00 \\
\hline 99.6663 & Xist & inactive $X$ specific transcripts & NR_001463 & 0 & 0.00 \\
\hline 99.6874 & Tsix & $X$ (inactive)-specific transcript, antisense & NR_002844 & 0 & 0.00 \\
\hline 99.7093 & Phka1 & phosphorylase kinase alpha 1 & NM_0008832 & 43 & 0.33 \\
\hline 99.9032 & Dmrtc1b & DMRT-like family $\mathrm{C} 1 \mathrm{~b}$ & NM_001039116 & 1 & 0.14 \\
\hline 99.9865 & EG382233 & predicted gene, EG382233 & $\mathrm{BC} 049655$ & 3 & 0.42 \\
\hline
\end{tabular}




\section{APPENDIX C. POLYMORPHISM ANALYSIS OF GENES AND TRANSCRIPTS IN THE MAPPED LOCI}

This appendix contains lists of single nucleotide polymorphism (SNPs) of genes and transcripts in the mapped quantitative trait loci (QTLs) associated with differential susceptibility to severe group A streptococcal (GAS) sepsis. The analysis was done on selected highly susceptible and highly resistant strains, namely, BXD77, 97, 100 and 75 as highly susceptible strains. In contrast, BXD69, 73, 48 and 87 are highly resistant strains, in addition, both parental strains B6 and D2 were included in this analysis. I performed the analysis on May 1, 2010 using mouse phenome database (MPD) employing the SNP wizard online tool at http://phenome.jax.org/SNP. The retrieved data was based on several databases including; national center for biotechnology information (NCBI) Mouse Build 37 (known as mm9), NCBI SNP database (dbSNP) build 128 and the joint project of the European Molecular Biology Laboratory (EMBL), European Bioinformatics Institute (EBI) and the Wellcome Trust Sanger Institute, (Ensembl) build 48. SNPs among BXD strains were based on Wellcome-CTC Mouse Strain SNP Genotype Set (Shifman et al., 2006).

There are three tables in this appendix:

- Table C-1. SNP analysis of genes in mapped locus on Chr 2 between 22-34 Mb.

- Table C-2. SNP analysis of genes in mapped locus on Chr 2 between 125-150 Mb.

- Table C-3. SNP analysis of genes in mapped locus on Chr X between 50-100 Mb.

The tables in this appendix contain the following abbreviations for SNP functional classes retrieved:

Cn Coding non-synonymous; SNP changes the amino acid/peptide,

Cs Coding synonymous; SNP does not change the amino acid/peptide,

UTR Untranslated region, SNP is in non-coding interval of mRNA, $\mathrm{S} \quad \mathrm{SNP}$ is in a splice-site; first two or last two bases of intron (few), I SNP is in an intron, except first 2 and last 2 bases (see above splice-site SNP), and L Locus region; not assigned to a gene; can be in region of gene but not in transcribed region; or may be in regulatory region. 
Table C-1. SNP analysis of genes in mapped locus on Chr 2 between 22-34 Mb.

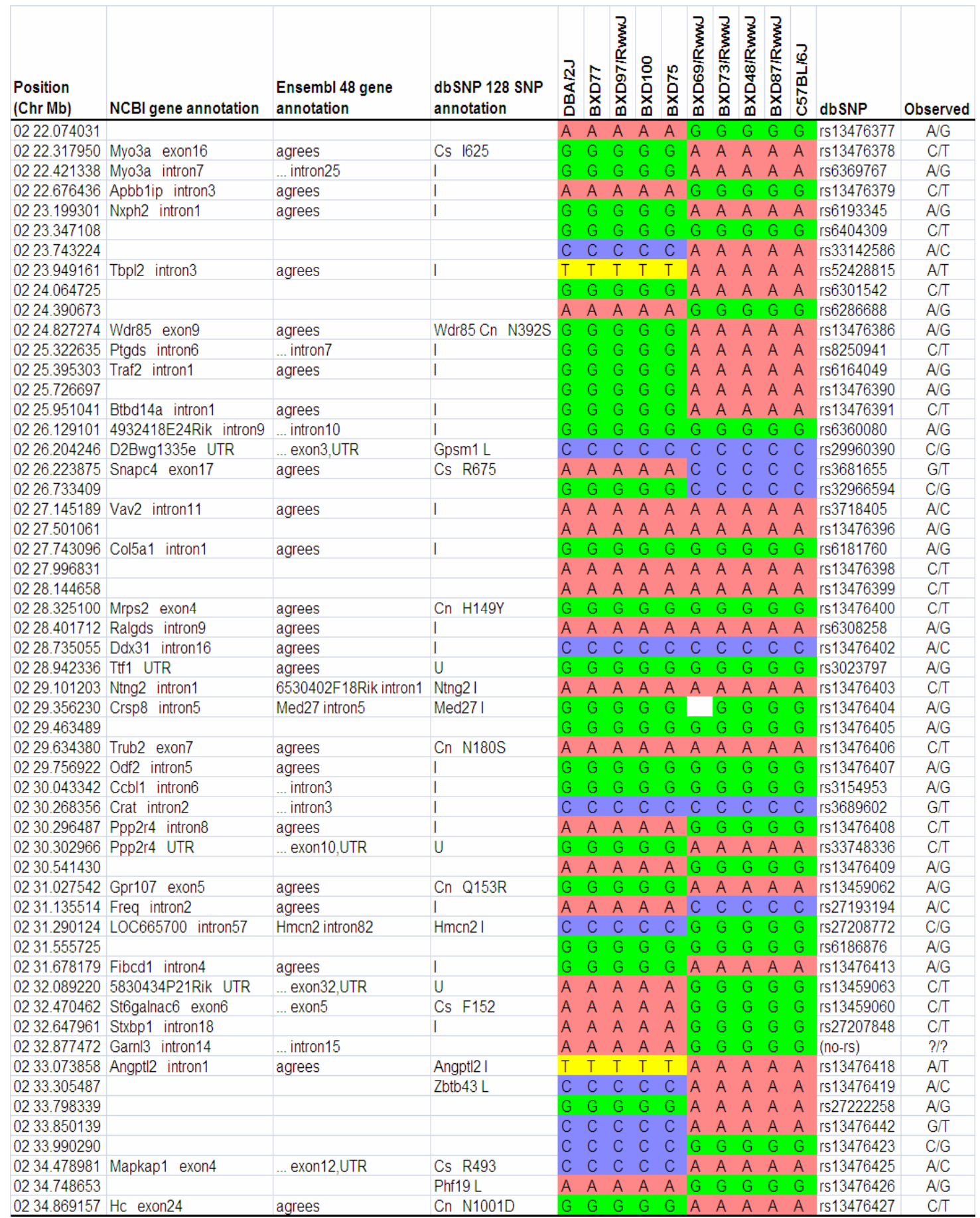


Table C-2. SNP analysis of genes in mapped locus on Chr 2 between 125-150 Mb.

\begin{tabular}{|c|c|c|c|c|c|c|c|c|c|c|c|c|c|c|c|}
\hline $\begin{array}{l}\text { Position } \\
\text { (Chr Mb) } \\
\end{array}$ & NCBI gene annotation & Ensembl 48 gene annotation & $\begin{array}{l}\mathrm{dbSNP} 128 \mathrm{SNP} \\
\text { annotation }\end{array}$ & 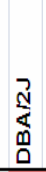 & $\begin{array}{l}\hat{N} \\
0 \\
0 \\
0\end{array}$ & 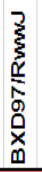 & $\begin{array}{l}\stackrel{\circ}{\circ} \\
\stackrel{x}{x} \\
0\end{array}$ & & 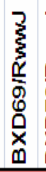 & 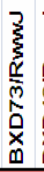 & 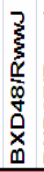 & 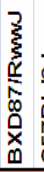 & 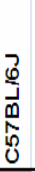 & $\mathrm{dbSNP}$ & Observed \\
\hline 02125.123509 & & & & $A$ & $A$ & $\bar{A}$ & $\bar{A}$ & $A$ & A & $\bar{A}$ & $\bar{A}$ & $\overline{A A}$ & $\bar{A}$ & rs6401493 & $\mathrm{C} / \mathrm{T}$ \\
\hline 02125.127623 & Fbn1 exon66 & agrees & Cn N2773S & A & $A$ & A & $A$ & A & A & A & A & A $A$ & A & rs 8251635 & $\mathrm{C} T$ \\
\hline 02125.304785 & Fbn1 intron3 & agrees & I & A & G & A & G & A & G & G & G & G & G & rs 6374387 & $\mathrm{C} / \mathrm{T}$ \\
\hline 02125.364688 & & & & G & G & G & G & G & G & G & G & G & G & rs3697020 & $\mathrm{C} / \mathrm{T}$ \\
\hline 02125.564678 & 3110001l20Rik UTR & ... exon18,UTR & U & A & C & A & C & A & C & C & C & C & C & rs 3725315 & $\mathrm{G} / \mathrm{T}$ \\
\hline 02125.704535 & Galk2 intron2 & agrees & I & C & A & C & A & C & A & A & A & A $A$ & A & rs 3708635 & $\mathrm{G} / \mathrm{T}$ \\
\hline 02125.866915 & Fgf7 intron2 & agrees & I & G & $A$ & G & A & $G$ & A & A & A & $A A$ & A & rs3667348 & $A / G$ \\
\hline 02126.005696 & & & & G & $A$ & G & $A$ & G & A & A & A & $A A$ & A & rs3704471 & $\mathrm{C} / \mathrm{T}$ \\
\hline 02126.259800 & & Atp8b4 intron6 & & A & G & A & G & A & G & G & G & G & G & rs6369977 & $A / G$ \\
\hline 02126.470820 & Gabpb1 intron7 & agrees & I & G & C & G & $\mathrm{C}$ & G & C & C & C & C & C & rs3720259 & $\mathrm{C} / \mathrm{G}$ \\
\hline 02126.557730 & Usp8 intron4 & ... intron5 & 1 & G & $\mathrm{C}$ & $G$ & C & G & C & C & C & C & C & rs 13476758 & $\mathrm{C} / \mathrm{G}$ \\
\hline 02126.929174 & & & Ncaph L & A & G & A & G & A & G & G & G & G & G & rs 4223448 & $\mathrm{C} \pi$ \\
\hline 02126.967277 & 1700041B20Rik exon2 & ... exon3,UTR & Cs G259 & G & A & G & A & G & A & A , & A & A & A & rs 13476760 & $\mathrm{C} / \mathrm{T}$ \\
\hline 02127.292727 & Kcnip3 intron2 & agrees & I & G & G & G & G & G & G & G & G & G & G & rs 13476761 & $\mathrm{C} / \mathrm{T}$ \\
\hline 02127.522306 & & & & A & G & A & G & A & G & G & G & G & G & rs 3680660 & $\mathrm{CT}$ \\
\hline 02127.787332 & Acoxl introng & agrees & I & C & G & $\mathrm{C}$ & G & C & G & G & G & G & G & rs 13476763 & $\mathrm{C} / \mathrm{G}$ \\
\hline 02127.840969 & Acoxl intron12 & agrees & I & A & G & A & G & $A$ & G & G & G & G & G & rs 6280404 & $\mathrm{C} / \mathrm{T}$ \\
\hline 02127.974055 & $\mathrm{Bc}|2| 11$ intron3 & ... intron4 & I & G & A & G & A & G & $\mathrm{G}$ & A & A & A & A & rs 13476764 & $\mathrm{C} / \mathrm{T}$ \\
\hline 02128.199227 & & OTTMUSG00000015351 intron 1 & LOC100043424I & G & $A$ & G & A & G & A & A & A & A & A & rs6411422 & $A / G$ \\
\hline 02128.300336 & & & & G & $A$ & G & A & G & A & A & A & A & A & rs13476765 & $\mathrm{C} / \mathrm{T}$ \\
\hline 02128.383738 & & & & G & $A$ & G & A & G & A & A & A & A & A & rs 6325084 & $\mathrm{C} / \mathrm{T}$ \\
\hline 02128.617919 & Mertk intron 16 & agrees & 1 & G & A & G & A & G & A & A & A & A & A & rs 13476766 & $A / G$ \\
\hline 02128.877858 & & & & G & $A$ & G & A & G & A & A & A & A & $A$ & rs 3671147 & $\mathrm{C} / \mathrm{T}$ \\
\hline 02128.959466 & Chchd5 UTR & ... exon4,UTR & U & G & A & G & A & G & A & A & A & A & A & rs 13459165 & $\mathrm{C} / \mathrm{T}$ \\
\hline 02128.959490 & Chchd5 UTR & ... exon4,UTR & U & $\mathrm{T}$ & A & $\mathrm{T}$ & A & $\mathrm{T}$ & A & A & A & A & A & rs13469412 & AT \\
\hline 02129.049210 & A730036l17Rik intron1 & agrees & I & G & A & G & A & G & A & A & A & A & A & rs 3662407 & $A / G$ \\
\hline 02129.089790 & LOC671466 intron2 & & I & G & G & G & G & G & G & G & G & G & G & rs6318992 & $A / G$ \\
\hline 02129.125489 & \|1a UTR & ... exon7,UTR & U & A & $\mathrm{T}$ & A & $\mathrm{T}$ & A & $\mathrm{T}$ & $\mathrm{T}$ & T & $\mathrm{T}$ & $\mathrm{T}$ & rs 3022902 & $\mathrm{ATT}$ \\
\hline 02129.383337 & & & & $\mathrm{~T}$ & $T$ & $T$ & $T$ & $T$ & $\mathrm{~T}$ & $\mathrm{~T}$ & T & $\mathrm{T}$ & $\mathrm{T}$ & rs 13476768 & AT \\
\hline 02129.457556 & Sirpa UTR & ... exon9,UTR & U & A & $A$ & $A$ & A & A & A & A & A & A & A & rs 27257479 & $\mathrm{G} / \mathrm{T}$ \\
\hline 02129.542808 & & & & A & $A$ & A & A & A & A & $A$ & A & A & A & rs6161193 & $\mathrm{A} / \mathrm{C}$ \\
\hline 02129.928492 & AU015228 UTR & & U & G & G & G & G & G & G & G & G & G & G & rs 4223491 & $A / G$ \\
\hline 02129.970551 & Tgm6 intron9 & agrees & I & A & $A$ & $A$ & $A$ & $A$ & A & A & $A$ & A & A & rs 3661596 & $\mathrm{C} \pi$ \\
\hline 02130.216766 & Cpxm1 exon14 & agrees & Cs G680 & G & G & G & G & G & G & G & G & G & G & rs 4223497 & $\mathrm{C} / \mathrm{T}$ \\
\hline 02130.367115 & Ptpra exon13 & ... exon14 & Cs Y497 & G & $G$ & $G$ & $G$ & G & G & G & G & G & G & rs 13459166 & $\mathrm{C} / \mathrm{T}$ \\
\hline 02130.507288 & Itpa UTR & ... exon8,UTR & U & A & $A$ & $A$ & $A$ & $A$ & A & $A$ & A & A & A & rs 13465151 & ATT \\
\hline 02130.534029 & 4930402H24Rik UTR & ... exon37,UTR & U & A & $A$ & $A$ & A & $A$ & A & A & A & A & $A$ & rs 4223500 & $A / G$ \\
\hline 02130.777824 & Atrn intron6 & agrees & I & A & $A$ & $A$ & A & $A$ & A & $A$ & A & A & A & rs 13476772 & $\mathrm{C} / \mathrm{T}$ \\
\hline 02130.839645 & Atrn intron6 & ... intron 25 & 1 & G & G & G & G & G & G & G & G & G & G & rs 3665528 & $A / G$ \\
\hline 02130.877524 & Adam33 intron19 & agrees & I & C & C & $\mathrm{C}$ & C & C & C & $\mathrm{C}$ & C & C & C & rs8264979 & $\mathrm{G} / \mathrm{T}$ \\
\hline 02130.881869 & Adam33 intron6 & agrees & I & A & G & A & G & A & G & G & G & G & G & rs 8264884 & $A / G$ \\
\hline 02131.240779 & & & & G & A & G & A & G & A & A & A & A & A & rs 13476774 & $A / G$ \\
\hline 02131.352413 & & & & A & G & A & A & $A$ & G & G & G & G & G & rs 3690254 & $A / G$ \\
\hline 02131.372744 & Adra1d intron1 & agrees & I & A & G & $A$ & G & A & G & G & G & G & G & rs6206689 & $A / G$ \\
\hline 02131.487026 & & & & A & G & $A$ & G & A & G & G & G & G & G & rs 27288211 & $\mathrm{C} / \mathrm{T}$ \\
\hline 02131.679416 & & & & A & G & A & G & A & G & G & G & G & G & rs 4223510 & $\mathrm{C} / \mathrm{T}$ \\
\hline 02131.819271 & Rassf2 UTR & ... exon11,UTR & U & A & G & $A$ & G & A & G & G & G & G & G & rs 4223511 & $\mathrm{C} / \mathrm{T}$ \\
\hline 02132.094113 & Cds2 intron1 & & I & G & G & G & G & G & G & G & G & G & G & rs 3701463 & $\mathrm{C} / \mathrm{T}$ \\
\hline 02132.181539 & & & & A & $A$ & $A$ & A & A & A & $A$ & A & A & A & rs 3699051 & $A / G$ \\
\hline 02132.268510 & & 4921508D12Rik intron1 & & C & C & $\mathrm{C}$ & C & C & C & C & C & C & C & rs13476777 & $\mathrm{A} / \mathrm{C}$ \\
\hline 02132.423204 & & & & A & $A$ & A & A & $A$ & A & $A$ & $A$ & A & A & rs 13476778 & $\mathrm{C} / \mathrm{T}$ \\
\hline 02132.588030 & & & & G & G & G & G & G & G & G & G & G & G & rs 3722210 & $A / G$ \\
\hline 02132.631319 & 3300001M20Rik exon11 & Trmt6 exon11 & Trmt6 Cs L260 & A & $A$ & $A$ & $A$ & $A$ & A & $A$ & $A$ & A & A & rs6288723 & $\mathrm{C} / \mathrm{T}$ \\
\hline 02132.770061 & 5830467P10Rik intron1 & agrees & I & A & $A$ & $A$ & A & $A$ & A & $A$ & A & A & A & rs 13476779 & $A / G$ \\
\hline 02132.944863 & & & & G & G & G & G & G & G & G & G & G & G & rs 27240387 & $\mathrm{C} / \mathrm{T}$ \\
\hline
\end{tabular}


Table C-2. (Continued).

\begin{tabular}{|c|c|c|c|c|c|c|c|c|c|c|c|c|c|c|}
\hline $\begin{array}{l}\text { Position } \\
\text { (Chr Mb) } \\
\end{array}$ & NCBI gene annotation & Ensembl 48 gene annotation & $\begin{array}{l}\text { dbSNP } 128 \text { SNP } \\
\text { annotation }\end{array}$ & 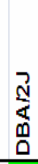 & 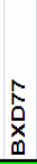 & 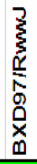 & 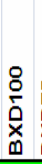 & & 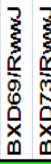 & 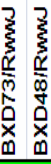 & 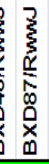 & 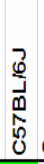 & $\mathrm{dbSNP}$ & Observed \\
\hline 02133.346268 & & & & $G$ & $G$ & $G$ & $\bar{G}$ & $\bar{G}$ & $\overline{G G}$ & $\overline{G G}$ & $G$ & G & rs 3676388 & $A / G$ \\
\hline 02133.388963 & & & Bmp2 L & A & A & A & A & A & A A & A A & $A$ & A & rs3022934 & $\mathrm{C} / \mathrm{T}$ \\
\hline 02133.535082 & & & & G & G & G & G & G & G G & $G \quad G$ & $G$ & G & rs3684247 & $A / G$ \\
\hline 02133.686867 & & & & G & G & G & G & G & G G & G $G$ & $G$ & G & rs 13476783 & $\mathrm{C} / \mathrm{T}$ \\
\hline 02133.748173 & & & & A & A & A & A & A & A A & A A & $A$ & $A$ & rs6397401 & AT \\
\hline 02134.133774 & & & & A & A & A & A & A & A A & $A$ A & A & A & rs6234650 & $A / G$ \\
\hline 02134.345047 & Hao1 intron4 & agrees & I & A & C & A & C & A & $\mathrm{C} \mathrm{C}$ & $\mathrm{C} \mathrm{C}$ & $C$ & C & rs13476784 & $\mathrm{AlC}$ \\
\hline 02134.586818 & & & & G & A & G & A & G & A A & $A$ A & $A$ & A & rs 13476785 & $\mathrm{CT}$ \\
\hline 02134.712881 & Plcb1 intron2 & agrees & 1 & C & $A$ & C & A & C & A A & $A A$ & A & A & rs 13476786 & $\mathrm{AlC}$ \\
\hline 02134.965497 & Plcb1 intron3 & agrees & 1 & A & G & A & $\mathrm{G}$ & A & G G & $G G$ & G & G & rs13476787 & $\mathrm{C} / \mathrm{T}$ \\
\hline 02135.137988 & Plcb1 intron11 & ... intron8 & I & G & A & G & A & G & A A & A A & $A$ & $A$ & rs 13476788 & $\mathrm{CT}$ \\
\hline 02135.360002 & & & & G & $A$ & G & A & G & A A & A A & $A$ & A & rs13476789 & $\mathrm{CT}$ \\
\hline 02135.469719 & & & & A & C & A & C & A & C C & C C & C & C & rs 13476790 & $\mathrm{AlC}$ \\
\hline 02135.592555 & Plcb4 intron1 & & I & G & $A$ & G & A & G & A A & $A$ A & $A$ & $A$ & rs6345820 & $A / G$ \\
\hline 02135.758395 & Plcb4 intron8 & ... intron7 & 1 & C & G & C & G & C & G G & $G \quad G$ & $G$ & G & rs29716787 & $C / G$ \\
\hline 02135.953692 & Pak7 intron3 & ... intron4 & 1 & A & G & A & G & A & $G G$ & $G G$ & $G$ & G & rs 27237140 & $\mathrm{CT}$ \\
\hline 02136.253017 & & & & C & $A$ & C & A & C & A A & A A & A & A & rs13476793 & $\mathrm{G} / \mathrm{T}$ \\
\hline 02136.567700 & Snap25 intron1 & agrees & 1 & A & $A$ & A & A & A & $A$ A & $A$ A & $A$ & $A$ & rs13476794 & $\mathrm{G} / \mathrm{T}$ \\
\hline 02136.704189 & Mkks intron3 & agrees & 1 & G & G & G & G & G & $G G$ & $G \quad G$ & $G$ & G & rs8246404 & $A / G$ \\
\hline 02137.050704 & & & & G & G & $G$ & G & G & $G G$ & $G \quad G$ & $G$ & G & rs3710324 & $\mathrm{C} / \mathrm{T}$ \\
\hline 02137.281348 & & & & A & $A$ & A & A & A & A A & $A$ A & $A$ & A & rs13476797 & $\mathrm{CT}$ \\
\hline 02137.513613 & & & & A & $A$ & $A$ & A & A & $A$ A & $A A$ & A & A & rs 13476798 & $\mathrm{AlC}$ \\
\hline 02137.618159 & & & & A & $A$ & A & A & $A$ & A A & $A$ A & A & $A$ & rs6249968 & $A / G$ \\
\hline 02137.970245 & & & & A & $A$ & A & A & A & A A & A A & $A$ & A & rs 3653457 & $\mathrm{AlC}$ \\
\hline 02138.305756 & & & & G & A & G & A & G & A A & $A \quad G$ & $A$ & A & rs13476801 & $\mathrm{C} / \mathrm{T}$ \\
\hline 02138.596276 & & & & C & C & $\mathrm{C}$ & C & C & $\mathrm{CC}$ & $\mathrm{CC}$ & $C$ & C & rs13476802 & $\mathrm{AlC}$ \\
\hline 02138.749442 & & & & A & A & A & A & A & A A & A A & A & A & rs6362243 & $\mathrm{CT}$ \\
\hline 02138.895273 & & & & A & $A$ & $A$ & A & A & A A & $A A$ & $A$ & A & rs6200333 & $A / G$ \\
\hline 02139.005406 & & & & C & C & C & C & C & $\mathrm{CO}$ & $C \mathrm{C}$ & $C$ & C & rs6158085 & $\mathrm{A} / \mathrm{C}$ \\
\hline 02139.322068 & C130053K05Rik intron2 & Sptc3 intron2 & Sptlc3l & C & G & C & G & C & $G G$ & $G C$ & $G$ & G & rs13476804 & $\mathrm{C} / \mathrm{G}$ \\
\hline 02139.549796 & 5430433G21Rik intron1 & & 1 & G & A & G & A & G & A A & $A G$ & A & $A$ & rs13476805 & $\mathrm{CT}$ \\
\hline 02139.558446 & 5430433G21Rik intron2 & & I & G & $A$ & G & A & G & $A$ A & $A G$ & A & A & rs6282055 & $\mathrm{CT}$ \\
\hline 02139.745898 & Tasp1 intron9 & ... intron10 & 1 & A & G & A & G & A & $G G$ & $G A$ & G & G & rs6251185 & $A / G$ \\
\hline 02140.191732 & Sel112 intron1 & agrees & 1 & G & A & G & A & G & A A & $A G$ & $A$ & $A$ & rs6238987 & $A / G$ \\
\hline 02140.632270 & & & & G & A & G & A & G & A A & $A G$ & $A$ & A & rs13476807 & $A / G$ \\
\hline 02140.949623 & & & & A & G & A & G & A & $G G$ & $G A$ & G & G & rs6186553 & $\mathrm{C} / \mathrm{T}$ \\
\hline 02140.949863 & & & & C & A & C & A & C & A A & $A C$ & A & A & rs6187777 & $\mathrm{G} / \mathrm{T}$ \\
\hline 02141.269051 & & & & G & A & G & A & G & A A & $A G$ & A & A & rs6303304 & $\mathrm{CT}$ \\
\hline 02141.506452 & & Macrod2 intron6 & & C & $A$ & C & A & C & A A & $A C$ & A & A & rs6346451 & $\mathrm{G} T$ \\
\hline 02141.778076 & & Macrod2 intron8 & & G & A & G & A & G & A A & $A \quad G$ & A & A & rs6397890 & $\mathrm{CT}$ \\
\hline 02142.006271 & & Macrod2 intron2 & & G & A & G & A & G & A A & $A G$ & A & A & rs6360457 & $A / G$ \\
\hline 02142.226462 & & & & A & G & A & G & A & $G G$ & $G A$ & G & G & rs 13476810 & $A / G$ \\
\hline 02142.529801 & Kif16b intron20 & ... intron21 & 1 & A & G & A & G & $A$ & $G G$ & G A & G & G & rs6331493 & $\mathrm{CT}$ \\
\hline 02142.540462 & Kif16b exon18 & agrees & Cs E596 & A & G & A & G & A & $G G$ & $G A$ & G & G & rs13476811 & $\mathrm{CT}$ \\
\hline 02142.725202 & Kif16b intron1 & agrees & 1 & C & G & C & G & C & $G G$ & $G C$ & G & G & Is 13476812 & $\mathrm{C} / \mathrm{G}$ \\
\hline 02142.911592 & & & & A & C & A & C & A & $\mathrm{CO}$ & C A & C & C & rs13476813 & $\mathrm{G} / \mathrm{T}$ \\
\hline 02143.215177 & & & & G & A & G & A & G & $A$ A & $A G$ & A & A & rs13476814 & $\mathrm{CT}$ \\
\hline 02143.420472 & Pcsk2 intron2 & agrees & 1 & G & A & G & A & G & A A & $A G$ & $A$ & $A$ & rs6195594 & $A / G$ \\
\hline 02143.748895 & Dstn intron1 & agrees & 1 & A & G & A & $G$ & A & $G G$ & $G A$ & G & G & rs 3687229 & $\mathrm{CT}$ \\
\hline 02143.770431 & & & & G & A & G & A & G & A A & $A \quad G$ & $A$ & A & rs33511869 & $\mathrm{CT}$ \\
\hline 02143.786839 & Rrbp1 intron11 & ... intron10 & 1 & C & A & C & A & C & A A & $A C$ & $A$ & A & rs3707946 & $\mathrm{AlC}$ \\
\hline 02144.199389 & Csrp2bp exon2 & agrees & Cs T18 & A & G & A & G & $A$ & $G G$ & $G A$ & G & G & rs13476817 & $A / G$ \\
\hline 02144.359114 & Polr3f intron 4 & agrees & 1 & G & A & G & A & G & A A & $A G$ & $A$ & A & rs 3655895 & $A / G$ \\
\hline 02144.582696 & Dtd1 intron5 & agrees & 1 & G & A & G & A & G & A A & $A G$ & $A$ & A & rs 13476818 & $\mathrm{C} / \mathrm{T}$ \\
\hline 02144.594327 & Dtd1 UTR & agrees & U & A & G & A & G & A & $G G$ & $G A$ & G & G & rs3023694 & $A / G$ \\
\hline 02144.636566 & & & & G & A & G & A & G & A A & $A G$ & A & A & rs 3661718 & $A / G$ \\
\hline
\end{tabular}


Table C-2. (Continued).

\begin{tabular}{|c|c|c|c|c|c|c|c|c|c|c|c|c|c|c|}
\hline $\begin{array}{l}\text { Position } \\
\text { (Chr Mb) }\end{array}$ & NCBI gene annotation & Ensembl 48 gene annotation & $\begin{array}{l}\text { dbSNP } 128 \text { SNP } \\
\text { annotation }\end{array}$ & 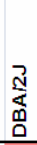 & $\begin{array}{l}\hat{N} \\
\substack{\times \\
0} \\
\end{array}$ & 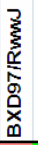 & $\begin{array}{l}\stackrel{8}{\circ} \\
\substack{x \\
0} \\
\end{array}$ & 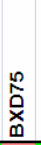 & 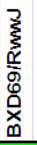 & 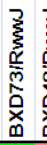 & 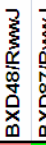 & 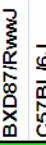 & 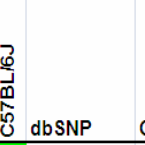 & Observed \\
\hline 02144.810573 & & & & A & G & A & $\mathrm{G}$ & A & G & $G A$ & $A G$ & $G \quad G$ & $\begin{array}{ll}\text { G } & \text { rs6170159 }\end{array}$ & $\mathrm{C} \pi$ \\
\hline 02145.107384 & Slc24a3 intron2 & agrees & 1 & G & A & G & A & G & A & A C & G A & A A & A rs3022909 A & $A / G$ \\
\hline 02145.433591 & Slc24a3 intron6 & ... intron12 & 1 & G & A & G & A & G & A & A C & G A & A A & A rs3679762 A & A/G \\
\hline 02145.712336 & Rin2 UTR & ... exon13,UTR & U & A & A & A & A & A & A & A A & A A & A A & A rs27272367 A & A/G \\
\hline 02145.858075 & & 4930529M08Rik intron14 & & A & A & A & A & A & A & A A & A A & A A & A rs6363071 A & $A / G$ \\
\hline 02145.996022 & & & & A & A & A & A & A & A & $A A$ & A A & A A & A rs 13476820 & $\mathrm{C} / \mathrm{T}$ \\
\hline 02146.117744 & & A230067G21 Rik intron38 & & A & A & A & A & A & A & A A & A A & A A & A rs3676491 C & $\mathrm{G} / \mathrm{T}$ \\
\hline 02146.464837 & & & & A & A & A & A & A & A & A A & A A & A A & A rs 3668423 A & $A / G$ \\
\hline 02146.524475 & & & & A & A & A & A & A & A & A A & A A & A A & A rs6254019 A & $A / G$ \\
\hline 02146.880833 & Xrn2 intron24 & agrees & 1 & A & A & A & A & A & A & $A A$ & A A & A A & A $\quad$ rs 3724835 & $A / G$ \\
\hline 02147.204386 & & & & A & A & A & A & $A$ & A & $A A$ & A A & A A & A rs13476822 A & $A / G$ \\
\hline 02147.254465 & & & & A & A & A & A & A & A & $A A$ & A A & A A & A rs3701696 & $\mathrm{C} / \mathrm{T}$ \\
\hline 02147.477700 & LOC640914 intron1 & & & A & G & A & G & A & G & $G A$ & $A G$ & G G & G rs 3696870 & $A / G$ \\
\hline 02147.607368 & & & & $\mathrm{~T}$ & $T$ & T & T & $T$ & T & T $T$ & T $\mathrm{T}$ & T T & T rs 3683143 & ATT \\
\hline 02147.753900 & & & & G & A & G & A & G & A & $A C$ & G A & A A & A rs 13476825 & $\mathrm{C} / \mathrm{T}$ \\
\hline 02147.881059 & & & & G & A & G & A & A & A & A C & G A & A A & A rs6209325 C & $\mathrm{C} / \mathrm{T}$ \\
\hline 02147.932881 & & & & A & G & A & G & G & G & $G A$ & $A G$ & G G & G rs6332517 & $\mathrm{C} / \mathrm{T}$ \\
\hline 02148.222019 & Sstr4 exon1 & agrees & Cs P270 & C & A & C & A & A & A & A C & C A & A A & A rs29556174 & $\mathrm{G} / \mathrm{T}$ \\
\hline 02148.230161 & & & Thbd L & A & G & A & G & G & G & $G A$ & $A G$ & $G \quad G$ & G rs4223558 & $A / G$ \\
\hline 02148.230256 & Thbd UTR & ... exon1,UTR & U & C & C & C & C & C & C & $\mathrm{CO}$ & $\mathrm{C} \mathrm{C}$ & $\mathrm{C} C$ & C rs4223557 A & $\mathrm{A} / \mathrm{C}$ \\
\hline 02148.232342 & Thbd exon1 & agrees & Cs G446 & A & G & A & G & G & G & $G A$ & $A G$ & G G & G rs13476826 & $A / G$ \\
\hline 02148.357423 & & & & G & A & G & A & A & A & A C & G A & A A & A Is13476827 & $A / G$ \\
\hline 02148.573862 & & & & c & G & c & G & G & G & G C & C G & $G \quad G$ & G rs6288803 & $\mathrm{C} / \mathrm{G}$ \\
\hline 02148.697668 & Cst3 UTR & ...exon3,UTR & U & A & G & A & G & G & G & $G A$ & $A G$ & G G & G $\quad$ rs8256617 & $A / G$ \\
\hline 02148.975537 & & & & A & G & A & G & G & G & G A & $A G$ & G G & G Is13476830 A & $A / G$ \\
\hline 02149.070623 & & & & G & A & G & A & A & A & A C & G A & A A & A rs3723883 & $\mathrm{C} / \mathrm{T}$ \\
\hline 02149.271015 & & & & $T$ & A & T & A & A & A & A T & T A & A A & A rs 3676033 & AT \\
\hline 02149.491613 & & & & A & G & A & G & G & G & $G A$ & $A G$ & $G \quad G$ & G rs6206791 A & $A / G$ \\
\hline 02149.561210 & EG667048 intron1 & & & C & A & C & A & $A$ & A & $A C$ & C A & A A & A rs13476832 & $\mathrm{A} / \mathrm{C}$ \\
\hline 02149.688083 & & & OTTMUSG00000015750 I & A & A & A & A & A & A & A A & A A & A A & A rs6246342 A & $A / G$ \\
\hline 02149.750008 & OTMUSG00000015750 intron1 & & 1 & A & A & A & A & A & A & A A & A A & A A & A rs6176485 A & $A / G$ \\
\hline 02149.804749 & OTTMUSG00000015750 intron2 & & 1 & A & G & A & G & G & G & $G A$ & A G & G G & G $\quad$ rs $13476833 A$ & $\mathrm{~A} / \mathrm{G}$ \\
\hline
\end{tabular}


Table C-3. SNP analysis of genes in mapped locus on Chr X between 50-100 Mb.

\begin{tabular}{|c|c|c|c|c|c|c|c|c|c|c|c|c|c|c|c|}
\hline $\begin{array}{l}\text { Position } \\
\text { (Chr Mb) } \\
\end{array}$ & NCBI gene annotation & $\begin{array}{l}\text { Ensembl } 48 \text { gene } \\
\text { annotation }\end{array}$ & $\begin{array}{l}\text { dbSNP } 128 \\
\text { SNP } \\
\text { annotation }\end{array}$ & 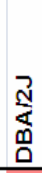 & $\begin{array}{l}\hat{N} \\
\stackrel{x}{x} \\
0\end{array}$ & 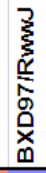 & 옴 & 㔯 & 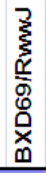 & 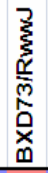 & 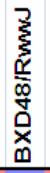 & 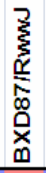 & 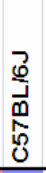 & dbSNP & Observed \\
\hline X50.304196 & Phf6 intron8 & ... intron11 & 1 & $A$ & A & $\mathrm{C}$ & $\mathrm{C}$ & $\bar{C}$ & $\mathrm{C}$ & $A$ & $\mathrm{C}$ & $\bar{A}$ & $\mathrm{C}$ & rs13483756 & $\mathrm{G} / \mathrm{T}$ \\
\hline$X 50.441456$ & Plac1 intron2 & agrees & 1 & A & A & G & G & G & G & A & G & A & G & rs13483757 & $\mathrm{C} / \mathrm{T}$ \\
\hline$X 50.688138$ & & & & A & A & G & G & G & G & A & G & A & $\mathrm{G}$ & rs13483758 & $\mathrm{C} / \mathrm{T}$ \\
\hline$\times 51.078618$ & Zfp36l3 exon1 & agrees & $\mathrm{Cn} \mathrm{M} 268 \mathrm{~V}$ & G & G & A & A & A & A & G & A & G & A & rs13483759 & $\mathrm{C} / \mathrm{T}$ \\
\hline$X 53.469071$ & & & & A & A & $A$ & A & $A$ & $A$ & A & $A$ & A & A & rs13483761 & $A / G$ \\
\hline$X 53.755235$ & Ddx26b intron14 & agrees & 1 & A & A & $A$ & A & $A$ & A & A & A & A & A & rs6202026 & $\mathrm{A} / \mathrm{G}$ \\
\hline X 54.012901 & Fhl1 intron3 & ... intron2 & 1 & A & A & $A$ & A & $A$ & A & A & A & A & A & rs13483763 & $\mathrm{C} / \mathrm{T}$ \\
\hline X 54.787689 & & & & A & A & $A$ & C & $\mathrm{C}$ & C & A & C & A & C & rs13483765 & $\mathrm{A} / \mathrm{C}$ \\
\hline X 55.021542 & & & & A & $A$ & $A$ & G & G & G & A & G & A & G & rs13483766 & $\mathrm{C} / \mathrm{T}$ \\
\hline X 55.119516 & & & & A & $A$ & $A$ & $\mathrm{~T}$ & $\mathrm{~T}$ & $\mathrm{~T}$ & A & $\mathrm{T}$ & A & $\mathrm{T}$ & rs13483767 & ATT \\
\hline X 55.423039 & & & & G & G & A & A & A & A & G & A & G & A & rs13483768 & $\mathrm{C} / \mathrm{T}$ \\
\hline$X 55.907643$ & & & & A & A & $\mathrm{T}$ & $T$ & $\mathrm{~T}$ & $T$ & A & $T$ & A & $\mathrm{T}$ & rs13483769 & AT \\
\hline X 56.198835 & & & & A & A & G & G & G & G & A & G & A & G & rs29057816 & $\mathrm{C} / \mathrm{T}$ \\
\hline X 56.322051 & Fgf13 intron3 & agrees & I & G & G & $G$ & $G$ & G & $G$ & G & G & G & G & rs6361318 & $\mathrm{C} / \mathrm{T}$ \\
\hline$X 56.488675$ & & & & G & G & A & G & A & A & G & A & G & A & rs13483770 & $A / G$ \\
\hline$X 56.638971$ & & & & A & A & $A$ & A & $A$ & A & A & $A$ & A & A & rs13483771 & $\mathrm{A} / \mathrm{G}$ \\
\hline$X 56.786516$ & & & & G & G & G & G & G & G & G & G & G & G & rs31736534 & $\mathrm{C} / \mathrm{T}$ \\
\hline$X 57.174959$ & & & & G & G & $G$ & G & G & G & G & G & G & G & rs6172548 & $A / G$ \\
\hline$X 58.428522$ & & C230004F18Rik exon7 & & G & G & G & G & G & G & G & G & G & G & rs13483777 & $\mathrm{C} / \mathrm{T}$ \\
\hline$X 58.726530$ & & & & A & $A$ & $A$ & A & A & A & A & $A$ & A & A & rs13483778 & $\mathrm{A} / \mathrm{G}$ \\
\hline$\times 59.012359$ & & & & A & A & $A$ & A & $A$ & A & $A$ & $A$ & A & A & rs6156066 & $\mathrm{A} / \mathrm{G}$ \\
\hline$\times 59.629009$ & & & & A & A & $A$ & A & $A$ & A & A & $A$ & $A$ & $A$ & rs13483781 & $\mathrm{A} / \mathrm{G}$ \\
\hline X 59.725144 & & & & A & A & $A$ & A & A & A & A & $A$ & $A$ & A & rs6315050 & $\mathrm{A} / \mathrm{G}$ \\
\hline X 60.353111 & & & & G & G & $G$ & G & G & G & G & G & G & G & rs6273297 & $A / G$ \\
\hline X 60.678266 & & & & G & G & $G$ & G & G & G & G & G & G & G & rs6371771 & $\mathrm{C} / \mathrm{T}$ \\
\hline X 60.897610 & & & & A & A & $A$ & A & $A$ & A & A & $A$ & A & A & rs13483783 & $\mathrm{C} / \mathrm{T}$ \\
\hline X 61.595306 & & & & A & A & G & A & G & G & A & G & A & G & rs13483785 & $\mathrm{A} / \mathrm{G}$ \\
\hline X 61.868817 & & & & C & $\mathrm{C}$ & A & C & A & A & C & A & C & A & rs13483786 & $\mathrm{A} / \mathrm{C}$ \\
\hline$X 62.557333$ & & & & $\mathrm{~T}$ & $\mathrm{~T}$ & $\mathrm{~T}$ & $T$ & $\mathrm{~T}$ & $T$ & $\mathrm{~T}$ & $T$ & $\mathrm{~T}$ & $\mathrm{~T}$ & rs13483790 & $\mathrm{A} T \mathrm{~T}$ \\
\hline X 62.802036 & & & & G & G & G & G & G & G & G & G & G & G & rs 3695410 & $A / G$ \\
\hline X 62.871894 & & & & A & A & $A$ & A & A & A & A & A & A & A & rs13483791 & $\mathrm{G} / \mathrm{T}$ \\
\hline X 63.211829 & & & & C & $\mathrm{C}$ & $\mathrm{C}$ & C & $\mathrm{C}$ & $\mathrm{C}$ & C & C & C & C & rs6317266 & $\mathrm{A} / \mathrm{C}$ \\
\hline X 63.548433 & & & & A & A & $A$ & A & A & A & A & A & A & A & rs13483793 & AT \\
\hline X63.963690 & & & & G & G & $G$ & G & $G$ & G & G & G & G & G & rs31231784 & $A / G$ \\
\hline X 64.133911 & & & & G & G & G & G & G & G & $G$ & G & G & G & rs29048726 & $\mathrm{C} / \mathrm{T}$ \\
\hline X 64.489187 & & & & A & A & $A$ & A & A & A & A & A & A & $A$ & rs29049502 & $\mathrm{G} / \mathrm{T}$ \\
\hline X 64.565854 & & & & G & G & $G$ & G & G & G & G & G & G & G & rs31212020 & $\mathrm{C} / \mathrm{T}$ \\
\hline$X 64.717310$ & & & & A & A & $A$ & A & A & A & A & $A$ & A & A & rs33870777 & $\mathrm{C} / \mathrm{T}$ \\
\hline X 64.860968 & & & & G & G & $G$ & G & $G$ & G & $G$ & G & G & G & rs31306210 & $\mathrm{C} / \mathrm{T}$ \\
\hline$\times 65.033446$ & & & & C & $\mathrm{C}$ & $\mathrm{C}$ & $\mathrm{C}$ & $\mathrm{C}$ & $\mathrm{C}$ & $\mathrm{C}$ & $\mathrm{C}$ & $\mathrm{C}$ & C & rs29048228 & $\mathrm{A} / \mathrm{C}$ \\
\hline X 65.202453 & & & & A & A & $A$ & A & $A$ & A & A & A & A & $A$ & rs29051320 & $\mathrm{G} / \mathrm{T}$ \\
\hline$\times 65.262708$ & & & & G & G & $G$ & G & G & G & $G$ & G & G & G & rs6296283 & $\mathrm{C} / \mathrm{T}$ \\
\hline$\times 65.406453$ & & & & G & G & $G$ & $G$ & $G$ & G & G & G & G & G & rs13483797 & $\mathrm{A} / \mathrm{G}$ \\
\hline X 65.868220 & & & & G & G & $G$ & $G$ & $\mathrm{G}$ & G & $G$ & G & G & G & rs29049652 & $\mathrm{A} / \mathrm{G}$ \\
\hline$X 66.262536$ & & & & C & $\mathrm{C}$ & $\mathrm{C}$ & C & C & C & $\mathrm{C}$ & C & C & C & rs13483808 & $\mathrm{G} / \mathrm{T}$ \\
\hline$\times 66.607415$ & & & & A & $A$ & C & A & C & C & A & C & A & C & rs13483810 & $\mathrm{G} / \mathrm{T}$ \\
\hline X 66.798088 & Aff2 exon3 & agrees & $\mathrm{Cn}$ T228A & G & G & A & G & A & A & G & A & G & A & rs13483811 & $\mathrm{A} / \mathrm{G}$ \\
\hline X 66.915814 & & Aff2 intron3 & I & G & $G$ & $A$ & G & A & A & G & A & G & A & rs3089604 & $\mathrm{C} / \mathrm{T}$ \\
\hline X 67.347285 & & & & C & $\mathrm{C}$ & $A$ & C & A & A & C & A & C & A & rs13483813 & $\mathrm{A} / \mathrm{C}$ \\
\hline X 68.020243 & & & & A & A & G & A & G & G & A & G & A & G & rs13483814 & $\mathrm{A} / \mathrm{G}$ \\
\hline X 68.130578 & & & & G & G & $A$ & G & A & A & G & A & G & A & rs13483815 & $\mathrm{A} / \mathrm{G}$ \\
\hline X 68.621588 & Mtmr1 intron1 & agrees & I & A & $A$ & A & A & A & A & A & A & $A$ & A & rs6159220 & AT \\
\hline X 68.694215 & Cd99l2 intron3 & ... intron4 & I & G & G & $A$ & A & $A$ & A & G & A & G & A & rs3157124 & $\mathrm{C} / \mathrm{T}$ \\
\hline X 69.187248 & & & & A & $A$ & G & G & G & G & $A$ & G & A & G & rs13483818 & $\mathrm{A} / \mathrm{G}$ \\
\hline X 69.924084 & & & & G & G & $\mathrm{C}$ & $\mathrm{C}$ & $\mathrm{C}$ & $\mathrm{C}$ & $G$ & C & G & C & rs13483821 & $\mathrm{C} / \mathrm{G}$ \\
\hline$\times 70.261115$ & Zfp185 exon11 & agrees & Cn I211V & G & G & A & A & A & A & G & A & G & A & rs13483822 & $A / G$ \\
\hline
\end{tabular}


Table C-3. (Continued).

\begin{tabular}{|c|c|c|c|c|c|c|c|c|c|c|c|c|c|c|c|}
\hline $\begin{array}{l}\text { Position } \\
\text { (Chr Mb) }\end{array}$ & NCBI gene annotation & $\begin{array}{l}\text { Ensembl } 48 \text { gene } \\
\text { annotation }\end{array}$ & $\begin{array}{l}\text { dbSNP } 128 \\
\text { SNP } \\
\text { annotation }\end{array}$ & 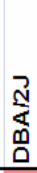 & $\begin{array}{l}\hat{s} \\
\stackrel{x}{x} \\
0\end{array}$ & 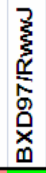 & $\underset{\substack{O \\
\hdashline}}{\stackrel{0}{x}}$ & $\begin{array}{l}\stackrel{n}{0} \\
\stackrel{x}{x} \\
0\end{array}$ & 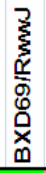 & 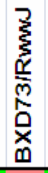 & 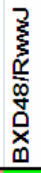 & 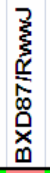 & 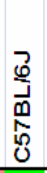 & dbSNP & Observed \\
\hline$\times 70.591777$ & Zfp275 intron2 & agrees & 1 & A & A & G & G & G & $\mathrm{G}$ & A & G & A & G & rs13483823 & $\mathrm{C} / \mathrm{T}$ \\
\hline X70.862405 & & & & G & G & G & G & G & G & G & G & G & G & rs6291099 & $\mathrm{C} / \mathrm{T}$ \\
\hline X 71.121919 & L1cam intron1 & agrees & 1 & A & A & G & G & G & A & A & G & A & G & rs13483824 & $A / G$ \\
\hline X71.452881 & Tktl1 intron12 & agrees & I & A & A & G & G & G & A & A & G & A & G & rs13483825 & $A / G$ \\
\hline X71.578525 & Plxna3 intron4 & agrees & 1 & G & G & A & A & A & G & G & A & G & A & rs29036935 & $A / G$ \\
\hline X 71.702281 & & & & A & A & $A$ & A & $A$ & $A$ & A & $A$ & A & A & rs 13483826 & $\mathrm{C} / \mathrm{T}$ \\
\hline X 72.973693 & & & & A & A & $A$ & A & $A$ & $A$ & A & A & A & A & rs13483831 & $A / G$ \\
\hline X 73.568953 & & & & A & A & G & G & G & A & A & G & A & G & rs13483834 & $\mathrm{C} / \mathrm{T}$ \\
\hline X73.669338 & & & & C & C & $A$ & A & $A$ & C & C & A & C & A & rs 29032402 & $\mathrm{~A} / \mathrm{C}$ \\
\hline X73.867519 & & & & G & G & A & A & $A$ & G & G & A & G & A & rs 29034080 & $A / G$ \\
\hline X 73.994756 & & & & C & $C$ & $A$ & A & $A$ & C & C & A & C & A & rs29033159 & $A / C$ \\
\hline X 74.333567 & & & & G & G & $\mathrm{C}$ & C & C & G & G & C & G & C & rs 29031390 & $\mathrm{C} / \mathrm{G}$ \\
\hline X 74.437611 & & & & A & A & G & G & G & A & A & $G$ & A & G & rs13483838 & $A / G$ \\
\hline$\times 76.270169$ & & & & A & A & $A$ & A & $A$ & $A$ & A & A & A & A & rs6232687 & $A / G$ \\
\hline X 76.642086 & LOC100041373 intron13 & & 1 & G & G & G & G & G & G & G & G & G & G & rs 6400558 & $\mathrm{C} / \mathrm{T}$ \\
\hline X 76.792464 & & & & G & G & A & A & A & G & G & A & G & A & rs29033641 & $\mathrm{C} / \mathrm{T}$ \\
\hline$\times 76.974502$ & & & & C & C & $\mathrm{C}$ & C & C & C & $\mathrm{C}$ & C & C & C & rs6358828 & GT \\
\hline X 77.128597 & & & & A & $A$ & G & G & G & A & A & G & A & G & rs31114399 & $\mathrm{C} / \mathrm{T}$ \\
\hline$X 77.442176$ & & & & C & C & A & A & A & C & $\mathrm{C}$ & A & C & A & rs13483849 & $\mathrm{G} / \mathrm{T}$ \\
\hline X 77.596981 & & & & $\mathrm{~T}$ & $T$ & A & A & A & $\mathrm{T}$ & $\mathrm{T}$ & A & $\mathrm{T}$ & A & rs 29030057 & ATT \\
\hline$\times 77.758255$ & & & & A & A & G & G & G & A & A & G & A & G & rs 33872327 & $\mathrm{C} / \mathrm{T}$ \\
\hline X77.964147 & & & & C & C & G & G & G & C & C & G & C & G & rs13483852 & $\mathrm{C} / \mathrm{G}$ \\
\hline X 78.316989 & Tmem47 intron1 & agrees & I & A & A & G & G & G & A & $A$ & G & A & G & rs13483853 & $A / G$ \\
\hline X78.387471 & & & & A & A & C & C & C & A & A & C & A & C & rs29122940 & $\mathrm{G} / \mathrm{T}$ \\
\hline X 78.565195 & & & & C & $\mathrm{C}$ & A & A & $A$ & C & $\mathrm{C}$ & A & C & A & rs29122347 & $\mathrm{A} / \mathrm{C}$ \\
\hline X78.716893 & & & & G & G & C & $\mathrm{C}$ & C & G & G & C & G & C & rs29124734 & $\mathrm{C} / \mathrm{G}$ \\
\hline X 79.175607 & & & & A & A & C & C & C & A & A & C & A & C & rs13483857 & $\mathrm{G} / \mathrm{T}$ \\
\hline X 79.446294 & & & & G & G & $A$ & A & $A$ & G & G & A & G & A & rs13483858 & $\mathrm{C} / \mathrm{T}$ \\
\hline X 79.763566 & & & & G & G & $A$ & A & $A$ & G & G & A & G & A & rs29121137 & $A / G$ \\
\hline X 79.962339 & & & & A & $A$ & G & G & G & A & A & G & A & G & rs29120700 & $A / G$ \\
\hline X 80.269841 & Dmd intron1 & agrees & I & A & A & G & G & G & A & A & G & A & G & rs13483862 & $A / G$ \\
\hline$X 80.517265$ & Dmd intron2 & agrees & 1 & A & A & G & G & G & A & A & G & A & G & rs13483863 & $\mathrm{C} / \mathrm{T}$ \\
\hline X 80.674835 & & & Dmd I & G & G & A & A & A & G & G & A & G & A & rs31396732 & $\mathrm{C} / \mathrm{T}$ \\
\hline X 80.819498 & Dmd intron1 & ... intron7 & 1 & A & A & $\mathrm{T}$ & $\mathrm{T}$ & $\mathrm{T}$ & A & A & $\mathrm{T}$ & A & $\mathrm{T}$ & rs 29063388 & ATT \\
\hline$X 81.021583$ & Dmd intron13 & ... intron19 & 1 & G & G & G & G & G & G & G & G & G & G & rs6389792 & $\mathrm{C} / \mathrm{G}$ \\
\hline X 81.087944 & Dmd intron23 & ... intron29 & 1 & G & G & $A$ & $A$ & $A$ & G & G & A & G & A & rs13483864 & $\mathrm{C} / \mathrm{T}$ \\
\hline$X 81.485465$ & Dmd intron38 & ... intron44 & I & $\mathrm{C}$ & $\mathrm{C}$ & $\mathrm{C}$ & $\mathrm{C}$ & $C$ & $\mathrm{C}$ & $\mathrm{C}$ & C & C & C & rs6276382 & $\mathrm{G} / \mathrm{T}$ \\
\hline X81.842456 & Dmd intron6 & ... intron53 & 1 & A & $A$ & G & G & $G$ & A & $A$ & G & A & G & rs13483868 & $A / G$ \\
\hline X 82.043294 & Dmd intron 10 & ... intron57 & I & A & A & G & G & G & A & A & G & A & G & rs13483869 & $A / G$ \\
\hline X 82.133393 & Dmd intron13 & ... intron60 & 1 & C & C & A & A & $A$ & C & $\mathrm{C}$ & A & C & A & rs3675552 & $A / C$ \\
\hline X 82.563944 & & & & G & $G$ & $A$ & $A$ & A & G & G & A & G & A & rs13483870 & $\mathrm{C} / \mathrm{T}$ \\
\hline X 82.743905 & & & & G & $G$ & $A$ & $A$ & $A$ & G & G & A & G & A & rs3725966 & $\mathrm{C} / \mathrm{T}$ \\
\hline X83.044868 & & & & G & G & C & $\mathrm{C}$ & $\mathrm{C}$ & G & G & C & G & C & rs31117642 & $\mathrm{C} / \mathrm{G}$ \\
\hline X 83.314902 & & & & A & A & G & G & G & A & A & $G$ & A & G & rs13483873 & $A / G$ \\
\hline X 83.474831 & & & & G & $G$ & $A$ & A & A & G & G & $A$ & G & A & rs 33867225 & $\mathrm{C} / \mathrm{T}$ \\
\hline X 83.632157 & & & & A & A & G & G & G & A & A & G & A & G & rs31319847 & $A / G$ \\
\hline X 83.754274 & & & & G & G & $A$ & A & $A$ & G & G & A & G & A & rs3696812 & $A / G$ \\
\hline X 84.193868 & \|1rapl1 intron5 & ... intron6 & 1 & G & G & A & $A$ & A & G & G & A & G & A & rs13483877 & $A / G$ \\
\hline X 84.490778 & \|1 rapl1 intron4 & ... intron5 & 1 & $\mathrm{~T}$ & $T$ & A & $A$ & A & $\mathrm{T}$ & $\mathrm{T}$ & A & $\mathrm{T}$ & A & rs13483878 & AIT \\
\hline X84.635263 & \|1 rapl1 intron2 & ... intron3 & 1 & A & A & $A$ & $A$ & $A$ & $A$ & A & A & A & A & rs6380311 & $\mathrm{G} / \mathrm{T}$ \\
\hline X84.917154 & & Il1rapl1 intron2 & & A & $A$ & $A$ & $A$ & A & $A$ & A & $A$ & $A$ & A & rs6228331 & $A / G$ \\
\hline$X 85.166458$ & & |l1rapl1 intron1 & & G & $G$ & G & G & $G$ & $G$ & G & $G$ & G & G & rs6179920 & $A / G$ \\
\hline$\times 85.700670$ & & & & G & G & G & G & G & G & G & G & G & G & rs6212120 & $\mathrm{C} / \mathrm{T}$ \\
\hline X86.920572 & & & & A & A & G & G & G & A & A & G & A & G & rs 13483803 & $\mathrm{C} / \mathrm{T}$ \\
\hline$X 87.440160$ & & & & G & G & A & $A$ & A & G & G & A & G & A & rs 13483805 & $A / G$ \\
\hline X 87.724390 & & & & A & A & A & A & A & A & A & A & A & A & rs6211131 & $\mathrm{C} / \mathrm{T}$ \\
\hline
\end{tabular}


Table C-3. (Continued).

\begin{tabular}{|c|c|c|c|c|c|c|c|c|c|c|c|c|c|c|c|}
\hline $\begin{array}{l}\text { Position } \\
\text { (Chr Mb) } \\
\end{array}$ & NCBI gene annotation & $\begin{array}{l}\text { Ensembl } 48 \text { gene } \\
\text { annotation }\end{array}$ & $\begin{array}{l}\text { dbSNP } 128 \\
\text { SNP } \\
\text { annotation }\end{array}$ & 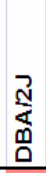 & $\stackrel{\hat{N}}{\stackrel{x}{x}}$ & 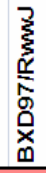 & 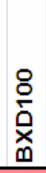 & $\stackrel{n}{\stackrel{n}{0}}$ & 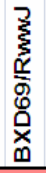 & 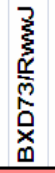 & 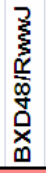 & 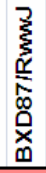 & 它 & $\mathrm{dbSNP}$ & Observed \\
\hline X87.943903 & & & & A & A & A & A & A & A & A & A & A & A & rs6221067 & $\mathrm{A} / \mathrm{G}$ \\
\hline$X 88.357437$ & & & & A & A & A & A & A & A & A & A & A & A & rs6259276 & AVT \\
\hline X 88.466052 & & & & G & G & G & G & G & G & G & G & G & G & rs6162389 & $\mathrm{C} / \mathrm{T}$ \\
\hline$X 89.326263$ & EG236892 intron1 & & I & G & G & G & G & G & G & G & G & G & G & rs6224816 & $A / G$ \\
\hline$X 89.566020$ & & & & G & G & G & G & G & G & G & G & G & G & (no-rs) & $? / ?$ \\
\hline X89.920839 & & & & C & C & A & A & A & C & $\mathrm{C}$ & A & C & A & rs13483880 & $\mathrm{G} / \mathrm{T}$ \\
\hline$\times 90.058794$ & & & & C & C & G & G & G & C & C & G & C & G & rs29070461 & $\mathrm{C} / \mathrm{G}$ \\
\hline X90.183658 & & & & A & A & C & C & C & A & A & C & A & C & rs 29072783 & $\mathrm{~A} / \mathrm{C}$ \\
\hline$\times 90.523800$ & & & & A & A & G & G & G & A & A & G & A & G & rs13483881 & $A / G$ \\
\hline$\times 91.150210$ & EG668103 intron1 & & I & A & A & $A$ & A & A & A & A & A & A & A & rs13483884 & $\mathrm{C} / \mathrm{T}$ \\
\hline X91.583744 & & & LOC631145 L & A & A & $A$ & A & A & A & A & A & A & A & rs13483885 & $\mathrm{C} / \mathrm{T}$ \\
\hline X91.781474 & Maged1 exon12 & agrees & Cs A686 & G & G & G & G & G & G & G & G & G & G & rs13459181 & $A / G$ \\
\hline X92.246917 & Arhgef9 UTR & ... intron10 & U & A & A & $A$ & A & A & A & A & A & A & A & rs13483887 & $A / G$ \\
\hline X92.516668 & & & & G & G & G & G & G & G & G & G & G & G & rs13483888 & $\mathrm{C} / \mathrm{T}$ \\
\hline$\times 92.842774$ & AK129302 intron1 & agrees & I & G & G & G & G & G & G & G & G & G & G & rs13483890 & $\mathrm{C} / \mathrm{T}$ \\
\hline$\times 93.098708$ & A130028J20Rik intron4 & Zc3h12b intron5 & Zc3h12b I & A & A & $A$ & A & A & A & A & A & A & A & rs13483891 & $\mathrm{A} / \mathrm{C}$ \\
\hline$\times 93.378516$ & & & & G & G & G & G & G & G & G & G & G & G & rs13483892 & $A / G$ \\
\hline X93.694393 & Heph exon12 & agrees & Cn N592S & A & A & A & A & A & A & A & A & A & A & rs13483894 & $A / G$ \\
\hline X94.171734 & & & & $\mathrm{T}$ & $\mathrm{T}$ & $\mathrm{T}$ & $\mathrm{T}$ & $\mathrm{T}$ & $\mathrm{T}$ & $\mathrm{T}$ & $\mathrm{T}$ & $\mathrm{T}$ & $\mathrm{T}$ & rs6182892 & AVT \\
\hline X94.404878 & & & & G & G & G & G & G & G & G & G & G & G & rs6309772 & $\mathrm{C} / \mathrm{T}$ \\
\hline$\times 94.532103$ & Eda2r UTR & agrees & U & A & A & $A$ & A & A & A & A & A & A & A & rs6367841 & $\mathrm{G} / \mathrm{T}$ \\
\hline X95.119353 & & & & A & A & A & A & $A$ & $A$ & A & A & A & $A$ & rs13483898 & $A / G$ \\
\hline$\times 95.472455$ & $\mathrm{Ar}$ intron2 & agrees & I & A & A & $A$ & A & A & A & A & A & A & A & rs 3672400 & $\mathrm{C} / \mathrm{T}$ \\
\hline X 95.543918 & & & & G & G & G & G & G & G & G & G & G & G & rs29086868 & $\mathrm{C} / \mathrm{G}$ \\
\hline$\times 95.727753$ & & & & A & A & $A$ & A & $A$ & A & A & A & A & $A$ & rs13483899 & $\mathrm{A} / \mathrm{C}$ \\
\hline X95.921452 & Ophn1 exon7 & agrees & Cs A162 & A & A & A & A & A & A & A & A & A & A & rs 13483900 & $\mathrm{C} / \mathrm{T}$ \\
\hline X96.090321 & & & & A & A & A & A & A & A & A & $A$ & A & $A$ & rs 3680829 & AVT \\
\hline X96.259400 & Stard8 intron3 & agrees & 1 & A & A & A & A & A & A & A & A & A & A & rs13483902 & $\mathrm{C} / \mathrm{T}$ \\
\hline X96.844849 & & & & A & A & $A$ & A & $A$ & A & A & A & A & A & rs29081061 & $\mathrm{C} / \mathrm{T}$ \\
\hline X97.014720 & & & EG620592 L & G & G & $G$ & G & G & $G$ & G & G & G & G & rs31142709 & $A / G$ \\
\hline X97.191531 & Eda intron1 & agrees & I & G & G & $G$ & G & G & G & G & G & G & $G$ & rs 29083820 & $A / G$ \\
\hline X97.614860 & & & & G & G & $G$ & G & G & G & G & G & G & G & rs13483909 & $A / G$ \\
\hline X97.921890 & Kif4 exon30 & agrees & Cs A1195 & G & G & G & G & G & G & G & G & G & G & rs 13483910 & $A / G$ \\
\hline X97.922028 & Kif4 UTR & ... exon30,UTR & U & C & C & $\mathrm{C}$ & C & C & $\mathrm{C}$ & $\mathrm{C}$ & C & C & $\mathrm{C}$ & rs4232594 & $\mathrm{C} / \mathrm{G}$ \\
\hline X97.961994 & & & $D \lg 3 \mathrm{~L}$ & A & A & A & A & A & A & A & A & A & A & rs3714964 & $\mathrm{C} / \mathrm{T}$ \\
\hline X98.027382 & & & & G & G & $G$ & G & G & G & G & G & G & G & rs13483911 & $\mathrm{C} / \mathrm{T}$ \\
\hline X98.333835 & & Snx12 intron4 & & C & C & C & C & $\mathrm{C}$ & $\mathrm{C}$ & C & C & C & $\mathrm{C}$ & rs29079390 & $\mathrm{G} / \mathrm{T}$ \\
\hline X98.847444 & Ogt intron3 & agrees & I & G & G & $G$ & G & G & G & G & G & G & G & rs6171419 & $\mathrm{C} / \mathrm{T}$ \\
\hline X99.620143 & Hdac8 intron7 & agrees & I & A & A & A & A & G & A & $\mathrm{H}$ & G & $\mathrm{H}$ & G & rs29080049 & $A / G$ \\
\hline X99.935066 & & & & $\mathrm{C}$ & $\mathrm{C}$ & $\mathrm{C}$ & $\mathrm{C}$ & $\mathrm{C}$ & $\mathrm{C}$ & $\mathrm{C}$ & $\mathrm{C}$ & $\mathrm{C}$ & $\mathrm{C}$ & rs31253166 & $\mathrm{G} / \mathrm{T}$ \\
\hline
\end{tabular}




\section{APPENDIX D. LIST OF PUBLISHED LOCI AND GENES ON CHROMOSOME 2 THAT ARE ASSOCIATED WITH DIFFERENTIAL SUSCEPTIBILITY TO INFECTIOUS DISEASES}

This appendix contains a list of quantitative trait loci (QTLs) and genes on $\mathrm{Chr} 2$ that were found by published studies investigating susceptibility to infectious diseases other than GAS sepsis, based on literature search on May 3, 2010. From the list below, only one study used recombinant inbred (RI) mice strains (CXB-RI strains) (Thach et al., 2001). Other retrieved studies used either F2 crosses or backcrossed of some strains of interest. 
Table D-1. Diseases and mouse models associated with quantitative trait loci and genes located at Chromosome 2 loci mapped in current study.

\begin{tabular}{|c|c|c|c|}
\hline Disease & Model and Strain Used & Candidate Genes/Loci & References \\
\hline $\begin{array}{l}\text { Neuroadapted Sindbis } \\
\text { virus (NSV) }\end{array}$ & $\begin{array}{l}\mathrm{C} 57 \mathrm{Bl} / 6 \mathrm{~J} \text { and } \mathrm{Balb} / \mathrm{cBy}, \mathrm{CXB} \text { inbred } \\
\text { mice }\end{array}$ & $\begin{array}{l}\text { QTL on Chr } 2 \text { near D2Mit } 447 \\
(60 \mathrm{cM})\end{array}$ & (Thach et al., 2001) \\
\hline Chlamydia trachomatis & $\begin{array}{l}\mathrm{C} 57 \mathrm{~B} 1 / 6 \mathrm{~J} \text { and } \mathrm{C} 3 \mathrm{H} / \mathrm{HeJ} \text { and } \mathrm{F} 2 \text { crosses } \\
\text { and congenic }\end{array}$ & QTLs on Chr 2, 3 and 11 & $\begin{array}{l}\text { (Bernstein-Hanley et } \\
\text { al., 2006) }\end{array}$ \\
\hline $\begin{array}{l}\text { Borrelia burgdorferi } \\
\text { (lyme disease) }\end{array}$ & $\begin{array}{l}\mathrm{C} 57 \mathrm{BL} / 6 \mathrm{~N} X \mathrm{C} 3 \mathrm{H} / \mathrm{HeN} \\
(\mathrm{C} 57 \mathrm{BL} / 6 \mathrm{~N} X \mathrm{C} 3 \mathrm{H} / \mathrm{HeN}) \mathrm{F} 1 \mathrm{X} \mathrm{C} 3 \mathrm{H} / \mathrm{HeN} \\
\text { and }(\mathrm{C} 57 \mathrm{BL} / 6 \mathrm{~N} \text { X C3H/HeN) F1 X } \\
\text { C57BL/6N backcrosses }\end{array}$ & $\begin{array}{l}\text { Bbaa15 locus and proximity of } \\
\text { markers D2Mit278 and D2Mit224 }\end{array}$ & (Roper et al., 2001) \\
\hline Leishmania major & $\mathrm{F} 2$ crosses of $(\mathrm{CcS}-16 \times \mathrm{BALB} / \mathrm{c})$ & Lmr14 & $\begin{array}{l}\text { (Havelkova et al., } \\
\text { 2006) (Zhang and } \\
\text { Matlashewski, 2004) } \\
\text { (Vladimirov et al., } \\
\text { 2003) }\end{array}$ \\
\hline Leishmania donovani & $\begin{array}{l}\text { B10.LP-H-3 b and B10.CE }(30 \mathrm{NX}) \text { and } \\
(\mathrm{B} 10 . \mathrm{LP}-\mathrm{H}-3 \mathrm{~b} \times \mathrm{B} 10) \mathrm{F} 1\end{array}$ & Immune response locus 2 (Ir2) & DeTolla 1980 \\
\hline Candida albicans. & {$[\mathrm{A} / \mathrm{J} \times \mathrm{C} 57 \mathrm{BL} / 6 \mathrm{~J}] \mathrm{F} 2$ crosses } & Hemolytic complement (Hc or C5) & $\begin{array}{l}\text { (Tuite } \text { et al., 2004; } \\
\text { Tuite et al., 2005) }\end{array}$ \\
\hline Salmonella typhimurium & (AcB61 x 129S6) F2 crosses & Hemolytic complement (Hc or C5) & (Roy et al., 2007) \\
\hline Listeria monocytogenes & $\mathrm{A} / \mathrm{J}$ and $\mathrm{B} 6$ & Hemolytic complement (Hc or C5) & $\begin{array}{l}\text { (Czuprynski et al., } \\
\text { 2003) }\end{array}$ \\
\hline
\end{tabular}




\section{VITA}

Nourtan Fatthy Abdeltawab, a Cairo, Egypt native, was born on June 14, 1979. She graduated from the English high school, Education Home School, Dokki, Giza, in 1997. She received her Bachelor's degree, Excellent with highest honors, in Pharmaceutical Sciences, College of Pharmacy, Cairo University 2002. She was then appointed as a teaching assistant at Department of Microbiology and Immunology, College of Pharmacy, Cairo University where she worked on studies towards her M.Sc. degree. In August 2004, Nourtan joined The University of Tennessee Health Science Center in Memphis, for her PhD studies. Summer of 2005, Nourtan joined the research team of Dr. Malak Kotb laboratory, where she worked on her dissertation project. In September 2008, Nourtan relocated with Dr. Kotb to University of Cincinnati, Ohio, where she finished her dissertation work. She is expected to graduate in May 2010 from University of Tennessee Health Science Center. 